\title{
Autobiographical memory retrieval : overgeneral memory and intrusions
}

Citation for published version (APA):

Hauer, B. J. A. (2008). Autobiographical memory retrieval : overgeneral memory and intrusions. [Doctoral Thesis, Maastricht University]. Datawyse / Universitaire Pers Maastricht. https://doi.org/10.26481/dis.20080222bh

Document status and date:

Published: 01/01/2008

DOI:

10.26481/dis.20080222bh

Document Version:

Publisher's PDF, also known as Version of record

\section{Please check the document version of this publication:}

- A submitted manuscript is the version of the article upon submission and before peer-review. There can be important differences between the submitted version and the official published version of record.

People interested in the research are advised to contact the author for the final version of the publication, or visit the DOI to the publisher's website.

- The final author version and the galley proof are versions of the publication after peer review.

- The final published version features the final layout of the paper including the volume, issue and page numbers.

Link to publication

\footnotetext{
General rights rights.

- You may freely distribute the URL identifying the publication in the public portal. please follow below link for the End User Agreement:

www.umlib.nl/taverne-license

Take down policy

If you believe that this document breaches copyright please contact us at:

repository@maastrichtuniversity.nl

providing details and we will investigate your claim.
}

Copyright and moral rights for the publications made accessible in the public portal are retained by the authors and/or other copyright owners and it is a condition of accessing publications that users recognise and abide by the legal requirements associated with these

- Users may download and print one copy of any publication from the public portal for the purpose of private study or research.

- You may not further distribute the material or use it for any profit-making activity or commercial gain

If the publication is distributed under the terms of Article $25 \mathrm{fa}$ of the Dutch Copyright Act, indicated by the "Taverne" license above, 


\section{Autobiographical memory retrieval}

Overgeneral memory and intrusions 
(c) Beatrijs Hauer, Amsterdam 2008

All rights reserved. No part of this book may be translated or reproduced in any form by photo, photoprint, microfilm or any other means without written permission from the author.

ISBN 978-90-5278-697-1

Cover illustration:

View over Maastricht from Havenstraat 42D

Design and lay-out: Beatrijs \& Nils

Publisher: Datawyse, Universitaire Pers Maastricht 


\section{Autobiographical memory retrieval}

\section{Overgeneral memory and intrusions}

Proefschrift ter verkrijging van de graad van doctor aan de Universiteit van Maastricht, op gezag van de Rector Magnificus Prof. Mr. G.P.M.F. Mols volgens het besluit van het College van Decanen, in het openbaar te verdedigen op vrijdag 22 februari 2008 om 14:00 uur, door Beatrijs Jacoba Aleida Hauer geboren op 28 maart 1980, te Utrecht.

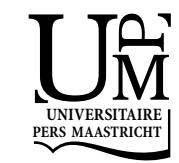


Promotor

Prof. dr. H.L.G.J. Merckelbach

\section{Copromotores}

Dr. I. Wessel

Dr. T. Dalgleish (MRC Cognition and Brain Sciences Unit, Cambridge, United Kingdom)

Beoordelingscommissie

Prof. dr. C. de Ruijter (voorzitter)

Dr. I.M. Engelhard (Universiteit Utrecht)

Prof. dr. D. Hermans (Katholieke Universiteit Leuven)

Dr. F. Peeters

Prof. dr. M. Peters 


\section{Contents}

1 Overgeneral memory in depression and posttraumatic stress disorder: Co-occurrence with intrusions

2 Intrusions, avoidance and overgeneral memory in a non-clinical sample

3 Retrieval-induced forgetting of autobiographical memory details

4 Effects of repeated retrieval of central and peripheral details in complex emotional slides

5 Effect of suppressing negative memories on intrusions and autobiographical memory specificity in a non-clinical sample

6 Autobiographical memory specificity after manipulating retrieval cues in adults reporting childhood sexual abuse

7 Prepartum autobiographical memory specificity predicts posttraumatic stress symptoms following complicated pregnancy

8 General discussion and conclusion

9 Samenvatting

References

Dankwoord / Acknowledgements

Curriculum Vitae

Publications 



\title{
Overgeneral memory in depression and posttraumatic stress disorder: Co-occurrence with intrusions
}

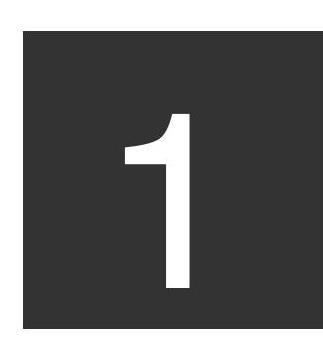

\author{
B.J.A. Hauer, I. Wessel, H. Merckelbach
}

Extended and adapted from: Tijdschrift voor Psychiatrie (2005: 47, 299-308)*

* Overalgemene herinneringen bij depressie en bij de posttraumatische stress stoornis: De rol van intrusieve herinneringen 
Imagine the following situation: You are thinking back of that day you spent in Venice a couple of years ago. You remember walking on the San Marco square covered with doves at sunrise. You re-experience the smell of a delicious espresso and your happy feeling of being there by yourself without any other tourists. Thinking back, you once more conclude that this was an amazing experience.

This is a typical autobiographical memory that contains episodic details and self-relevant information. Moreover, autobiographical memory is an aspect of episodic memory (Tulving, 2002), which is the type of memory that allows people to reflect upon personal experiences from the past. In fact, it makes people able to mentally travel through time. Retrieval of such a memory is, therefore, more than an objective description (i.e., semantic memory, 'When is your birthday?' and 'Where do you live?'). The person retrieving the memory, consciously experiences the fact that he is remembering a past event that is included with personal perspectives, self-awareness, emotions, thoughts, and perceptual details such as visual images. Hence, episodic and self-relevant information are relevant components of autobiographical memory.

Autobiographical memory has been studied in different contexts. For example, research has focused on the underlying processes of how personal events are encoded, retrieved and forgotten (e.g., Hertel \& Gerstle, 2003; Tulving, 2002). Other research has particularly investigated the nature and function of autobiographical memory (e.g., Pillemer, 1998; Rubin, 1996) or its relation with early development (e.g., Nelson \& Fivush, 2004) and with the concept of self (e.g., Conway \& Pleydell-Pearce, 2002; Nelson \& Fivush, 2004). The work presented in the present thesis will be contextualized in terms of Conway et al.'s model about autobiographical memory. This is the Self Memory System (SMS) in which autobiographical memory is explained in relation to the concept of "the self" and its goals (Conway, 2005; Conway \& Pleydell-Pearce, 2000; Conway, Singer, \& Tagini, 2004). 


\section{Autobiographical memory}

\section{Organization}

According to the Self Memory System (e.g., Conway et al., 2004), autobiographical memory consists of four essential components. The first of these is the autobiographical memory knowledge base. In a hierarchical structure, it contains life story schemas, lifetime periods, and general events. The life story schemas consist of knowledge about ones global personal history like 'my career as a scientist'. At the second level, the lifetime periods contain periods of information that reflect overarching goals and activities, such as 'living in Maastricht' or 'writing my PhD thesis'. These lifetime periods can be temporally and thematically organized. Furthermore, some of these periods may overlap, like 'part of the time that I was living in Maastricht, I also wrote my PhD thesis'. At the bottom level, general events are categories of more specific experiences about a shared theme, like 'going to conferences' and 'first-time experiences', or a certain period of time such as 'my vacation in Italy'.

The second component is the conceptual self that consists of abstract knowledge about the self. In contrast to the autobiographical knowledge base, it contains non-temporal self structures like self-images and self-guides (i.e., 'Who am I?' 'Who do I want to be?') as well as attitudes, values, and beliefs (i.e., 'What beliefs do I have, and why?' 'What is important to me?'). It helps to define the self, other people, and general interactions with others and the world. The conceptual self and the autobiographical memory knowledge base form the basis of autobiographical memory and together they are called the long-term self. However, in order to retrieve a specific autobiographical memory with the accompanying recollective experience, like that morning at San Marco square in Venice, a third component is needed. This is the episodic memory system (also termed as episodic memories or event specific knowledge) that includes sensory and perceptual details, such as visual images, sound and smell. Taken together, knowledge from the autobiographical memory knowledge base, conceptual self and the episodic memory system are fundamental components of an autobiographical memory (see Figure 1.1 for a schematic illustration of these components). 


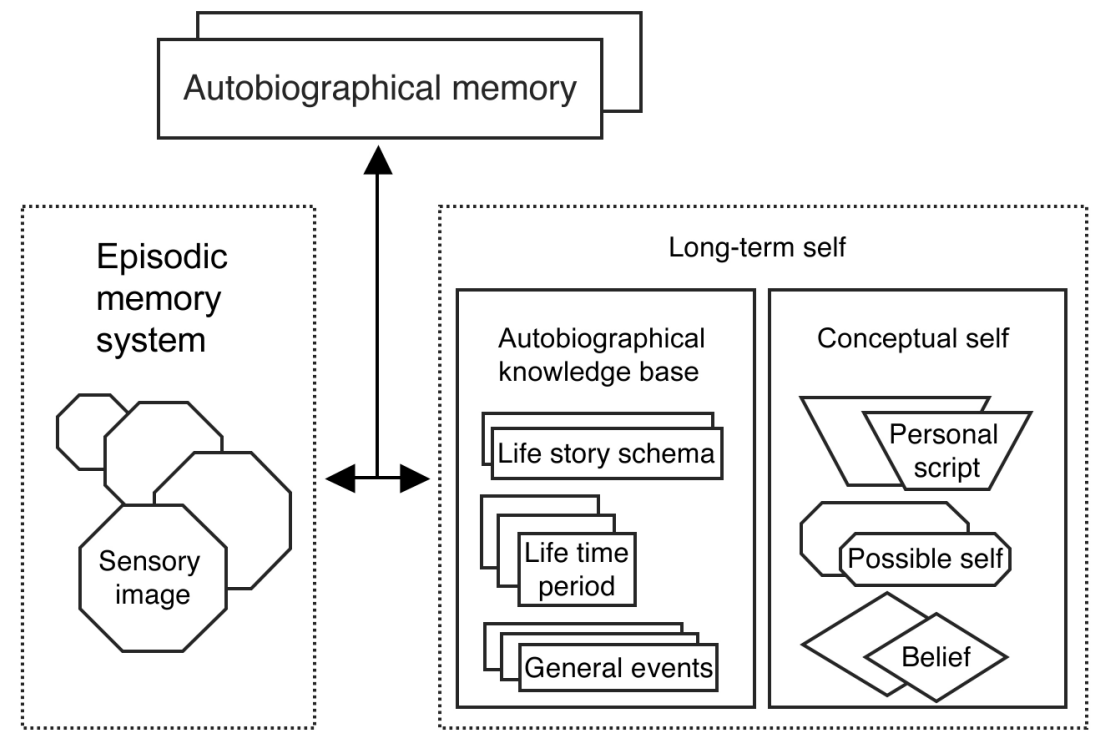

Figure 1.1. Generation of autobiographical memories (Conway et al., 2004, p.498).

In order to bind the above components into an actual autobiographical memory, one additional component is necessary. This is the working self (see figure 1.2). It links the autobiographical knowledge base, conceptual self, and episodic memories together by means of current goals. The working self is concerned with the present and continuously organizes it by setting goals and priorities. In fact, it regulates short-term goals, such as cooking dinner, and is concerned with processes of what should be done to attain these goals. In these situations, relevant memories are formed in function of attaining such short-term goals (e.g., getting a pan, cutting the vegetables). Here, the most important function of memories is that they correspond with goals that need to be attained. It may, therefore, not be surprising that many memories only remain in consciousness as long as they have a function, for example as long as you are preparing your dinner. Once their function has gone, memories disappear from the stream of consciousness. Moreover, a lot of information may not even be stored because it does not match with current goals. 


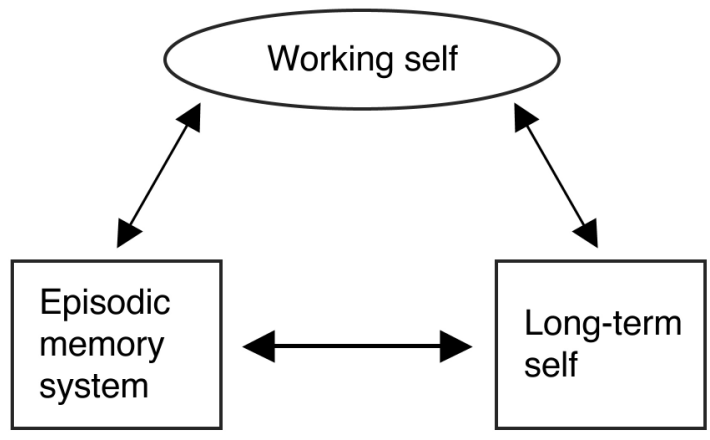

Figure 1.2. The Self-Memory System (Conway et al., 2004, p.499).

Memories can also be integrated with knowledge to become stored in the autobiographical memory knowledge base. This usually happens when they contribute to a stable and coherent record of the self about who you are and how you look upon the world. Here, goals are long-term and relevant to the selfconcept. Coherence of a memory with your concept of self is important in "deciding" whether the memory will be stored. If an experience is incongruent with the self-concept or difficult to fit in, the experience may not be stored or it might take much more effort to integrate it into the autobiographical memory knowledge structures.

To summarize, autobiographical memories contain information from the autobiographical memory knowledge base, self-concept, and episodic memory system. Once these components are linked together by the working self, a complete autobiographical memory is represented that gives you the recollective experience from the morning at San Marco square in Venice. Which memories are formed or retrieved highly depends on current and desired goals and the priority that is given to them by the working self. 


\section{Retrieval processes}

Imagine the following situation: It is a sunny morning and you are pouring an espresso for yourself. At once you have this elaborate memory of that day in Venice at the San Marco square. It was the smell of espresso together with the sunny morning and the pleasant temperature that out of the blue made you reexperience this past event. This type of remembering fundamentally differs from the next example. You are sitting in the sun together with your friend and you talk about vacations that you have spent together. Your friend is curious about your most beautiful vacation-memory. You have been to France, Berlin and Italy. Yes, Italy. You made a trip to Verona, Vicenza and also stayed in Venice for a couple of days. Venice was lovely, going to Murano, walking around in the narrow streets and visiting the San Marco cathedral. Yes, that morning at the square with the smell of coffee and without any other people was one of the best days of the vacation.

Basically, there are two ways in which we retrieve memories (Conway, 2005; Conway \& Pleydell-Pearce, 2000; Conway et al., 2004). Both retrieval modes can lead, though not necessarily, to the same autobiographical memory. In the first example, one speaks of direct retrieval. This is an associative process in which a(n episodic) retrieval cue (e.g., smell of coffee, sunny morning) directly maps onto a specific episodic memory which then comes to consciousness. This is a bottom-up process that is practically automatic and does not need much attention and retrieval effort. It is a spontaneous recollection. In terms of the above-mentioned working self and active goals, direct memory retrieval may or may not be related to active goals. Subsequently, the working self may 'decide' whether this popped-up memory is worth following with (new) active goals or not.

The second example is a matter of generative retrieval. This is an active topdown search process that requires effort. Here one actively and deliberately tries to find the right retrieval cues to remember the most beautiful vacationmemory. Usually, this generative search process starts at the level of life time periods or general events. Together with the correct retrieval cue, a specific episodic memory is retrieved. This process of generative retrieval usually matches the active goals that are set by the working self, which, therefore, 
generally leads to memories that both match with the generative search criteria as well as with the active goals.

\section{Autobiographical memory and psychopathology}

Autobiographical memory is a central aspect of human functioning and contributes to an individual's sense of the self (e.g., Conway et al., 2004). It also helps people to get a better sense of the world, to pursue goals in problem solving situations, and to regulate emotions. For these reasons, it may not be surprising that autobiographical memory plays a role in psychopathology. Indeed, research has demonstrated that various forms of psychopathology, specifically emotional disorders like major depression and posttraumatic stress disorder (PTSD), are characterized by autobiographical memory retrieval patterns that systematically differ from those of healthy individuals (e.g., Conway \& Pleydell-Pearce, 2000). For example, individuals exposed to a psychological trauma describe frequent intrusive and distressing recollections of the traumatic event (see McNally, 2003) and depressed individuals report a preponderance of negatively-valenced memories of their past (e.g., Beck, Rush, Shaw, \& Emery, 1979).

The influence of mood on memory has indeed been studied thoroughly (see for example Williams, Watts, MacLeod, \& Mathews, 1997). One important finding of this research is that when people are in a negative mood, they will more easily encode and retrieve words that similarly have a negative valence. This phenomenon has been termed mood-congruent memory. In an illustrative study, Williams and Broadbent (1986) hypothesized that such a moodcongruent effect would also occur within autobiographical memory. They investigated this by measuring the speed of recall of autobiographical memories in suicidal patients and hospitalized control patients (only having physical symptoms). All of the patients were asked to retrieve specific autobiographical memories according to positive (e.g., happy, interested) and negative (e.g., regret, angry) cue words as fast as possible. Williams and Broadbent (1986) defined a specific memory as a memory of a single event that happened at a particular place that lasted less than one day.

Their results indeed demonstrated the mood-congruency effect: relative to control patients, suicidal patients reported negative memories much faster than 
positive memories. To examine the antecedents of this effect, Williams and Broadbent explored the content of the memories. They discovered that memories of suicidal patients were much more general than those of controls. This was particularly true for memories retrieved on the basis of positive cue words. In response to the cue word 'happy', participants with physical problems usually recalled a specific memory, such as 'when I had dinner with my friends in Maastricht last Saturday'. Suicidal patients, however, tended to come up with phrases like, 'when I was young' or 'when I am at work'. Thus, despite the explicit instruction to retrieve specific memories, suicidal patients were inclined to report general descriptions of several events. Williams and Broadbent termed this phenomenon overgeneral memory. This more or less serendipitous finding has resulted in a blooming research line (see for a review Williams et al., 2007).

\section{Overgeneral memory}

The term specific autobiographical memory refers to a memory of a personally experienced event that happened at a particular place and lasted less than a day. Failing to retrieve specific autobiographical memories is called overgeneral memory. Typically, two types of overgeneral memory are distinguished (Williams \& Dritschel, 1991). On the one hand, extended memories refer to a single event that lasted longer than one day, for example 'when I was at high school'. On the other hand, categoric memories refer to a group of similar events that occurred on repeated occasions, such as 'every time I go to my parents'. Several studies have demonstrated that especially the frequency of specific and categoric memories is related in a predictable way to certain forms of psychopathology (e.g., Williams et al., 2007).

Since Williams and Broadbent (1986) introduced a cueing methodology, many studies have used a similar method to measure overgeneral memory. This cueing task, the Autobiographical Memory Test (AMT), involves the presentation of cue words varying in emotional valence. Typically, an equal number of positive and negative words are presented to participants (see for a meta-analysis van Vreeswijk \& de Wilde, 2004). Individuals are told that the event recalled can be important or trivial, recent or a long time ago, but that it should be a specific autobiographical experience that happened at a particular time and place, and lasted less than a day. Subsequent to some practice trials, 
the actual task is administered and usually participants are given 30 or 60 s to respond to each cue word with a specific autobiographical memory (van Vreeswijk \& de Wilde, 2004). In terms of Conway et al.'s model, this is a generative retrieval task. Participants are intentionally searching for a memory that meets the search criteria. Thus, in this context, autobiographical memory retrieval is a generative process.

After Williams and Broadbent's (1986) initial study, later research has shown that overgeneral memory is not restricted to suicidal patients. Various studies have demonstrated that individuals with a major depression have an overgeneral memory (e.g., Hermans et al., 2004; Wessel, Meeren, Peeters, Arntz, \& Merckelbach, 2001; Williams \& Scott, 1988). Reduced memory specificity has also been found in PTSD (e.g., McNally, Lasko, Macklin, \& Pitman, 1995; McNally, Litz, Prassas, Shin, \& Weathers, 1994), acute stress disorder (ASD; Harvey, Bryant, \& Dang, 1998; Kangas, Henry, \& Bryant, 2005), eating disorder (Dalgleish et al., 2003), obsessive compulsive disorder (OCD; Wilhelm, McNally, Baer, \& Florin, 1997), borderline personality disorder (BPD; Jones et al., 1999; Kremers, Spinhoven, \& van der Does, 2004), and schizophrenia (Kaney, Bowen-Jones, \& Bentall, 1999; Riutort, Cuervo, Danion, Peretti, \& Salame, 2003), though not in other anxiety disorders than ASD and PTSD (Wessel et al., 2001). Interestingly, comorbid depression often explained the presence of overgeneral memory in samples with other clinical diagnoses (e.g., Kremers et al., 2004; Wilhelm et al., 1997). Thus, the relative difficulty with retrieving specific autobiographical memories is particularly associated with emotional disorders such as major depression and trauma-related psychopathology (e.g., PTSD).

\section{Clinical relevance}

Why is it important that people are able to retrieve specific autobiographical memories? As stated above, autobiographical memory retrieval is particularly important to pursue goals in problem solving situations and to regulate emotions (e.g., Conway et al., 2004). Consequently, overgeneral memory tends to interfere with normal human functioning in everyday life. In particular, individuals with an overgeneral memory retrieval style have trouble with problem solving, 
recover less well from therapy and seem vulnerable for psychopathology (e.g., Williams et al., 2007).

\section{Problem solving capacity}

Several studies have demonstrated that overgeneral memory is related to poor social problem solving skills (e.g., Evans, Williams, OLoughlin, \& Howells, 1992; Goddard, Dritschel, \& Burton, 1996; 1997; Raes et al., 2005). For example, Goddard et al. (1996; 1997) revealed that individuals with and without major depression who displayed an overgeneral memory were often unable to verbalize what they would do to make new friends in an unfamiliar environment. Thus, finding it difficult to remember specific memories about similar past experiences may hinder successful interpersonal contact and interaction with the environment. Related to this, overgeneral memory hampers the ability to make specific simulations of the future (Williams, Ellis, Tyers, MacLeod, \& Rose, 1996). For example, when asking participants to make concrete plans for the future, Williams et al. (1996) found that participants who were less specific in their memory retrieval were often unable to crystallize their prospective plans. Taken together, specific memory retrieval helps people to solve day-to-day problems and to direct their life toward specific aims that they would like to achieve.

\section{Prognosis}

Overgeneral memory also influences the course of depression (e.g., Brittlebank, Scott, Williams, \& Ferrier, 1993; Dalgleish, Spinks, Yiend, \& Kuyken, 2001; Peeters, Wessel, Merckelbach, \& Boon-Vermeeren, 2002). Brittlebank et al. (1993), for example, reported that reduced autobiographical memory specificity at the start of treatment predicted delayed recovery in individuals with major depression, over and above initial levels of depressive symptoms. Dalgleish et al. (2001) found similar effects in a sample of individuals with seasonal affective disorder. They showed that overgeneral memory retrieval to positive cue words in winter was related to more depressive symptoms in summer. However, in a sample of depressed individuals, Raes and his colleagues demonstrated that 
when they controlled for patients' rumination ${ }^{1}$ overgeneral memory was no longer predictive for higher levels of depression seven months later (Raes et al., 2006). Thus, overgeneral memory seems to be relevant in the course of depression. Nevertheless, its predictive role may be mediated by third variables such as rumination.

Besides this role of overgeneral memory during the course of depression, several studies have demonstrated that patients who had recovered from depression were still less specific in their autobiographical memory recall than never depressed individuals (e.g., Mackinger, Pachinger, Leibetseder, \& Fartacek, 2000; Williams \& Dritschel, 1988). This suggests that overgeneral memory is a trait-like phenomenon. However, other studies found that overgeneral memory did not significantly predict treatment outcome (Brewin, Reynolds, \& Tata, 1999; Spinhoven et al., 2007). Furthermore, other studies found that after specific training, depressed patients were able to retrieve more specific memories than they could before (Watkins \& Taesdale, 2001; Williams, Teasdale, Segal, \& Soulsby, 2000). Taken together, this leaves us with mixed results on this matter up to now. Overgeneral memory seems a relevant factor in treatment outcome. However, some studies did not find such an effect. And after specific training, depressed individuals seem to be able to retrieve more specific autobiographical memories again.

\section{Vulnerability}

Prospective studies with non-clinical individuals further tell us that memory specificity may also be a vulnerability factor to develop depressive symptoms after a stressful experience. To begin with, Mackinger, Loschin, and Leibetseder (2000) found that overgeneral memory to negative cues administered during pregnancy was related to more depressive symptoms three months after giving birth. Thus, young mothers who retrieved more overgeneral memories during their pregnancy suffered from more postpartum depressive symptoms. Additionally, van Minnen, Wessel, Verhaak, and Smeenk (2005) showed that

\footnotetext{
${ }^{1}$ Ruminations are abstract representations about depressive symptoms, negative emotions, and the possible implications of these (e.g., '...I have always failed at university' and '...I will never graduate'; Nolen-Hoeksema, 2000; Watkins, Teasdale, \& Williams, 2000).
} 
women who retrieved fewer specific memories before the start of an in vitro fertilization treatment demonstrated more depressive and anxiety symptoms after treatment failure (i.e., no pregnancy). In another study, Gibbs and Rude (2004) revealed that college students with increased levels of overgeneral memory at baseline and who had frequently experienced stressful events in the subsequent 4-6 weeks also reported heightened levels of depressive symptoms at the end of this period. Overall, these data suggest that reduced memory specificity before a stressful experience may predict later depressive symptoms.

To summarize, overgeneral autobiographical memory is related to reduced social problem solving skills and to being less able to formulate concrete plans about the future. Furthermore, although the results are mixed and third variables may play a role, overgeneral memory tends to be related to a less beneficial course of depression. It also appears to be a vulnerability factor to develop depressive symptoms after a stressful experience.

\section{Overgeneral memory and trauma}

Up to now, the focus has been primarily on overgeneral memory in depression. However, the above prospective studies (e.g., van Minnen et al., 2005) indicated that overgeneral memory before a life-stress experience predicts more depressive symptoms (e.g., diathesis-stress perspective, Scher, Ingram, \& Segal, 2005). Furthermore, major depression is a common outcome following traumatic experiences (e.g., Shalev et al., 1998). What is more, non-clinical samples with a trauma history (e.g., Raes, Hermans, Williams, \& Eelen, 2005), traumatized individuals with PTSD (e.g., McNally et al., 1994; 1995; Schönfeld \& Ehlers, 2006), and ASD (e.g., Harvey et al., 1998; Kangas et al., 2005) display overgeneral memory retrieval. Taken together, these findings point toward a potentially important role of traumatic experiences in overgeneral memory retrieval. Indeed, there is empirical evidence that a traumatic background is related to lack of autobiographical memory specificity (e.g., Williams et al., 2007). 


\section{Empirical evidence}

Kuyken and Brewin (1995) were the first to find that depressed women with a history of childhood sexual abuse (CSA) retrieve more overgeneral memories than depressed women who have never been abused. These findings suggest that trauma might strengthen reduced memory specificity. Since this initial study, similar results have been found in other samples of depressed women reporting CSA (Burnside, Startup, Byatt, Rollinson, \& Hill, 2004) and in nonclinically depressed women with a history of CSA (Henderson, Hargreaves, Gregory, \& Williams, 2002). Overgeneral memory has also been established in samples with different types of trauma such as burn injury (e.g., Stokes, Dritschel, \& Bekerian, 2004), motor vehicle accidents (Harvey et al., 1998), war trauma (e.g., McNally et al., 1995; Wessel, Merckelbach, \& Dekkers, 2002), assault (Schönfeld, Ehlers, Böllinghaus, \& Rief, 2007), childhood trauma (e.g., de Decker, Hermans, Raes, \& Eelen, 2003; Hermans et al., 2004) as well as in non-clinical samples with various self-reported traumatic experiences (e.g., Raes et al., 2005; though see Wessel et al., 2001; Willebrand et al., 2002). Overall, the bottom line of these studies is that traumatized individuals depressed or non-depressed - retrieve fewer specific autobiographical memories than non-traumatized control groups and this is true for both clinical and non-clinical samples.

There are, to our knowledge, only two longitudinal studies that have examined the role of overgeneral memory in the course of PTSD. In the first study, Harvey et al. (1998) investigated whether memory specificity would predict PTSD six months later in individuals with acute stress disorder (ASD) related to a motor vehicle accident. Recall of fewer specific memories about trauma-related events predicted PTSD at follow-up six months later. There was no such association between trauma-unrelated memories and later PTSD. However, using a similar design in a sample of cancer patients with ASD, Kangas et al. (2005) were not able to replicate this because in their sample memory specificity did not predict PTSD six months later. These mixed findings might be due to two rather different trauma samples (see also Moore \& Zoellner, 2007). It was clear that the motor vehicle accident survivors were assessed after having experienced their trauma. In contrast, the cancer patients were potentially experiencing ongoing medical procedures, and thus they may still have been in the middle of 
their trauma experience at time of assessment. Hence, additional longitudinal and prospective studies are recommended to shed more light on this matter.

Taken together, various studies reported a relationship between trauma experiences and overgeneral memory (see Williams et al., 2007 for a review). Moreover, there are indications that reduced memory specificity not only predicts the course of depression (e.g., Brittlebank et al., 1999) but also of PTSD (Harvey et al., 1998). However, not all individuals who report having a history of trauma display an overgeneral autobiographical memory (e.g., Harvey et al., 1998; Kangas et al., 2005; MacNally et al., 1994; 1995). For example, McNally et al. $(1994 ; 1995)$ noted that war veterans with PTSD report more overgeneral memories than war veterans without PTSD. This suggests that overgeneral memory may be associated with particular key symptoms of PTSD, such as intrusion and avoidance symptoms (e.g., American Psychiatric Association [APA], 1994; Ehlers \& Clark, 2000; Kuyken \& Brewin, 1995).

\section{Overgeneral memory and intrusions}

In the immediate aftermath of a traumatic event, normal reactions are to experience PTSD symptoms like intrusions, avoidance or hyper-arousal (e.g., APA, 1994; Ehlers \& Clark, 2000). Generally, such symptoms fade away after some weeks, though in few individuals they persist sometimes for years (Ehlers \& Clark, 2000). One of the hallmark features of PTSD are intrusions. They are specific unwanted trauma-related memories or thoughts that continuously popup into the mind of survivors. They can take the form of "distressing recollections of the traumatic event, including images, thoughts or perceptions" (APA, 1994; p.428). In terms of the SMS theory, intrusions are regarded as a form of direct retrieval (e.g., Conway et al., 2004).

Several studies reported that intrusive memories are not only a core symptom of PTSD, but that they are also prevalent in major depression (e.g., Brewin, Hunter, Carrol, \& Tata, 1996; Carlier, Voerman, \& Gersons, 2000; Kuyken \& Brewin, 1994). Although most characteristics of intrusions are similar in PTSD and depression, their content is different (Reynolds \& Brewin, 1999). Whereas in PTSD, intrusions refer to the personal experience of (life-)threatening situations such as rape or abuse, in depression, they often refer to life events like death or illness of a loved person (Reynolds \& Brewin, 1999). Hence, 
intrusion symptoms are not only frequent in PTSD, but also in depression but their content differs across these conditions.

To sum up, overgeneral autobiographical memories and intrusions occur in depressed and traumatized individuals. Moreover, several studies have shown a positive relationship between overgeneral memory retrieval, on the one hand and specific intrusions, on the other hand (see Williams et al., 2007). For example, Kuyken and Brewin (1995) noted that particularly the presence of more specific day-to-day intrusions was associated with more overgeneral memories. In other words, the more intrusions individuals reported, the fewer specific memories they retrieved on an AMT.

Kuyken and Brewin's finding has been replicated in other samples such that a higher frequency of specific trauma intrusions in everyday life is associated with the recall of fewer specific autobiographical memories. This has been established for heterogeneous PTSD samples including assault and car accidents (Schönfeld \& Ehlers, 2006), trauma WOIl survivors from the Japanese camps (Wessel et al., 2002), victims of burn injuries (Stokes, Dritschel, \& Bekerian, 2004), victims of assault (Schönfeld et al., 2007), and depressive patients (Brewin et al., 1999; Hermans et al., 2004; Raes et al., 2006; though see de Decker et al., 2003, Kuyken, Howel, \& Dalgleish, 2006). Altogether, there is ample evidence for the association of overgeneral memory with intrusions in traumatized and depressed samples.

\section{Avoidance symptoms}

Another key symptom of PTSD is avoidance. As almost all individuals who had experienced a traumatic event suffer from intrusions, it may not be surprising that they also try to avoid those because of the accompanying distress (APA, 1994; Ehlers \& Clark, 2000). Thus, subsequent avoidance, for example through thought suppression of intrusions, often follows as a secondary reaction after a traumatic experience (e.g., Ehlers \& Clark, 2000; Wenzlaff \& Wegner, 2000). In depression, not only intrusions come to mind but also avoidance symptoms occur (e.g., Kuyken \& Brewin, 1995). Accordingly, several studies that found a relationship between intrusions and overgeneral memory, also reported a connection of overgeneral memory with avoidance symptoms in traumatized and/or depressed individuals (e.g., Brewin et al., 1999; Kuyken \& Brewin, 1995; 
Schönfeld et al., 2007; Stokes et al., 2004; Wessel et al., 2002; though see de Decker et al., 2003). Furthermore, two studies focusing on traumatized depressive adolescents (Kuyken et al., 2006) and non-clinical students (Hermans, Defranc, Raes, Williams, \& Eelen, 2005) found a significant association of overgeneral memory with avoidance characteristics, in the absence of the intrusion-overgeneral memory link.

In sum, the relative inability to retrieve specific autobiographical memories is closely associated with traumatic experiences and trauma-related symptoms (see Williams et al., 2007). In particular, overgeneral memory is related to reports of unwanted specific intrusive memories as well as to avoidance symptoms. Interestingly, overgeneral memory and intrusions both illustrate impaired autobiographical memory retrieval that negatively affect healthy functioning in everyday life. In contrast, avoidance symptoms seem more a cognitive behavioral consequence of unwanted negative intrusions and feelings related to the trauma memory. It is the link between overgeneral memory and intrusions that caught our specific attention and that will be the focus of the present dissertation.

\section{Co-existence overgeneral memory and intrusions: A paradox?}

So far, this chapter has emphasized that individuals with a major depression and/or with a history of trauma are characterized by overgeneral memories and specific intrusions (see Williams et al., 2007). Indeed, trauma experiences and trauma-related symptomatology relate to impaired autobiographical memory retrieval, which may lead to serious difficulties in everyday functioning. Exposure to highly distressing events, such as psychological traumas, regularly leads to an abundance of intrusive memories of very specific information about those experiences (e.g., McNally, 2003). At the same time, such an experience also seems to lead to a relative inability to recall specific autobiographical memories (Williams et al., 2007). In other words, the same populations that routinely show reduced memory specificity in response to cue words on an AMT are simultaneously suffering from unwanted memories of highly specific autobiographical trauma-related material (e.g., Kuyken \& Brewin, 1995; see Williams et al., 2007). How is it possible that individuals have very specific intrusions on the one hand, whereas, on the other hand, they are not able to 
retrieve specific autobiographical memories? How might this contradiction be explained?

\section{Explanations for the co-existence of overgeneral memory and intrusions}

Until now, the literature on overgeneral memory has mainly focused on its association with trauma and trauma-related symptoms (see Moore \& Zoellner, 2007; Williams et al., 2007). Many studies about overgeneral memory are based on Williams et al.'s theory (2007; see also Williams, 1996; Williams, Stiles, \& Shapiro, 1999) with a main focus on a history of trauma being the cause of overgeneral memory retrieval. This theory has its basis in the Self Memory System (e.g., Conway et al., 2004) that is described at the beginning of this chapter and it is in accordance with the cognitive theory of Ehlers and Clark (2000) about the development of PTSD. Therefore, the following paragraphs will particularly focus on Ehlers and Clark's model about PTSD and thereafter on Williams et al.'s (2007) overgeneral memory theory.

\section{Cognitive theory of PTSD}

Based on Conway et al.'s (2004) Self Memory System about autobiographical memory, Ehlers and Clark (2000) formulated a cognitive theory of PTSD explaining why some individuals develop PTSD and others do not (see Dalgleish, 2004 for an overview of other theories about PTSD). According to this model, PTSD develops in individuals who are unable to regard the trauma as time-limited, that is having a temporary impact on their future lives. The trauma experience is negatively appraised in such a way that it results in a continuous feeling of current threat. Furthermore, normal trauma-related symptoms that almost everyone experiences after a trauma, like lack of concentration or nightmares, are also negatively evaluated. For example, (temporary) lack of concentration may be interpreted as abnormal, which consequently leads to negative emotion. As a result, dysfunctional coping strategies, such as rumination or avoiding negative thoughts about the trauma, may develop and enhance PTSD symptoms. Symptoms become worse, and the trauma memory may become negatively affected.

According to Ehlers and Clark (2000), the nature of the trauma memory and unwanted intrusions about the traumatic event are a core problem of persistent 
PTSD. They argue that in individuals with PTSD the trauma memory may be poorly elaborated and incorporated into the autobiographical memory base. In terms of Conway et al. (2004), traumatic experiences are rather difficult to unify with current active goals of the working self. Usually, there are no active goals that may guide encoding and integration of traumatic experiences into the autobiographical memory knowledge base. Hence, processing during trauma may be predominantly data-driven rather than conceptual (e.g., Halligan, Clark, \& Ehlers, 2002). Accordingly, episodic memory details of the traumatic experience are stored without being contextualized in the more abstract autobiographical memory knowledge base and the conceptual self. Consequently, individuals have difficulty to intentionally retrieve the trauma memory because it has not been stored into its context in time and place, and has not been related to previous and subsequent information and other autobiographical memories. Therefore, during intentional recall, episodic details of the trauma memory may be missing and the temporal order may be disorganized (e.g., Halligan et al., 2002).

Furthermore, it becomes almost impossible to re-evaluate the traumatic event as being past, because of non existing or weak links with how life went on after the experience. Thus, the trauma memory is not - or very poorly - incorporated by the conceptual self. There is indeed empirical evidence for the theory that the trauma memory of individuals with PTSD is disorganized (e.g., see Brewin, 2007 for an overview; Halligan et al., 2002; Halligan, Michael, Clark, \& Ehlers, 2003; though see for example Berntsen \& Brewin, 2007; Porter \& Birt, 2001, Shobe \& Kihlstrom, 1997 for discussions that traumatic memories are not fundamentally different in their nature from non-traumatic memories).

Because of the disorganization of the autobiographical memory, direct retrieval based on (perceptual) internal or external cues will be more likely (Conway et al., 2004; Ehlers \& Clark, 2000; Ehlers, Michael, Chen, Payne, \& Shan, 2006). As the episodic details of the trauma memory are poorly integrated, they may become (strongly) related to current goals. To be more precisely, because there is no match between the current goals and the traumatic experience, organized storage of trauma-related episodic details is difficult and they remain active to current goals (e.g., it is not obvious how and where the traumatic memory should be categorized, so it remains active in the working self). Consequently, 
the episodic memory details from the trauma may become related to many retrieval cues and, as a result, continuously pop up into mind (i.e. intrusions). Thus, associations with retrieval cues become stronger, poor integration with the autobiographical memory knowledge base remains, and specific intrusive memories about the trauma are persistently recalled.

Taken together, Ehlers and Clark (2000) argue that the trauma memory in individuals with PTSD is disorganized. Individuals report persistent intrusions about the trauma experience and have difficulty with intentional retrieval of the trauma memory. According to Ehlers and Clark, this memory pattern may be due to predominant data-driven processing during the trauma rather than conceptual processing (e.g., Halligan et al., 2002). However, they are relatively unspoken about how overgeneral memory may develop in individuals with PTSD.

\section{Carfax model}

Williams and his colleagues (2007) recently formulated by far the most elaborate framework including three mechanisms that may explain - individually or simultaneously -overgeneral autobiographical memory. These mechanisms are capture and rumination (car), functional avoidance (fa), and impaired executive control $(\mathrm{x})$. Figure 1.3 gives a schematic illustration of how these mechanisms may lead to overgeneral memory. Basic assumptions about the organization and processes of autobiographical memory of the Carfax model are grounded in the SMS theory (e.g., Conway et al., 2004) and the model concurs with the cognitive theory of PTSD (Ehlers \& Clark, 2000).

\section{Capture and rumination}

The SMS theory assumes that in the early stage of generative retrieval, memory representations are likely to be conceptual. For example, they are abstract representations of general aspects of the self and may also include personal semantic memories such as names of friends and colleagues (Conway et al., 2004; Williams et al., 2007). Depressed individuals are inclined to continuously think of such abstract representations about their depressive symptoms, negative emotions, and the possible implications of these (i.e., rumination; Nolen-Hoeksema, 2000; Watkins, Teasdale, \& Williams, 2000). Some 
researchers have found that constant rumination (i.e., '...I have always failed at school' and '...I will never graduate') may cause an elaborate consolidation of general categoric descriptions in such a way that depressed individuals get stuck at this level of general events when they are asked to retrieve specific autobiographical memories on the AMT (Watkins \& Teasdale, 2001; Watkins et al., 2000). This is called the mnemonic interlock: individuals are 'captured' by these abstract and self-relevant representations so that the memory search is early aborted (e.g., Barnhofer, de Jong-Meyer, Kleinpass, \& Nikesh, 2002; Crane, Barnhofer, \& Williams, 2007). As a result, these conceptual (and selfrelevant) representations will be strengthened, which makes retrieval of these more likely than retrieval of specific elaborate perceptual memories. This mechanism, however, is silent about the relationship between overgeneral memory and intrusions.

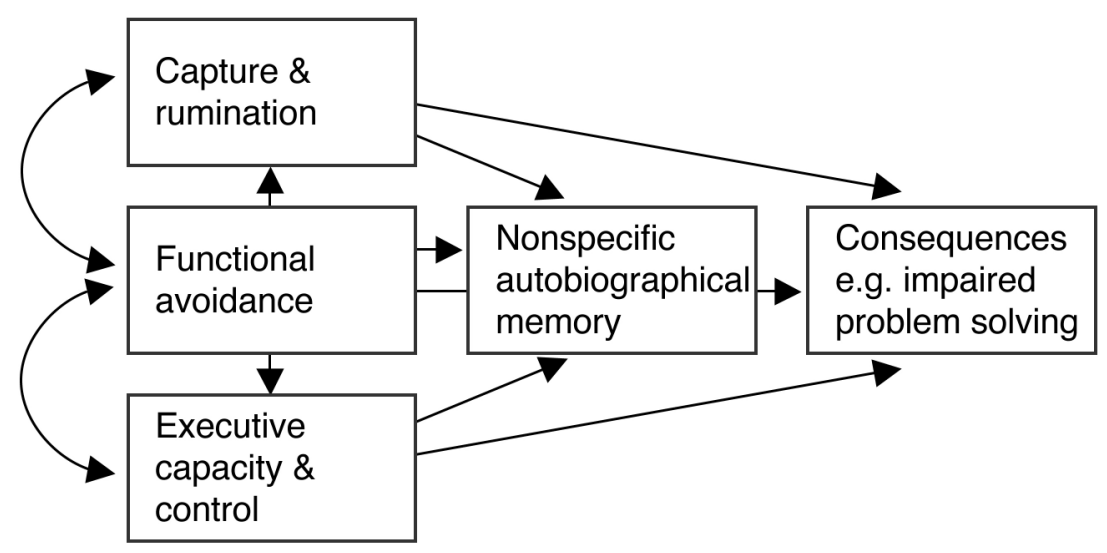

Figure 1.3. The Carfax model: three processes - capture and rumination (car), functional avoidance $(\mathrm{fa})$, and reduced executive functioning $(\mathrm{x})$ - individually or simultaneously contributing to overgeneral memory and reduced cognitive behavioral functioning (Williams et al., 2007, p.141). 


\section{Functional avoidance}

Williams et al. (2007) believe that the recall of general descriptions protects the individual from the negative affect that accompanies specific recall of traumatic events (cf., Conway et al., 2004; Ehlers \& Clark, 2000). They argue that the distress and negative affect associated with specific retrieval of the traumatic event may (gradually) lead to passive avoidance of specific trauma-related episodic information. Particularly, (episodic) sensory and perceptual details of the trauma memories usually lead to emotional distress. Early truncation of the generative search process at the level of general descriptions will be reinforced because individuals do not experience the negative mood disturbance anymore (see also Affect Regulation Hypothesis, Williams, 1996; Williams et al., 1999). After some time, this truncated retrieval process generalizes from the trauma to the whole autobiographical memory base. According to this view, overgeneral memory retrieval itself is an avoidant coping style. According to Williams et al. (2007), this functional avoidance explanation of overgeneral memory is the central part of their model.

Evidence for this view comes from correlational studies showing that overgeneral memory is positively related to self-reported cognitive avoidance symptoms (e.g., Hermans et al., 2005; Kuyken \& Brewin, 1995; see Williams et al., 2007). Furthermore, Raes and his colleagues compared students low in specific memory retrieval with students who were highly specific in their autobiographical memory recall. Participants performed a negative stressful task and afterwards indicated their levels of distress. The results showed that individuals who were low in specific autobiographical memory retrieval were less distressed after the stressful task than participants who were highly specific beforehand (e.g., Raes, Hermans, de Decker, Eelen, \& Williams, 2003; Raes, Hermans, Williams, \& Eelen, 2006). Thus, individuals who were less specific in their autobiographical memory retrieval before they participated in a stressful task demonstrated less distress afterwards.

The question arises whether this type of study really demonstrates that overgeneral memory serves an avoidant function. Given the fact that overgeneral memory typically occurs in people with a history of trauma and considering that the stressful tasks in these studies were rather mild, one could 
explain the relative lack of distress of participants with overgeneral memories to this task as an indicator that they really experienced it as a trivial event.

How does functional avoidance explain that many traumatized individuals simultaneously show overgeneral memory and highly distressing specific intrusive memories, which exactly cause the emotional distress that overgeneral memory retrieval should reduce? Possibly, reduced memory specificity is not as successful in avoiding negative affect as suggested in this functional avoidance account. Williams (2006) argues that overgeneral memory may be regarded as a dysfunctional (avoidant) coping strategy because normal mechanisms have failed. He compares it with processes such as behavioral avoidance or thought suppression that are intended to avoid negative stimuli or thoughts, but counterproductively lead to more distressing thoughts (e.g., Ehlers \& Clark, 2000; Wegner, 1994; Williams, 2006). In a similar way, overgeneral memory retrieval is intended to reduce distress. However, it fails to keep away the distressing trauma-related intrusive memories. Thus, according to this view (Williams, 2006), overgeneral memory and specific intrusions may be related because overgeneral memory retrieval is a dysfunctional avoidant coping strategy aimed at reducing the negative affect related to specific trauma memories. With such an explanation, avoidance may be underlying overgeneral memory retrieval as well as intrusive recollections.

\section{Executive capacity}

The SMS model assumes that generative retrieval is a cognitive task that requires the use of limited executive capacity (e.g., Conway et al., 2004). Depressed individuals are characterized by reduced cognitive resources and lack of initiative while performing cognitive tasks (Hertel, 2000). When autobiographical memories are retrieved via the generative route, first general descriptions (i.e., general events) are formed that may subsequently map on specific episodic memories. Lack of initiative and/or limited executive capacity may prevent the completion of such a process and may, thus, result in overgeneral memory (Williams et al., 2007). Furthermore, due to limited executive capacity, traumatized and/or depressed individuals may have difficulty with the inhibition of interfering cognitive material (e.g., intrusions) while performing a cognitive task such as autobiographical memory retrieval (Zacks \& 
Hasher, 1994). Zacks and Hasher (1994) argued that generative retrieval requires cognitive capacity to construct memories as well as to inhibit irrelevant information, such as intrusions, to protect against interference.

Following this line of argument, Dalgleish et al. (2007) hypothesized that overgeneral memory is the result of a failure to inhibit interfering information (e.g., intrusive thoughts) during the search process. They hypothesized that if overgeneral memory is an error caused by an early aborted search process due to interfering information, it would correlate to performance errors on different executive capacity tasks. The idea here is that reduced executive capacity during a generative search process consequently leads to a failure to inhibit unwanted intrusions that pop-up into mind. In a series of studies, Dalgleish et al. indeed demonstrated that overgeneral memory was related to task errors on a range of cognitive executive paradigms that had little or nothing to do with memory or the self. Thus, overgeneral memories can be conceptualized as errors on a cognitive task that requires capacity to inhibit unwanted intrusions that interfere with the search process. More specifically, overgeneral memory may be the result of a failure to inhibit unwanted intrusions that prevent generative specific autobiographical memory retrieval.

To sum up, the Carfax model proposes three separate mechanisms that may contribute individually or together to overgeneral memory retrieval in depressed and traumatized individuals. Whereas the three mechanisms - capture and rumination, functional avoidance, and impaired executive capacity - can all contribute to overgeneral memory, functional avoidance takes a central position in Williams et al.'s (2007) model because of the relatively consistent relationship between overgeneral memory and traumatic experiences (and trauma-related symptoms).

\section{Retrieval-induced forgetting as an explanation for overgeneral memory and intrusions}

A completely different approach to explain the co-occurrence of overgeneral memory and intrusions comes from Wessel et al. (2002). Perhaps, the recurrent retrieval of intrusive memories is relevant in hampering the retrieval of other autobiographical memories, which may consequently result in overgeneral memory. In this view, intrusions do not only consume limited executive capacity 
resources (e.g., Dalgleish et al., 2007; Williams et al., 2007), but they also contribute to a decreased availability of other autobiographical memories (e.g., Anderson, 2003). For example, Anderson and colleagues (e.g., Anderson, 2003; Levy \& Anderson, 2002) argued that competition during retrieval not only prohibits weaker memories to enter consciousness, but also affects the availability of these other memories in that activation levels of their memory representations are reduced.

Evidence for this suggestion comes from studies employing a retrieval practice paradigm (e.g., Anderson, Bjork, \& Bjork, 1994). Possibly, this procedure may provide an experimental analogue for the suggested paradox of specific intrusive and overgeneral memory. To be more precise, one might regard the trauma-related intrusions as winners of competition at retrieval, as they continuously pop-up into the mind. Repeated retrieval of these intrusions may consequently reduce the availability of other autobiographical memories (e.g., the specific episodic memory details become less available), which may result in an overgeneral autobiographical memory.

\section{Retrieval practice paradigm}

The retrieval practice paradigm (e.g., Anderson et al., 1994; Anderson \& Spellman, 1995) offers a way to investigate the impact of repeated retrieval of particular memories on the recall of unrehearsed memories. The original paradigm consists of three phases and relies on word stimuli (e.g., Anderson et al., 1994). During a study phase, participants learn a certain number of category-exemplar associations, each category consisting of several exemplars (e.g., Fruit-Banana; Fruit-Orange; Garden-Tree). Subsequently, participants are directed to retrieve half of the studied exemplars from half of the categories by completing category-stem pairs (e.g., Fruit-Ba_). In this retrieval practice phase, the critical exemplars are practiced three times. After a retention interval of $20 \mathrm{~min}$, participants engage in a cued recall test. They are cued with a category name (e.g., Fruit, Garden) and asked to recall all of the exemplars from all of the categories that were presented at any point in the experiment. There are thus three types of exemplars: practiced exemplars (Fruit-Banana), unpracticed exemplars from the practiced categories (Fruit-Orange), and unpracticed exemplars from unpracticed categories (Garden-Tree). 
Although the primary interest of this paradigm is not in the practiced exemplars, recall for practiced exemplars (Fruit-Banana) is typically enhanced. This is called the retrieval practice effect. The result of particular interest is that fewer unpracticed exemplars from the practiced categories (Fruit-Orange) are recalled than unpracticed exemplars from unpracticed categories (Garden-Tree). This outcome is termed retrieval-induced forgetting (Anderson et al., 1994). At first sight, this might be counterintuitive as on the basis of traditional spreading of activation models, retrieval practice on Fruit-Banana should enhance performance of Fruit-Orange (e.g., Anderson \& Spellman, 1995). However, if retrieval of Fruit-Banana activates processes that inhibit pre-defined unpracticed exemplars, it may not be surprising that recall of the unpracticed Fruit-Orange is impaired (Anderson \& Spellman, 1995).

The retrieval-induced forgetting effect has been replicated with a variety of stimulus materials such as words, visual material, and semantic material (for a review see Levy \& Anderson, 2002). According to Anderson et al. (1994), this effect is caused by inhibition of competing exemplars that are associated with the same (retrieval) cue as the retrieval-practiced exemplars. Experimental evidence for this inhibition theory comes from Anderson and Spellman (1995) who demonstrated that the reduced availability of unpracticed exemplars from the practiced categories (Fruit-Orange) was cue independent (see also Saunders \& MacLeod, 2006). To be more specific, the recall of the unpracticed exemplar Orange was still impaired even when the retrieval cue was another one than Fruit, for example Color. However, several studies (e.g., Camp, Pecher, \& Schmidt, in press; Williams \& Zacks, 2001) have failed to replicate this cue independency. Therefore, other explanations such as interference theory are put forward to account for the retrieval-induced forgetting effect (see MacLeod, Dodd, Sheard, Wilson, \& Bibi, 2003 for a review).

\section{Emotional and autobiographical material}

Important for the present purpose is the question whether retrieval-induced forgetting also occurs in more ecologically valid situations using emotional and autobiographical memory material. For example, repetitive police interviews about particular aspects of a crime may result in forgetting other details related to that same offence. Indeed, several studies (MacLeod, 2002; Saunders \& 
MacLeod, 2002; Shaw, Bjork, \& Handal, 1995) have demonstrated that repeated questioning enhances participants' memory for the rehearsed aspects, but promotes forgetting of the unrepeated details. In such studies, the material itself is not emotional per se. However, the context in which participants repeatedly retrieved information is stressful to some extent (e.g., a story of a burglary).

Additionally, some studies have demonstrated retrieval-induced forgetting for emotional material (e.g., Amir, Coles, Brigidi, \& Foa, 2001; Barnier, Hung, \& Conway, 2004). For example, in an intriguing study, Barnier et al. (2004) have shown that retrieval practice of emotional autobiographical memories impaired recall of unpracticed autobiographical memories that were related to the same emotional cue (e.g., sickness, happy). The results demonstrated that repeated retrieval of some autobiographical memories induced forgetting of other memories that were related to the same retrieval cue. Taken together, these findings do not only imply that retrieval-induced forgetting can be generalized to emotional material, but also to more complex episodic material like autobiographical memories.

\section{Intrusions versus overgeneral memory}

The above-mentioned findings suggest that retrieval-induced forgetting may underlie remembering and forgetting of emotional and autobiographical memories in that repeated retrieval of some emotional and autobiographical memories may reduce the availability of other related memories. These findings have led to the present idea that retrieval-induced forgetting may provide an experimental analogue explaining the co-occurrence of intrusions and overgeneral memory (e.g., Wessel et al., 2002). In other words, due to constant retrieval, persistent intrusions may contribute to a decreased availability (i.e., inhibition) of the episodic memory details of other autobiographical memories (e.g., Anderson, 2003). This may ultimately result in an overgeneral autobiographical memory. 


\section{Aim and outline of the present dissertation}

The central aim of this dissertation is to explore how the positive association of overgeneral memory with intrusions in the context of trauma can be accounted for. To this end, the first study described in Chapter 2 examined whether the well-replicated correlation between the two phenomena in (sub)clinical samples (e.g., Williams et al., 2007) can be reproduced in a non-clinical sample. Thereafter, in Chapters 3 and 4 the thesis focuses on the merits of a retrievalinduced forgetting account to explain the positive relationship between overgeneral memory and intrusions (e.g., Anderson, 2003; Wessel et al., 2002). In Chapters 5 and 6, two studies are described that have tested hypotheses derived from the CaRFaX model (e.g., Williams et al., 2007) to clarify the association between intrusions and overgeneral memory. Finally, Chapter 7 reports a prospective study that explored the role of overgeneral memory in predicting later posttraumatic stress symptoms.

Overgeneral memory, intrusions, and avoidance in a non-clinical sample

The first study (Chapter 2) was set up to replicate the correlation between intrusions, avoidance, and overgeneral memory in a non-clinical sample. So far, these associations have been primarily found in clinical samples of depressed and traumatized individuals or in individuals with a history of trauma (see Williams et al., 2007). However, intrusive memories are not uncommon in healthy samples (e.g., Berntsen, 2001; Bywaters, Andrade, \& Turpin, 2004), nor is the tendency to avoid unwanted thoughts (Wenzlaff \& Wegner, 2000). Furthermore, reduced memory specificity has also been found in non-clinical student samples (e.g., Merckelbach, Muris, \& Horselenberg, 1996). Therefore, one may expect that the link between overgeneral memory, intrusions, and avoidance of aversive experiences can be reproduced in a non-clinical sample. To identify which processes or factors may underlie the link between these phenomena, it would be relevant to determine whether or not such an association is exclusively found in (sub)clinical samples. What is more, we first wanted to validate the idea of individual differences on the AMT in a non-clinical group before conducting studies in which we wanted to manipulate autobiographical memory (e.g., Chapters 5 and 6). 


\section{Retrieval-induced forgetting}

Chapters 3 and 4 describe five studies that investigated to what extent retrievalinduced forgetting may be an (experimental) analogue for the co-occurrence of intrusive memories and overgeneral memory retrieval. The general idea behind this account is that repeated retrieval of trauma-related intrusive memories reduces the availability of other autobiographical memories, which may ultimately result in overgeneral memory (Wessel et al., 2002).

The two studies in Chapter 3 elaborate further on the findings of Barnier et al. (2004), who found that repeated retrieval of some emotional autobiographical memories reduced retrieval of other unpracticed memories that were related to the same retrieval cues. Our aim was to extend these results to positive and negative autobiographical memories while using other types of retrieval cues than did Barnier et al. (2004). The principal research question was whether repeated retrieval of some autobiographical memories would induce forgetting of other unpracticed autobiographical memories that are or are not of the same emotional valence. The role of valence may potentially be a relevant issue because intrusions in the context of trauma are mainly negative, whereas overgeneral memories may be retrieved to positive and negative cue words.

Elaborating further on the findings of Chapter 3, the three studies described in Chapter 4 aimed to generalize retrieval-induced forgetting to emotionally negative slides. As intrusions tend to primarily contain visual and other perceptual details (Conway et al., 2004), in this study we investigated whether retrieval-induced forgetting would also occur with emotionally and negatively valenced slides. Such a finding would also be interesting in the broader context of forgetting of emotional material and other PTSD-like phenomena, such as dissociative amnesia (e.g., Brewin \& Andrews, 1998; Holmes et al., 2007)2 Rather than examining the effect of repeated retrieval of some memories on other unrehearsed memories (i.e., Chapter 3 ), the effect of repeated retrieval of some details of a negatively valenced scene on unrehearsed details of that

\footnotetext{
2 Some authors (e.g., Brewin \& Andrews, 1998; Holmes et al., 2007) believe that traumatized participants are not able to retrieve particular details of their traumatic event because they are too emotional and aversive.
} 
same scene was investigated. Furthermore, in the aftermath of a traumatic experience visual details may be the primary focus of police interviews in which some aspects of the experience may be repeatedly retrieved whereas others are not (e.g., MacLeod, 2002). This may have harmful effects on the retrieval of unrehearsed details.

\section{Functional avoidance}

In contrast to the retrieval-induced forgetting account, the Carfax model (e.g., Williams et al., 2007) regards the relationship between overgeneral memory and intrusions as having a mutual origin in that both phenomena may be the result of a possible third variable, such as functional avoidance or impaired executive functioning. For example, if avoidance is the underlying process, traumatized individuals may start to retrieve fewer specific memories because they want to avoid the negative affect that is related to intrusions. However, this may be a counterproductive strategy that subsequently may result in even more intrusive memories (Williams, 2006). Accordingly, both overgeneral memory and intrusions may be the result of (functional) avoidance.

Chapter 5 describes a study that aimed to investigate whether avoidance would be an underlying factor of both overgeneral memory and intrusions. In a student sample, intrusions were elicited by asking participants to suppress thoughts of their most negative experience (e.g., Wegner, 1994). The idea behind this manipulation is that active thought-suppression counterproductively leads to more thoughts about the suppressed event directly after an active suppression period (e.g., white bear effect, Wegner, 1994). Thus, the effect of suppression on subsequent intrusions and autobiographical memory specificity was explored. To be precise, in Chapter 5 we tested the prediction that thought suppression of ones most negative experience would lead to more intrusions as well as to the retrieval of fewer specific autobiographical memories on an AMT.

\section{Two retrieval routes}

Chapter 6 describes a study testing the hypothesis that intrusions and overgeneral memory are both retrieved via different retrieval routes that operate at different times. In the Self Memory System, Conway et al. (2004) describe that intrusions are retrieved via a direct retrieval process (i.e., spontaneous), 
whereas autobiographical memory retrieval on an AMT results from a generative search process (i.e., deliberate). Furthermore, Williams et al. (2007) note that autobiographical memory retrieval on an AMT is a generative search process that is disrupted. Thus, the co-occurrence of overgeneral memory and specific intrusions may be explained by the fact that the direct retrieval process is still intact, whereas the generative retrieval process is disturbed (e.g., Conway et al., 2004).

In order to test this account, a new version of the AMT that promotes direct retrieval (e.g., Williams, Chan, Barnhofer, Eade, \& Healy, 2006; Williams, Healy, \& Ellis, 1999) was developed and compared to the standard AMT that has been designed to promote generative retrieval. If the generative retrieval process has been impaired but the direct retrieval process is still intact, then traumatized individuals displaying intrusions would be predicted to exhibit overgeneral memory on the standard AMT, but not on the new AMT that promotes direct retrieval.

Overgeneral memory as a predictor of later posttraumatic stress symptoms

Prior studies have shown that overgeneral memory may be a vulnerability factor to develop depressive symptoms after experiencing a negative stressful event (e.g., Mackinger et al., 2000; van Minnen et al., 2005). Because PTSD symptoms are a relatively common outcome after traumatic experiences (e.g., APA, 1998; Shalev et al., 1998) and are also related to overgeneral memory (e.g., Moore \& Zoellner, 2007; Williams et al., 2007), we were interested in whether overgeneral memory would also predict later PTSD symptoms. There is, indeed, one study that demonstrates that traumatized individuals with ASD related to a motor vehicle accident, who displayed an overgeneral memory, reported more PTSD symptoms six months later (Harvey et al., 1998).

Following the methodology of prior studies (e.g., Mackinger et al., 2000; van Minnen et al., 2005), Chapter 7 describes a study investigating whether pretrauma overgeneral memory is a vulnerability factor for later posttraumatic stress symptomatology. The study included a sample of hospitalized women with pregnancy complications. The AMT was administered prepartum, next to measures of trauma history, pre-trauma mental health, executive functioning, and initial posttraumatic and depressive symptoms (see Williams et al., 2007). 
Six weeks after giving birth, outcome measures of posttraumatic stress symptomatology were administered.

To end, Chapter 8 summarizes the findings of the current research and places them into the theoretical context of today's literature. Methodological topics are discussed as well as directions for future research. Chapter 8 ends with the main conclusions that can be drawn from the present results. 



\title{
Intrusions, avoidance and overgeneral memory in a non-clinical sample
}

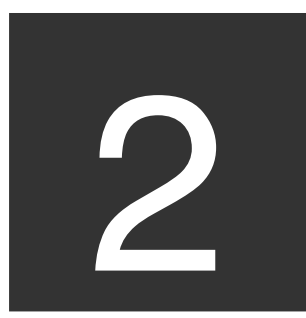

\author{
B.J.A. Hauer, I. Wessel, H. Merckelbach
}

Adapted from: Clinical Psychology and Psychotherapy (2006: 13, 264-268)

Previous studies showed a positive relationship between intrusions, effortful avoidance and overgeneral memory in people suffering from (mild) depression or PTSD. The purpose of the present study was to investigate these relations in a non-clinical sample. As part of a mass testing session, a written version of the Autobiographical Memory Test was administered to 175 first-year psychology students. Furthermore, intrusions and avoidance symptoms were measured with the Impact of Event Scale (IES; Horowitz, Wilner, \& Alvarez, 1979), and depressive symptoms with the Beck Depression Inventory (BDI; Beck, Ward, Mendelson, Mock, \& Erbaugh, 1961). The data demonstrated a significant correlation between avoidance symptoms and categoric memory to negative cue words. This relationship remained significant after controlling for depressive symptoms. These results concur with other findings that overgeneral memory tends to be related with symptoms of effortful avoidance. 


\section{Introduction}

Over the past 20 years, numerous studies have shown a relationship between autobiographical memory specificity and psychopathology (see for an overview van Vreeswijk \& de Wilde, 2004). These studies demonstrated that particularly people with depression (e.g., Kuyken \& Brewin, 1995) or posttraumatic stress disorder (PTSD; e.g., McNally, Lasko, Macklin, \& Pitman, 1995) have difficulty recalling specific memories of personally experienced events that happened at a particular time and place (i.e., 'last week when I had dinner with my sister'). This relative inability to recall specific autobiographical memories has been termed overgeneral memory (Williams \& Broadbent, 1986). Overgeneral memory can be either categoric, referring to a broad class of events (i.e., 'every time when I go to work'), or extended, pertaining to events lasting longer than one day (i.e., 'my vacation in France'). Overgeneral memory is measured with the Autobiographical Memory Test (AMT; see van Vreeswijk \& de Wilde, 2004). The total number of categoric (and extended) memories on the AMT serves as a measure of overgeneral memory (i.e., lack of autobiographical memory specificity).

In the literature, one recurrent theme is that aversive life events tend to play a central role in the origin of overgeneral memory. Several studies showed a positive relationship between traumatic experiences and reduced autobiographical memory specificity (e.g., de Decker, Hermans, Raes, \& Eelen, 2003; Henderson, Hargreaves, Gregory, \& Williams, 2002). However, researchers disagree about the precise role of traumatic events. Is the experience of an aversive event a prerequisite to develop overgeneral memory (Williams, Stiles, \& Shapiro, 1999)? Or do other factors related to these experiences (e.g., depression) contribute to overgeneral memory (Wessel, Merckelbach, \& Dekkers, 2002)? The fact that not everyone who experienced a traumatic event will develop overgeneral memory (e.g., Harvey, Bryant, \& Dang, 1998; McNally et al., 1995) implies that there are more antecedents. Some researchers also suggest that other variables may mediate or moderate the relationship between trauma and overgeneral memory (e.g., Raes, Hermans, Williams, \& Eelen, 2005). 
There is indeed evidence indicating that how one copes with trauma may be an important contributor. For example, Raes et al. (2005) found that lower levels of support received after abuse experience tended to relate with reduced autobiographical memory specificity. Rumination is also likely to moderate the relation between trauma and overgeneral memory (Raes et al., 2006). Other interesting candidates are level of intrusions and/or effort to avoid memories of previous traumatic events. Relying on clinical samples, several studies (e.g., Kuyken \& Brewin, 1995; Raes et al., 2006; Stokes, Dritschel, \& Bekerian, 2004; Wessel et al., 2002; but see Kremers, Spinhoven, \& van der Does, 2004) demonstrated positive correlations between overgeneral memory, intrusions and effort to avoid memories of traumatic events (as indexed by the Impact of Event Scale; Horowitz et al., 1979). Williams (2006) suggested that having intrusions and/or effortful avoidance of memories about earlier trauma may contribute to overgeneral memory because they reduce the available executive resources to search for specific memories. Furthermore, he suggested that previous trauma may cause overgeneral memory through avoidance of (emotion related) specific memories.

So far, studies addressing overgeneral memories have primarily relied on clinical samples. And a limited number of studies demonstrated that normal samples are relatively specific (e.g., Merckelbach, Muris, \& Horselenberg, 1996). However, intrusive memories are not uncommon in healthy samples (e.g., Berntsen, 2001; Bywaters, Andrade, \& Turpin, 2004). Also, the tendency to avoid unwanted memories is not unusual in non-clinical samples (Wenzlaff \& Wegner, 2000). Therefore, one would expect that the link between overgeneral memory on the one hand and intrusions and avoidance of aversive experiences on the other hand can be reproduced in a non-clinical sample. This is the goal of the current study as, up to now, this issue has not been addressed. The present sample is a large group of healthy first year psychology students. We hypothesized positive correlations between intrusions and avoidance of traumatic memories and overgeneral memory, independent of depressive symptoms. 


\section{Method}

\section{Participants}

A sample of 175 first-year psychology students (53 men) participated in a mass testing session. Mean age was $19.54(S D=4.20$; range: $17-36)$. Participation was voluntary and participants received $€ 5$ for their participation. The study was approved by the standing ethical committee of Maastricht University. Due to missing data, 3 participants were left out for the analyses, leaving a final sample of 172 participants.

\section{Materials}

\section{Autobiographical Memory Test}

The AMT (Williams \& Broadbent, 1986) consisted of five positive and five negative cue words. The AMT was administered in a written format. A written version of the AMT was administered in previous studies yielding similar results as the oral version (Henderson et al., 2002; Hermans, Defranc, Raes, Williams, \& Eelen, 2005; Raes, Hermans, de Decker, Williams, \& Eelen, 2003; Wessel, Meeren, Peeters, Arntz, \& Merckelbach, 2001). Here, procedure and words were taken from Raes et al. (2003). Booklets were constructed with one cue word on each page. The first two pages showed the practice words grass, and bread. The next pages had the test words, alternating positive and negative cues: confidence (trust), scared, pleasurable, angry, courage, sad, calm (at ease), bold, surprised, and stupid ${ }^{\beta}$. Participants were asked to write down a specific memory for each cue word. A specific memory was defined as a memory referring to a particular event that had happened at a particular time and place. Participants had 60s to write down their response. If this time limit was reached, participants were asked to turn their pages and respond to the next cue word.

Responses to the AMT words were rated by an independent rater. Each response was coded as specific, general categoric, general extended, no

\footnotetext{
${ }^{3}$ In Dutch these words are: vertrouwen, bang, prettig, boos, moed, droevig, gerust, brutaal, verrast, lomp.
} 
memory (i.e., an association), or no response. The specificity score ranges between 0 and 10. A second rater scored a random sample of $20 \%$ of the AMT responses. Intraclass Correlation Coefficients (ICC; see Fleiss, 1986) were computed for the dependent variables. For the number of specific memories, inter rater agreement was .81, and for the number of overgeneral memories 68 .

\section{Impact of Event Scale (IES)}

The IES (Brom \& Kleber, 1985; Horowitz et al., 1979) measures the level of spontaneous intrusions and avoidance within the week prior to assessment about a (highly) stressful event. First, participants wrote down a short description of the most aversive event in their lives. They also indicated when it had happened. Then, participants scored 15 items with respect to this event on a 4-point scale (weighted $0,1,3,5$; anchored $0=$ not at all and $5=$ often), asking to what extent participants had spontaneous intrusions and avoidance symptoms within the previous week. Sample items are "I thought about it while I didn't want to" and "I tried not to talk about it". The total score ranges from 0 to 75. The psychometric properties of the total scale are good $(a=.71)$, as well as for the intrusion $(a=.72)$ and avoidance $(a=.66)$ subscales.

Beck Depression Inventory (BDI)

The BDI (Beck et al., 1961; Bouman, Luteijn, Albersnagel, \& Ploeg, 1985) consists of 21 items all containing four statements describing depressive symptoms in increasing severity (scored $0-3$ ). Illustra tive items are 'I have less energy than previously' and 'I have less self confidence'. The total score (range: $0-63$ ) reflects self-reported depressive symptoms over the past week. The psychometric properties are good with Cronbach's $a$ between .79 and .90 .

\section{Procedure}

The AMT was first administered as part of the mass testing session. The experimenter read aloud the instructions (compare Raes et al., 2003) and then the two practice words were presented and discussed. Next, the ten stimulus words were given. Participants wrote down their memories to each stimulus word. After the AMT, participants filled in several questionnaires, including the $\mathrm{BDI}$ and the IES. All questionnaires were administered in a fixed order during 
the same session. The BDI preceded the IES. At the end of the session, participants were thanked for their participation, debriefed, and given their money.

\section{Results}

Raw scores on the AMT were used as the dependent measure. The mean number of reported specific memories was $7.61(S D=1.94)$. The mean number of extended, categoric, no memories, and omissions was .99 ( $S D=1.20)$, .27 $(S D=.64), .27(S D=.58)$, and $.41(S D=.87)$. The mean score on the BDI was $6.03(S D=5.40$; Range: 0 - 35). Furthermore, the mean score on the IES was 16.78 ( $S D=15.80$; Range: 0 - 67). For the intrusion and avoidance subscales mean scores were 8.21 ( $S D=8.14$; Range: 0 - 35) and 8.57 ( $S D=8.80$; Range: 0 - 38) respectively.

We first consider the type and recency of the aversive event. Eighty participants (46.5\%) reported death or illness of beloved persons as their most aversive event. Twenty-two participants (12.8\%) recalled threat to self or beloved person and 28 participants (16.3\%) reported interpersonal problems (e.g., divorce of parents, the end of a relationship or friendship). Furthermore, 15 persons (8.7 $\%$ ) reported having experienced or witnessed an accident, 7 participants $(4.1 \%)$ recalled events that did not fit into the other categories, and 20 participants $(11.6 \%)$ did not write down their most aversive event. Twenty-nine participants $(16.9 \%)$ reported not having any intrusions or avoidance symptoms within the past week about their most stressful experience. The mean period of time between time of testing and the occurrence of the aversive event was 49.59 ( $S D$ $=44.34$ ) months. Thus, on average, the stressful event happened around four years before time of testing.

The data demonstrated significant positive correlations between BDI and the IES total scale, $r(172)=.36, p<.001$, the intrusion, $r(172)=.32, p<.001$, and avoidance, $r(172)=.35, p<.001$, subscales. Recency of the aversive experience also significantly correlated with the IES total scale, $r(156)=-.32, p$ $<.001$, the intrusion, $r(156)=-.26, p=.001$, and avoidance, $r(156)=-.32, p<$ 
.001 , subscales of the IES ${ }^{4}$. These correlations demonstrated that a more recent aversive experience (fewer months ago) was related to higher selfreported intrusions and effortful avoidance.

Pearson correlation coefficients were computed between the number of specific memories to positive $(M=3.66 ; S D=1.15)$ and negative $(M=3.95 ; S D=1.16)$ cue words and the level of intrusions and avoidance. In both cases, the number of specific memories was not related to the IES intrusion, both $r$ 's $(172)<.04$ .05 , p's $>.60$, and avoidance, both $r$ 's $(172)<.06 .05$, p's $>.30$, subscales. Looking at the number of categoric memories, a significant correlation emerged between categoric memories to negative cue words with the IES avoidance subscale, $(M=.17 ; S D=.45), r(172)=.16, p<.04$, and not in response to positive cue words, $(M=.10 ; S D=.40), r(172)=-.03, p>.70$. Furthermore, correlations between the IES intrusion subscale, BDI and categoric memory remained non-significant. As self-reported avoidance was both significantly related to depressive symptoms and to the recency of aversive events, part correlations were computed. The relation between avoidance and categoric memory to negative cue words remained significant after controlling for depressive symptoms, $r(171)=.15, p=.05$, and for recency of the aversive event, $r(156)=.16, p<.04$.

\section{Discussion}

The aim of this study was to investigate the link between intrusions and avoidance of memories of aversive experiences and overgeneral memory in a non-clinical sample. The present data showed that avoidance symptoms were related to categoric memories to negative cue words. This relationship was independent of current depressive symptoms and recency of the stressful experience. This result extends previous studies demonstrating that reduced autobiographical memory specificity was related to various measures of effortful avoidance (Hermans et al., 2005; Raes et al., 2006). It also corresponds with other data showing that avoidance symptoms were related to overgeneral memory in clinical samples (e.g., Kuyken \& Brewin, 1995; Stokes et al., 2004;

\footnotetext{
${ }^{4}$ Here, sample size is smaller as not all participants filled in the date of the event.
} 
Wessel et al., 2002). However, in contrast to our expectations and previous literature, the data did not demonstrate a relationship between intrusions and overgeneral memory.

The present findings suggest that higher IES avoidance scores are associated with overgeneral memory. Up to now, this was not demonstrated in a nonclinical sample with participants reporting a variety of major and minor aversive events. Henderson et al. (2002) did find a significant relation between selfreported Childhood Sexual Abuse (CSA) and reduced autobiographical memory specificity in a non-clinical sample. But, they did not find a relationship between IES scores (all filled out in relation to CSA) and autobiographical memory specificity. Previous clinical studies did show correlations between IES scores and overgeneral memory, both in participants who reported a variety of traumatic events (e.g., Raes et al., 2006), and in samples where participants filled out the IES with respect to the same experience (e.g., CSA, Kuyken \& Brewin, 1995; motorcycle accidents, Harvey et al., 1998). However, in these (sub)clinical samples correlations tended to be higher than in the present group. This might not be surprising as symptom severity, type of traumatic experiences, and the number of overgeneral memories also tended to be higher than in this non-clinical sample. When further interpreting the current results, we should note that past history of depression was not taken into account. And the absolute number of categoric memories was rather low. This is, however, similar to preceding studies showing that, overall, non-clinical samples tend to be quite specific in their autobiographical memories (e.g., Hermans et al., 2005; Merckelbach et al., 1996).

Taken together, the current results seem to agree with the idea that not trauma per se but how one copes with it may play a more important role in the origin of overgeneral memory (e.g., Raes et al., 2006). This also concurs with the recently revised theory of Williams and his colleagues, arguing that there are three processes contributing to overgeneral memory (Williams, 2006). Overgeneral memory may develop a) when retrieval is hijacked by other selfrelevant processing; b) when retrieval is early aborted to avoid emotional distress; c) when retrieval is affected due to reduced available executive resources (see Williams, 2006 for a more detailed description). 
The present results should be regarded as a lead to further analyze the relationship between intrusions, avoidance and overgeneral memory. Future studies should undertake attempts to directly address the causal relationship between these phenomena. For example, experiments in which participants suppress their most negative experience (i.e., Wegner, Carter, White, \& Schneider, 1987) could be a useful avenue to explore the effect on subsequent overgeneral memory. In addition, prospective studies on intrusions, avoidance and overgeneral memory could shed more light on the temporal order of these phenomena. 



\title{
Retrieval-induced forgetting of autobiographical memory details
}

\author{
I. Wessel, B.J.A. Hauer
}

Cognition and Emotion (2006: 3/4, 430-447)

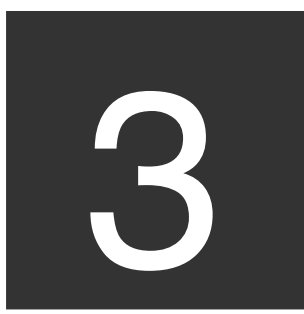

Several studies suggest that intrusive and overgeneral autobiographical memory, are correlated. Thus, paradoxically, in some patients a hyperaccessibility of memory for one (series of) event(s) goes hand in hand with a scarcity of memories for other personal experiences. This clinical observation is reminiscent of the laboratory phenomenon of Retrieval-Induced Forgetting (RIF). RIF refers to the finding that repeatedly recalling some experimental stimuli impairs subsequent recall of related (i.e., tied to the same retrieval cue) stimuli. RIF of emotional autobiographical memories might provide an experimental model for the clinical memory phenomena in question. The present paper reports two experiments that explored the merits of applying the retrieval practice paradigm to relatively broad categories of autobiographical memories. Both studies found a significant RIF-effect in that practiced memories were recalled better than unrelated unpracticed (baseline) memories. In addition, unpracticed memories that were related to the practiced memories were recalled more poorly than baseline memories. Implications of these findings for modeling the co-occurrence of intrusive and overgeneral memories are discussed. 


\section{Introduction}

Overgeneral autobiographical memory (OGM) refers to the phenomenon that some patients are relatively unable to come up with memories of personally experienced events that are tied to a specific time and place (Williams, 1996). In contrast, intrusive memory (IM) is characterized by the unbidden occurrence of recollections of a personally experienced event that interrupts daily activity and is difficult to control (e.g., Brewin, Christodoulides, \& Hutchinson, 1996). Interestingly, there is evidence that IMs and OGMs are correlated in various clinical samples (Brewin, Reynolds, \& Tata, 1999; Brewin, Watson, McCarthy, Hyman, \& Dayson, 1998; Kuyken \& Brewin, 1995; Stokes, Dritschel, \& Bekerian, 2004; Wessel, Merckelbach, \& Dekkers, 2002). Although some studies failed to find such a link (de Decker, Hermans, Raes, \& Eelen, 2003; Henderson, Hargreaves, Gregory, \& Williams, 2002; Hermans, van den Broeck, Belis, Raes, Pieters, \& Eelen, 2004), these observations raise the question of what mechanisms might be involved. An account that specifically addresses the role of IM in OGM holds that the occurrence (Brewin et al., 1999) or the avoidance (Kuyken \& Brewin, 1995) of intrusions occupies working memory and thus leaves fewer resources for retrieval of other memories. As Brewin and colleagues (1999, p.515) put it: "this interference may simply result from the presence of other information competing for limited space in working memory". Thus, IMs would easily get in the way of other autobiographical memories (AMs).

An alternative account would be that IM not only consumes working memory resources, but also contributes to a decreased availability for recall of other competing AMs. In the experimental memory literature, it has been suggested (Anderson, 2003; Levy \& Anderson, 2002) that the effects of competition at retrieval go further than merely prohibiting weaker memory representations to access consciousness. Retrieval competition may also affect the availability of these other memories in that activation levels of their representations are reduced (i.e., inhibition). Evidence for this suggestion comes from studies employing the retrieval practice paradigm. In the typical study (Anderson, Bjork, \& Bjork, 1994), participants learn a list of category names (Fruit, Furniture), each in conjunction with up to six different exemplars (e.g., Fruit-Apple; Fruit- 
Strawberry; Furniture-Chair, Furniture-Closet). In a later retrieval practice phase, the crucial cued recall manipulation (e.g., Fruit-Ap__) induces participants to repeatedly retrieve only half of the exemplars of only half of the categories. In a later category cued recall test, participants are instructed to again retrieve all exemplars of the earlier study list. Of course, results typically show a practice effect such that recall of practiced exemplars (Fruit-Apple) is enhanced relative to exemplars from unrelated, unpracticed categories (Furniture). More interestingly however, recall of exemplars from practiced categories that were not part of the retrieval practice phase (i.e., unpracticed related exemplars, e.g. Fruit-Strawberry) is impaired relative to the exemplars from unrelated, unpracticed categories (Furniture). This latter effect has been dubbed RetrievalInduced Forgetting (RIF). One interpretation (e.g., Anderson, 2003) is that RIF signals inhibition of the representations of related unpracticed exemplars in long-term episodic memory. Since the initial demonstration of RIF employing category - exemplar pairs (Anderson et al., 1994), the effect has been found in a number of subsequent studies employing various types of stimuli (e.g., homographs, propositions, geometric shapes, slides, stories) and themes (e.g., personality traits, burglaries, see for an overview Levy \& Anderson, 2002). Thus, there is good evidence in the experimental psychology literature that repeated retrieval of some memories has a detrimental effect on recall of related memories.

The phenomenology of RIF bears resemblance to observations in the clinical literature that IMs and OGMs are correlated. That is, IM involves the frequent retrieval of certain memories whereas OGM refers to a relative inability to come up with specific memories of other personally experienced events. This raises the question whether similar mechanisms as in RIF studies are involved here. Perhaps then, the retrieval practice paradigm might provide an experimental analogue for the clinically observed link between intrusive and overgeneral memories. Interestingly, the method was recently successfully applied to AMs. Barnier and colleagues (Barnier, Hung, \& Conway, 2004) asked their participants to generate AMs in response to affectively laden cue-words (e.g., horrified, happy). Each word was a cue to four unique AMs, thus resembling the semantic categories with several exemplars that were employed in earlier studies. Next, the AMs were used in a retrieval practice experiment. The results 
showed that fewer unpracticed related than unpracticed unrelated memories were recalled. Thus, RIF was observed for complex, emotional memories and this may serve as the starting point for further inquiries of whether RIF might be involved in psychopathological memory phenomena such as the co-occurrence of IMs and OGMs. Of course, in its original form the basic retrieval practice procedure is artificial. Our goal is to modify the paradigm in a systematic fashion such that ultimately, it more closely resembles the real-life observations of interest. The present paper reports two experiments that were intended as a first step in that direction.

\section{Experiment 1}

An earlier study in elderly concentration camp survivors (Wessel et al., 2002), showed that scores on a measure of intrusive memories (Impact of Event Scale, IES; Horowitz, Wilner, \& Alvarez, 1979) were correlated with fewer specific AMs in response to negative cues, but not in response to positive cues (see Kuyken \& Brewin, 1995, for a similar finding). As the IES specifically targets IMs of negative shocking events, the question rises whether the accompanying lack of AM specificity in response to negative cues may be interpreted as a RIF-like phenomenon. One problem with such an interpretation is that these earlier studies measured AMs using a broad range of cues, whereas retrieval practice studies rely on relatively narrow categories (e.g., Anderson et al., 1994; Barnier et al., 2004). Therefore, experiment 1 explored the merits of applying the retrieval practice paradigm to the relatively broad categories of "positive" and "negative" AMs.

In order to explore this, healthy participants were asked to generate both positive and negative AMs, each memory pertaining to a unique event. Several weeks later, these AMs were used as stimuli in a retrieval practice experiment. The expectation was that overall, recall of unpracticed AMs that were related to practiced AMs would be impaired compared to unpracticed unrelated AMs. In addition, it was expected that the retrieval practice manipulation would equally affect recall of positive and negative AMs. That is, if it is repeated retrieval that affects the recall of other AMs, then this should occur for related memories of the same category, irrespective of the valence of that category. 


\section{Method}

\section{Participants}

Participants were 48 second-year Psychology students $(9$ men) at the University of Maastricht. At the time of testing they had not had any courses on memory. They received $€ 15$ for participating. The experiment was approved by the ethical committee of the department of psychology at Maastricht University.

\section{General overview of procedure}

The study consisted of two sessions. In session 1, AMs were collected in order to serve as idiosyncratic stimuli in session 2 . Session 2 was a Retrieval Practice (RP) experiment consisting of a 1) study phase; 2) RP phase; 3) distracter phase; and 4) test phase.

\section{Materials}

\section{AM collection}

Booklets were constructed consisting of 12 pages with "negative memory" and 12 pages with "positive memory" printed at the top of the pages. Each page had the words "where" "when" "who" and "what" printed at the left margin. At the bottom of the page, three 11-point Likert scales asked for emotional intensity (anchored $0=$ not at all intense and $10=$ extremely intense), negative valence (anchored $0=$ neutral and $10=$ extremely negative) and positive valence (anchored $0=$ neutral and $10=$ extremely positive) of the memories. Half of the booklets asked for 12 negative AMs first, whereas the other half of the booklets began asking for 12 positive AMs.

\section{Study phase}

Ten positive and 10 negative AMs of comparable emotional intensity were selected from the session 1 booklets. In the study booklets these AMs were typed on separate pages in random order. The appropriate valence category label (i.e., either "positive memory" or "negative memory") was written at the top of each page. 


\section{RP phase}

For RP booklets, a set of five different AMs was selected from one of the two valence categories. AM selection was random, with the restriction that the mean emotional intensity ratings of the to-be-practiced AMs did not differ from those of the unpracticed AMs in that category. Retrieval cues for each AM were printed on separate pages. Retrieval cues were the valence category label (i.e., either "positive memory" or "negative memory") at the top of the page, and the original information in the "where" and "when" units of the original AMs. This information provided a unique cue to ensure that the target AM was indeed retrieved during practice. In addition, the words "who" and "what" were printed at the left margin. This resulted in a set of five pages containing unique retrieval cues for different AMs. The set was presented three times, such that the order of pages was random within each set. This resulted in 15 page RP booklets. Care was taken to ensure that the same AM was not practiced twice in succession (e.g., by being the last $A M$ in one set and the first $A M$ in the consecutive set). Half of the booklets cued positive AMs, the other half of the booklets cued negative AMs.

\section{Distracter phase}

As a distracter parts of Raven's Advanced Progressive Matrices (Raven, 2000) were used.

\section{Test phase}

Category cued recall booklets contained separate pages showing a valence category label at the top and the words "where", "when", "who", and "what" at the left margin. Half of the booklets began with 10 pages referring to the positive category, followed by 10 pages asking for negative memories. This order was reversed for the other half of the booklets.

\section{Procedure}

Participants were tested in groups (maximum of 10 participants). At the outset of session 1, participants were told that they would participate in two unrelated experiments on emotion and memory, with the first looking at autobiographical memory and the second at memory for visual material. The purpose of this cover story was to prevent rehearsal of the AMs in between sessions. Next, participants were instructed to write down 12 positive and 12 negative specific 
memories of personally experienced events in the AM collection booklets. They were told to provide each memory only once and report memories that differed from each other as much as possible. There was no time limit for filling in the booklets.

Session 2 took place three weeks later. Participants were told about the cover story and its purpose. Next they participated in the RP experiment. In the study and RP phases, a beep delivered by a Compaq Laptop computer signaled when participants were to turn the page in their booklet and continue with the next AM. In the study phase, they were instructed to study each page for $15 \mathrm{~s}$. In the RP phase, they were to write down the correct AM in response to the retrieval cues in the RP booklet. Time to respond was 60 s per memory. The duration of the subsequent distracter phase was five minutes. In the final test phase, participants were instructed to fill in the category cued recall booklet by writing down the details of all AMs that they had studied in the initial phase of the RP experiment. There was no time limit for the test phase. Order of testing was counterbalanced such that half of the sample received the booklet that started with the category that was practiced during the RP phase, whereas the other half of the sample started category cued recall with the unpracticed category.

\section{Data scoring}

Two raters (IW \& $\mathrm{BH}$ ) compared testing and study AMs while they were unaware of which valence category and which particular AMs were rehearsed during the RP phase. Information reported in the "when", "where", "who" and "what" units was scored separately. If information given at category cued recall testing matched the information in the study $A M$, that unit was given 1 point. The wording would not have to be identical, as long as the information was similar in essence and no elements were lost or added. A unit received 0.5 point when some information was lost (e.g., 'summer of 1995' instead of 'July 1995') or new elements were added. A unit of information that was not reported or was completely false received 0 points. Thus, scores for each AM ranged between 0 and 4. Next, for each rater, separate scores for AMs that were practiced in the $\mathrm{RP}$ phase $(\mathrm{Rp}+)$, unpracticed AMs from the practiced valence category (Rp-) and $A M s$ from the unpracticed valence category ( $\mathrm{nRp}$ ) were summed and transformed into proportions. In order to determine inter-rater reliability, 
Intraclass Correlation Coefficients (ICCs; Fleiss, 1986) were calculated. ICCs were excellent (i.e., .96, .99, and .97 for Rp+, nRp and Rp-, respectively), and therefore, the data were averaged across raters.

\section{Results}

Retrieval Practice phase

The mean correct recall percentages in the retrieval practice phase were 95.28 $(S D=8.13)$ and $95.69(S D=8.08)$ for "who" and "what" information, respectively. Means for the group with a positive RP phase (who: $M=95.83, S D$ = 8.06; what: $M=95.56, S D=8.34$ ) and the group with a negative RP phase (who: $M=94.72, S D=8.34$; what: $M=95.83, S D=7.82$ ) did not significantly differ, $t(46)=.47$ and $t(46)=.12$ for "who" and "what" information, respectively.

Test phase

Correct recall. Table 3.1 summarizes the relevant means and standard deviations for Rp+, nRp and RP- AMs recalled during the test phase. A 2 (RP Valence: positive vs. negative) × 3 (Retrieval Practice: Rp+ vs. nRp vs. Rp-) Analysis of Variance (ANOVA) with repeated measures on the last factor rendered a significant main effect of Retrieval Practice, multivariate $F(2,45)=$ $34.49, p<.001$. An a priori $t$-test showed that the retrieval practice manipulation was successful in that $\mathrm{Rp}+\mathrm{AMs}$ were better recalled than $\mathrm{nRp} A \mathrm{AMs}, t(47)=$ $7.18, p<.001$, one-tailed. More importantly, a second a priori t-test revealed that correct recall was significantly lower for Rp- than $\mathrm{nRp}$ AMs, $t(48)=1.72, p$ $<.05$, one-tailed. Contrary to expectations, the RP Valence $x$ Retrieval Practice interaction was also significant, $F(2,45)=5.29, p<.01$. In order to break down this interaction, separate ANOVAs were carried out. First, a 2 (RP valence: positive vs. negative) x 2 (Retrieval Practice: $\mathrm{Rp}+\mathrm{vs.} \mathrm{nRp}$ ) analysis rendered a significant Retrieval Practice main effect, multivariate $F(1,46)=57.78, p<$ .001 , and a significant RP Valence $\times$ Retrieval Practice interaction, $F(1,46)=$ $6.74, p<.05$, suggesting a larger practice effect for positive than negative AMs.

Second, a 2 (RP Valence: positive vs. negative) x 2 (Retrieval Practice: nRp vs. $\mathrm{nRp}-$ ) analysis revealed a borderline significant main effect for retrieval practice, multivariate $F(1,46)=3.29, p=.08$, and a significant RP Valence $\times$ Retrieval Practice interaction, $F(1,46)=6.45, p<.05$. Paired $t$-tests revealed that $\mathrm{Rp}+$ 
AMs were recalled significantly better than $n R p$ AMs in both $\mathrm{Rp}$ valence conditions, $t(23)=3.38, p<.01$ and $t(23)=7.60, p<.001$ for negative and positive conditions, respectively. Cued recall of Rp- items was significantly lower than $\mathrm{nRp}$ AMs in the negative valence condition, $t(23)=2.96, p<.01$, but not in the positive valence condition, $t(23)=0.53, p=.60$. Thus, it appears that the RIF-effect was restricted to negative AMs.

Table 3.1. Overall mean correct recall proportions, mean correct recall proportions in Rp valence category and output group in experiment 1 . Standard Deviations are between parentheses.

\begin{tabular}{lccc}
\hline & $\mathbf{R p}+$ & $\mathbf{n R p}$ & $\mathbf{R p -}$ \\
\hline Overall & $.71(.21)$ & $.57(.20)$ & $.54(.22)$ \\
Rp Valence category & & & \\
Positive Rp+ & $.74(.20)$ & $.56(.18)$ & $.57(.20)$ \\
Negative Rp+ & $.68(.22)$ & $.58(.22)$ & $.50(.24)$ \\
Output group & & & \\
Early Rp+ & $.75(.17)$ & $.58(.19)$ & $.57(.21)$ \\
Early Rp- & $.67(.24)$ & $.57(.22)$ & $.50(.23)$ \\
\hline
\end{tabular}

Note. $\mathrm{Rp}=$ Retrieval practice; $\mathrm{Rp}+=$ Memories practiced in the Retrieval Practice Phase; $\mathrm{Rp}-=$ Unpracticed memories that are related to $\mathrm{Rp}+$ memories; $\mathrm{nRp}=$ Unpracticed memories that are unrelated to $\mathrm{Rp}+$ memories.

Valence groups did not significantly differ with regard to $\mathrm{Rp}+\mathrm{AMs}, t(46)=1.13$, $p=.26$, Rp- AMs, $t(46)=1.25, p=.22$, and nRp AMs, $t(46)=0.43, p=.67 . T$ tests showed that the differential RIF effect in the valence groups could not be attributed to differences in emotional intensity ratings ( $t$ 's $>1.09$ and $<0.04)^{5}$.

\section{Output interference}

Next, we explored whether the origins of the RIF effect could be traced back to a non-inhibitory account, such as output interference. This refers to the phenomenon that practiced items have a high probability to be recalled first, and that this interferes with retrieval of (unpracticed) items later in the recall sequence (Anderson, 2003). To explore this, we recorded the position of each $\mathrm{AM}$ in the recall sequence. A $t$-test showed that the mean position of $\mathrm{Rp}$ - AMs $(M=6.04, S D=1.25)$ was indeed significantly higher than that of $\operatorname{Rp}+\mathrm{AMs}(M=$ $4.05, S D=1.03 ; t(47)=6.49, p<.001)$. In order to determine whether initial

5 Emotionality ratings were as follows. In the positive $\mathrm{Rp}+$ group means were $5.40(S D=2.01), 5.70$ $(S D=2.16)$ and $5.34(S D=2.19)$ for $\mathrm{Rp}+, \mathrm{nRp}$ and $\mathrm{Rp}-$, respectively. In the negative $\mathrm{Rp}+$ group means were $5.83(S D=1.32), 5.68(S D=1.63)$ and $5.93(S D=1.43)$ for $\mathrm{Rp}+$, $\mathrm{nRp}$ and Rp-, respectively. 
recall of $\mathrm{Rp}+\mathrm{AMs}$ blocked retrieval of $\mathrm{Rp}-\mathrm{AMs}$, we followed a procedure employed in earlier studies (e.g., Barnier et al., 2004; Macrae \& Roseveare, 2002).

First, we calculated difference scores by subtracting the mean position of Rp+ AMs from the mean position of Rp- AMs. Thus, the higher this difference score, the earlier $\mathrm{Rp}+\mathrm{AMs}$ were recalled relative to $\mathrm{Rp}$ - AMs. We then used this difference score for a median split in each of the two testing order counterbalancing groups (i.e., recall test of Rp AMs first or recall test of $n R p$ AMs first). The halves of these groups with the lowest scores were combined into an early Rp- group, whereas the half of these groups with the higher scores formed an early $\mathrm{Rp}+$ group. Recall data (see table 3.1) were subjected to a 2 (Output Group: early Rp+ vs. early Rp-) x 3 (Retrieval Practice: Rp+ vs. nRp vs. Rp-) ANOVA with repeated measures on the last factor. Neither the Output Group main effect, nor the Output Group x Retrieval Practice interaction reached significance, $F(1,46)=0.85, p=.36$, and $F(2,45)=1.83, p=.17$, respectively. Thus, output order was unlikely to be responsible for the present findings.

\section{Discussion}

All in all, the present results replicate the basic findings of RIF studies that recall of unpracticed related AMs was worse than that of unpracticed unrelated AMs. However, the crucial overall difference between $\mathrm{nRp}$ and Rp- AMs (RIF effect) was small (i.e., $3 \%$ ), especially given that findings with non-idiosyncratic stimuli typically range from $9-20 \%$ (Anderson, 2003). One reason for this small overall effect lies in its interaction with valence: that is, negative but not positive RpAMs were recalled worse than baseline (nRp) AMs. It is important to note that in the negative $\mathrm{Rp}$ condition, the Rp- AMs were negative and the comparison $\mathrm{nRp}$ AMs were positive (and vice versa in the other condition). Thus, the control (nRp) category was always of a different valence than the critical Rp category. It may be argued that negative $\mathrm{nRp}$ AMs provided an inappropriate baseline for their positive Rp counterparts and vice versa. Yet, the finding that baseline (nRp) AMs were comparable with respect to correct recall percentages and emotional intensity ratings speaks against this notion. Nevertheless, the 
question rises whether RIF would be found when baseline and Rp- AMs are of the same valence. In experiment 2 this question was addressed.

\section{Experiment 2}

In experiment 2, the procedure of experiment 1 was modified such that Rp and $\mathrm{nRp}$ categories consisted of AMs of the same valence (i.e., negative). More specifically, in order to obtain sets of AMs that would fit into relatively broad categories, participants retrieved negative AMs in response to two types of cuewords (nouns and adjectives). These combinations of cue-words and AMs were grouped into the categories of "situations" and "traits" for the retrieval practice procedure. During retrieval practice, combinations of category names and cuewords (e.g., trait-sneaky) were used to promote retrieval of the target Rp+ AMs. Expectations were that overall retrieval practice would result in recall differences between the critical $\mathrm{Rp}$ - and baseline (nRp) categories. No effect of Rp category (situations or traits) was expected.

\section{Method}

\section{Participants}

Forty psychology students (16 men) at Maastricht University participated in the study in exchange for course credit. They were all tested at the start of their first year (prior to any courses on memory). None of them had participated in experiment 1.

\section{General overview of procedure}

As in experiment 1, students participated in an AM-collection session and an RP experiment (session 2) consisting of four phases. 


\section{Material}

Similar materials were used as in experiment 1 , with the following modifications.

\section{Session 1 AM collection}

The AM collection booklets contained 20 pages, each with a negatively valenced cue-word printed at the top. Ten cues were nouns and 10 were trait adjectives ${ }^{6}$. Below these cue-words, the words "where", "when", "who", and "what" were printed at the left margin. At the bottom of each page, four $100 \mathrm{~mm}$ Visual Analogue Scales (VASs) asked for judgments of emotional intensity (anchored $0=$ not at all to $100=$ extremely) and valence (anchored $0=$ extremely negative and $100=$ extremely positive). Emotionality and valence was rated for both the time of experiencing the event (retrospective ratings) and the time of recalling it (i.e., in session 1; current ratings).

\section{Study phase}

The booklet contained the 20 AMs that were collected in session 1, now labeled with one of two broad category names: situations (nouns, e.g., situation-theft) and traits (adjectives, e.g., trait - sneaky). The category label was pasted above the cue words in the original AM collection booklets. The VASs were removed.

\section{RP phase}

Combinations of category labels and cue-words were used as retrieval cues for five different AMs. Retrieval cues were printed at the top of each page in the RP booklet, followed by the words "where", "when", "who", and "what" at the left margin. As in experiment 1, the set of five retrieval cues was presented for three times. Half of the booklets cued AMs in the situation category, the other half cued AMs in the trait category. Counterbalancing of cue-words resulted in 20 different versions of the RP booklets (10 for situations and 10 for traits).

\footnotetext{
${ }^{6}$ Nouns were: theft (diefsta), lie (leugen), disappointment (teleurstelling), quarrel (ruzie), accident (ongeluk), heartache (liefdesverdriet), stench (stank), danger (gevaar), blunder (blunder), and loss (verlies). Adjectives were: selfish (egoïstisch), stupid (dom), mean (gierig), impudent (brutaal), indecisive (bes/uiteloos), stubborn (koppig), pessimistic (pessimistisch), sneaky (achterbaks), arrogant (arrogant) and domineering (bazig).
} 


\section{Test phase}

Category cued recall booklets contained category labels at the top of each page followed by the words "where", "when", "who", and "what" at the left margin. There were separate booklets for situations and for traits. Each booklet contained 15 pages so that participants could not infer the maximum number of 10 target AMs by counting the pages. As in experiment 1, order of testing was counterbalanced.

\section{Procedure}

The procedure was similar to that of experiment 1 , with the following modifications. At the start of session 1, participants were instructed to provide specific negative AMs in response to the cue-words in their booklets. Specific memories were defined as pertaining to a specific place and time (within 1 day). Participants were explicitly told that it was okay to report seemingly trivial events, as long as the memories were emotionally negative to them. Each memory was to be reported only once.

In the session 2 study phase, participants were explicitly instructed to study each AM in the context of the corresponding category label and cue-word (e.g., situation - theft) for 30s. In the RP phase, time to respond was $75 \mathrm{~s}$ for the first presentation of the five retrieval cues and 60 and 45 s for the second and third presentations, respectively. The distracter phase was $10 \mathrm{~min}$. Finally, during the test phase, participants were given $15 \mathrm{~min}$ per test booklet (i.e., situations and traits).

\section{Data Scoring}

$\mathrm{Rp}+, \mathrm{Rp}-$ and $\mathrm{nRp}$ memories reported in the cued recall test phase were scored by an independent rater, following the procedure described in experiment 1 . During scoring, it appeared that some memories were reported in the wrong cued recall booklet (i.e., participants reported memories that were originally studied as belonging to the trait category in the situations booklet during recall and vice versa). Therefore, apart from correct recall proportions in the correct category, we also calculated correct recall proportions irrespective of category (i.e., the booklet the memories were reported in). 


\section{Results}

\section{Retrieval practice phase}

Overall, mean recall performance in the retrieval practice phase was 88.83 ( $S D$ $=11.69)$. Groups with a situations RP phase $(M=88.67, S D=12.6)$ and a traits RP phase $(M=89.0, S D=11.03)$ did not differ significantly, $t(38)=0.09, p=$ .93.

\section{Test phase}

Recall in correct category. Table 3.2 summarizes the means and standard deviations of the correct recall proportions for AMs reported in the correct cued recall category and for all AMs that were reported, irrespective of category. Including recall order (practiced category first or non-practiced category first) as a factor in the analyses did not reveal any significant main or interaction effects. Thus, the data were collapsed across this factor in subsequent analyses. A 2 (Rp category: traits vs. situations) x 3 (Retrieval Practice: Rp+ vs. nRp vs. Rp-) ANOVA with repeated measures on the last factor rendered a significant effect of retrieval practice, multivariate $F(2,37)=21.07, p<.001$. A priori t-tests revealed that $\mathrm{Rp}+\mathrm{AMs}$ were significantly better recalled than Nrp AMs, $t(39)=$ 5.56, $p<.001$, one-tailed, and that participants recalled significantly fewer Rpthan $\mathrm{nRp}$ AMs, $t(39)=3.12, p<.01$, one-tailed.

Table 3.2. Mean correct recall proportions in the correct category and irrespective of category in experiment 2. Standard Deviations are between parentheses.

\begin{tabular}{lccc}
\hline & $\mathbf{R p}+$ & $\mathbf{n R p}$ & $\mathbf{R p -}$ \\
\hline Correct category & & & \\
Overall & $.77(.17)$ & $.58(.17)$ & $.47(.24)$ \\
Situations Rp+ & $.79(.18)$ & $.52(.17)$ & $.46(.25)$ \\
Traits Rp+ & $.74(.17)$ & $.63(.15)$ & $.48(.24)$ \\
Irrespective of category & & & \\
Overall & $.78(.14)$ & $.60(.17)$ & $.53(.21)$ \\
Situations Rp+ & $.80(.14)$ & $.54(.17)$ & $.51(.22)$ \\
Traits Rp+ & $.75(.15)$ & $.66(.14)$ & $.56(.20)$ \\
\hline
\end{tabular}

Note. $\mathrm{Rp}=$ Retrieval practice; $\mathrm{Rp}+=$ Memories practiced in the Retrieval Practice Phase; $\mathrm{Rp}-=$ unpracticed memories that are related to $\mathrm{Rp}+$ memories; $\mathrm{nRp}=$ unpracticed memories that are unrelated to $\mathrm{Rp}+$ memories.

Contrary to expectations, the RP category by Retrieval Practice interaction was significant, multivariate $F(2,37)=4.03, p<.05$. Separate ANOVAs were carried out in order to break down this interaction. A 2 (RP category: situations 
vs. traits) $\times 2$ (Retrieval Practice: Rp+ vs. $n R p$ ) ANOVA showed a significant Retrieval Practice main effect, multivariate $F(1,38)=34.94, p<.001$, and a significant RP category $\times$ Retrieval Practice interaction, multivariate $F(1,38)=$ $6.11, p<.05$. Paired $t$-tests revealed that $\mathrm{Rp}+\mathrm{AMs}$ were recalled significantly better than nRp AMs for both the situations, $t(19)=6.23, p<.001$, and traits, $t$ $(19)=2.31, p<.05$, RP groups. Independent $t$-tests showed that groups did not significantly differ regarding $\mathrm{Rp}+\mathrm{AMs}, t(38)=.86$. However, the traits RP group recalled nRp AMs significantly better than the situations RP group, $t(38)=-$ $2.18, p<.05$. A 2 (RP category: situations vs. traits) $\times 2$ (Retrieval Practice: $\mathrm{nRp}$ vs. Rp-) ANOVA showed a significant Retrieval Practice main effect, multivariate $F(1,38)=1.84, p<.001$. The RP category $\times$ Retrieval Practice interaction was not significant, multivariate $F(1,38)=1.84, p=.18$.

Recall irrespective of category. The question rises to what extent the difference between $\mathrm{nRp}$ and Rp- recall performance indicates genuine RIF in the sense that $\mathrm{Rp}$ - AMs became less accessible or available for recall. Therefore, analyses were repeated for correct recall proportions including the AMs that were reported in the wrong category. The 2 (RP category: traits vs. situations) $x$ 3 (Retrieval Practice: Rp+ vs. nRp vs. Rp-) ANOVA with repeated measures on the last yielded a significant main effect of Retrieval Practice, multivariate $F$ (2, $37)=21.78, p<.001$. An a priori t-test showed that the correct recall proportion for $\mathrm{nRp}$ AMs was higher than that of Rp- AMs, $t(39)=2.42, p<.05$, one-tailed. Thus, overall, the relatively poor recall of Rp- AMs cannot be attributed entirely to a greater tendency to recall those AMs in the wrong category.

Again, the RP category $x$ Retrieval Practice interaction was significant, $F(2,37)$ $=5.24, p<.05$. Follow-up analyses showed a similar pattern as the analyses for correct recall proportions in the correct category.

\section{Emotionality and valence}

To check whether the differential recall of $n R p$ AMs in the Rp groups might be attributed to differences in emotionality and valence, session 1 ratings for $\mathrm{nRp}$ 
AMs were subjected to independent $t$-tests ${ }^{7}$. Valence ratings did not differ between RP groups. However, the retrospective emotionality ratings were significantly lower in the situations $\mathrm{Rp}+$ group $(M=50.60, S D=13.03)$ than in the traits $R p+$ group $(M=65.49, S D=13.68), t(38)=-3.53, p<.01$. (Note that $\mathrm{nRp} A M s$ in the situations group were traits $\mathrm{AMs}$ and vice versa). The difference in current emotionality ratings of $\mathrm{nRp} A M s$ was borderline significant $(M=33.75$, $S D=15.83$ and $M=43.30, S D=15.96$ for the traits and situations RP groups, respectively), $t(38)=1.90, p=.07$.

\section{Output interference ${ }^{8}$}

To see whether the RIF effect could be attributed to output interference, the position of each AM in the recall sequence was recorded. A $t$-test showed that the mean position of Rp+AMs $(M=3.57, S D=1.15)$ was lower than that of RpAMs $(M=4.91, S D=1.77), t(37)=3.44, p<.01$. In order to check whether initial recall of $\mathrm{Rp}+\mathrm{AMs}$ hampered later recall of $\mathrm{Rp}$ - AMs, output order preference groups were created following the procedure of experiment 1 , resulting in an early $\mathrm{Rp}+$ group $(n=19)$ and an early $\mathrm{Rp}$ - group $(n=19)$.

Table 3.3. Mean correct recall proportions in the correct category and irrespective of category for output order preference groups in experiment 2. Standard Deviations are between parentheses.

\begin{tabular}{lcccccc}
\hline & \multicolumn{3}{c}{ Early $\mathbf{R p +}(\boldsymbol{n}=\mathbf{1 9})$} & \multicolumn{3}{c}{ Early $\mathbf{R p -}(\boldsymbol{n}=\mathbf{1 9})$} \\
& $\mathbf{R p}+$ & $\mathbf{n R p}$ & $\mathbf{R p}-$ & $\mathbf{R p}+$ & $\mathbf{n R p}$ & $\mathbf{R p}-$ \\
\hline Correct category & $.72(.19)$ & $.56(.15)$ & $.56(.22)$ & $.81(.16)$ & $.62(15)$ & $.43(.21)$ \\
Irrespective of category & $.74(.15)$ & $.60(.14)$ & $.58(.20)$ & $.82(.13)$ & $.64(.15)$ & $.52(.18)$ \\
\hline
\end{tabular}

Note. $\mathrm{Rp}+=$ Memories practiced in the Retrieval Practice Phase; $\mathrm{Rp}-=$ unpracticed memories that are related to $\mathrm{Rp}+$ memories; $\mathrm{nRp}=$ unpracticed memories that are unrelated to $\mathrm{Rp}+$ memories.

Recall data (see Table 3.3) were subjected to a 2 (Output Group: early Rp+ vs. early Rp-) x 3 (Retrieval Practice: Rp+ vs. nRp vs. Rp-) ANOVA with repeated measures on the last factor. Analysis of the correct recall proportion for correctly categorized AMs revealed a significant effect of Retrieval Practice, multivariate $F(2,35)=19.23, p<.001$ and a significant Output Group x Retrieval Practice

\footnotetext{
${ }^{7}$ Mean emotionality and valence ratings were calculated for all $\mathrm{Rp}+, \mathrm{nRp}$ and $\mathrm{Rp}-\mathrm{AMs}$. No other comparisons between the traits and situations Rp groups were significant.

8 Two participants in the situations Rp group did not report Rp- memories at all, leaving 38 participants for this analysis.
} 
interaction, multivariate $F(2,35)=4.45, p<.05$. Contrary to an explanation in terms of output interference, however, the direction of this interaction was such that differences between $\mathrm{nRp}$ and Rp- items were largest in the early Rp- group. In addition, it should be noted that the early Rp- group also recalled significantly more Rp- AMs in the inappropriate $\mathrm{nRp}$ category $(M=.14, S D=.22)$ than the early $\mathrm{Rp}+$ group $\left(M=.01, S D=.05, t_{\text {adjusted DF }}(19.54)=2.43, p<.05\right)$. Therefore the analyses were repeated for correct recall proportions irrespective of category. The Output Group x Retrieval Practice interaction was not significant, multivariate $F(2,35)=1.93, p=.16$. Taken together, output interference does not seem to be responsible for the present results.

\section{Discussion}

All in all, the present findings show RIF in that unpracticed related AMs were more poorly recalled than unpracticed unrelated AMs. The effect was substantially larger (11\% for correctly categorized AMs) than in experiment 1 . These results could be partly traced back to a non-inhibitory phenomenon: some AMs were reported in the incorrect category at cued recall. Nevertheless, after ignoring what category AMs were reported in at recall, the overall difference between $\mathrm{nRp}$ and $\mathrm{Rp}$ - AMs was still a significant $7 \%$.

It should be noted that differences between AMs in the baseline (nRp) categories appeared to be responsible for reducing the magnitude of the overall RIF effect. Baseline AMs in the situations category were rated as more emotional in session 1 and were better recalled in session 2 than traits AMs. These differences were less prominent for $\mathrm{Rp}+$ and $\mathrm{Rp}$ - AMs. Thus, despite efforts to make the Rp categories as similar as possible, the categories may not have provided an appropriate baseline for each other.

\section{General discussion}

The goal of the present studies was to further explore the RIF effect in AM. The results of both experiments show that overall, unpracticed AMs that were related to practiced AMs were more poorly recalled than unpracticed unrelated (baseline) AMs. These findings are in line with earlier studies demonstrating RIF that relied on non-idiosyncratic stimuli (see Levy \& Anderson, 2002). In addition, these findings replicate and extend earlier findings that RIF occurs for AMs 
(Barnier et al., 2004). In the study by Barnier and colleagues (2004), narrow categories of AMs were used (e.g., four AMs in reaction to the cue 'horrified'). The present observation that RIF did occur for relatively broad categories of AMs suggests that the paradigm may contribute to clarifying the mechanisms involved in everyday and perhaps even psychopathological memory phenomena.

Yet, the present findings also suggest that a consequence of modifying the retrieval practice paradigm to more closely resemble real-life is that the magnitude of RIF effects is smaller than in situations that allow for more rigorous experimental control. Anderson (2003) noted that the magnitude of RIF depends on the extent to which the memories that share a retrieval cue compete during retrieval practice. For example, in experiments employing semantic category-exemplar pairs (e.g., Anderson et al., 1994), the effect is larger for strong (i.e., prototypical) exemplars (e.g., Fruit-Orange) than for weak exemplars (e.g., Fruit-Guava). Anderson's (2003) interpretation is that strong exemplars provide more competition at retrieval practice than weak exemplars, rendering more inhibition of the former than the latter. Taxonomic categories have the advantage that they consist of a finite number of members, that differ in the extent to which they are prototypical for that category. Clearly, broad categories of AMs consist of a larger number of members and it is not immediately obvious how to select strong exemplars. Another reason for the relatively small magnitude of the present effects may well lie in systematic differences between the categories that were employed. In both experiments the unpracticed ( $\mathrm{nRp}$ ) baseline AMs belonged to a different category than the practiced AMs. In experiment 2 the baseline (nRp) AMs in the different categories differed in terms of recall performance and emotionality ratings, suggesting that despite similar valence ratings, traits and situations AMs provided a suboptimal baseline for each other. However, in experiment 1 the baseline AMs were comparable on the dimensions of emotionality and recall. Interestingly, the RIF effect occurred for negative AMs, and not for positive AMs. At present, we have no obvious explanation for this finding. Future research may further clarify the role of valence in RIF.

On a more theoretical note, the question rises what mechanism underlies RIF effects. One dominant view in the literature on RIF (e.g., Anderson, 2003; Levy 
\& Anderson, 2002) is that the long-term memory representations of unpracticed related exemplars are (temporarily) inhibited in the sense that activation levels are decreased. However, since inhibition is difficult to measure directly, alternative non-inhibitory accounts have been put forward (e.g., MacLeod, Dodd, Sheard, Wilson, \& Bibi, 2003). One such possibility is output interference, referring to the phenomenon that practiced items have a high probability to be recalled first, and that this interferes with retrieval of (unpracticed) items later in the recall sequence (Anderson, 2003). The results showed that in the present experiments, output interference was an unlikely alternative explanation. Yet, that does not mean that non-inhibitory processes can be ruled out altogether.

The present experiments were inspired by the notion that RIF (or even inhibition) might play a role in the clinical situation in which intrusive memories (IMs) and OGMs co-occur. How does this idea compare to other explanations regarding the origins of OGM? One influential account is the affect regulation hypothesis (Williams, 1996; see also Raes, Hermans, de Decker, Eelen, \& Williams, 2003) that holds that the retrieval of categoric AMs would serve a protective function, in that the sharp affect that accompanies retrieval of specific (traumatic) memories is avoided. Such an account does not readily accommodate findings that IMs and OGMs are linked. For example, if categoric retrieval mainly serves a protective function, why would it fail for exactly those AMs that are most distressing in those who suffer from them? An advantage of the present idea is that its starting point lies in what is known about normal memory phenomena. In this sense, OGM is viewed as an (unwanted) byproduct of the repetitive execution of the retrieval process that characterizes IM. Perhaps retrieving the same set of AMs over and over again results in an ongoing state of reduced accessibility or availability of closely related AMs. Such impairment might spread to more distant AMs.

Taken together, the retrieval practice paradigm may provide a starting point for providing an experimental analogue for the clinical situation in which OGM is correlated with IM. Yet, several issues need to be addressed in future studies before it can be inferred that RIF indeed plays a role in that situation. To begin with, a limitation of the present method is that practiced (Rp+) AMs do not closely resemble the characteristics of IM. The retrieval practice procedure consists of repeatedly retrieving several (five in these experiments) different 
AMs in an intentional manner, triggered by abstract verbal cues. By contrast, clinical research (see Ehlers, Hackmann, \& Michael, 2004) suggests that patients with Posttraumatic Stress Disorder (PTSD, in which IM is a hallmark symptom, American Psychiatric Association, 1994) experience only a few different intrusions (i.e., two on average; Hackmann, Ehlers, Speckens, \& Clark, 2004). In addition, a prominent feature of IMs is that they are perceived as uncontrollable and that their retrieval is involuntary rather than intentional frequently brought about by sensory cues. It would be interesting to see if inducing involuntary recall of only one AM (e.g., through thought suppression, Wegner, 1989) hampers specificity of other AMs. Furthermore, it remains to be seen how the RIF-effect compares to the relative inability to be specific as in the clinical phenomenon of overgeneral autobiographical memory. Especially in the case of experiment 2, there were indications that retrieval practice affected the association between cue word and AM rather than the ability to retrieve the target AM itself. That is, some of the memories in that experiment were recalled in the incorrect category, indicating that participants forgot the source of those AMs rather than the AMs themselves. In that respect, the situations and traits labels in experiment 2 were rather artificial. Future research may concentrate on employing AMs that naturally fall into obvious categories (such as positive and negative memories). A related problem that needs to be addressed is that the present method does not take discrepancies in recall of details between sessions into account. That is, if an error is made in session 1, this would constitute the study material for the retrieval practice experiment, irrespective of whether the correct detail is recalled at the time of study. Providing participants with the opportunity to correct their AMs at time of study may solve this problem. Finally, the present experiments involved healthy undergraduate participants, limiting the generalizability of the results. Future studies should explore whether RIF of AMs occurs in different samples. All in all, the conceptualization of OGM as the result of processes involved in $\mathrm{IM}$ raises interesting possibilities. Addressing these issues is a challenge for future research. 


\title{
Effects of repeated retrieval of central and peripheral details in complex emotional slides
}

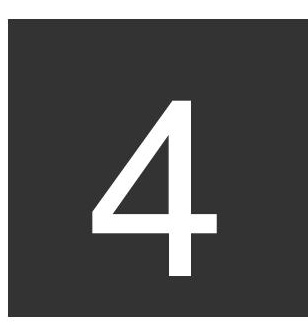

\author{
B.J.A. Hauer, I. Wessel, H. Merckelbach, A. Roefs, T. Dalgleish
}

Adapted from: Memory (2007: 15, 435-449)

Research has demonstrated that repeated retrieval enhances memory for practiced verbal information, but undermines correct recall of unpracticed related verbal information, a phenomenon known as retrieval-induced forgetting (RIF). This paper addresses the question of what happens with memory for unrehearsed aspects of an emotional picture when retrieval of other aspects of that picture is practiced. We investigated in three experiments whether repeated retrieval of certain details of negative emotional slides undermines recall of unrehearsed details of such slides. Experiments 1 and 3 showed that repeated retrieval of central details enhanced memory for these details. However, correct recall was not impaired for the unrehearsed central and peripheral details, although more commission errors were produced. In experiment 2, retrieval of peripheral details was practiced. Recall for peripheral details was enhanced but again correct recall of unpracticed (central) details was not undermined and this time there was no increase in commission errors. This failure to find RIF effects for complex visual material is at odds with the RIF literature for verbal material, and potential reasons for this are discussed. The data also extend previous studies in demonstrating that extensive retrieval can increase the number of commission errors. 


\section{Introduction}

Emotional events are strongly encoded in memory (e.g., Cahill, Gorski, \& Le, 2003; Cahill \& McGaugh, 1998). Emotional memories also tend to be frequently rehearsed over time (e.g., Conway et al., 1994; Finkenauer et al., 1998). However, it is plausible to assume that not all aspects of an emotional event are rehearsed equally well. For instance, people with Posttraumatic Stress Disorder (PTSD) persistently re-experience some parts of a traumatic event in the form of intrusive memories (American Psychiatric Association [APA], 1994; Brewin, 1998). In contrast, they might be unable to recall other aspects of that same event, a phenomenon that some researchers have related to dissociative amnesia (e.g., Holmes et al., 2005; see also APA, 1994). Another relevant example is eyewitness testimony. Police interviewers will often focus on particular key details of a crime but, what happens with eyewitness memory for other, unrehearsed details? There is some experimental evidence to suggest that memory for these aspects deteriorates as a result of rehearsal of the key details (e.g., Wright, Loftus, \& Hall, 2001).

Relevant to the issue of rehearsal effects are studies using Anderson and colleagues' retrieval practice paradigm (e.g., Anderson, Bjork, \& Bjork, 1994; Anderson \& Spellman, 1995). This paradigm offers a way to investigate the impact of repeated retrieval of particular information on recall of unrehearsed information. The paradigm typically consists of three phases and relies on word stimuli (e.g., Anderson et al., 1994). During a study phase, participants learn a number of category-exemplar associations (e.g., Fruit-Banana; Fruit-Orange; Garden-Tree). Subsequently, participants are directed to retrieve half of the studied exemplars from half of the categories by completing category-stem pairs (e.g., Fruit-Ba__). In this retrieval practice phase, the critical exemplars are practiced three times. After a distracter phase, participants engage in a cued recall test. They are cued with a category name (e.g., Fruit, Garden) to recall all of the exemplars from all of the categories that were presented in the study phase. There are thus three types of exemplars: practiced exemplars (FruitBanana), unpracticed related exemplars (Fruit-Orange), and unpracticed unrelated exemplars (Garden-Tree). Typically, recall for practiced exemplars (Fruit-Banana) is enhanced. This is called the retrieval practice effect. The more 
interesting result is that fewer unpracticed related exemplars (Fruit-Orange) than unpracticed unrelated exemplars (Garden-Tree) are recalled. This outcome is dubbed retrieval-induced forgetting (Anderson et al., 1994). According to Anderson et al. (1994), this temporary forgetting is caused by inhibition of competing exemplars that are associated with the same (retrieval) cue as the retrieval-practiced exemplars (but see MacLeod, Dodd, Sheard, Wilson, \& Bibi, 2003). The retrieval-induced forgetting effect has been replicated with a variety of stimulus material (for a review see Levy \& Anderson, 2002), including words (e.g., Anderson \& Spellman, 1995), visuo-spatial objects (Ciranni \& Shimamura, 1999), and personality traits (Macrae \& MacLeod, 1999).

The question arises as to whether a similar pattern occurs with more complex and emotional situations in real life. Thus, for example, one could speculate that dissociative amnesia for particular aspects of a traumatic event is a consequence of (persistent) repeated retrieval of certain other aspects of the same traumatic events in the form of intrusive memories (see also Brewin \& Andrews, 1998). Similarly, repetitive police interviews about particular aspects of a serious crime may result in forgetting other details related to that same offence. Indeed, as indicated above, several studies (MacLeod, 2002; Saunders \& MacLeod, 2002; Shaw, Bjork, \& Handal, 1995) have demonstrated that repeated questioning enhances participants' memory for the rehearsed aspects, but promotes forgetting of the unrepeated details. Additionally, some studies have demonstrated retrieval-induced forgetting with emotional material (e.g., Amir, Coles, Brigidi, \& Foa 2001; Barnier, Hung, \& Conway, 2004). For example, two studies (Barnier et al., 2004; Wessel \& Hauer, 2006) showed that retrieval practice of emotional autobiographical memories impaired recall of unpracticed autobiographical memories that were related to the same cue. Taken together, these results do not only imply that retrieval-induced forgetting can be generalized to emotional material, but also to more complex material than, for example, words.

So far, we have restricted our discussion to the effect of repeated retrieval on subsequent recall of unrehearsed information. However, apart from retrieval, encoding might also be responsible for differential recall. Research has demonstrated that not all aspects of emotional stimuli are encoded equally well (e.g., Christianson, 1992; Heuer \& Reisberg, 1990). For example, several 
studies (Christianson \& Loftus, 1987; 1991) compared memory for emotional and neutral slides revealing that central details (i.e., information reflecting the essence) of emotional slides are better retained than those of neutral slides. In many studies, the opposite is found for peripheral details (i.e., thematically irrelevant information), with memory of peripheral details being poorer for emotional than for neutral material (but see Wessel, van der Kooy, \& Merckelbach, 2000, for boundary conditions). According to the attentional narrowing hypothesis (see Christianson, 1992), the physiological arousal that accompanies experiencing negative emotional events results in devoting more attention to central details than to peripheral details. Consequently, superior encoding of central details enhances memory for these details, but undermines memory for peripheral details. This possibly also makes peripheral details more prone to commission errors (i.e., adding or distorting details, e.g., Candel, Merckelbach, \& Zandbergen, 2003; Heuer \& Reisberg, 1990). For example, Heuer and Reisberg (1990) noted that most commission errors occur for poorly encoded details (i.e., peripheral aspects) and that such errors are consistent with pre-existent cognitive schemata of emotional situations.

The present experiments investigated whether the typical pattern of encoding effects in emotional material becomes even more pronounced when central and peripheral details are subjected to rehearsal. This issue was addressed in three experiments. Inspired by the retrieval practice literature, we used a repeated retrieval method with negative emotional slides to investigate the effect of repeated retrieval of either central (experiment 1 and 3 ) or peripheral (experiment 2) details on the recall of unrehearsed central and peripheral details. Whereas previous studies using complex emotional material focused on complete memories (e.g., Barnier et al., 2004; Wessel \& Hauer, 2006), the present experiments focus on different types of details of complex visual scenes.

\section{Experiment 1}

Experiment 1 explored whether repeated retrieval of central aspects of emotional slides would radicalize the pattern typically observed in studies on central and peripheral detail memory, i.e. good recall of central details and poor recall of peripheral details (Christianson, 1992; see also Reisberg \& Heuer, 
2004). The main research question was: Does repeated retrieval of central details undermine memory for peripheral details? Following Christianson (1992), central details were defined as all details that reflect the gist of the visual scene (i.e., thematically essential to the slide). Details that were not relevant to the essential theme (i.e., that can be left out without changing the gist of the visual scene) were counted as peripheral details. A between-subjects design was employed (compare Shaw et al., 1995), in which the experimental condition involved repeated retrieval of central details. In contrast, the control condition involved an unrelated memory task.

We hypothesized that the retrieval practice group would show a retrieval practice effect (i.e., enhanced correct recall of central details) compared to the control condition (e.g., Anderson et al., 1994; Shaw et al., 1995). We further anticipated that peripheral detail memory would be affected in two ways. First, in line with the retrieval-induced forgetting literature (e.g., Anderson et al., 1994), we expected that repeated retrieval of central details would reduce the number of correctly recalled peripheral details in the retrieval practice group relative to the control group. Second, previous studies found that repeated retrieval of central details compromises the accuracy of peripheral details in the form of increased commission errors (i.e., adding or distorting details) for such details (Heuer \& Reisberg, 1990) and that unrehearsed details become more susceptible to misinformation (Saunders \& MacLeod, 2002). We, therefore, hypothesized that repeated retrieval of central details would induce more commission errors in reports of peripheral details relative to the control condition.

\section{Method}

\section{Participants}

Seventy first-year undergraduate psychology students (14 men) from Maastricht University participated in the current study. Mean age was 19.8 years (Range: 18-26). Participants were randomly assigned to either a retrieval practice or a control condition, resulting in 35 participants in each condition. The groups did not differ in mean age, $t(68)=.75, p=.45$, nor in sex ratio, $\chi^{2}(1)=.35, p=.55$. Participants received either course credit or $€ 7.50$ for their participation. The 
experiment was approved by the standing Ethical Committee of the faculty of psychology of Maastricht University.

\section{Stimulus material}

The stimulus material consisted of five negative emotional slides and one practice slide. All of the slides were selected from the International Affective Picture System (IAPS; Lang, Bradley, \& Cuthbert, 1998). These slides were selected on the basis of two criteria. Firstly, slides had a negative valence (IAPS score below 3 on a 9-point scale) with high arousal (IAPS score above 5 on a 9point scale). Secondly, in a pilot study $(N=8)$, participants were asked to examine 14 slides and to recall as many of the details as possible after having seen the slides for $5 \mathrm{~s}$. The rationale was to determine the maximum number of details that could be recalled from the slides. Participants were also provided with the definitions of central and peripheral details presented above and were then asked to rate the details that they recalled on a 4-point scale $(1=$ 'highly peripheral' to 4 = 'highly central"). On the basis of this categorization, details were assigned to the central and peripheral categories.

Based on the pilot study, the five slides (IAPS numbers were 3350, 3530, 9250, 9253, 9433) were selected that contained sufficient and similar numbers of central and peripheral details. This was done to ensure that participants would have enough opportunity to encode peripheral details and to minimize possible floor effects. The total number of details in a category was the total of all details that were named by at least one of the pilot participants. The total number of details reflecting central objects of the slides was 66 . There were some central details that did not reflect the central object, but that were nevertheless critical to the gist of the scene (e.g., the facial profile of a victim, where the attacker is the central object). The number of these details was 26. The total number of peripheral details was 57 . The total number of details thus reflected a complete and accurate description of the details present in the slides. See figure 4.1 for an example of the different detail categories in the slides.

Furthermore, each slide was given a title to function as a category label. The titles of the experimental slides were: Sick baby (3350), Threat (3530), Social worker (9250), Injured woman (9253), and Wounded man (9433). Torture (9252) was the name of the example slide. 
Threat

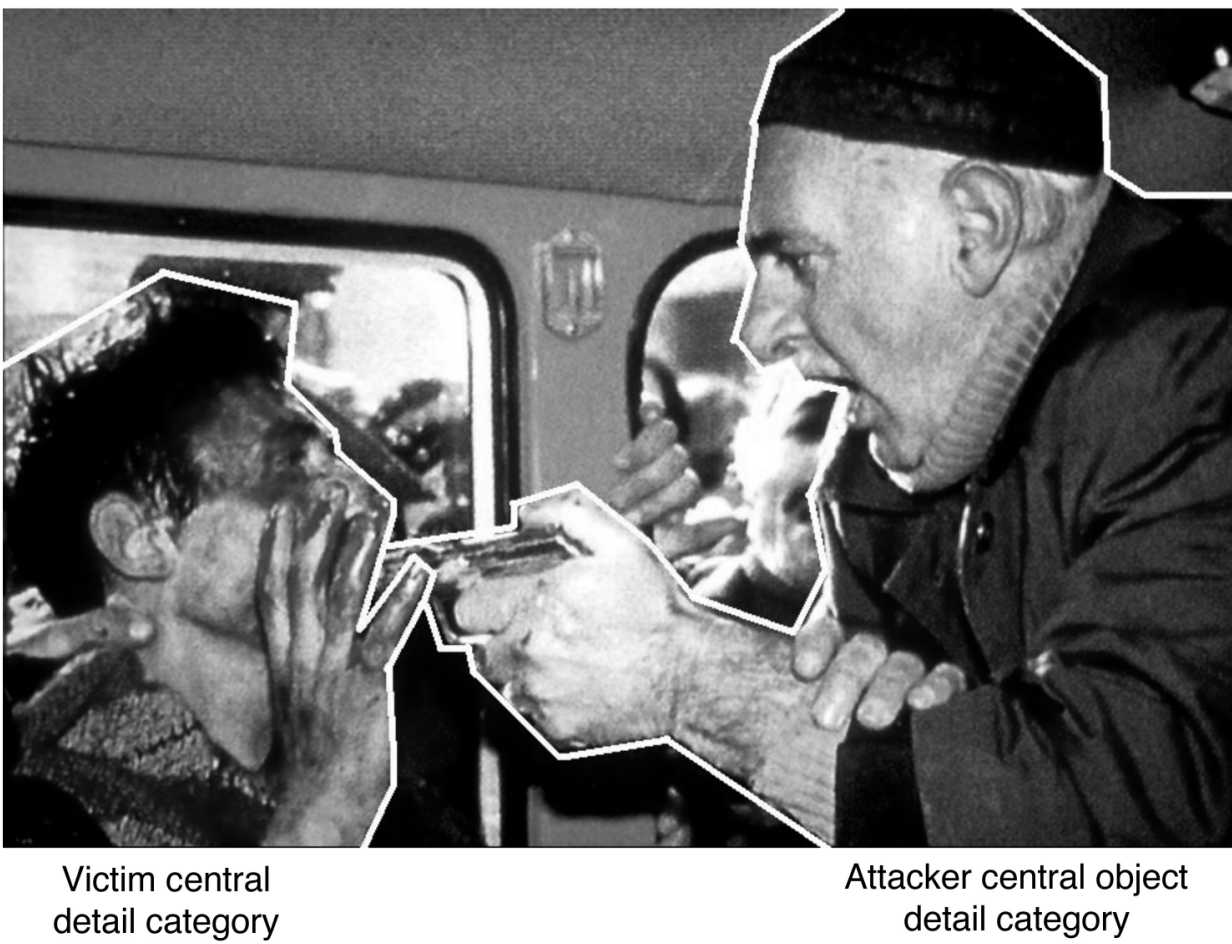

Figure 4.1. Stimulus material: Illustration of the type of details in the categories that participants were asked to remember. On the right hand side, the white line around the 'attacker' indicates which details were included in the central object detail category. On the left hand side, the white line around the 'victim' indicates which details belong to the central detail category. All of the other details were in the peripheral detail category.

\section{Retrieval practice booklets}

Participants in the retrieval practice condition received 15 page retrieval practice booklets. On separate pages, the slide title and the retrieval practice question for each individual slide was presented. For each slide three standardized retrieval practice question were formulated about the central object, for instance 'Describe the attacker in as much detail as you can' for the slide Threat. The experimenter wrote down all of the details that the participants recalled aloud in the retrieval practice booklets. Participants were encouraged to report all of the details of the central object (e.g., the attacker) that they could remember. When participants had difficulty describing any more essential details following the first open question, the experimenter asked two additional open questions to 
encourage retrieval practice (e.g., 'What clothes is he wearing?' and 'What is his position?'). This was to ensure, as far as possible, that all participants recalled the same details during the retrieval practice phase. Care was taken that participants did not elaborate further on the other unpracticed central (e.g., the victim) and unpracticed peripheral details. In pseudo-random order, each slide was rehearsed three times by presenting the same retrieval practice questions. The same slide was never practiced twice in direct succession.

\section{Control condition}

As an alternative to the retrieval practice phase, participants in the control condition engaged in an unrelated memory task (Autobiographical Memory Test, AMT; Williams \& Broadbent, 1986) and filled in some questionnaires.

\section{Distracter task}

This was a non-verbal intelligence task in both conditions (Raven's Advanced Progressive Matrices; Raven, 2000).

\section{Test booklets}

These booklets contained five pages. Each page presented the slide-title (e.g., Threat) in order to cue recall. The order of the slides was random and differed for each participant. All participants from both conditions were asked to recall aloud all of the details that they could remember. The experimenter wrote down in the test booklets everything that the participants said.

\section{Procedure}

Participants were tested individually. In the laboratory, all participants heard a cover story telling them that they were to do two different experiments. They were told that the first experiment involved recording of physiological responses while watching a series of negative emotional slides. Thus, the study phase was incidental. This was done to enhance ecological validity and to reduce the probability of elaborative encoding and thus integration between to-be-practiced and to-be-unpracticed details during the study phase (e.g., Anderson \& McCulloch, 1999). Participants were told that the second experiment involved the validation of a non-verbal intelligence task. Nothing was said about the 
upcoming surprise cued recall task. Participants subsequently signed a consent form and electrodes were attached to their hands.

Participants sat approximately $1 \mathrm{~m}$ in front of a $66 \times 91 \mathrm{~cm}$ Dell monitor on which full screen versions of the slides were shown. During the slide show (study phase), the lights were switched off to enhance emotional intensity. To begin, an example slide was shown, followed by the five critical slides in random order. Each trial consisted of the presentation of slide titles for $1.5 \mathrm{~s}$, followed by presentation of the slides for $5 \mathrm{~s}$. Intertrial intervals were $6 \mathrm{~s}$. After watching the slide show, electrodes were removed. Next, participants in the retrieval practice condition immediately worked through the retrieval practice booklet. This retrieval practice phase took $20 \mathrm{~min}$. Participants were first instructed about the retrieval practice procedure and they practiced with the example slide. Next, using the retrieval practice booklets, the experimenter interviewed these participants about all of the details of the central objects. Participants got $60 \mathrm{~s}$ to answer each retrieval practice question and to recall as many central object details as they could remember. Control participants engaged in the unrelated memory task (the AMT). Subsequently, all participants took part in the $10 \mathrm{~min}$ distracter task (Raven's Matrices).

Finally, all participants engaged in the cued recall test phase. With the slide titles as cues, participants were instructed to verbally recall all of the central and peripheral details of the slides presented during the study phase. Participants were encouraged to report everything they could remember even if they thought it would be of no importance. After the test phase, participants were debriefed and thanked for their participation.

\section{Scoring}

Two types of dependent measures were scored: correctly recalled details and commission errors. Scoring was based on a detailed scoring protocol, containing extensive descriptions of correct details in three categories: central object details, central details, and peripheral details. A detail was defined as a piece of information that was visually present on the slide based on the pilot data described above. Each correctly reported detail received one point. For example, the correctly recalled phrase 'red t-shirt with white lettering' received four points, whereas only recalling 'red t-shirt' was scored with two points. 
Commission errors were defined as adding new information or distorting presented information. An example of adding new information is recalling 'a house' that was not visually present on the slide. An example of distorting presented information is recalling a 'blue t-shirt' or 'red jacket' instead of 'red tshirt'. Each piece of added or distorted information received one point.

All cued recall protocols were scored by an independent rater who was blind to the experimental conditions. A second rater $(\mathrm{BH})$, who was also blind to the experimental conditions, scored all of the free recall reports of 20 randomly selected participants (29\% of the sample): 10 reports even from the retrieval practice and control conditions. Intraclass Correlation Coefficients (ICC; see Fleiss, 1986) were computed. Interrater agreement was high. For correctly reported details, we found ICCs of .90 for the total number of recalled details, .92 for central details, and .86 for peripheral details. ICCs for commission errors were .85 for total number of commissions, .78 for commissions in the central details, and .85 for commissions in peripheral details.

\section{Results and discussion}

Where relevant, raw data were converted into proportions based on the total number of details in the visual scene (see above).

The mean proportion of correctly recalled practiced central object details in the retrieval practice phase for the retrieval practice group was $41 \%(S D=12.32)$. The mean numbers of reported details that properly belonged to the unpracticed central and unpracticed peripheral details during the retrieval practice phase was $1.34(S D=1.57)$. The mean number of reported commission errors during the retrieval practice phase was $6.13(S D=2.70)$.

Table 4.1 presents the relevant means and standard errors for the proportions of correctly recalled details and the number of commission errors in the different detail categories in the test phase. A 2 Retrieval Practice (yes vs. no) $\times 3$ Detail (practiced central object vs. unpracticed central vs. unpracticed peripheral) mixed ANOVA demonstrated a significant main effect of detail, $F(2,68)=$ $123.41, p<.001$. Follow up paired samples $t$-tests demonstrated that practiced central object details were significantly better recalled than unpracticed central details, $t(69)=4.25, p<.001$, and than unpracticed peripheral details, $t(69)=$ 
19.55, $p<.001$. Unpracticed central details were also better recalled than unpracticed peripheral details, $t(69)=9.59, p<.001$. There was no main effect of retrieval practice, $F(2,68)=1.52, p=.22$, neither was there a Detail $\mathrm{x}$ Retrieval Practice interaction, $F(2,68)<1$.

Regarding the commission errors, a different pattern of results emerged. We did not calculate proportions for commission errors because there was no absolute total number of commissions that could serve as a reference. The 2 Retrieval Practice (yes vs. no) $\times 3$ Detail (practiced central object vs. unpracticed central vs. unpracticed peripheral) mixed ANOVA revealed main effects for detail, $F$ (2, $68)=34.65, p<.001$, and for retrieval practice, $F(2,68)=10.98, p=.001$. These main effects were qualified by a significant Detail $x$ Retrieval Practice interaction, $F(2,68)=4.18, p=.02$. Follow-up independent samples $t$-tests demonstrated that the retrieval practice group produced significantly more commission errors in the peripheral details, $t(68)=3.72, p<.001$, tended to produce more commission errors in the unpracticed central details, $t(68)=$ $1.85, p=.07$, but did not produce more commission errors in the practiced central object details, $t(68)=1.48, p=.14$, relative to controls.

Table 4.1. Mean proportion of correctly recalled details and number of commission errors in the test phase (standard errors are within parentheses) of retrieval practice $(n=35)$ and control $(n=35)$ groups.

\begin{tabular}{lccc}
\hline & $\begin{array}{l}\text { Practiced central } \\
\text { object details }\end{array}$ & $\begin{array}{l}\text { Unpracticed central } \\
\text { details }\end{array}$ & $\begin{array}{l}\text { Unpracticed } \\
\text { peripheral details }\end{array}$ \\
\hline $\begin{array}{l}\text { Correctly recalled } \\
\text { details }\end{array}$ & & & \\
$\begin{array}{l}\text { Practice group } \\
\text { Control group }\end{array}$ & $.45(.02)$ & $.37(.04)$ & $.14(.01)$ \\
$\begin{array}{l}\text { Commission } \\
\text { errors }\end{array}$ & $.41(.02)$ & $.33(.04)$ & $.13(.01)$ \\
$\begin{array}{l}\text { Practice group } \\
\text { Control group }\end{array}$ & $5.71(.45)$ & & \\
\hline
\end{tabular}

The present study did not find a significant retrieval practice effect for recall of practiced central details across groups. Furthermore, there was no retrievalinduced forgetting as repeated retrieval of central object details did not lower correct recall of unpracticed central and unpracticed peripheral details, relative to the control condition. These data are at odds with findings from earlier studies employing a retrieval practice paradigm (e.g., Anderson et al., 1994; MacLeod, 2002; Saunders \& MacLeod, 2002). However, repeated retrieval of thematically essential details was found to induce more commission errors in the unpracticed 
peripheral details and thus replicates earlier findings that repeated retrieval of central details elicits more commission errors in peripheral details (e.g., Heuer \& Reisberg, 1992). The current results are also consistent with other literature showing that extensive retrieval enhances the tendency to make commission errors (e.g., Henkel, 2004; Schwartz, Fisher, \& Hebert, 1998).

The most likely explanation for the lack of a retrieval practice effect in the present data is that the retrieval practice success rate was rather low $(41 \%$ correct recall). A possible explanation for this figure is that the calculated proportions were based on all details in the central object detail category that were named by at least one participant in our pilot study. Because there was considerable variation in the details that participants recalled, the total number of details was somewhat large and consequently this may have resulted in relatively low proportions for any given participant. Because of the lack of a significant retrieval practice effect, it is difficult to interpret why the retrievalinduced forgetting effect did not emerge. One might argue that there was not enough retrieval practice to inhibit unpracticed information and, therefore, there was no opportunity to obtain the retrieval-induced forgetting effect. Thus, there is a need for a further study in which a significant retrieval practice effect is obtained and in which the presence or absence of retrieval-induced forgetting can be ascertained.

There some methodological points worth highlighting. First, it is interesting to note that the number of reported commission errors during the retrieval practice phase was relatively high. This may be related to the interviewing procedure, in which participants were asked to try and recall as many (correct) details as they could. Consequently, participants may have recalled information when they were not sure whether it was correct, instead of saying nothing. Second, we do not know precisely what happened during the retrieval practice phase when participants were endeavoring to remember central object details. They may have connected these central object details to the unpracticed central and peripheral details that should have been ignored during retrieval practice. This spontaneous connection may have resulted in integration among the practiced and unpracticed details, which may have mitigated possible retrieval-induced forgetting effects (see Anderson \& McCulloch, 1999). 
Third, retrieval-induced forgetting may not have emerged because unpracticed peripheral details were weak competitors of practiced central object details (see Anderson, 2003). Earlier studies (Anderson et al., 1994; Bäuml, 1998) have shown that retrieval-induced forgetting is more pronounced when unpracticed related items are strong rather than weak exemplars of a semantic category. The idea (see Anderson, 2003) is that strong exemplars have a high probability of being accessed during a retrieval practice phase, even if they are not explicitly practiced. Competition between strongly associated target and nontarget exemplars during retrieval practice, it is argued, therefore needs to be resolved by suppression of the non-target exemplars. Subsequently, cued recall of these putatively suppressed non-target exemplars should be relatively poor, as seems to be the case (Anderson et al., 1994; Bäuml, 1998). In experiment 1, central details were thematically essential for the slide and strongly related to the retrieval cue. Peripheral details, on the other hand, were by definition thematically unimportant and may therefore have been only weakly associated with the title cues. This may have resulted in weak or even no competition between central and peripheral details during retrieval practice. Thus, there may have been no need for suppression of the peripheral aspects and hence no retrieval-induced competing effects (see Anderson, 2003).

\section{Experiment 2}

Experiment 2 was designed to address some of the methodological issues raised by experiment 1 . The first goal was to increase competition between practiced and unpracticed details by instructing participants to repeatedly retrieve the peripheral details leaving the central aspects as highly competitive unpracticed details. We also speculated that the relatively long intertrial stimulus time of $6 \mathrm{~s}$ might have caused elaboration of the to-be-ignored unpracticed central and peripheral details during the study phase in experiment 1 . We therefore reduced the intertrial stimulus time in experiment 2. We furthermore adjusted the retrieval practice procedure so that participants were asked more directed retrieval questions, instead of the interview format used in experiment 1. We anticipated that this would increase the number of correctly recalled practiced peripheral details (i.e. a higher success rate) and lower the number of commission errors for the peripheral details during retrieval practice. We also 
expected that there would be less opportunity to integrate the practiced peripheral and unpracticed central (object) details during the retrieval practice phase.

In terms of hypotheses for experiment 2, based on the retrieval-induced forgetting literature (e.g., Anderson et al., 1994), we predicted that retrieval practice of peripheral aspects would reduce correct recall of central details because competition was increased (see Anderson, 2003), relative to a no retrieval practice control condition. We also expected a retrieval practice effect in that participants who had engaged in retrieval practice would recall more practiced peripheral details than control participants. Finally, to the extent that commission errors for peripheral details in experiment 1 were a function of the retrieval manipulation (e.g., Henkel, 2004), we now anticipated more commission errors for unpracticed central (object) details in experiment 2.

\section{Method}

\section{Participants}

Fifty-nine second-year psychology students (20 men) from Maastricht University participated in this study. Mean age was 22.16 years (Range: 20-29). Participants were randomly assigned to one of two testing conditions, resulting in 30 participants in the control condition and 29 participants in the retrieval practice condition. The groups did not differ in mean age, $t(57)=1.64, p=.11$, nor in sex ratio, $\chi^{2}(1)=1.00, p=.32$. All participants received course credit for their participation.

\section{Materials}

The stimulus material, test booklets, distracter tasks and apparatus were exactly the same as in experiment 1.

\section{Retrieval practice booklets}

These contained retrieval practice questions about the peripheral details. Following the procedure of Shaw et al. (1995), three different types of retrieval practice questions were constructed. The first retrieval practice episode contained three true-false questions about each slide (for example, 'Were there any bystanders on the slide?'). During the second retrieval episode, more 
specific questions were asked about the location of the peripheral details (for example, 'Where were the bystanders standing?'). The third retrieval episode contained questions like 'Describe the appearance of the bystanders'. Thus, the questions asked for detailed descriptions of the peripheral details.

\section{Control condition}

As an alternative to the retrieval practice phase, participants in the control condition already started with the Raven task (Raven's Progressive Matrices; Raven, 2000).

\section{Procedure}

The procedure was basically the same as for experiment 1, with three modifications. To begin with, participants heard a different cover story. They were told that they were to take part in an experiment testing the impact of negative emotion on performance on a non-verbal intelligence task. Secondly, the presentation of the test slides was set at $7 \mathrm{~s}$. This was done to ensure that the peripheral details would be sufficiently well encoded to promote successful retrieval practice. Third, intertrial intervals were now set at $2 \mathrm{~s}$.

\section{Scoring}

Cued recall data were scored by the same independent rater as in experiment 1 , who remained blind to condition membership.

\section{Results and discussion}

For participants in the retrieval practice condition, the mean proportion of correctly recalled details in the retrieval practice phase was $34 \%(S D=7.92)$. The mean number of reported unpracticed central object and central details during the retrieval practice phase was .48 $(S D=.54)$. The mean number of reported commission errors during the retrieval practice phase was 2.11 ( $S D=$ 1.13).

Table 4.2 presents relevant means and standard errors for the proportions of correctly recalled details and the numbers of commission errors in the different detail categories in the test phase. All proportions were based on the total number of details as described in the method section of experiment 1 . The 2 Retrieval Practice (yes vs. no) x 3 Detail (unpracticed central object vs. 
unpracticed central vs. practiced peripheral) mixed ANOVA yielded a main effect for detail, $F(2,57)=95.93, p<.001$. There was no main effect for retrieval practice, $F(2,57)<1$. However, there was a significant Detail $\mathbf{x}$ Retrieval Practice interaction, $F(2,57)=3.86, p=.02$. Follow-up independent samples $t$-tests demonstrated that the retrieval practice group significantly recalled more of the practiced peripheral details compared with controls, $t(59)=$ $3.11, p=.003$, showing a retrieval practice effect. There were no significant differences between groups in the correct recall of unpracticed central details, $t$ (59) $<1, p=.43$, or unpracticed central object details, $t(59)<1, p=.85$.

Table 4.2. Mean proportion of correctly recalled details and number of commission errors in the test phase (standard errors are within parentheses) of the retrieval practice $(n=29)$ and control $(n=30)$ groups.

\begin{tabular}{lccc}
\hline & $\begin{array}{l}\text { Unpracticed central } \\
\text { object details }\end{array}$ & $\begin{array}{l}\text { Unpracticed } \\
\text { central details }\end{array}$ & $\begin{array}{l}\text { Practiced } \\
\text { peripheral details }\end{array}$ \\
\hline $\begin{array}{l}\text { Correctly recalled } \\
\text { details }\end{array}$ & & & \\
$\begin{array}{l}\text { Practice group } \\
\text { Control group }\end{array}$ & $.53(.03)$ & $.43(.03)$ & $.27(.02)$ \\
Commission & $.53(.03)$ & $.46(.03)$ & $.18(.02)$ \\
errors & & & \\
Practice group & $4.07(.50)$ & $2.59(.32)$ & $4.97(.71)$ \\
Control group & $4.97(.49)$ & $2.07(.32)$ & $3.90(.69)$ \\
\hline
\end{tabular}

In terms of commission errors, the 2 Retrieval Practice (yes vs. no) x 3 Detail (unpracticed central object vs. unpracticed central vs. practiced peripheral) mixed ANOVA demonstrated a main effect for detail, $F(2,57)=3.86, p=.02$. Follow-up paired samples $t$-tests demonstrated no significant difference between the number of commission errors for unpracticed central object details and practiced peripheral details, $t(60)<1, p=.97$. However, participants did make more commission errors for the practiced peripheral details than for the unpracticed central details, $t(60)=4.45, p<.001$, and for the unpracticed central object details than for unpracticed central details, $t(60)=5.11, p<.001$. There was no significant main effect of retrieval practice, $F(2,57)<1$, and no significant Detail $x$ Retrieval Practice interaction, $F(2,57)=2.40, p=.10$.

The data from experiment 2, in contrast to experiment 1 , show a retrieval practice effect in that repeated retrieval of peripheral details enhanced memory for these details relative to the control condition. However, repeated retrieval of (peripheral) details again did not significantly impair correct recall of unpracticed (central object) details. In other words, there was no evidence to support 
retrieval-induced forgetting, replicating the findings of experiment 1 . In contrast to the data from experiment 1, retrieval practice in experiment 2 did not result in more commission errors in the unpracticed detail categories.

A number of methodological issues merit some discussion here. First, one might question whether the retrieval practice phase in experiment 2, indeed, induced effortful retrieval for all of the retrieval practice episodes. The reason for this concern is that in the first two retrieval practice episodes participants answered closed retrieval practice questions whereas in the third episode retrieval practice questions were open (cf. Shaw et al., 1995). Thus, the first two episodes in the retrieval practice phase may have tapped recognition rather than retrieval processes. Such recognition may have required less suppression of to-beignored unpracticed central (object) details, subsequently leading to unimpaired recall of unpracticed details.

Secondly, although the results of experiment 2 did show a significant retrieval practice effect, retrieval practice success rates of the practiced peripheral details were again relatively low (34\%). This, however, should not necessarily prevent retrieval-induced forgetting from occurring. A recent study (Storm, Bjork, Bjork, \& Nestojko, 2006) showed retrieval-induced forgetting with impossible category members. Successful retrieval practice was made impossible because the two-letter stems in the retrieval practice phase did not correspond to the initial letters of any of the exemplars that were associated with the category in the study phase. Even with close to zero recall at practice, Storm et al. (2006) still obtained a retrieval-induced forgetting effect. One possibility for the present relatively low success rate is that preferential encoding of central details may have precluded successful encoding of the peripheral details in the study phase, thus leading to low retrieval practice success rates for the peripheral details. Related to this, it is possible that preferential encoding of central details was sufficiently strong that it inoculated them from putative retrieval-induced forgetting effects.

To summarize, although the present results demonstrated a retrieval practice effect, there was again no evidence for retrieval-induced forgetting, replicating the null results of experiment 1 . In contrast to experiment 1 , repeated retrieval did not result in more commission errors in unpracticed details. Retrieval- 
induced forgetting may not have emerged in experiment 2 because the relevant boundary conditions of competition and integration were not sufficiently taken into account. Furthermore, it may be that the valenced nature of the material was a barrier to obtain retrieval-induced forgetting effects. These issues will be investigated in experiment 3 .

\section{Experiment 3}

As noted, the first issue addressed in experiment 3 was the boundary condition of integration, meaning that the more the details are integrated with each other, the less likely it is for retrieval-induced forgetting to occur (e.g., Anderson \& McCulloch, 1999; Bäuml \& Kuhbandner, 2003). As discussed earlier, integration of the to-be-practiced and/or to-be-ignored exemplars might play a role either at encoding or during retrieval practice. Considering integration at encoding, experiments 1 and 2 employed an incidental study phase to attempt to minimize possible integration effects at this phase. The rationale was that being unprepared for a subsequent recall test would diminish the probability of effortful elaborative encoding of the details of the slides. However, it may be that details of a negative emotional scene elicit more spontaneous integration attempts than, say, details of neutral material, thereby making them less sensitive to retrieval practice effects. To examine whether retrieval-induced forgetting failed to occur because of the emotionally negative content of the slides, we therefore added a baseline condition in experiment 3 consisting of neutral slides that were matched with the negative ones.

As for integration during retrieval practice, the interview format of the previous experiments may have stimulated participants to integrate details. Consequently, while an interview procedure seems ecologically more valid (e.g., it is similar to police interrogations), this might not have provided the optimal method to induce retrieval-induced forgetting. We therefore decided to use a written retrieval practice procedure in experiment 3 in line with the previous literature (e.g., Anderson et al., 1994). In doing so, we expected to reduce integration and also to increase the retrieval practice success rate, i.e. the number of correctly recalled details in the retrieval practice phase.

The second issue addressed in experiment 3 concerned competition between the different detail categories within the slides. We argued earlier that peripheral 
details could be constructed as weak competitors and central details as strong competitors as a direct function of the strength of their relationship with the theme (gist) of a given slide. However, peripheral details need not necessarily to be weak competitors. Bäuml (1998) demonstrated that whether or not memory for unpracticed exemplars suffers as a function of repeated retrieval of related items, depends on the items' associative strength to the retrieval cue, with larger impairment when the category-exemplar association is strong and smaller or no impairment when the category-exemplar association is moderate or weak. The question arises therefore as to whether the central-peripheral ratings of the pilot study preceding experiment 1 are sufficient to conclude that our peripheral details indeed fulfilled these criteria for weak exemplars posited by Bäuml (1998).

One way to investigate this would be to obtain relatedness ratings of the different detail categories both with the theme of the slide, and with each other. To this end, pilot studies were conducted to explore the strength of interassociations between the detail categories and between the categories and the theme of the slide. Related to this, participants in the retrieval practice groups were asked to retrieval practice only half of the details of the central object category, leaving the remaining half unpracticed. In this way, we were able to investigate the effect of retrieval practice not only between the different detail categories (i.e., central object details vs. central details vs. peripheral details), but also within a detail category (i.e., practiced central object details vs. unpracticed central object details). It is possible that no retrieval-induced forgetting effects emerged in the previous two experiments because of differences in the associative strengths of the central object detail, central detail and peripheral detail categories, with the retrieval cues, and this modification circumvents this problem.

In sum, in experiment 3 participants were assigned to one of four groups as there were two between-group factors. These were retrieval practice - yes or no - and valence - negative or neutral slides. Based on the previous literature we hypothesized that, due to retrieval practice, the recall of central object details in the practice groups would be higher than that in the control groups. Furthermore, we predicted that retrieval practice would impair the correct recall of unpracticed central object details in the practice groups as compared to the 
control groups. Regarding valence, we hypothesized that these effects would be more pronounced in the neutral than in the negative slides, due to putative spontaneous integration effects for the valenced material.

\section{Method}

\section{Participants}

In experiment 3, 119 first- and second-year psychology students (28 men) from Maastricht University participated. Mean age was 20.61 years $(S D=3.10$; Range: 18-40). Participants were randomly assigned to one of four testing conditions. There were 30 participants in the retrieval practice neutral condition, 28 participants in the control neutral condition, 31 participants in the retrieval practice negative condition, and 30 participants in the control negative condition. The groups did not differ in mean age, $F(3,115)=2.18, p=.10$, nor in sex ratio, $\chi^{2}(1,3)=2.34, p=.51$. Participants received either course credit or $€ 7.50$ for their participation.

\section{Stimulus material}

In a pilot study $(N=15)$, we presented the five negative slides, along with ten neutral slides that were comparable with the negative slides in terms of complexity and number of details. The neutral slides were taken from different sources such as the internet and/or were composed by the experimenters. Participants were asked to rate all slides on valence and arousal on visual analogue scales (VAS; $0=$ very negative/calm $-100=$ very positive/aroused). They also indicated how many detail categories were present on the slide and rated on a VAS from $1-100$ how strongly they judged the detail categories to be related to each other. They were also asked to identify the theme of the slide and to label the detail categories that they had delineated. Other participants ( $N$ $=20$ ) rated on a VAS from $1-100$ how strongly they found the detail categories to be related to the theme of the slide, as defined by the former pilot participants.

Three pairs of negative and neutral slides were optimally matched on all of the criteria. The negative slides were the IAPS slides 3530 (Threat), 9250 (Social worker), and 9433 (Wounded man). One neutral slide was composed by the experimenters and the other two were taken from the internet. The neutral 
slides were rated as significantly more positive than the negative slides, means being $M=57.30(S D=10.30)$ and $M=13.20(S D=10.00)$, respectively, $t(14)=$ $10.98, p<.001$. The neutral slides elicited also less arousal than the negative slides, means being $M=24.67(S D=20.76)$ and $M=61.38(S D=14.74)$, respectively, $t(14)=6.13, p<.001$. Pilot participants defined three different detail categories that corresponded to central object details, other central details, and peripheral details. The total number of details for the negative slides was 30 for the central object details, 23 for the central details, and 22 for the peripheral details. This was similar for the neutral slides: 33 for the central object details, 24 for the central details, and 22 for the peripheral details. For both the negative and the neutral slides, the central object detail and central detail categories were strongly inter-associated, $76.77(S D=15.37)$ and 69.06 $(S D=18.33)$, respectively, $t(14)=1.68, p=.12$. The associative strength between central object detail and peripheral detail categories was 34.33 ( $S D=$ $14.97)$ for the negative slides and $37.34(S D=18.14)$ for the neutral slides, $t$ $(14)<1, p>.40$. The strengths of association between the central and peripheral detail categories were $40.17(S D=21.14)$ and $43.09(S D=22.77)$ for the negative and neutral slides, respectively, $t(14)<1, p>.30$.

The associative strength of the detail categories with the theme of the slide demonstrated a comparable pattern for both negative and neutral slides. For the negative slides, the central object detail category was more strongly related to the slides' theme than were the central detail category, $t(19)=5.39, p<.001$, and the peripheral detail category, $t(19)=24.27, p<.001$, means being 93.83 $(S D=5.57), 77.10(S D=13.41)$ and $26.18(S D=10.31)$, respectively. The central detail category was also significantly more associated to the theme of the slide than the peripheral detail category, $t(19)=13.50, p<.001$. This pattern of associative strength seems to correspond with Bäuml's (1998) categorization of strongly, moderately (strong), and weakly associated exemplars. For the neutral slides, the central object detail category was more strongly related to the slide theme than the central detail category, $t(19)=6.65$, $p<.001$, and the peripheral detail category, $t(19)=5.59, p<.001$, means being $74.92(S D=14.14), 54.77(S D=18.15)$ and $46.10(S D=16.37)$, respectively. However, the central detail category was not significantly more associated to the theme of the slide than the peripheral detail category, $t(19)=$ 
$1.41, p=.18$, although ratings were in the anticipated direction. These associations are less strong than those of the negative slides. The patterns of associative strengths in the negative and neutral slides appear to be in accordance with the patterns of recall usually observed in negative and neutral slides in the previous literature (e.g., Christianson, 1992).

The negative and neutral slides were given the same slide titles in order to minimize between-group differences concerning the stimulus material. Therefore, compared to experiments 1 and 2 , the negative slides had somewhat different titles. Thus, the slide titles for both the negative and neutral slides were: Together, Outside, and Metro. For each slide, the central object category, central detail category, and peripheral detail category had specific names ${ }^{9}$.

\section{Retrieval practice booklets}

For this experiment, standardized retrieval practice booklets were constructed, in which participants had to write down their answers to the retrieval practice questions. Each page contained the slide title, the name of the central object category, and a question that asked for a particular detail from the central object. Two sets of retrieval practice questions (RP-set) were constructed, and each set functioned as the retrieval practice-set for half of the participants (between-subjects counterbalancing). These RP-sets were matched on the number of details and on their association with the central object category as a whole. For the negative slides, there were 14 details in RP-set 1 and 16 details in RP-set 2. For the neutral slides, there were 16 details in RP-set 1 and 17 details in RP-set 2. Retrieval practice questions were tested in a pilot study ( $N=$ 10) to make sure that the questions were unambiguous and elicited the correct

\footnotetext{
${ }^{9}$ For all negative and neutral slides, we referred to the peripheral detail category as 'other/background details'. For the negative slide 'Metro', the central object detail category was referred to as 'attacker' and the other central detail category was referred to as 'victim'. For the neutral slide 'Metro', the central object detail category was referred to as 'man' and the other central detail category was referred to as 'woman'. In the negative slide 'Together', the central object detail category was referred to as 'two men' and the other central detail category was referred to as 'injured person'. In the neutral slide 'Together', the central object detail category was referred to as 'two men' and the other central detail category was referred to as 'man behind'. In the negative slide 'Outside', the central object detail category was referred to as 'dead man' and the other central detail category was referred to as 'bystanders'. In the neutral slide 'Outside', the central object detail category was referred to as 'man' and the other central detail category was referred to as 'bystanders'.
} 
details. Participants viewed the slides and were then asked to answer the retrieval practice questions. The results of the pilot study demonstrated that the questions elicited the answers that were expected by the experimenters. Based on the suggestions of pilot participants about the phrasing of the questions, some questions were slightly rephrased.

During the retrieval practice phase, each participant was instructed to read the questions carefully and answer them with the central object details that they remembered. They were also encouraged not to guess. Participants had $45 \mathrm{~s}$ to answer each question in the first retrieval practice episode. In the second and third episode, participants were given 30 s to answer each retrieval practice question.

\section{Distracter task}

This was exactly the same task as in experiment 2 . The alternative to the retrieval practice phase for the control participants was again the Raven task.

\section{Test booklets}

The test booklets were similar to those in experiment 2 with one exception. Apart from the slide title, participants were also given the specific names of the central object category, central detail category, and the peripheral detail category. The order of slides and categories was counterbalanced. Participants were instructed to write down all of the details that they could remember in the order of the test booklet. They were also told that they were not allowed to go back and forth within the booklet during their recall. Care was taken that the participants followed this instruction.

\section{Procedure}

With some minor modifications, the procedure was similar to that of experiment 2. First, participants were tested in small groups (maximum of five persons), with each person sitting in a cubicle with a computer. Control and retrieval practice participants were tested in separate sessions. The majority of the instructions were given in written form via the computer. Before each phase of the experiment began, the experimenter ensured that all participants understood the instructions. Second, the slides were presented for $5 \mathrm{~s}$ (as in experiment 1). 


\section{Scoring}

Cued recall data were scored by $\mathrm{BH}$ (cf., interrater reliability in experiment 1) who was blind as to whether or not participants had engaged in retrieval practice.

\section{Design}

The present study involved a 2 retrieval practice (yes vs. no) $\times 2$ valence (negative vs. neutral) $\times 2$ RP-set (set 1 vs. set 2) $\times 4$ detail (practiced central object vs. unpracticed central object vs. unpracticed central vs. unpracticed peripheral) mixed design.

\section{Results and discussion}

The mean proportion of correctly recalled central object details in the retrieval practice phase for the retrieval practice participants was $69 \%(S D=13.38)$. The mean number of reported unpracticed central object, central, and peripheral details in the retrieval practice phase was $.57(S D=.76)$. The mean number of commission errors during the retrieval practice phase was $2.47(S D=1.20)$.

Table 4.3 presents the relevant means and standard errors for the proportions of correctly recalled details and the numbers of commission errors for the different detail categories obtained during the test phase across the four groups. The 2 Retrieval Practice (yes vs. no) x 2 Valence (negative vs. neutral) x 2 RPset (set 1 vs. set 2) x 4 Detail (practiced central object vs. unpracticed central object vs. unpracticed central vs. unpracticed peripheral) mixed ANOVA yielded a main effect for detail, $F(3,111)=99.66, p<.001$, that was qualified by a significant Detail $x$ Retrieval Practice interaction, $F(3,111)=13.06, p<.001$. Follow-up independent samples $t$-tests demonstrated a significant retrieval practice effect in that the practiced central object details were significantly better recalled in the retrieval practice groups than in the control groups, $t(117)=$ $5.40, p<.001$, whereas no between-groups differences emerged in all other unpracticed detail categories, all $t$ 's $(117)<1$, all $p$ 's $>19$.

There was a main effect of valence, $F(1,111)=8.97, p=.003$, that was qualified by a borderline significant Detail $x$ Valence interaction, $F(3,111)=$ $2.49, p=.06$. 
Table 4.3. Mean proportion of correctly recalled details and number of commission errors in the test phase (standard errors are within parentheses) of the negative $(n=31)$ and neutral $(n=30)$ retrieval practice groups and the negative $(n=30)$ and neutral $(n=28)$ control groups.

\begin{tabular}{lcccc}
\hline & $\begin{array}{l}\text { Practiced } \\
\text { central object } \\
\text { details }\end{array}$ & $\begin{array}{l}\text { Unpracticed } \\
\text { central object } \\
\text { details }\end{array}$ & $\begin{array}{l}\text { Unpracticed } \\
\text { central details }\end{array}$ & $\begin{array}{l}\text { Unpracticed } \\
\text { peripheral } \\
\text { details }\end{array}$ \\
\hline $\begin{array}{l}\text { Correctly } \\
\text { recalled details }\end{array}$ & & & & \\
$\begin{array}{l}\text { Practice negative } \\
\text { Practice neutral }\end{array}$ & $.48(03)$ & $.36(.02)$ & $.22(.01)$ & $.15(.01)$ \\
Control negative & $.52(.03)$ & $.39(.03)$ & $.26(.02)$ & $.24(.02)$ \\
Control neutral & $.32(.03)$ & $.31(.03)$ & $.22(.02)$ & $.16(.02)$ \\
Commission & $.36(.03)$ & $.35(.03)$ & $.22(.02)$ & $.25(.02)$ \\
errors & & & & \\
Practice negative & $1.16(.21)$ & $.74(.14)$ & $1.94(.33)$ & $1.74(.22)$ \\
Practice neutral & $1.37(.29)$ & $.80(.18)$ & $1.73(.25)$ & $2.03(.27)$ \\
Control negative & $.77(.16)$ & $.87(.20)$ & $1.37(.23)$ & $1.80(.24)$ \\
Control neutral & $.54(.17)$ & $.68(.18)$ & $1.42(.25)$ & $1.57(.23)$ \\
\hline
\end{tabular}

Follow-up independent samples $t$-tests demonstrated that peripheral details from neutral slides were better recalled than those of negative slides, $t(117)=$ $5.64, p<.001$. No between-group differences emerged in the other practiced and unpracticed detail categories, all ts $(117)<1$, all $p s>.15$. Finally, there was a Detail $\mathrm{x}$ RP-set interaction, $F(3,111)=10.80, p<.001$. Follow-up independent samples $t$-tests showed better recall in set 1 for central object details, $t(117)=3.85, p<.001$, and for the unpracticed peripheral details, $t$ $(117)=1.96, p=.05$, but not in the unpracticed central object and central details, ts $(117)<1, p s>$.13. Furthermore, all other interaction effects, including those with RP-set as a factor, were non significant, all $F \mathrm{~s}(3,111)<1$.

Looking at the commission errors, the 2 Retrieval Practice (yes vs. no) $\times 2$ Valence (negative vs. neutral) x 4 Detail (practiced central object vs. unpracticed central object vs. unpracticed central vs. unpracticed peripheral) mixed ANOVA showed a main effect for detail, $F(3,115)=23.44, p<.001$. Follow-up paired samples $t$-tests demonstrated that participants made more commission errors for the unpracticed central detail and peripheral detail categories as compared to the central object details (irrespective of being practiced or unpracticed), $t(118)=7.74, p<.001$. There was also a main effect of retrieval practice, $F(1,115)=4.74, p=.03$, indicating that participants in the retrieval practice group made more commission errors than participants in the control group. However, no significant interaction effects were obtained, all $F$ s $(1,115)<1$. 
Taken together, although the data from experiment 3 did show a retrieval practice effect, we once more failed to find an undermining effect of retrieval practice on correct recall of unpracticed details. In other words, there was no evidence for retrieval-induced forgetting, thus replicating the null results of experiments 1 and 2. Such an effect was absent not only for negative but also for neutral slides. Retrieval practice again induced more commission errors, replicating experiment 1 , though the present data did not reveal this to be specific to unpracticed detail categories. Compared to experiments 1 and 2, the mean proportion of correctly recalled details in the retrieval practice phase in this experiment was considerably higher. However, even with this higher level of correct retrieval, we still found no reduction in correct recall for the unpracticed detail categories.

In sum, experiment 3 replicated the failure to find retrieval-induced forgetting effects from the previous two experiments after controlling for the boundary conditions of integration and competition. The results of experiment 3 also demonstrated that this failure to find retrieval-induced forgetting was not dependent on the negative valence of the stimulus material, as it was replicated with neutral slides. Finally, experiment 3 replicated the key finding of experiment 1 showing more commission errors following retrieval practice of central details.

\section{General discussion}

The aim of the present experiments was to investigate whether recall of unrehearsed details of emotional slides is poorer when other aspects of those same slides have been repeatedly retrieved. None of our three experiments demonstrated that repeated retrieval of a subset of details significantly undermines correct recall of unrehearsed details in this way. Thus, with our set of ecologically more valid stimulus material, we failed to obtain the standard retrieval-induced forgetting effect reported in the literature (e.g., Anderson et al., 1994; Barnier et al., 2004; MacLeod, 2002). However, experiment 1 did demonstrate that repeated retrieval of central object details elicited more commission errors for practiced and unpracticed details. Experiment 3 replicated these effects, also with neutral slides. Conversely, experiment 2 revealed that retrieval practice of peripheral details only enhanced memory for these details. Correct recall of unpracticed details was not affected. Neither was 
it the case that retrieval practice resulted in heightened levels of commission errors. Taken together, the present data suggest that for neutral and emotional scene material, repeated retrieval of central object details provokes commission errors for practiced and unpracticed details. At the same time, there is no support for the hypothesis that correct recall of unpracticed details would be impaired (e.g., Anderson et al., 1994; Levy \& Anderson, 2001; MacLeod, 2002).

To reassure ourselves about the validity of our inability to show that with emotionally negative slides repeated retrieval did not impair correct recall of unpracticed details, we took two relevant boundary conditions into account. First, retrieval-induced forgetting effects are unlikely to be found when practiced and unpracticed exemplars are integrated at encoding (see Anderson \& McCulloch, 1999). Consequently, to minimize possible integration effects at encoding, an incidental study phase was administered in all three experiments. In addition, to address the concern that negative slides may elicit higher levels of integration by virtue of their valence, experiment 3 included a neutral control condition. Furthermore, we reduced intertrial stimulus time from 6 to $2 \mathrm{~s}$ in experiments 2 and 3 to prevent possible integration between to-be-practiced and to-be-unpracticed details. Finally, we changed the interview-form of the retrieval practice procedure in experiments 1 and 2 to a written retrieval practice procedure that was in line with the previous literature. It is interesting to note that there is some evidence that a high degree of integration between practiced and unpracticed information induces considerable recall improvement, even of the unpracticed information (Quinn, Hugenberg, \& Bodenhausen, 2004). However, our data did not indicate enhanced memory for details in the unpracticed categories, thus providing further support for our view that integration did not play an important role.

The second boundary condition addressed in the present experiments concerned the amount of competition between practiced and unpracticed material. According to Anderson's (2003) account, when unpracticed exemplars are weakly related to the category cue, there is no need to suppress unpracticed exemplars simply because there is no competition between practiced and unpracticed information. Thus, recall of unpracticed exemplars will be unaffected. As by definition peripheral details are weakly related to the theme of the pictorial scene, it was a concern that lack of competition may have 
explained the absence of a retrieval-induced forgetting effect in experiment 1. However, it is not immediately obvious how this criticism can apply to the unpracticed central object and central detail categories in experiment 3. Ratings of associative strength with the themes of the slides were strong for the central object detail category and moderately strong for the central detail category. Thus, the absence of a retrieval-induced forgetting effect for the central (object) detail category is unlikely to be explicable in terms of weak associative strength of these categories (Bäuml, 1998).

In summary, factors such as a high degree of integration and/or a lack of competition between practiced and unpracticed information seem unlikely to account for the present failure to obtain standard retrieval-induced forgetting effects. Furthermore, a lack of retrieval-induced forgetting in our experiments cannot simply be due to a lack of retrieval practice effects as these were present in experiments 2 and 3 .

One alternative explanation for our findings concerns the manner in which the stimuli have been presented. In the typical retrieval-induced forgetting paradigm (e.g., Anderson et al., 1994), study trials are presented separately in a series of category-exemplar pairs, whereas the presentation of category cue - exemplars in the present study was necessarily simultaneous due to the nature of the material. This, together with an incidental learning instruction, may have made category cue-exemplar associations (e.g., from the negative slide Metro: Attacker-blue jumper) less prominent during both study and retrieval practice. The forming of such (episodic) cue-exemplar associations at study, as is the case with serial presentation, may be crucial for the retrieval-induced forgetting effect to emerge (see Perfect et al.'s, 2004 discussion of transfer appropriate forgetting and Racsmány \& Conway's, 2006 discussion of episodic inhibition). That is, during the retrieval practice phase a stronger pattern of associative strength may become established between some exemplars and the retrieval cue, relative to the study phase, and this may be responsible for occurrence of retrieval-induced forgetting (Perfect et al., 2004). Because such explicit (episodic) associations were not formed during study, there may not have been a chance to strengthen these associations during retrieval practice relative to the study phase in the present studies. 
Overall, the present failure to obtain typical retrieval-induced forgetting in three experiments leads us to conclude that this effect cannot easily be generalized to complex visual (emotional) scenes. The implication of this is far reaching. In our opinion, it suggests that inhibitory phenomena such as retrieval-induced forgetting may not help to explain forgetting of stimulus material that more closely resembles real life situations, as opposed to verbal stimuli such as category-exemplar pairs.

The replicated finding that repeated retrieval of central object details elicited more commission errors for practiced and unpracticed details in experiments 1 and 3 concurs with work demonstrating that extensive repeated retrieval per se increases numbers of commission errors (e.g., Henkel, 2004; Merckelbach, Zeles, van Bergen, \& Giesbrecht, 2007; Schwartz et al., 1998), and that repeated retrieval of central details induces more commission errors in peripheral details (Heuer \& Reisberg, 1990). This pattern of results may be explained in terms of relaxation of response criteria due to repeated retrieval attempts (e.g., Schwartz et al., 1998). It may also be explained in terms of attentional narrowing, which is a proposed side-effect of repeated retrieval of superiorly encoded central details and which may provoke commission errors for poorly encoded peripheral details (e.g., Candel et al., 2003; Heuer \& Reisberg, 1990). With this in mind, it is understandable that in experiment 2 retrieval practice of peripheral details did not result in more commission errors. After all, the unpracticed central details were better encoded and therefore less sensitive to possible undermining effects of repeated retrieval. Our results concerning commission errors have straightforward implications for the field of eyewitness psychology. They complement previous research indicating that repetitive interviews about negative emotional situations, such as a robbery or a car accident, can increase the number of commission errors and promotes the possible formation of false memories (e.g., Bornstein, Liebel, \& Scarberry, 1998; Roediger, Jacoby, \& McDermott, 1996).

Taken together, the present experiments relied on visual emotional material to examine whether retrieval practice of particular details undermined correct recall of other details. This was intended as a first step in extending prior research in this area (e.g., MacLeod, 2002; Wessel \& Hauer, 2006) to real-life distressing scenes and memories. Specifically, because different memory phenomena are 
said to be involved in different aspects of a single traumatic event (i.e., intrusive memory and dissociative amnesia), we employed details of complex emotional scenes rather than isolated stimuli (e.g., words or entire slides; see Anderson et al., 1994; Shaw et al., 1995). However, under these more ecologically valid circumstances, the typical retrieval-induced forgetting effect did not emerge across three separate experiments. As yet, there is therefore no evidence to suggest that retrieval-induced forgetting plays a role in remembering more complex visual emotional material, nor indeed in traumatic memories for that matter. Our data, however, do show that retrieval practice promotes commission errors, specifically when the central details have been repeatedly retrieved. 


\title{
Effect of suppressing negative memories on intrusions and autobiographical memory specificity in a non-clinical sample
}

\author{
B.J.A. Hauer, I. Wessel, E. Geraerts
}

Submitted for publication

This study investigates whether avoidance of negative autobiographical memories results in intrusions as well as overgeneral memory. Healthy participants suppressed memories of either a negative or a neutral autobiographical experience. Individuals who suppressed negative memories tended to demonstrate smaller increases in negative mood than those who did not suppress their negative target memory. However, compared to nonsuppression controls, neither suppression nor valence of the target memory predicted decreases in memory specificity. Interestingly, target memory-related intrusions during autobiographical memory retrieval predicted larger reductions in specific memory recall. The results are discussed in terms of affect regulation and other accounts explaining overgeneral memory. 


\section{Introduction}

Previous research has demonstrated that some individuals find it relatively difficult to recall specific autobiographical memories in response to cue words. This phenomenon is called overgeneral memory (Williams \& Broadbent, 1986; see for a review Williams et al., 2007). A continuing theme in the literature is the connection of traumatic life experiences with this phenomenon. For example, several studies have shown a positive relationship between traumatic experiences and overgeneral autobiographical memory in clinical samples (e.g., Dalgleish et al., 2003; de Decker, Hermans, Raes, \& Eelen, 2003; McNally, Litz, Prassas, Shin, \& Weathers, 1994) and non-clinical samples (e.g., Henderson, Hargreaves, Gregory, \& Williams, 2002). Furthermore, trauma-related symptoms such as intrusions and avoidance are significantly associated with overgeneral memory (e.g., Hermans et al., 2004; Kuyken \& Brewin, 1995; Wessel, Merckelbach, \& Dekkers, 2002). Other studies showed that particularly avoidance symptoms are associated with reduced memory specificity (e.g., Hermans, Defranc, Raes, Williams, \& Eelen, 2005; Hauer, Wessel, \& Merckelbach, 2006).

The relationship of reduced memory specificity with trauma and trauma-related symptoms has led researchers to hypothesize that overgeneral memory may be a way to regulate negative affect. Williams, Stiles, and Shapiro (1999) first proposed the affect regulation hypothesis in which they argue that traumatized individuals display overgeneral memory to protect them from the emotional distress related to trauma-memories. They theorized that at first this overgeneral retrieval strategy is restricted to the traumatic memory, but then generalizes to the whole autobiographical memory base. This hypothesis is now embedded in a broader framework that recognizes different factors that contribute to overgeneral memory (Williams, 2006; Williams et al., 2007). In the present paper, we focus on the suggested affect regulating function.

Several findings (Raes, Hermans, de Decker, Williams, \& Eelen, 2003; Raes, Hermans, Williams, \& Eelen, 2006) support the notion that an overgeneral memory retrieval style may protect individuals from experiencing negative affect that accompanies stressful events. These studies compared students who were low in specific memory retrieval with students who were highly specific in their 
autobiographical memory recall. Participants performed a negative stressful task and afterwards indicated their levels of distress. The results showed that individuals low in specific memory retrieval were less distressed after the stressful task than participants who retrieved specific memories beforehand. These findings are consistent with the view that the recall of fewer specific memories may be a way to avoid negative affect - at least in the short run. It is conceivable that a similar process would happen after experiencing a trauma.

The affect regulation hypothesis, however, is relatively silent on the precise mechanism underlying findings that overgeneral memory and trauma-related intrusions are correlated (e.g., Kuyken \& Brewin, 1995; Stokes, Dritschel, \& Bekerian, 2004; Wessel et al., 2002). Intrusive memories and thoughts about traumatic experiences are common in the aftermath of such events (e.g., Ehlers $\&$ Clark, 2000). Subsequent avoidance, i.e. by means of thought suppression, of those intrusions follows often as a secondary reaction (e.g., Ehlers \& Clark, 2000; Wenzlaff \& Wegner, 2000). Such avoidance may have two consequences in these traumatized individuals. Firstly, attempts to suppress traumatic memories may paradoxically cause more intrusions (i.e., rebound effect, Wegner, Schneider, Carter, \& White, 1987; see also Davies \& Clark, 1998; Ehlers \& Clark, 2000; Shipherd \& Beck, 2005). Secondly, attempts to avoid traumatic memories in order to reduce the negative affect that accompanies these intrusions may result in overgeneral recall of other, non-trauma related autobiographical memories. Indeed, a recent study (Schönfeld, Ehlers, Böllinghaus, \& Rief, 2007) showed that when traumatized individuals actively suppressed trauma-related thoughts during an Autobiographical Memory Test (AMT), they were more overgeneral than participants who did not actively suppress their trauma-related thoughts. Taken together, avoidance may be responsible for the repeated finding that intrusions and overgeneral memory are associated (Williams, 2006).

The present study examined whether avoidance produces more intrusions on the one hand and reduced memory specificity on the other hand. To induce avoidance of a negative autobiographical memory in non-clinical individuals, a thought suppression manipulation was employed (cf., Barnier, Levin, \& Maher, 2004; Geraerts, Merckelbach, Jelicic, \& Smeets, 2006). Participants were asked to either suppress or not suppress thoughts of their most negative 
autobiographical experience (cf., Barnier et al., 2004; Geraerts et al., 2006). In addition, to see whether the effects would be specific to avoidance of negative memories, we also included participants who either suppressed or not suppressed thoughts related to a neutral autobiographical experience.

Several hypotheses were tested. To begin with, we expected that suppression would induce a rebound effect in that the suppression groups would report more intrusions than the no-suppression control groups. In addition, we anticipated that suppression would lead to larger decreases in specific autobiographical memory retrieval than no suppression. Given the affect regulation account (Williams et al., 1999; 2007), all effects should be more pronounced following suppression of negative thoughts rather than neutral thoughts. We further hypothesized that suppression of negative memories would result in relatively low levels of negative mood (e.g., Williams et al., 2007). Finally, based on the relationship between intrusions and overgeneral memory (e.g., Kuyken \& Brewin, 1995; Wessel et al., 2002) we anticipated that more target memoryrelated intrusions during autobiographical memory retrieval would be associated with larger reductions in specific memories.

\section{Method}

\section{Participants}

Eighty-seven second-year undergraduate psychology students (27 men) from Maastricht University participated in this study. They were randomly assigned to one of four groups (see below for details): a suppression negative group ( $n=$ $21)$, a control negative group $(n=22)$, a suppression neutral group $(n=23)$ and a control neutral group $(n=21)$. The groups did not differ in sex ratio, $\chi^{2}(N=$ $87 ; d f=3)=7.42, p=.28$. Participants received course credit for their participation. The experiment was approved by the standing Ethical Committee of the Faculty of Psychology of Maastricht University.

\section{Materials}

\section{Autobiographical Memory Test (AMT)}

A Dutch translation of two versions of the AMT was used (Brittlebank, Scott, Williams, Ferrier, 1999; Wessel, Meeren, Peeters, Arntz, \& Merckelbach, 2001; 
Wessel et al., 2002; Williams \& Broadbent, 1986). Each AMT contained 10 words; 5 positive and 5 negative ${ }^{10}$. Participants were instructed to recall to each cue a different specific memory about a personally experienced event that happened at a particular time and place, and that lasted less than one day. The cues were orally and visually presented with positive and negative words alternating. Participants first practiced with neutral practice words (e.g., shop, car) until they understood the task correctly. Furthermore, they were required to respond with a specific memory within 30s (e.g., van Vreeswijk \& de Wilde, 2004). If participants gave a non-specific answer before 30s, they were prompted one time with 'Could you be more specific?'. Once the time limit was reached, the experimenter presented the next cue. The experimenter transcribed the exact response.

An independent rater assessed the first responses. Each response was coded as either a specific memory (i.e., referring to an event at a particular time and place, lasting less than a day), a non specific memory (i.e., categoric memory, an event that was about repeated occasions; extended memory, an event lasted longer than a day; no memory, a statement not being a memory), or as an omission (i.e., no response). The total number of specific memories on both AMTs was the dependent variable in this study (see Williams et al., 2007). To assess inter rater reliability, a second rater scored all responses of all participants to both AMTs (i.e., 1720 memories). Inter rater reliability for coding a memory as specific or non specific (including omissions) was good with a kappa of .73.

\section{Beck Depression Inventory-Second edition}

The BDI-II (BDI-II; Beck, Steer, \& Brown, 1996; van der Does, 2002) consists of 21 items all containing four statements that reflect depressive symptoms in

\footnotetext{
${ }^{10}$ The AMT cue words in English (Dutch) were as follows: version 1 - sorry (spijt), happy (gelukkig), angry (boos), safe (veilig), clumsy (onhandig), interested (geïnteresseerd), hurt (gekwetst), successful (succesvol), lonely (eenzaam), and surprised (verrast); version 2 - rejected (afgewezen), hopeful (hoopvol), helpless (hulpeloos), dedicated (toegewijd), guilt (schuld), calm (kalm), horrible (afschuwelijk), unconcerned (zorgeloos), sorrow (verdriet), and satisfied (tevreden).
} 
increasing severity (scored $0-3$; range $0-63$ ). Internal reliability of the BDI-II in the present sample was good, Cronbach's $a=.78$.

Impact of Event Scale (IES)

The IES (Brom \& Kleber, 1985; Horowitz, Wilner, \& Alvarez, 1979) was administered to provide a measure of frequency of intrusions and avoidance of memories related to the participant's most negative stressful experience. Internal reliability of the IES in the present sample was excellent for the IES total scale, Cronbach's $a=.91$, as well as for the avoidance, $a=.89$, and intrusion, $a$ $=.82$, subscales.

\section{Mood}

Two $100 \mathrm{~mm}$ Visual Analogue Scales (VAS) were used to measure positive and negative mood using 'gloominess' and 'happiness' with the left extreme being 'not at all gloomy/happy' and the right extreme being 'extremely gloomy/happy'.

\section{Characteristics of the target memory}

Participants rated valence, vividness, impact, and current distress after they imagined event on $100 \mathrm{~mm}$ VASs ranging from 'not at all negative/vivid/having impact/distressed' to 'extremely negative/vivid/ having impact /distressed'.

\section{Intrusion characteristics during suppression}

These were $100 \mathrm{~mm}$ VASs ranging from 'not at all ...' to 'extremely ...' asking the participants how often had event-related thoughts during the suppression or control phase (frequency), how difficult they found it not to think about the event (effort), and how successful they found themselves in suppressing event-related thoughts (success).

\section{Intrusions during $A M T$}

This was a $100 \mathrm{~mm}$ VASs ranging from 'not at all ...' to 'extremely ...' on which all participants rated how many event-related they had during the AMT (frequency).

\section{Procedure}

Participants were tested individually. They provided written, informed consent, filled in the BDI-II and the mood VASs. Subsequently, the baseline AMT was 
administered. Order of the AMTs was counterbalanced. Thereafter, all participants filled in the IES with respect to their most negative life experience.

Imagination phase

Half of the participants thought about their most negative event from the IES (negative groups). The other half thought about a neutral autobiographical experience (neutral groups). There was a list with neutral events that participants could use to remember such an event (i.e., doing groceries, walking on the street). All participants thought about their experience for $2 \mathrm{~min}$ as vividly as possible by writing it down. To warrant confidence and privacy, participants were provided with an envelop in which they could put their written thoughts, close it, and take it home. Directly after imagination, all participants filled in the VASs regarding characteristics of their target memory.

Suppression vs. control phase

This phase lasted 5min. Half of the participants (suppression groups) suppressed thoughts about their target memory (i.e., either their most negative or a neutral experience). These participants were instructed to close their eyes and not to think about the target memory. They could think about anything else, but not about their target memory. The other half (control groups) was instructed that they could think about anything, including their target memory. All participants monitored their thoughts and pressed a button every time they had thoughts related to their target memory. Immediately after the suppression or control phase, all participants filled in the VASs about the intrusion characteristics.

Next, all participants rated their current mood on VASs and thereafter the postsuppression AMT was administered. During the AMT, all participants again monitored their thoughts and pressed a button every time they were having a thought about their target memory. Subsequently, they filled in the VAS about intrusion frequency. Finally, participants were thanked for their participation and given course credit.

\section{Design and data analysis}

The design of the present study was a 2 (Suppression: yes vs. no) x 2 (Valence of the to-be-suppressed memory: negative vs. neutral) between subjects design 
using measures of intrusions and autobiographical memory specificity as the dependent variables ${ }^{11}$. Throughout the results section, we refer to the four individual groups as: Suppress-Neg (suppression negative group), Suppress$\mathrm{Neu}$ (suppression neutral group), Control-Neg (control negative group) and Control-Neu (control neutral group).

\section{Results}

Table 5.1 presents the self-report questionnaire data for the four different groups. In the whole sample, mean BDI-II score was 5.59 ( $S D=4.44$; Range 023). Thirteen participants scored in the range of mild to moderate depression (10-18) and one in the range of moderate to severe depression (19-29). Mean IES scores were 16.90 ( $S D=15.03$; Range 0-58), 8.76 ( $S D=8.20$; Range 0-35) and $8.14(S D=8.13$; Range 0-30) for the intrusion and avoidance subscales, respectively. Fifty-one participants scored in the non-clinical range (IES $<19$; Horowitz et al., 1979) and 11 participants in the likely PTSD range (IES > 35; Neal et al., 1994; Wohlfarth, van den Brink, Winkel, \& ter Smitten, 2003). Groups did not significantly differ on the BDI-II, the IES total, intrusion and avoidance subscales, all $F s<1$. Forty-six participants noted death or illness of loved one or self as their most negative experience. Furthermore, interpersonal problems $(n=20)$, threat to self or loved one $(n=11)$, and other experiences such as accidents were reported $(n=9)$. On average, the event happened 51 months ago $(M d n=34 ; S D=46.36)$.

\section{Characteristics of target memories}

Table 5.1 also presents participants' ratings about the characteristics of the target memory. As expected, the negative groups rated their target memory as more negative than the neutral groups, $t(84)=12.31, p<.001, d=2.64$. The negative groups also rated their target memory as having more impact than the

\footnotetext{
${ }^{11}$ One person in the suppression negative group indicated that he had not actually suppressed the event. This person was removed from the sample, leaving 20 participants in the suppression negative group. Additionally, one person in the control neutral group indicated having forgotten to button press during the control phase. This person did fill in the VASs about the intrusion characteristics. This results in a lower $d f$ number when the analyses are reported with the number of button presses during the suppression period.
} 
Table 5.1. Means (SDs) of self-reported symptoms of depression (BDI-II), intrusions and avoidance (IES) as well as characteristics of the target memory (all VASs) in the four different groups.

\begin{tabular}{lcccc}
\hline & $\begin{array}{l}\text { Suppress-Neg } \\
(\boldsymbol{n}=\mathbf{2 0})\end{array}$ & $\begin{array}{l}\text { Suppress-Neu } \\
(\boldsymbol{n}=\mathbf{2 3})\end{array}$ & $\begin{array}{l}\text { Control-Neg } \\
(\boldsymbol{n}=\mathbf{2 2})\end{array}$ & $\begin{array}{l}\text { Control-Neu } \\
(\boldsymbol{n}=\mathbf{2 1})\end{array}$ \\
\hline $\begin{array}{l}\text { Self-reported } \\
\text { symptoms }\end{array}$ & & & & \\
BDI-II & $6.15(5.18)$ & $6.39(5.61)$ & $5.64(3.36)$ & $5.59(4.44)$ \\
IES total & $16.37(16.36)$ & $18.36(18.52)$ & $14.50(13.66)$ & $18.32(11.00)$ \\
IES intrusions & $8.77(9.81)$ & $8.49(8.57)$ & $8.32(8.00)$ & $9.52(6.72)$ \\
IES avoidance & $7.60(7.26)$ & $9.87(11.31)$ & $6.18(6.58)$ & $8.80(6.02)$ \\
Characteristics & & & & \\
of target memory & & & & \\
Valence (VAS) & $61.25(22.66)$ & $13.04(17.06)$ & $61.27(23.37)$ & $7.32(12.17)$ \\
Impact (VAS) & $55.55(22.35)$ & $9.78(13.30)$ & $60.27(18.82)$ & $14.14(20.68)$ \\
Distress (VAS) & $36.30(27.18)$ & $3.87(6.67)$ & $37.18(26.47)$ & $4.29(6.24)$ \\
Vividness (VAS) & $66.55(19.97)$ & $71.57(14.53)$ & $72.82(18.90)$ & $72.10(15.19)$ \\
\hline
\end{tabular}

Note. Suppress-Neg = suppression negative group; Suppress-Neu = suppression neutral group; Control-Neg = control negative group; Control-Neu = control neutral group; VAS = Visual Analogue Scale; BDI-II = Beck Depression Inventory-II; IES = Impact of Event Scale.

neutral groups, $t(84)=11.36, p<.001, d=2.45$. Additionally, they were more distressed about it than the neutral groups, $t(84)=7.84, p<.001, d=1.67$. As expected, there was no significant difference between the negative and neutral groups in vividness of the target memory, $t(84)<1$.

Intrusions during the suppression phase

Table 5.2 presents the number of intrusions (button presses) during the suppression phases as well as ratings of intrusion characteristics. A 2 (Suppression: yes vs. no) $\times 2$ (Valence: negative vs. neutral) between subjects ANOVA with the number of button presses as the dependent measure showed a significant main effect of suppression, $F(1,81)=4.54, p<.05, d=.44$. Thus, both suppression groups reported fewer intrusions than both control groups. There was no significant main effect of valence, nor a significant Suppression $\mathrm{x}$ Valence interaction, $F S<1$. Similar results were obtained with frequency and success as dependent measures, but no significant results emerged regarding effort not to think about the experience.

\section{Effect of suppression on mood}

Table 5.2 presents the mood data obtained at baseline and after suppression. We computed mood change scores to control for mood at baseline by subtracting mood at baseline from mood after suppression. A 2 (Suppression: yes vs. no) $\times 2$ (Valence: negative vs. neutral) between subjects ANOVA with 
Table 5.2. Mean (SDs) numbers of self-reported mood before and after suppression as well as measures of intrusions during suppression and the post-suppression AMT in the four different groups.

\begin{tabular}{|c|c|c|c|c|}
\hline & $\begin{array}{l}\text { Suppress- } \\
\text { Negative } \\
(n=20)\end{array}$ & $\begin{array}{l}\text { Suppress- } \\
\text { Neutral } \\
(n=23)\end{array}$ & $\begin{array}{l}\text { Control- } \\
\text { Negative } \\
(n=22)\end{array}$ & $\begin{array}{l}\text { Control- } \\
\text { Neutral } \\
(n=21)\end{array}$ \\
\hline \multicolumn{5}{|l|}{$\begin{array}{l}\text { Intrusions during } \\
\text { suppression }\end{array}$} \\
\hline Button presses & $3.55(4.05)$ & $5.70(6.13)$ & $6.45(5.12)$ & $7.95(6.60)$ \\
\hline Frequency (VAS) & $23.90(23.88)$ & $28.91(27.92)$ & $35.50(22.13)$ & $44.67(29.07)$ \\
\hline Effort (VAS) & $39.20(23.30)$ & 45.35 (30.22) & $50.18(25.23)$ & $50.57(33.28)$ \\
\hline Success (VAS) & $52.80(26.90)$ & $54.09(27.36)$ & $43.45(25.73)$ & $37.95(19.90)$ \\
\hline \multicolumn{5}{|l|}{$\begin{array}{l}\text { Intrusions during } \\
\text { post-suppression } \\
\text { AMT }\end{array}$} \\
\hline Button presses & $1.75(2.31)$ & $1.83(3.02)$ & $2.64(3.46)$ & $3.76(4.43)$ \\
\hline Frequency (VAS) & $18.35(20.70)$ & 9.65 (14.66) & $19.18(25.69)$ & $23.95(27.05)$ \\
\hline \multicolumn{5}{|l|}{$\begin{array}{l}\text { Mood pre- } \\
\text { suppression }\end{array}$} \\
\hline Positive (VAS) & 53.05 (22.63) & $65.65(15.21)$ & $64.77(9.90)$ & $66.14(14.56)$ \\
\hline Negative (VAS) & $19.15(18.10)$ & 12.09 (12.23) & $10.77(13.14)$ & $19.52(18.78)$ \\
\hline \multicolumn{5}{|l|}{$\begin{array}{l}\text { Mood post- } \\
\text { suppression }\end{array}$} \\
\hline Positive (VAS) & $51.70(19.83)$ & $63.65(17.64)$ & $59.00(14.80)$ & $65.67(15.43)$ \\
\hline Negative (VAS) & $23.40(18.21)$ & $9.96(11.01)$ & $25.14(20.19)$ & $15.24(17.79)$ \\
\hline
\end{tabular}

Note. AMT = Autobiographical Memory Test; Suppress-Neg = suppression negative group; Suppress-Neu = suppression neutral group; Control-Neg = control negative group; Control-Neu = control neutral group; VAS = Visual Analogue Scale.

negative mood change as the dependent measure showed a main effect of valence, $F(1,82)=14.07, p<.01, d=.81$, but not of suppression, $F(1,82)=$ $1.42, p=.22$. There was a borderline significant Suppression $\times$ Valence interaction, $F(1,82)=3.38, p=.07, \eta=.04$, suggesting that participants who suppressed thoughts about their negative target memory showed smaller negative mood increases than those participants who did not suppress thoughts about their negative target memory. Similar analyses with positive mood change as the dependent measure showed no significant main or interaction effects, Fs $<1$.

\section{Effect of suppression on intrusions during the AMT}

Table 5.2 also presents the number of intrusions while carrying out the AMT. A 2 (Suppression: yes vs. no) $\times 2$ (Valence: negative vs. neutral) between subjects ANOVA with the number of intrusions (button presses) as the dependent measure revealed a borderline significant main effect of 
suppression, $F(1,82)=3.70, p=.06, d=.42$, suggesting that both suppression groups still tended to report fewer event-related intrusions during autobiographical memory retrieval than both control groups. There was no significant main effect of valence, nor a significant Suppression $x$ Valence interaction, $F_{S}<1$. No significant results emerged on self-reported frequency of intrusions.

\section{Effect of suppression on autobiographical memory specificity}

Table 5.3 presents the autobiographical memory specificity data at baseline and after suppression. To examine effects of suppression on autobiographical memory specificity, a difference score was computed by subtracting AMT baseline scores from AMT scores after suppression. A 2 (Suppression: yes vs. no) $x 2$ (Valence: negative vs. neutral) $\times 2$ (AMT-order: version 1-2 vs. version 2-1) mixed ANOVA with memory specificity difference as the dependent variable revealed a significant effect of AMT-order, $F(7,78)=7.13, p<.001$, showing a larger decreases in memory specificity when version 2 was presented after the manipulation ${ }^{12}$. Furthermore, there were no significant main effects of suppression or valence, neither a significant Suppression $x$ Valence interaction or any interactions with AMT-order, all $F_{S}<1$. Similar results were obtained with specific memories to positive or negative cues as separate dependent measures, all $F_{S}<1^{13}$.

\section{Association of intrusions during AMT with autobiographical memory specificity}

Finally, we examined whether intrusions during the AMT would be related to larger reductions in memory specificity. A regression analysis was computed including suppression, valence and order of AMT as control measures and

\footnotetext{
${ }^{12}$ Even though order of AMT was counterbalanced, it appears that version 2 was more difficult in this sample. Nevertheless, we do not further interpret this interaction.

${ }^{13}$ The content of the memories reported after suppression was examined within the Suppress-Neg and Control-Neg groups. The mean number of reported memories related to the individuals' most stressful experience was $.68(S D=.89)$ in the Suppress-Neg group and $.50(S D=.83)$ in the Control-Neg group. An independent samples $t$-test demonstrated that there was no significant difference between the groups, $t<1$.
} 
Table 5.3. Mean (SDs) numbers of specific and non-specific memories and omissions during autobiographical memory retrieval on the baseline and post-suppression AMTs in the four different groups.

\begin{tabular}{lllll}
\hline & $\begin{array}{l}\text { Suppress- } \\
\text { Negative } \\
(\boldsymbol{n}=\mathbf{2 0})\end{array}$ & $\begin{array}{l}\text { Suppress- } \\
\text { Neutral } \\
(\boldsymbol{n}=\mathbf{2 3})\end{array}$ & $\begin{array}{l}\text { Control- } \\
\text { Negative } \\
(\boldsymbol{n}=\mathbf{2 2})\end{array}$ & $\begin{array}{l}\text { Control- } \\
\text { Neutral } \\
(\boldsymbol{n}=\mathbf{2 1})\end{array}$ \\
\hline $\begin{array}{l}\text { Autobiographical memory } \\
\text { specificity (AMT) at baseline }\end{array}$ & & & & \\
$\begin{array}{l}\text { Specific } \\
\text { Non-specific }\end{array}$ & $6.85(1.09)$ & $6.52(2.23)$ & $6.55(1.65)$ & $7.24(1.34)$ \\
$\begin{array}{l}\text { Omissions } \\
\text { Autobiographical memory }\end{array}$ & $1.80(1.01)$ & $1.70(1.36)$ & $2.14(1.36)$ & $1.24(1.18)$ \\
specificity (AMT) after & $1.35(1.35)$ & $1.78(1.68)$ & $1.32(1.09)$ & $1.52(1.40)$ \\
suppression & & & & \\
$\begin{array}{l}\text { Specific } \\
\text { Non-specific }\end{array}$ & & & \\
$\quad$ Omissions & $6.05(2.14)$ & $6.74(1.86)$ & $5.95(2.06)$ & $6.33(1.94)$ \\
\hline
\end{tabular}

Note. AMT $=$ Autobiographical memory Test; Suppress-Neg = suppression negative group; Suppress-Neu = suppression neutral group; Control-Neg = control negative group; Control-Neu = control neutral group.

intrusions (button presses) during the AMT as the independent variable of interest. Memory specificity difference was the dependent measure. The overall model was significant, $F(4,81)=3.64, p<.001, R=.39, R^{2}=.15$. Not surprisingly, suppression, $\beta=.04, t<1$, and valence, $\beta=.12, t=1.13, p=.26$, were non significant, whereas order of AMT, $\beta=.27, t=2.64, p=.01$, predicted reductions in memory specificity. Interestingly, intrusions during the AMT, $\beta=$ .23, $t=2.16, p=.03$, significantly were also related to decreases in memory specificity. Thus, more intrusions about the target memory during autobiographical memory retrieval were associated with larger decreases in specific memories. A similar pattern of results emerged for memory specificity to positive cues, $F(4,81)=8.71, p<.001, R=.55, R^{2}=.30$. Again, while controlling for suppression, valence and order of AMT, intrusions during the AMT were a highly significant predictor of reductions in memory specificity, $\beta=$ .32, $t=3.32, p=.001$. However, the model was no longer significant with memory specificity to negative cues, $F(4,81)<1$.

\section{Discussion}

The present aim was to examine the role of avoidance in the relationship between intrusions and overgeneral memory. Individuals were asked to suppress thoughts related to a personal experience in order to explore the effects on subsequent intrusions and overgeneral memory. The suppression 
manipulation was successful in that those individuals who suppressed their event reported fewer intrusions during suppression than those who did not suppress it. However, unlike prior findings (e.g., Davies \& Clark, 1998; Geraerts et al., 2006; Harvey \& Bryant, 1998; but see Kelly \& Kahn, 1994) there was no rebound effect. In fact, the opposite was true. Rather than showing an increase in intrusions, the suppression groups tended to show a further decrease during autobiographical memory retrieval. Additionally, in contrast to Schönfeld et al. (2007), the suppression manipulation did not result in a larger reduction of autobiographical memory specificity. However, after controlling for suppression and valence of the to-be-suppressed memory, more intrusions during postsuppression autobiographical memory retrieval were associated with larger reductions in specific memories, specifically to positive cue words. This finding extends prior research (e.g., Kuyken \& Brewin, 1995; Wessel et al., 2002) in that intrusions related to an active memory - either negative or neutral - are associated with larger decreases in autobiographical memory specificity. Taken together, the present data seem not to concur with the idea that avoidance underlies the relationship between intrusions and overgeneral memory.

The idea that avoidance plays a pivotal role in the association between intrusions and overgeneral memory was inspired by the affect regulation account (Williams et al., 1999; 2007) which states that overgeneral memory retrieval is a way to avoid negative affect related to negative experiences. Interestingly, there was a trend in the data showing that particularly those individuals who did not suppress thoughts related to their negative event experienced larger negative mood increases than participants in the other groups. Put differently, individuals who did suppress negative target memoryrelated thoughts showed no mood increase. Instead, they experienced a negative mood level that was comparable to that of the neutral groups. This result agrees with earlier studies supporting beneficial short-term effects of avoidance as an affect regulating mechanism (e.g., Raes et al., 2003; 2006; Williams et al., 2007).

Yet, the memory results seem not consistent with the idea that affect regulation leads to overgeneral memory. Overall, both personally negative and neutral intrusions, whether they were suppressed or not, were significantly related to larger reductions in specific memories, particularly to positive cue words. 
According to affect regulation, only negative intrusions should be related to reduced memory specificity. Therefore, the present result may concur better with other findings explaining simultaneous occurrence of intrusions and overgeneral memory in terms of reduced executive functioning (e.g., Dalgleish et al., 2007). In this experiment, monitoring of intrusions during the AMT can be regarded as a dual task, which may have primarily affected the relatively demanding task to retrieve specific memories.

The current results might seem at odds with those of Schönfeld et al. (2007), who found that suppression of trauma-related thoughts resulted in more overgeneral memory. Though, our study differed from Schönfeld et al. in that in our study thought suppression preceded autobiographical memory retrieval, whereas they induced suppression during the AMT. In addition, Schönfeld et al. argued that - instead of the actual act of suppression per se - their results may be explained by a greater task demand on the AMT that was created by the thought suppression instruction. This concurs with our explanation for the present association between intrusions about the target memory during the AMT and larger reductions in specific memory recall.

This study is not without some methodological limitations. Firstly, the number of specific memories was somewhat lower than in other non-clinical student samples (e.g., Hauer et al., 2006; Hermans et al., 2005). A methodological difference is that here the AMT was administered orally and participants had 30 s to respond with a specific memory, whereas the other studies used a written format and participants had 60s to respond. van Vreeswijk and de Wilde (2004), however, showed that time to respond does not seem to moderate memory specificity. Secondly, the level of traumatic experiences was relatively low, which may have mitigated possible outcome effects in that fewer intrusions were elicited after suppression. Even so, participants did sincerely recall their most negative experience, the type of event was serious (i.e., death/illness, assault, threat of loved ones or the self, or interpersonal problems like divorce of parents) and there was sufficient variance in reported symptomatology. The use of a community sample with somewhat older individuals who have experienced more negative events might be a good suggestion for future research. 
All in all, the present findings extend prior research in two ways. To begin with, the result that, relative to no suppression, thought suppression of memories from negative experiences did not result in a larger negative mood increase supports the view that avoidance of negative experiences may be beneficial in the short run (e.g., Raes et al., 2003; 2006). Secondly, irrespective of suppression and valence of the target memory, those individuals who reported more intrusions about their target memory during autobiographical memory retrieval demonstrated larger reductions in specific memory recall. This result extends prior findings that trauma-related intrusion and overgeneral memory recall are associated (e.g., Kuyken \& Brewin, 1995; Wessel et al., 2002). Finally, the present results emphasize the importance of a framework including differential variables explaining the occurrence of overgeneral memory (Williams, 2006; Williams et al., 2007). 



\title{
Autobiographical memory specificity after manipulating retrieval cues in adults reporting childhood sexual abuse
}

\author{
B.J.A. Hauer, I. Wessel, E. Geraerts, H. Merckelbach, T. Dalgleish, T. \\ Journal of Abnormal Psychology (in press)
}

Research has shown that traumatized samples have relative difficulty in generating specific autobiographical memories on a cue word task, compared to non-exposed controls. Simultaneously, trauma is associated with a preponderance of highly specific intrusive trauma memories in day-to-day life. A possible explanation for this pattern is that day-to-day intrusions and memories generated to cue words rely on different retrieval processes, with the former dependent on close associations between retrieval cues and specific memory representations (direct retrieval), and the latter on iterative retrieval cycles through a hierarchical memory system (generative retrieval). The present study sought to operationalize this distinction in the laboratory using two versions of the cue word task, designed to promote generative and direct retrieval respectively, in participants with or without a history of child sexual abuse (CSA). The data confirmed our hypothesis that CSA participants would be less specific than non-abused controls to the generative retrieval cues, but that this difference would disappear with the direct retrieval cues. This interaction was stronger in the CSA participants with relatively greater posttraumatic stress, remained significant when participants with past or current major depressive disorder were excluded, and also when only those participants with corroborated CSA were included. The implications of these data for understanding relatively distinct forms of memory retrieval following trauma are discussed. 


\section{Introduction}

Numerous studies suggest that individuals with emotional disorders have problems accessing specific aspects of their personal past. For example, Williams and Broadbent (1986) asked depressed suicide attempters and healthy controls to generate specific autobiographical memories to a series of emotion-related cue words (i.e., the Autobiographical Memory Test [AMT]). They found that the parasuicide group was far more likely to produce 'overgeneral' responses (e.g., to the word party, an overgeneral response might be 'I have never enjoyed parties'), than to reply with memories of specific experiences (e.g., 'I enjoyed Jane's party last Saturday'). Such difficulty in producing specific memories on the AMT has since been demonstrated in various clinical groups (see Williams et al., 2007, for a review), with the majority of studies focusing on depressed states (e.g., Brittlebank, Scott, Williams, \& Ferrier, 1993; Kuyken \& Dalgleish, 1995; Wessel, Meeren, Peeters, Arntz, \& Merckelbach, 2001). Extending this research into the domain of trauma, Kuyken and Brewin (1995) showed that depressed women reporting childhood abuse showed reduced specificity relative to depressed women reporting no abuse. This relationship between a history of trauma and reduced memory specificity has since been replicated numerous times and following various kinds of traumatic events (see Moore \& Zoellner, 2007, for a review).

The affect regulation hypothesis

Theoretical conceptualizations of reduced autobiographical memory specificity have proposed various causal processes (Williams, 2006; Williams et al., 2007), grounded in mainstream cognitive models of autobiographical recall (e.g., the Self Memory System; Conway \& Pleydell-Pearce, 2000). In the context of trauma, a predominant and compelling view of the reduced specificity effect has been the Affect Regulation Hypothesis (Williams, Stiles, \& Shapiro, 1999). The proposal here is that trauma-exposed individuals experiencing trauma-related distress seek to avoid remembering the specific details of their traumatic experiences in an attempt to reduce that distress. This avoidant memory style, it is proposed, then generalizes beyond the recollection of the trauma memories themselves until it comes to characterize much broader domains of 
autobiographical remembering, resulting in reduced specificity on tests such as the AMT which do not explicitly target trauma memories.

The Affect Regulation Hypothesis thus provides a plausible account of reduced memory specificity on the AMT in distressed trauma survivors. However, it fails to account for why those survivors continue, in many instances, to report frequent intrusive and very specific memories of their trauma in their daily life (Brewin, Christodoulides, \& Hutchinson, 1996; McNally, 2003), which at their most disabling, form the core of psychiatric conditions such as Posttraumatic Stress Disorder (PTSD; American Psychiatric Association, 1994). In other words, why are the same populations that routinely show relatively reduced memory specificity on the AMT nevertheless characterized by unwanted memories of specific autobiographical trauma-related material?

One possibility is that day-to-day trauma-related intrusions on the one hand, and reduced specificity on the AMT on the other hand, are the products of different kinds of memory retrieval processes (Brewin, Dalgleish, \& Joseph, 1996; Conway \& Pleydell-Pearce, 2000; Williams et al., 2007). For example, it has been suggested that the reduced specificity effect on the AMT is a result of disruption in the effortful "generative" retrieval of memories within a hierarchically organized memory architecture such as the Self Memory System referred to above (Conway \& Pleydell-Pearce, 2000; Williams et al., 2007). Generative retrieval within this model relies upon top-down memory search processes involving the use of conceptual representations to form the basis for the search. Once search criteria are specified, general information (referring to lifetime periods, e.g. 'when I lived in Cambridge', or to particular classes of events, e.g. 'my vacations in France') at the top of the hierarchy is rapidly activated. Activation then spreads through the autobiographical knowledge base from such general event representations to representations of specific autobiographical experiences, at the bottom of the hierarchy. Within this theoretical context, reduced memory specificity results from premature truncation of the generative search for specific memories, leading to retrieval of general autobiographical summaries from higher up the hierarchy. In the case of trauma, then, this truncated search would represent the generic avoidant memory style that is at the heart of the Affect Regulation Hypothesis. 
In contrast, it has been proposed that intrusive recollection of specific trauma memories in daily life results from a process of "direct" autobiographical memory retrieval (Brewin et al., 1996; Conway \& Pleydell-Pearce, 2000; Conway, Singer, \& Tagini, 2004; Williams et al., 2007; see also, Burgess \& Shallice, 1996). Direct retrieval refers to situations where an internal or environmental cue produces immediate activation of a specific memory, thus bypassing any generative search. Importantly, this bypassing of the generative search process ensures that direct retrieval of memories, including trauma memories, is unaffected by any premature truncation of generative retrieval occurring in the service of affect regulation.

In summary, the theoretical proposal is that the Affect Regulation Hypothesis is operationalized via truncated generative autobiographical memory retrieval in distressed trauma survivors (Williams et al., 2007), leading to difficulty accessing any specific material by this means. In contrast, retrieval of any specific memories via direct means, including retrieval of trauma memories, remains unaffected by such truncation of generative search processes.

The principal aim of the present study was to examine this putative distinction in the laboratory, by contrasting performance on the standard version of the AMT (proposed to broadly elicit generatively retrieved responses) with performance on an AMT constructed so as to broadly elicit directly retrieved responses. In this way, we can examine whether the usual reduced memory specificity effect on the standard AMT in distressed trauma survivors, relative to controls, is significantly reduced or attenuated on an AMT designed to promote relatively direct retrieval processes. It is important to stress that these versions of the AMT do not need to focus on retrieval of trauma memories because the theoretical arguments outlined above propose that all generative retrieval will be relatively compromised and that all direct retrieval will be relatively spared in distressed, trauma survivors.

To construct an AMT designed to promote direct retrieval, we considered existing theoretical proposals about memories that are putatively directly retrieved (Brewin et al., 1996; Conway \& Pleydell-Pearce, 2000). One potential reason for direct retrieval is the presence of an extensive repertoire of direct associations between cues in the internal and external environment and specific 
representations in memory. Such associations could have become established as a function of the frequency with which memories have been accessed in response to certain cues, and thus represent the relative automatization of memory access (Moors \& de Houwer, 2006). Another non-mutually-exclusive possibility is that direct retrieval is more likely for memories stored as imagebased mental representations (e.g., Brewin et al., 1996; Conway \& PleydellPearce, 2000). Such memories would be directly accessed by cues or reminders that either contain or are strongly associated with image-based information that overlaps with, or is highly-related to, the content of the representations.

These potential processes of automaticity and of facilitated specific memory retrieval using highly imageable cues were investigated by Williams, Healy and Ellis (1999). These authors showed that manipulating the imageability of the cue words on the AMT led to marked differences in the proportions of specific memories recalled, with concrete, highly imageable cues leading to significantly greater specificity, consistent with the recruitment of image-based processes during recollection. Furthermore, retrieval of specific memories to these highly imageable cues was relatively immune to the effects of mental load, thus suggesting a degree of automatization (Williams, Chan, Barnhofer, Eade \& Healy, 2006, Study 3).

On the basis of these theoretical ideas and accompanying data, we opted in the present study for an AMT methodology with cues varying in imageability as a vehicle with which to try to operationalize the putative contrast between relatively direct and generative autobiographical recall in trauma-exposed individuals outlined above. In line with this, the first main hypothesis for the present study was that a trauma-exposed sample would show reduced memory specificity relative to non-trauma-exposed controls on the standard version of the AMT which uses generally low imageable cues (Williams \& Broadbent, 1986), but that this group difference would be significantly attenuated or eliminated on an AMT consisting of highly imageable, concrete cue words designed to promote direct retrieval.

A further key finding in the AMT and trauma literature is that, within traumaexposed samples, those individuals with higher levels of post-traumatic stress 
or with PTSD show more marked reductions in specificity on the standard AMT compared with those with less distress or no PTSD (see Moore \& Zoellner, 2007). In fact, there is a well-replicated negative correlation between levels of intrusion of specific trauma memories in day-to-day life and levels of memory specificity on the AMT (e.g., Kuyken \& Brewin, 1995; Wessel, Merckelbach, \& Dekkers, 2002).

The prototypical account of these relationships (e.g. Williams et al., 2007) is that higher distress (including more frequent intrusions) following trauma results in a greater emphasis on affect regulation via truncated generative retrieval, and thus reduced specificity on the standard AMT. However, ironically, the intrusions that are a key source of the distress in the first place are largely unaffected by these regulation attempts by virtue of the fact that they are habitually directly retrieved and, as we have sought to argue, direct retrieval is relatively unaffected by truncated generative search.

Our second hypothesis sought to draw upon these theoretical claims. We predicted that within our trauma-exposed group, those survivors with clinically significant levels of posttraumatic stress (according to established cut-offs on the Impact of Event Scale; Horowitz, Wilner \& Alvarez, 1979), would show a more marked reduction in memory specificity on the standard AMT compared to those survivors with low levels of symptoms. In contrast, on the version of the AMT designed to promote direct retrieval, we predicted that this group difference would be attenuated or eliminated as there is no reason to suppose that levels of traumatic distress should significantly impact on specificity of directly retrieved general autobiographical material.

To investigate the hypotheses outlined above, we chose to recruit a sample of adults reporting a history of Childhood Sexual Abuse (CSA), along with current intrusive symptomatology. This seemed an appropriate population because adult participants abused as children were the initial research focus in the trauma and AMT literature (Kuyken \& Brewin, 1995) and the reduced specificity finding on standard versions of the AMT has proven to be robust in this population (Moore \& Zoellner, 2007). Furthermore, in theoretical terms it has been argued that childhood represents a critical period when individuals are 
highly susceptible to developing this particular memory style as a form of functional avoidance (Williams et al., 1999).

The recruitment of a CSA sample allowed us to address a supplementary hypothesis emerging out of the current AMT literature (Williams et al, 2007). Williams et al. noted that a significant limitation of the AMT findings with abused individuals to date (e.g., Kuyken \& Brewin, 1995) was that in no instance was corroboration of the abuse reported. Consequently, our supplementary hypothesis (Hypothesis 3) was that those among our CSA participants for whom their history of abuse was corroborated would show reduced specificity relative to our non-CSA controls on the standard version of the AMT. Indeed, it seems reasonable to presume that the interaction effect specified in Hypothesis 1 should also be present across these two groups.

\section{Reduced memory specificity and depression history}

To our knowledge, no studies describing reduced autobiographical memory specificity effects in traumatized samples have controlled for both past and current clinical depression. There are some studies that have controlled for current major depressive disorder (MDD; e.g., McNally et al., 2006; Schönfeld \& Ehlers, 2006), but in these studies past MDD was not taken into account. As MDD is a common outcome following trauma (e.g., Shalev et al., 1998), this is a non-trivial problem with the existing data in the literature as it means that in all of the studies to date reporting reduced specificity associated with trauma, history of MDD and trauma are confounded. As MDD history has been found to be associated with reduced memory specificity, even in the absence of a current MDD diagnosis and even when self-reported depressive symptoms have been controlled for (e.g., Mackinger, Pachinger, Leibetseder, \& Fartacek, 2000), this leaves open the possibility that any apparently trauma-related effects on the AMT are somehow a function of group differences in MDD history that have not been adequately assessed (see the Methodological Limitations section in Williams et al.'s, 2007, review of these findings).

In the present study, we therefore sought to compare a sub-sample of the individuals with CSA who had never been clinically depressed, with a subsample of similarly never-clinically-depressed, non-CSA controls to resolve this confound. Our fourth hypothesis was consequently that the never-depressed 
CSA group would show reduced memory specificity relative to the neverdepressed non-CSA controls on the standard AMT, after also covarying current levels of self-reported depressed mood. Indeed, we presumed that the interaction effect specified in Hypothesis 1 should also be present across these never-depressed sub-groups. If this fourth hypothesis is supported by the data, this would indicate for the first time that trauma is significantly related to reduced specificity independent of both current and past MDD.

\section{Method}

\section{Participants}

CSA participants $(n=70 ; 57$ women; mean age $=41.37$ years; $S D=11.59)$ were recruited through advertisements in provincial newspapers inviting participants to come to the laboratory if they had a history of CSA, of which they reported a continuous memory (i.e., participants reported that they had always remembered their CSA experiences). CSA was defined as sexual contact ranging from sexually related fondling to penetrative acts before the age of 12 (McNally, Clancy, Barrett, \& Parker, 2004; 2005). In a standard semi-structured interview (McNally et al., 2004; 2005), participants reporting CSA were asked a number of questions about the nature of their abuse (e.g., when it happened, duration, and who the perpetrator was). All of the CSA participants reported current intrusive symptomatology.

Control participants ( $n=63 ; 41$ women; mean age $=39.06$ years; $S D=12.57$ ), group-matched with the CSA participants for age and education, were recruited through further advertisements describing research into personality and memory. Participants were only included in the control group if they denied having a history of CSA in the semi-structured interview (McNally et al., 2004; 2005).

The Dutch version of the Structured Clinical Interview for the DSM-IV (SCID; Groenestijn, Akkerhuis, Kupka, Schneider, \& Nolen, 1999) was used to assess MDD in both the CSA and the control group. In the CSA group, 10 participants $(14.3 \%)$ had a current major depressive episode. Thirty-one participants (44.3\%) reported a history of MDD. Only one participant who had past MDD had a current major depressive episode. SCID-PTSD was also completed for those 
CSA participants with significant Criterion B symptoms of nightmares, flashbacks and intrusive thoughts or images. There were 15 CSA participants (21.4\%) who had current PTSD with these Criterion B symptoms. Six of these participants were also diagnosed with current MDD. Furthermore, three participants with current PTSD had former MDD. Abused participants were asked for corroboration of their abuse, either by other family members or by providing documentary evidence (e.g., letters, medical records). Thirty participants $(42.9 \%)$ gave the name of a person that could corroborate the abuse (e.g., a sibling that was also abused), and confirmation was forthcoming in all cases. Two further participants $(2.8 \%)$ provided a court document indicating that the perpetrator had been convicted for the abuse. In the control group, nobody was diagnosed with current MDD. There were eight participants $(12.7 \%)$ with previous MDD.

All participants received $€ 50$ and reimbursement of traveling expenses after their participation. This study was approved by the standing ethical committee of Maastricht University.

\section{Materials}

\section{Autobiographical Memory Test (AMT)}

Two versions of the AMT (Williams \& Broadbent, 1986) were used, each containing 10 words; 5 positive and 5 negative (available from the corresponding author). A Dutch translation (Wessel et al., 2001) of the original version of the AMT (the standard AMT) from Williams and Broadbent (1986) was compared to a version (the concrete AMT) comprising highly imageable, concrete cue words (e.g., funeral, cuddle). Words for the concrete AMT were selected on the basis of a pilot study $(N=13)$, in which 25 positive and negative words were rated on 9-point scales assessing imageability, concreteness, and how readily participants felt the words would bring to mind specific memories. The scales ranged from 1 (not at all visually imageable; extremely abstract; does not easily elicit a specific memory) to 9 (extremely visually imageable; extremely concrete; extremely easily elicits specific memory). Similar ratings were made for the standard AMT words. On the basis of these ratings, 10 words for the concrete AMT were selected such that they were significantly more imageable $(M=7.85 ; S D=.51$ vs. $M=5.06 ; S D=.51$, respectively), $t(12)$ 
$=7.45, p<.001$, more concrete $(M=7.77 ; S D=.55$ vs. $M=4.60 ; S D=1.20$, respectively), $t(12)=7.60, p<.001$, and elicited specific personal memories more readily than the standard words $(M=7.55 ; S D=.88$ vs. $M=6.14 ; S D=$ .76 , respectively), $t(12)=6.71, p<.001^{14}$.

On each AMT, participants were instructed to recall to each cue a specific memory about a personally experienced event that happened at a particular time and place, and that lasted less than one day. Participants were asked to provide a different memory for each cue. The cue words were orally and visually presented with positive and negative words alternating. To familiarize participants with the procedure, a maximum of 10 neutral practice words (e.g., shop, car, trip, conversation) were given until participants recalled at least three specific memories in direct succession. Participants were required to respond with a specific memory within 60s. If participants gave a non-specific answer before the 60 s, they were prompted one time with 'Could you be more specific?' Once the time limit was reached, the experimenter presented the next cue word. The experimenter transcribed the exact response.

The first responses given to the cue words on the AMT were coded by an independent rater. Each response was coded as either a specific memory (i.e., referring to an event at a particular time and place, lasting less than a day), a general categoric memory (i.e., a group of similar events that had occurred on repeated occasions), a general extended memory (i.e., a single event lasting longer than one day), no memory (i.e., a coherent response that was not a memory; for example, a simple semantic association to the cue word), or an omission (i.e., no response). The total number of different specific memories retrieved to the 10 cue words for each version of the AMT was the primary dependent variable in the present study. However, for the first hypothesis we carried out a representative analysis with numbers of general categoric memories. A second rater scored all of the responses to the standard and

\footnotetext{
${ }^{14}$ Although the present study is not concerned with valence effects, it is useful to note that based on similar rating scales, the concrete words did not significantly differ from the standard words on positive valence $(M=7.33 ; S D=.58$ vs. $M=7.38 ; S D=.75$, respectively), $t(12)<1, p>.70$ and negative valence $(M=3.85 ; S D=.55$ vs. $M=3.51 ; S D=.88$, respectively), $t(12)=1.91, p>.08$, and on emotional intensity $(M=6.31 ; S D=.1 .20$ vs. $M=6.11 ; S D=.70$, respectively $), t(12)<1, p$ $>.50$.
} 
concrete AMTs of a random sample of 20 participants (i.e., 400 memories) to assess inter-rater reliability. Inter rater reliability for coding a memory as specific or non specific was good with a Kappa of .70 .

Beck Depression Inventory-Second edition (BDI-II)

The BDI-II consists of 21 items all containing four statements that reflect depressive symptoms in increasing severity (scored 0-3; range 0-63; Beck, Steer, \& Brown, 1996; van der Does, 2002). Internal reliability of the BDI-II in the present sample was excellent, Cronbach's $a=.94$.

Impact of Event Scale (IES)

The IES (Brom \& Kleber, 1985; Horowitz et al., 1979) was administered to the CSA group to provide a measure of frequency of post-traumatic stress symptoms. The IES is a widely used instrument to measure frequency of intrusions and avoidance of memories related to the participant's most negative stressful experience. This could be the CSA or another experience. Internal reliability of the IES in the present sample was excellent for the IES total scale, Cronbach's $a=.93$, and also for the intrusions and avoidance subscales, $a=$ .91 and $\mathrm{a}=.88$, respectively.

\section{Procedure}

Participants first provided written, informed consent. They then completed the two versions of the AMT in a counterbalanced order, with a $15 \mathrm{~min}$ distractor task (i.e., an unrelated questionnaire) in between. After the two AMTs, the semistructured interview and the SCID were administered. Thereafter, participants filled in the BDI-II, the IES, and some other questionnaires not reported here. Finally, CSA participants were asked for any corroboration of their abuse.

\section{Results}

\section{Demographic data}

Table 6.1 presents the demographic and the self-report questionnaire data for the CSA and control groups. The groups did not differ in terms of age or education, though they did differ on gender ratio. The CSA group scored 
Table 6.1. Demographic variables, and means (SDs) of self-reported symptoms of depression (BDIII), intrusions and avoidance (IES) for the CSA and control groups.

\begin{tabular}{lllll}
\hline & $\begin{array}{l}\text { CSA group } \\
(\boldsymbol{n}=\mathbf{7 0})\end{array}$ & $\begin{array}{l}\text { Control group } \\
(\boldsymbol{n}=\mathbf{6 3})\end{array}$ & Test statistic & Significance \\
\hline $\begin{array}{l}\text { Demographic variables } \\
\text { Mean age }\end{array}$ & $41.37(11.59)$ & $39.06(12.57)$ & $t(131)=1.10$ & $\begin{array}{l}p=.27 \\
\text { \% Women }\end{array}$ \\
$\begin{array}{l}\text { Mean education a } \\
\text { Self-reported symptoms }\end{array}$ & $5.40(1.73)$ & 65.1 & $\chi^{2}(1)=4.57$ & $p=.03$ \\
BDI-II & $12.51(12.37)$ & $4.46(5.07)$ & $t(130)=4.81^{\mathrm{b}}$ & $p<.001$ \\
IES total & $26.86(20.51)$ & $13.95(14.02)$ & $t(125)=4.04$ & $p<.001$ \\
IES intrusions & $13.75(10.66)$ & $7.88(7.79)$ & $t(125)=3.49$ & $p=.001$ \\
IES avoidance & $13.10(11.34)$ & $6.02(7.23)$ & $t(125)=4.08$ & $p<.001$ \\
\hline
\end{tabular}

Note. ${ }^{a}$ (ranging from $1=$ elementary school to $7=$ academic degree); ${ }^{b}$ One participant in the CSA group did not fill in the BDI-II and the IES. In the control group, five participants did not fill in the IES. For this reason, the degrees of freedom are lower in the analyses when these measures are used. $\mathrm{CSA}=$ childhood sexual abuse; BDI-II = Beck Depression Inventory-II; IES = Impact of Event Scale.

significantly higher than the controls on the BDI-II. Fifty-four participants (77\%) in the CSA group filled in the IES with respect to their CSA experience. The remaining 16 cited a range of traumas including death or illness, accident, and threat to beloved others or to the self. The IES scores of the CSA group were comparable to those reported in previous studies of trauma-exposed samples (de Decker, Hermans, Raes, \& Eelen, 2003; Stokes, Dritschel, \& Bekerian, 2004). Control participants filled in the IES with respect to their most negative stressful event. Thirty-two participants $(51 \%)$ reported death or illness to the self or beloved others as their most negative event. The other control participants cited different traumas including accident or threat to beloved others or to the self. Ten control participants completed the IES but did not report a reference event. For the whole sample, and for the CSA and control groups considered separately, the IES (including the subscales) and BDI were significantly intercorrelated, $r s>.26, p s<.05$.

\section{AMT data}

Tables 6.2 and 6.3 present the AMT data across the different CSA and control groups. Again, the various sub-samples drawn from the CSA and control groups did not significantly differ regarding age and educational level. As for gender, the only sub-samples that significantly differed in gender ratio were the CSA sub-sample who filled in the IES with respect to their abuse and the control group, as well as the never depressed CSA and never depressed control groups. Yet, initial analyses including gender rendered no significant main 
effects or interactions involving this factor, all $F_{S}<1$. The same was true for initial analyses conducted with order of AMT presentation (standard-concrete vs. concrete-standard) as a factor. Therefore, all further analyses are reported without order and gender as factors.

\section{Psychometric equivalence of the two AMT tasks}

A concern when examining differential degrees of group difference across tasks is that any significant interactions may simply reflect differences in the discriminating power of the tasks at hand (e.g., Chapman \& Chapman, 1973; 1978). We therefore calculated the true variance of the two versions of the AMT as an index of discriminating power (Chapman \& Chapman, 1973). True variance can be estimated by calculating the product of the internal reliability and the observed variance for each measure from a sample of healthy control participants. Using this method, the discriminating powers of the standard AMT and the concrete AMT, based on the scores of the controls $(n=63)$ in the present study, were 3.32 and 2.65, respectively. Interestingly, the internal reliabilities for both versions of the AMT were virtually indistinguishable, Cronbach's a's $=.64$. For this reason we could statistically compare the two measures of true variance by simply comparing the observed variance using an F-Ratio Test. On this basis, the two measures did not significantly differ from each other, $p=.37$. Thus, we were able to conclude that there was no support for the two AMTs being different from each other in terms of estimated discriminating power (e.g., Chapman \& Chapman, 1973; 1978), thus giving us confidence that any significant interaction effects represented differential group differences that were unlike to simply be psychometric epiphenomena.

\section{Hypothesis 1: CSA vs. Controls}

Table 6.2 presents the AMT data across the CSA and control groups. A 2 (group: CSA vs. control) by 2 (AMT: standard vs. concrete) ANOVA with numbers of specific memories as the dependent variable was performed to investigate our first hypothesis that the CSA group would show reduced memory specificity on the standard AMT compared to controls, but that this group difference would be significantly attenuated or eliminated on the concrete AMT. 
Table 6.2. Mean (SDs) numbers of specific, general categoric, and general extended memories, no memories, and omissions (SDs) on the standard and concrete autobiographical memory tasks (AMT) in the child sexual abuse (CSA) and control groups.

\begin{tabular}{lcccc}
\hline & \multicolumn{2}{c}{ CSA group $(\boldsymbol{n}=\mathbf{7 0})$} & \multicolumn{2}{c}{ Control group $(\boldsymbol{n}=63)$} \\
& Standard AMT & Concrete AMT & Standard AMT & Concrete AMT \\
\hline Specific & $4.84(2.77)$ & $7.03(2.01)$ & $6.24(2.28)$ & $7.41(2.04)$ \\
Specific same event & $.17(.45)$ & $.06(.29)$ & $.05(.21)$ & $.02(.13)$ \\
General categoric & $1.23(1.11)$ & $.59(.91)$ & $.40(.81)$ & $.31(.60)$ \\
General extended & $1.33(1.50)$ & $.61(.79)$ & $.71(.76)$ & $.80(.87)$ \\
No memory & $1.80(1.87)$ & $1.34(1.54)$ & $1.73(1.66)$ & $1.36(1.77)$ \\
Omission & $.63(1.05)$ & $.37(.85)$ & $.78(1.12)$ & $.11(.31)$ \\
\hline
\end{tabular}

There were significant main effects of AMT, $F(1,131)=85.20, p<.001, \eta^{2}=$ .35 , and of group, $F(1,131)=6.25, p=.01, \eta^{2}=.04$. These were qualified by a significant Group x AMT interaction, $F(1,131)=7.71, p=.006, \eta^{2}=.06$. Independent samples $t$-tests demonstrated that the groups differed significantly on the standard AMT, $t(131)=3.15, p=.002, d=.55$, but not on the concrete AMT, $t(131)=1.09, p>.20$, in support of our hypothesis. The CSA group scored significantly higher on the BDI-II than the controls and there were significant negative correlations between memory specificity and BDI-II scores across the whole sample, standard AMT, $r(132)=-.31, p<.05$; concrete AMT, $r(132)=-.26, p=.003$. Therefore, above analysis was repeated with the BDI-II as a covariate, producing an identical pattern of results as those above ${ }^{15}$.

As noted in the materials section, for this main analysis, we cross-checked the pattern of findings using numbers of categoric memories as the dependent

\footnotetext{
${ }^{15}$ Some authors have argued that ANCOVA is often misused when dealing with covariates that may be related to the group factor when group membership is not random (see Miller \& Chapman, 2001). This is potentially relevant in the present study because it is plausible that CSA participants are more depressed than control participants for reasons that are directly associated with their history of abuse. In other words, membership of the CSA group might not be independent of being somewhat depressed. This means that covarying depressive symptoms from the group variable in the present analysis would only allow us to draw conclusions about whatever is residual about membership of a CSA group, once the variance associated with depressed mood has been accounted for. However, as Miller and Chapman (2001) also argue, if one is less concerned about what the residual group variable might actually represent in ANCOVA, and is simply motivated to discover whether this residual variable still produces significant effects of interest following covariation, then ANCOVA is justified. These were the circumstances of the present analyses where we simply wanted to reassure ourselves that the significance of the effects associated with the group variable was not entirely carried by group differences in depressed mood.
} 
variable. A 2 (group: CSA vs. control) by 2 (AMT: standard vs. concrete) ANOVA generated the same pattern of results as those with specific memories as the dependent measure, with significant main effects of $\operatorname{AMT}, F(1,131)$ $=15.83, p<.001, \eta^{2}=.11$, and group, $F(1,131)=16.34, p<.001, \eta^{2}=.06$, qualified by a significant Group x AMT interaction, $F(1,131)=8.71, p=.004, \eta^{2}$ $=.11$. Independent samples $t$-tests demonstrated that the groups differed significantly on the standard AMT, $t(131)=4.57, p<.001, d=.85$, but not on the concrete AMT, $t(131)=1.69, p=.09$. Again, including BDI-II in the analyses did not change the results. For the remainder of the analyses, we report only those findings involving numbers of specific memories as the dependent variable. However, the patterns of findings were comparable to those findings involving numbers of specific memories when categoric memories were used.

As a minority of the CSA group $(n=16)$ did not identify abuse as their index trauma on the IES, the above analyses were repeated with these participants excluded. Mean specificity and categoric data for the remaining CSA participants $(n=54)$ are presented in Table 6.3. The results of these analyses with and without BDI-II as a covariate yielded the same patterns of significant results to those previously reported.

Table 6.3. Mean (SDs) numbers of specific and general categoric autobiographical memories on the standard and concrete autobiographical memory tests (AMT) in sub-samples of the child sexual abuse (CSA) and control groups.

\begin{tabular}{lcccl}
\hline & \multicolumn{2}{c}{ Standard AMT } & \multicolumn{2}{c}{ Concrete AMT } \\
& Specific & Categoric & Specific & Categoric \\
\hline Control-ND $(\boldsymbol{n}=\mathbf{5 5})$ & $6.33(2.25)$ & $.40(.81)$ & $7.40(2.10)$ & $.31(.60)$ \\
CSA IES abuse $(\boldsymbol{n = 5 4 )}$ & $4.57(2.78)$ & $1.24(1.08)$ & $7.04(1.99)$ & $.52(.86)$ \\
CSA-IES high $(\boldsymbol{n = 2 3 )}$ & $3.65(2.17)$ & $1.43(.90)$ & $6.52(2.15)$ & $.43(.66)$ \\
CSA-IES low $(\boldsymbol{n}=\mathbf{2 6})$ & $5.42(2.90)$ & $1.04(1.11)$ & $7.27(1.97)$ & $.65(1.09)$ \\
Control-IES low $(\boldsymbol{n}=\mathbf{3 8})$ & $6.63(2.48)$ & $.50(.89)$ & $7.23(2.21)$ & $.32(.70)$ \\
CSA-ND $(\boldsymbol{n}=\mathbf{2 8})$ & $4.78(2.79)$ & $1.14(1.18)$ & $7.32(1.83)$ & $.71(1.15)$ \\
CSA-C $(\boldsymbol{n}=\mathbf{3 2})$ & $4.50(2.64)$ & $1.38(1.18)$ & $7.22(1.75)$ & $.69(1.06)$ \\
\hline
\end{tabular}

Note. IES = Impact of Event Scale; Control-ND = sub-sample of never depressed control participants; CSA IES abuse = CSA participants who filled in the IES with respect to the abuse; CSA-IES high = sub-sample of CSA participants with IES scores of $>35$; CSA-IES low = subsample of CSA participants with IES scores of < 19; Control-IES low = sub-sample of control participants with IES scores of < 19; CSA-ND = sub-sample of never depressed CSA participants; $\mathrm{CSA}-\mathrm{C}=$ sub-sample of CSA participants who had corroboration for their abuse. 
Hypothesis 2: CSA with and without significant PTSD symptomatology (vs. controls)

To investigate our second hypothesis we divided our CSA group on the basis of their IES scores into those falling within Horowitz et al.'s (1979) non-clinical range (using the cut-off of $<19$ ) (CSA-IES low; $n=26$ ), and those falling within the likely PTSD range (CSA-IES high; $n=23$ ) identified in more recent studies (using the cut-off of $>35$; Neal et al., 1994; Wohlfarth, van den Brink, Winkel, \& ter Smitten, 2003). These CSA IES-high and CSA IES-low groups did not significantly differ in age, $t(47)<1$, education, $\chi^{2}(6)=3.50, p>.60$, or gender ratio, $\chi^{2}(1)=.05, p>.50$.

We compared the performance of these CSA groups with that of control participants with IES scores $<19$ (controls-IES low; $n=38$ ) on the two AMT tasks (see Table 6.3), with a 3 (group: CSA-IES high vs. CSA-IES low vs. controls-IES low) by 2 (AMT: standard vs. concrete) ANOVA with numbers of specific memories as the dependent measure. The results revealed significant effects of group, $F(2,84)=5.80, p=.004, \eta^{2}=.12$, and AMT, $F(1,84)=65.18$, $p<.001, \eta^{2}=.44$, and a significant Group x AMT interaction, $F(2,84)=9.49, p$ $<.001, \eta^{2}=.18$.

To deconstruct the interaction term we examined the relevant patterns of paired comparisons using independent samples $t$-tests. On the standard AMT, the CSA-IES high group was significantly less specific than the CSA-IES low group, $t(48)=2.57, p=.01, d=.74$, but the two groups did not differ on the concrete AMT, $t<1$, thus replicating the pattern of findings involving the whole CSA group vs. controls reported above. Unsurprisingly, the CSA-IES high group also differed from the control-IES low group on the standard AMT, $t(58)=4.97, p<$ $.001, d=1.32$, and again the groups did not differ on the concrete AMT, $t<1$, thus reproducing the same pattern. Finally, there was a borderline significant difference between the CSA-IES low and the control-IES low groups on the standard AMT, $t(62)=1.88, p=.07$, but not on the concrete AMT, $t<1$, 
reflecting a significant AMT $\mathrm{x}$ Group interaction between these two low IES groups, $F(1,62)=5.88, p<.02, \eta^{2}=.04^{16}$.

As for the whole study sample, within the CSA group memory specificity was significantly negatively correlated with self-reported depression on the BDI-II, standard AMT, $r(69)=-.25, p=.04$; concrete AMT, $r(69)=-.38, p=.001$. Consequently, the above analyses were repeated with BDI-II scores as a covariate, producing an identical pattern of results.

We also examined the correlations between IES scores and numbers of specific memories, this time within the CSA group as a whole. Spearman's correlations were used as the IES scores were not normally distributed. For the standard AMT, the number of specific memories was significantly negatively correlated with IES-avoidance, $r(67)=-.26, p<.04$, but not with IES-intrusion scores, $r$ (68) $=-.14, p=.25$, although the latter were in the anticipated direction. For the concrete AMT, IES scores did not significantly correlate with specificity: IESavoidance subscale, $r(67)=-.16, p=.19$; IES-intrusion subscale, $r(69)=-.07$, $p=.57$. These data broadly reflect the findings using the IES cut-offs reported above ${ }^{17}$.

\section{Hypothesis 3: Corroborated CSA vs. controls}

Table 6.3 also presents the AMT data for CSA participants with corroboration for their abuse (CSA-C; $n=32$ ). A 2 (group: CSA-C vs. controls) x 2 (AMT: standard vs. concrete) ANOVA with specific memories as the dependent variable revealed significant main effects of AMT, $F(1,93)=93.00, p<.001$,

\footnotetext{
${ }^{16}$ Interestingly, as can be seen from Table 6.3, this was a crossover interaction. This provides further evidence that the differential performance across the two tasks is not simply a function of their psychometric properties (Chapman \& Chapman, 1973).

${ }^{17}$ As a final examination of the relationship between IES scores, BDI scores, and specific memories we carried out regression analyses to examine the unique contributions of the scores on the two self-report measure (as predictors) to the memory scores (as dependent variables). For the standard AMT, the overall model was significant, $F(2,129)=7.77, P<.001$, accounting for $11 \%$ of the variance in memory scores, and the standardized $\beta$ s and part correlations were: IES $-\beta=-.12$, $r_{\text {part }}=-.09, t(127)=1.11$, ns; BDI $-\beta=-.24, r_{\text {part }}=-.18, t(127)=2.11, P<.05$. For the concrete AMT, the overall model was not significant $F(2,129)=2.34, n s$, and accounted for only $4 \%$ of the variance in memory scores.
} 
$\eta^{2}=.38$, and of group, $F(1,93)=5.39, p=.02, \eta^{2}=.11$. These were qualified by a significant Group x AMT interaction, $F(1,93)=11.27, p=.001, \eta^{2}=.05$. Subsequent independent samples $t$-tests demonstrated that the CSA-C group was less specific on the standard AMT than controls, $t(93)=3.33, p=.001, d=$ .70 , but not on the concrete AMT, $t(93)<1, p>.60$, supporting our hypothesis. Again, the analyses yielded the same pattern of results with BDI-II score covaried.

\section{Hypothesis 4: Never depressed CSA vs. never depressed controls}

Table 6.3 shows the AMT data for never depressed CSA participants (CSA-ND; $n=28$ ) and for never depressed controls (controls-ND; $n=55$ ). To investigate our third hypothesis, we conducted a 2 (group: CSA-ND vs. control-ND) by 2 (AMT: standard vs. concrete) ANOVA with number of specific memories as the dependent measure. There was a main effect of AMT, $F(1,81)=56.12, p<$ $.001, \eta^{2}=.35$, and a strong trend for a main effect for group, $F(1,81)=3.08, p$ $=.08, \eta^{2}=.03$. The key AMT $\times$ Group interaction was also significant, $F(1,81)$ $=9.23, p=.003, \eta^{2}=.10$. Independent samples $t$-tests again revealed that the CSA-ND individuals were less specific than the control-ND group on the standard AMT, $t(81)=2.71, p=.008, d=.61$, but not on the concrete AMT, $t$ $(81)<1, p>.80$. These results therefore support our hypothesis. As before, the relevant analyses remained significant with $\mathrm{BDI}$ - II scores included as a covariate.

\section{Discussion}

The first hypothesis of the present study was that participants reporting childhood sexual abuse (CSA) would exhibit significantly reduced memory specificity compared to non-CSA controls on the standard version of the Autobiographical Memory Task (AMT), chosen to elicit relatively generative memory retrieval, but that this difference would be significantly attenuated or absent on a concrete-word version of the AMT, designed to promote relatively direct retrieval of memories (Conway \& Pleydell-Pearce, 2000; Williams et al., 2007). The data supported this hypothesis, even after covarying levels of depressed mood on the BDI-II and even when analyses were restricted to those individuals reporting corroboration of their abuse. To our knowledge, this is the first demonstration that the reliable reduced memory specificity effect can be 
eliminated in a trauma-exposed population, relative to controls, by manipulating the content of the cue words on the AMT.

The second hypothesis was that a similar pattern would emerge when comparing those survivors in the CSA group with clinically significant levels of posttraumatic stress (on the Impact of Event Scale) with those survivors with minimal symptoms. Again the data supported our hypothesis, even with selfreported depression on the BDI-II was controlled. This pattern was also mirrored in correlational analyses using the IES in the CSA group. These data are in accordance with the findings from a study by Schönfeld and Ehlers (2006), published after we began the present research. Schönfeld and Ehlers compared groups of participants with and without PTSD on the numbers of general memories they retrieved on a standard cue word AMT and on a version in which the cues were affective pictures. They found that the PTSD participants were significantly less general in response to the pictures than to the words, with no significant difference for the no-PTSD participants. However, in contrast to the pattern in the present study, Schönfeld and Ehlers found that their PTSD group remained more overgeneral than the no-PTSD controls, even on the picture AMT (Schönfeld, personal communication) ${ }^{18}$. Possibly, picture cues match less well with individual's memories than words cues because they are too specific and therefore still elicit generative retrieval.

The findings pertaining to the above hypotheses are potentially important in two ways. First, the fact that the same trauma-exposed participants were either less specific relative to controls or were non-significantly different from controls, dependent on the cueing context (with comparable findings for high versus low symptom trauma-exposed groups), is consistent with theoretical conceptualizations of autobiographical recollection in distressed trauma survivors (Williams et al., 2007) that propose relatively distinct retrieval processes that operate under different circumstances. One possible implication of the present data is that processes that disrupt relatively generative retrieval of

\footnotetext{
${ }^{18}$ It is important to note there was also no significant Group $x$ Task interaction for numbers of specific memories in the Schönfeld and Ehlers (2006) dataset and that the significance of the interaction involving general memories did not survive controlling for verbal IQ.
} 
memories, such as affect regulation (Williams et al., 1999), have little or no effect on relatively direct recall of the personal past. If, as we assume, intrusive re-experiences of the trauma in trauma survivors are examples of relatively directly retrieved memories and that such retrieval is promoted by using the concrete word version of the AMT, this argument would suggest that processes of affect regulation targeted to avoid distressing specific autobiographical material are in fact missing the primary source of such distress in the form of these intrusive trauma memories.

Evidence in support of this possibility is provided by Golden, Dalgleish and Mackintosh (in press) who showed that individuals who had experienced a traumatic bereavement and were suffering from complicated grief (CG) did not show the usual reduced specificity effect, relatively to bereaved non-CG controls, on a cue word task (the Biographical Memory Task; BMT) designed to probe memories from the lifetime of the deceased loved one (rather than the self). In contrast, they did show the usual reduced specificity effect on the standard AMT and on a BMT with reference to the life of a loved one who was still alive. As memories of the deceased form the content of day-to-day distressing intrusions in CG sufferers, these data provide evidence supporting the view that retrieval of such memories is relatively immune to putative processes of affect regulation that operate on the standard AMT.

The present data and those of Golden et al. (in press) do not necessarily mean that affect regulation in the form of reduced memory specificity is wholly dysfunctional for traumatized individuals (in particular those with significant symptoms), simply that it is imperfect. For example, it may be that such 'avoidance' processes still mitigate the trauma-related distress experienced by survivors by sufficiently reducing access to trauma-related material via relatively generative routes, and/or by reducing the retrieval frequency of other emotive memories. This is a matter for future research. However, it is important to note that even if these putative affect regulation processes do effectively compromise relatively generative recall of the trauma, this itself may be dysfunctional in the medium- to long-term. For example, there is a growing body of research indicating that successful and repeated generative recall of traumatic events as a function of prolonged exposure techniques is broadly beneficial in terms of the amelioration of posttraumatic stress (e.g., Ehlers, Clark, Hackmann, McManus, 
\& Fennell, 2005; Pennebaker, 1995). Furthermore, reduced autobiographical specificity is related to poor problem solving strategies (e.g., Goddard, Dritschel, \& Burton, 1996; 1997).

The finding that a significant reduced specificity effect on the standard AMT can be eliminated by selecting different cue words (and attenuated by using pictures, Schönfeld \& Ehlers, 2006) also has a potentially important methodological implication. It highlights the fact that care needs to be taken in selection of cue materials for future AMT experiments to ensure that the appropriate memory retrieval context is invoked (see also Williams et al., 2006; Williams et al., 1999).

Our third hypothesis was that the difference in specificity on the standard AMT between CSA participants and controls would remain significant when we included only those from the CSA sample with corroborated abuse. The data supported this hypothesis and, to our knowledge, this represents the first demonstration of the standard AMT effect involving a corroborated abuse sample.

The final hypothesis of the present study was that the sub-sample of our CSA group with no current or past MDD would be less specific on the standard AMT compared to never-depressed, non-CSA controls, even after covarying current levels of depressed mood on the BDI-II. The data confirmed this hypothesis and indeed the pattern of findings in support of Hypothesis 1 was also replicated across these sub-samples. This gives us more confidence when interpreting the reduced memory specificity effect as it relates to trauma independent of MDD, in the service of making theoretical arguments within this literature.

It is important to note that we are not trying to argue that reduced specificity effects in traumatized populations (such as the current CSA group) are not influenced by depressed mood or other symptoms of depression. Indeed, in the present data self-reported depressive symptoms on the BDI-II were negatively correlated with memory specificity, both across the sample as a whole and within the CSA group, on both concrete and standard versions of the task. This is perhaps not surprising given the high degree of inter-correlation between traumatic stress and depressive symptoms. Rather, what we have sought to do in the current analyses is demonstrate that there remains a significant 
relationship between AMT performance and trauma once the influence of depression is controlled for, both by selecting participants with no history of MDD, and by covarying self-reported depressive symptoms on the BDI-II.

The present study is not without some limitations. The most significant is that we included no independent way of assessing that the words in our concrete AMT invoked 'direct retrieval', as far as one can actually validate such theoretical constructs (see the Introduction). It may therefore be the case that for some participants and/or for some cue words in the concrete AMT condition, the resulting retrieval processes were not 'direct' (Conway \& Pleydell-Pearce, 2000). However, to the extent that this was the case it would have gone against the grain of our principal hypothesis (leading to reductions in memory specificity in the CSA group for these words on the concrete AMT). Consequently, this issue is perhaps less of a concern for the present study.

The fact that we did not assess direct versus generative retrieval explicitly in the present study of course means that we have no data that verify that the key difference between the two AMT tasks maps closely onto this theoretical distinction. It would be highly desirable for future studies to explicitly assess the extent to which retrieval to highly imageable word cues reflected proposed theoretical components of direct retrieval such as automaticity or access via image-based representations. Such studies could use manipulations of mental load, could assess the sensory properties of the retrieved memories, or examine the time course of retrieval to different cues. Indeed, once the notions of direct versus generative retrieval can be deconstructed in terms of such other variables, it may be that the broader notions themselves prove redundant. This is a matter for further study.

A further limitation of the current study is that we did not carry out a complete assessment of trauma history in our control group, as our primary concern was to establish absence of self-reported CSA. It may be that some of the controls had experienced significant non-CSA traumas with associated effects on their AMT performance. Again, however, this would have gone against the grain of our main hypothesis of a group difference on the standard AMT, relative to the concrete AMT, and so is of less concern for the present study. 
A final limitation is the absence of reliability data for the SCID. Furthermore, the full set of PTSD questions for the SCID was only administered to those participants endorsing intrusion-related Criterion B symptoms. This therefore precluded formal comparisons between CSA participants with versus without PTSD. However, we were able to perform analogous comparisons with high versus low PTSD symptom groups using established cut-offs on the IES, and the results were in the line with the study hypotheses.

In conclusion, the present study showed that the widely-replicated finding of significantly reduced autobiographical memory specificity in trauma-exposed individuals, relative to controls, can be eliminated by the use of cue words designed to promote relatively direct autobiographical memory retrieval, with a similar pattern in high- versus low-symptom-level trauma survivors. The study also provided the first evidence that we know of that the reduced memory specificity effect associated with CSA is present independently of current and past MDD, and after controlling for current depressed mood. 



\title{
Prepartum autobiographical memory specificity predicts posttraumatic stress symptoms following complicated pregnancy
}

\author{
B.J.A. Hauer, I. Wessel, I.M. Engelhard, L.L. Peeters, T. Dalgleish.
}

Submitted for publication.

Prior research has shown that reduced autobiographical memory specificity predicts an increase in posttraumatic stress severity in traumatized individuals. Studies have also demonstrated that pre-trauma overgeneral memory predicts symptoms of depression after pregnancy-related life stress. So far, no reported studies tested the predictive value of pre-trauma overgeneral memory on posttraumatic stress. Therefore, the aim of the present study was to investigate whether prenatal overgeneral memory would predict posttraumatic stress after complicated pregnancy. The results demonstrate that women who retrieved fewer specific memories with a pregnancy-related content to positive cues during pregnancy (i.e., directly after hospitalization), reported more posttraumatic stress six weeks after giving birth. This relationship remained significant after covarying variables that were related to both baseline overgeneral memory and later posttraumatic stress. A similar pattern was found for depression symptomatology, even when somatic symptoms were excluded from the analyses. Taken together, these data suggest that the relationship of pre-trauma overgeneral memory with later depression can be generalized to posttraumatic stress symptoms. 


\section{Introduction}

There is a wealth of evidence that individuals suffering from posttraumatic stress find it relatively difficult to recall specific autobiographical memories in response to cue words (e.g., death, party) on the Autobiographical Memory Test (AMT; Williams \& Broadbent, 1986), compared with less distressed trauma survivors and with those with no history of trauma exposure (e.g., Hauer, Wessel, Geraerts, Merckelbach \& Dalgleish, in press; McNally, Lasko, Macklin, \& Pitman, 1995; Schönfeld \& Ehlers, 2006; see Moore \& Zoellner, 2007 for a review). Similar results have consistently been found for patients suffering from mood disorders (see Williams et al., 2007).

An important reason why such reduced specificity in autobiographical memory has attracted significant research attention is that it independently predicts poorer symptom outcomes in longitudinal studies in a range of clinical and subclinical groups (see Williams et al., 2007). For example, individuals with unipolar depression or with seasonal affective disorder who show relatively reduced memory specificity when depressed, go on to show more depressive symptoms when followed up several months later (e.g., Brittlebank, Scott, Williams, \& Ferrier, 1993; Dalgleish, Spinks, Yiend, \& Kuyken, 2001; Mackinger, Pachinger, Leibetseder, \& Fartacek, 2000; though see Brewin, Reynolds, \& Tata, 1999).

To our knowledge, only one study has shown that reduced memory specificity predicts the course of posttraumatic stress symptomatology. Harvey, Bryant and Dang (1998) showed that reduced specificity on the AMT in the immediate aftermath of a motor vehicle accident (MVA) predicted later posttraumatic stress disorder (PTSD) at follow-up, even when initial acute stress disorder (ASD) diagnosis and depressive symptoms were covaried. Interestingly, however, only reduced specificity of memories with MVA-related content was significantly predictive. Specificity of memories unrelated to the accident was not associated with later PTSD symptoms. This finding, however, was not replicated in a later study using a similar design in a sample of cancer patients (Kangas, Henry, \& Bryant, 2005). Here, although both initial ASD diagnosis and depressive symptoms predicted PTSD six months later, memory specificity (whether cancer-related or not cancer-unrelated) was not a significant predictor. 
What are the reasons for these discrepant results? One possibility is that the relationship between memory specificity and the course of posttraumatic stress is unreliable. Another is that the two studies used trauma populations that differed on critical dimensions. For example, in the MVA survivors the traumatic event itself was over, whereas for many of the cancer victims the problems were presumably chronic and ongoing. Finally, a number of other variables which we know to be related to reduced memory specificity were not always controlled or assessed. For example, Harvey et al. (1998) did not investigate the contributions of trauma severity, history of depression, or executive capacity in their study, all of which can have an impact on reduced specificity (see Williams et al., 2007). This leaves open the possibility that these other variables may be responsible for the reported effects in their study. The first aim of the present study was therefore to examine the replicability of the longitudinal relationship between memory specificity (both trauma-related and unrelated) and later posttraumatic stress, while taking into account for the first time the putative contributions of depression severity, trauma severity and executive functioning.

A key aspect of the studies to date examining the relationship between memory specificity and posttraumatic stress (Moore \& Zoellner, 2007) is that both memory style and symptoms have always been assessed after the trauma. To the best of our knowledge, there are no studies that have assessed memory specificity prior to a traumatic event and then examined symptoms after the event. A potential problem of assessing these two variables post-trauma is that one cannot rule out the possibility that some aspect of the event itself is accounting for both variations in memory specificity and variations in symptoms, as well as the longitudinal relationship between them. Indeed, influential theories of reduced autobiographical memory specificity have argued that it arises as a result of the experience of trauma; for example, as a way of avoiding those specific aspects of the personal past that may be upsetting (Dalgleish, Rolfe, Golden, Dunn \& Barnard, in press; Williams, Stiles, \& Shapiro, 1999; Williams et al., 2007).

However, there are good reasons to believe that pre-trauma memory style may itself influence the course of posttraumatic stress reactions. For example, cognitive theories of posttraumatic stress (see Dalgleish, 2004) suggest that recovery from trauma requires the successful integration of the trauma memory 
into one's autobiographical memory database. An inability to retrieve specific memories could potentially disrupt this process of assimilation. Furthermore, reduced memory specificity is related to reduced social problem solving ability (see Williams et al., 2007), which may be particularly relevant for successfully coping with stressful life events (van Minnen, Wessel, Verhaak, \& Smeenk, 2005).

Of course, assessing autobiographical memory style (or indeed any variable) pre-trauma in a sample where the participants had no knowledge that a traumatic event was likely, is very difficult and would require extremely large samples of unselected participants. However, this research design can be approximated by recruiting a sample for who a traumatic or distressing event is likely in the future but where the event has not yet occurred. The second aim of the present study was therefore to examine, for the first time as far as we are aware, the relationship between memory specificity assessed prior to a predicted, yet nevertheless potentially traumatic, event and posttraumatic stress assessed after that event. To pursue this aim, we recruited a sample of women with pregnancy complications who were hospitalized in anticipation of a difficult and potentially traumatic childbirth experience.

Our rationale for selecting this sample of patients was that previous prospective studies have demonstrated that reduced memory specificity assessed prior to pregnancy-related life-stress predicts later general symptoms of depressed and anxious mood (Mackinger, Loschin, \& Leibetseder, 2000; van Minnen et al., 2005). For example, Mackinger et al. (2000) found that reduced memory specificity to negative word cues during pregnancy was associated with more severe postnatal depressive symptoms three months after normal childbirth.

In order to apply this research design to the study of posttraumatic stress, we recruited women who had just been hospitalized because of an elevated risk of encountering a life-threatening situation during later pregnancy and childbirth and for whom a complicated delivery was anticipated; for example, women with pre-eclampsia. Prior work suggests that up to $30 \%$ of women can present with significant posttraumatic stress, including PTSD, after such pregnancy complications or pregnancy loss (Engelhard, van den Hout, \& Arntz, 2001; Engelhard et al., 2002; see Olde, van der Hart, Kleber, \& van Son, 2006, for a 
review). Furthermore, contrary to Mackinger et al. (2000) and van Minnen et al. (2005) who were more interested in general post-natal depressed and anxious mood, the present study assessed the influence of trauma history, pre-trauma mental health problems, and prepartum emotions and mood (see Williams et al., 2007).

We had three specific hypotheses. First, in order to ground this study within the extant memory specificity literature, we hypothesized that the prepartum data would replicate key established findings. Consequently, we predicted that specific memories at baseline would be negatively related to concurrent depressive symptoms (van Vreeswijk \& de Wilde, 2004) and to any posttraumatic stress arising from prior distressing events (e.g., Schönfeld, Ehlers, Böllinghaus, \& Rief, 2007). We also expected specific memories to be positively related to a measure of executive functioning (Dalgleish et al., 2007). Confirmation of this first hypothesis would serve to validate the AMT for our sample in the prepartum phase.

Our second and central hypothesis was that lower levels of prepartum specific memories would predict higher levels of later postnatal posttraumatic stress symptoms. Critically, we predicted that this relationship would remain after statistical covariation of baseline symptoms and other relevant variables (e.g., trauma severity, executive functioning). As already noted, previous results have indicated that the relationship of retrieved memories to the traumatic or distressing event is particularly important in the prediction of later symptoms (Harvey et al., 1998; van Minnen et al., 2005). Although we were deliberately examining memory specificity pre-trauma, and indeed asked participants to only retrieve memories prior to their hospitalization, we still predicted that a number of the memories generated would be about aspects of the pregnancy prior to hospitalization or about earlier pregnancies, as this is what previous work had indicated (van Minnen et al., 2005). Consequently, for these longitudinal analyses we examined the role of specific memories for pregnancy-related and non-pregnancy-related events separately in our analyses. Furthermore, specific memories to positive and negative cue words were entered separately into the longitudinal analyses because the literature indicates that cue word valence may be important in predicting later symptoms (e.g., Williams et al., 2007). 
Finally, the third hypothesis was that the longitudinal pattern predicted for posttraumatic stress (Hypothesis 2) would be mirrored for depressive symptoms, thus replicating earlier longitudinal findings examining more general mood variables.

\section{Method}

\section{Participants}

Patients hospitalized in the University Hospital Maastricht for pregnancy complications, who were fluent in Dutch, and were physically able to be interviewed (according to hospital staff), received information about this study. Eighty-eight women were eligible to participate in the study. Thirty-four women declined, either because they were only willing to participate at Baseline $(n=5)$, because they were already taking part in other research $(n=5)$, or for an undisclosed reason $(n=24)$. Thus, 54 women completed the Baseline measurement $(61 \%$ response rate).

Nineteen women dropped out of the study between Baseline and Follow-up. Thus, 35 women (mean age $=31.71$ years; $S D=4.84$; mean level of finished education [ranging from 1 - primary school - to 7 - university] $=4.51, S D=1.62$ ) completed both the Baseline measures before delivery and the Follow-up measures six weeks after delivery (65\% retention rate). Reasons for dropping out were that some participants delivered a stillborn child $(n=2)$, were completely occupied by childcare $(n=7)$, or could not be reached or failed to return the measures $(n=10)$. Participants not retained at Follow-up were comparable in age, $F(1,53)=2.95, p<.10$, education, $X^{2}(6)=4.99, p>.50$, and Baseline PSS-SR, IES, and BDI-II scores, FS $<1$, with those retained. However, participants who dropped out at Follow-up tended to be married less frequently than those retained, $\chi^{2}(4)=8.61, p<.08$. The indications for hospital admission were: a hypertensive disorder of pregnancy (i.e., pre-eclampsia; $n=$ 16), pre-term labor $(n=13)$, bleeding in late pregnancy $(n=4)$, unstable diabetes $(n=1)$, and ovarian surgery during pregnancy $(n=1)$. Twelve participants had a history of psychotherapy. Twenty-two women had been pregnant before, with 12 having one $(n=8)$ or more $(n=4)$ children. Ten women had a prior miscarriage, a stillborn child or a child that had died after birth. The present study was part of a larger, ongoing project concerning 
posttraumatic stress after complicated pregnancy. It was approved by the Medical Ethical Committee of the University Hospital Maastricht.

\section{Material}

\section{Autobiographical Memory Test (AMT)}

The AMT contained 5 positive and 5 negative cue words (Wessel, Meeren, Peeters, Arntz, \& Merckelbach, 2001; Williams \& Broadbent, 1986). The AMT cue words in English (Dutch) were: sorry (spijt), happy (gelukkig), angry (boos), safe (veilig), clumsy (onhandig), interested (geïnteresseerd), hurt (gekwetst), successful (succesvol), lonely (eenzaam), and surprised (verrast). Participants were instructed to recall to each cue word a different specific memory about a personally experienced event that happened at a particular time and place before being hospitalized, and that lasted for less than one day. The cue words were orally and visually presented with alternating positive and negative words. To familiarize participants with the procedure, they first practiced with neutral words (e.g., shop, car, trip, conversation) until they understood the task. Participants were required to report a specific memory within $60 \mathrm{~s}$. If participants gave a non-specific answer within that time, they were prompted once with 'Could you be more specific?'. After 60s, the experimenter presented the next cue word. Responses were audiotaped.

The first memories recalled to each cue word were coded by an independent rater. Each response was coded as either a specific memory (i.e., referring to an event at a particular time and place, lasting less than a day), a categoric memory (i.e., involving repeated occasions), an extended memory (i.e., lasting longer than a day), no memory (e.g., an evaluative statement), or an omission (i.e., no response). Furthermore, specific memories were scored as either pregnancy-related or pregnancy-unrelated. A pregnancy-related memory was associated with the current or a previous pregnancy. A second rater scored 250 AMT responses from a subsample of the participants $(n=25)$. Interrater reliability for coding a memory as specific or non-specific was acceptable, kappa $=.70$. 
Beck Depression Inventory - Second edition (BDI-II)

The BDI-II consists of 21 items that each contain four statements that reflect depressive symptoms, in increasing severity (scored 0-3; range 0-63; Beck, Steer, \& Brown, 1996; van der Does, 2002). The BDI-II consists of three subscales that measure somatic (e.g., sleeping problems), cognitive (e.g., feelings of guilt), and affective (e.g., feelings of sadness) symptoms of depression. Participants were asked to complete the Dutch version of the BDI-II (van der Does, 2002) with respect to the previous two weeks. Internal reliability of the BDI-II in the present sample was good at Baseline, Cronbach $a=.87$, and Follow-up, $\mathrm{a}=.84$.

PTSD Symptom Scale - Self Report

The PSS-SR (PSS-SR; Engelhard, Arntz, \& van den Hout, in press; Foa, Riggs, Dancu, \& Rothbaum, 1993) consists of 17 items that correspond to the DSM-IV PTSD symptoms. Items are scored on a 4-point scale $(0=$ not at all, $1=$ once a week, 2 = 2-4 times a week, $3=5$ times or more often; range 0-51) and participants were asked to rate the Dutch version of the scale (Engelhard et al., in press) for the previous month. As with the IES, Baseline ratings were for the previous traumatic event causing the most current distress and Follow-up ratings concerned the pregnancy/birth. Internal reliability was excellent at Baseline, $a=.93$, and Follow-up, $a=.91$.

Impact of Event Scale (IES)

The IES (Brom \& Kleber, 1985; Horowitz, Wilner, \& Alvarez, 1979) was administered to measure the frequency of intrusion and avoidance symptoms of posttraumatic stress within the previous week about an identified negative stressful experience. At Baseline, participants were asked to complete the Dutch version of the IES (Brom \& Kleber, 1985) with respect to whichever previously traumatic/distressing event that is currently bothering them the most, in order to provide a measure of Baseline traumatization. At Follow-up, participants were instructed to complete the IES with respect to the complicated pregnancy and birth. Internal reliability for the IES was excellent at Baseline, $a=$ .91 , and good at Follow-up, $a=.81$. 


\section{Profile of Mood States}

The POMS (POMS; de Groot, 1991; McNair, Lorr, \& Droppleman, 1992) is a widely used instrument to measure subjective mood state. Participants are asked to indicate how much they agree with adjectives describing current mood or feelings on a 5 point scale $(0=$ not at all, $4=$ extremely). Adjectives include annoyed, tensed, and nervous. Higher scores indicate more negative mood. This study used a short Dutch version of the POMS to provide an assessment of current mood (de Groot, 1991; Wald \& Mellenbergh, 1992), and its internal reliability was excellent both at Baseline, $a=.90$, and Follow-up, $a=.92$.

\section{Negative Life-Events Trauma Questionnaire}

The NLETQ (NLETQ; Engelhard, van den Hout, \& Kindt, 2003; Morgan \& Janoff-Bulman, 1994) comprises a list of 33 events (e.g., disease, rape, death of a significant person). Participants indicate which events they have experienced in their lives. Seven items about prior pregnancy complications (e.g., miscarriage, abortion, pre-eclampsia, premature or stillborn child) were used to compute a measure of prior pregnancy trauma.

\section{Raven's Progressive Matrices}

The progressive matrices (Raven, 2000) were used as a measure of executive functioning. Each page consists of a figure that is missing a piece. Participants are asked to choose the correct missing piece from eight alternatives. The test was carried out without time constraints, and the total number of correct answers was computed.

\section{Trauma severity}

The gestational age and birth weight, $r(34)=.85$, were used as an indicator of trauma severity with regard to the pregnancy birth (e.g., Engelhard et al., 2002; Engelhard et al., 2006).

\section{Procedure}

Shortly after admission to the hospital, women were given a brief oral explanation and written information about the study by a nurse. For those who expressed an interest in participating, an appointment was scheduled with a researcher or research assistant who then gave full oral and written information 
about the study. If a woman decided to participate and provided written informed consent, she enrolled in the study. The baseline started with an interview about demographics, prior mental health, pregnancy-related variables, and reasons for being hospitalized. Directly thereafter, the PSS-SR was administered. Here, the woman also indicated on a scale from 0 (not at all) to 3 (very much) how much fear, horror, and helplessness she had experienced during the event reported on the PSS-SR and IES (e.g., Criterion A2 of PTSD; APA, 1994). Then, the NLETQ and Raven's Matrices were administered. After a break, the AMT, POMS, IES and BDI-II were administered. The Follow-up assessment was approximately six weeks after the birth (the birth date was provided by hospital records). At Follow-up, the interview was administered by telephone and focused on the delivery and its aftermath. The PSS-SR, BDI-II, and IES were sent by mail.

\section{Results}

Table 7.1 presents the self-report questionnaire data at Baseline and Follow-up. At Baseline, 24 women completed the PSS-SR and IES in relation to the current hospitalization or pregnancy complications. Three women related symptoms to prior pregnancy experiences, and the remaining eight indicated various experiences, including death or illness of loved ones or work-related problems. Mean scores for fear, horror, and helplessness experienced during the identified stressful event were, $1.85(M d n=2 ; S D=1.18), 0.71(M d n=0 ; S D=1.17)$, and $2.17(M d n=3 ; S D=1.12)$, respectively.

Table 7.1. Means (SDs) of self-reported symptoms of depression (BDI-II), posttraumatic stress (PSS-SR), intrusions and avoidance (IES), and mood (POMS) at Baseline during hospitalization and at Follow-up six weeks after child birth $(\mathrm{N}=35)$.

\begin{tabular}{lcc}
\hline & $\begin{array}{l}\text { Baseline during } \\
\text { hospitalization }\end{array}$ & $\begin{array}{l}\text { Follow-up } 6 \text { weeks after } \\
\text { child birth }\end{array}$ \\
\hline PSS-SR & $14.65(11.79)$ & $7.64(6.45)$ \\
IES total & $18.31(15.03)$ & $8.21(8.92)$ \\
IES intrusion subscale & $11.49(9.49)$ & $5.03(5.41)$ \\
IES avoidance subscale & $6.83(7.38)$ & $3.18(4.81)$ \\
BDI-II & $12.31(7.73)$ & $8.64(6.17)$ \\
BDI-II somatic symptoms & $8.54(4.20)$ & $5.88(3.57)$ \\
BDI-II affective symptoms & $1.53(2.06)$ & $.88(1.29)$ \\
BDI-II cognitive symptoms & $2.09(2.74)$ & $1.79(2.39)$ \\
POMS & $27.83(16.27)$ & $29.63(16.11)$ \\
\hline
\end{tabular}

Note. BDI-II = Beck Depression Inventory; PSS-SR = Posttraumatic Symptoms Scale - Self-Report; IES = Impact of Event Scale; POMS = Profile of Mood States. 
These data were similar for the subgroups of women who did versus did not rate the PSS-SR and IES with respect to being hospitalized or pregnancy complications, ts $<1$.

\section{Characteristics related to giving birth}

Twenty-two women had a Caesarean birth, eight women delivered after being medically induced, and five women had a normal childbirth. Mean hospital stay during pregnancy was 18.34 days ( $S D=13.50$; Range: $2-56$ days), with a delivery at a mean gestational age of 35.43 weeks ( $S D=3.66$; Range: 28.40 42.00 weeks), and a mean birth weight of 2402.49 grams ( $S D=880.58$; Range: $740-4340$ grams). A delivery before 37 weeks is called premature (e.g., Steer, 2005) and a normal birth weight is between 2500-4500 grams (e.g., Frederick, Williams, Sales, Martin, \& Killien, 2007).

Table 7.2. Mean (SDs) numbers of pregnancy-related and -unrelated specific memories, and general categoric memories, to positive and negative cue words on the Autobiographical Memory Test (AMT) at Baseline $(\mathrm{N}=35)$.

\begin{tabular}{lccl}
\hline & Positive cue & Negative cue & Total \\
\hline AMT-S & $3.23(1.40)$ & $2.31(1.59)$ & $5.54(2.73)$ \\
AMT-NPR & $2.00(1.46)$ & $1.66(1.45)$ & $3.66(2.54)$ \\
AMT-PR & $1.23(1.14)$ & $.66(1.03)$ & $1.89(1.66)$ \\
AMT-GC & $.60(.81)$ & $.63(.91)$ & $1.23(1.42)$ \\
\hline
\end{tabular}

Note. AMT-S = number of specific memories; AMT-NPR = specific memories unrelated to pregnancy; AMT-PR = specific memories related to pregnancy; AMT-GC = categoric memories. The mean (SD) numbers of extended memories, no memories, and omissions were 1.74 (1.52), .57 (1.01), and .66 (.91), respectively.

Hypothesis 1: Autobiographical memory specificity is negatively correlated with concurrent depressive and posttraumatic stress symptoms and positively correlated with executive functioning

Table 7.2 presents the data for the different autobiographical memory specificity variables. Correlation analyses showed that total numbers of specific memories $(d . f .=33)$ were negatively correlated with scores on the BDI-II, $r=-.31, p=.08$, PSS-SR, $r=-.48, p=.004$, IES total, $r=-.44, p=.008$, IES intrusion, $r=-.41, p$ $=.02$, and IES avoidance, $r=-.38, p=.03$, and positively correlated with scores 
on Raven's Matrices, our measure of executive functioning, $r=.47, p=.005$. Overall, these results support Hypothesis $1^{19}$.

Hypotheses 2 and 3: Autobiographical memory specificity predicts later posttraumatic stress and depression symptoms

To examine Hypotheses 2 and 3 we first computed zero-order correlations between our various Baseline variables and the relevant Follow-up outcome measures (PSS-SR, IES, BDI-II; see Table 7.3). For each outcome measure, we next examined a regression model including just the four AMT variables as predictors (i.e., specific pregnancy-related memories to positive [AMT-PR-pos] and negative [AMT-PR-neg] cues, and specific pregnancy-unrelated memories to positive [AMT-NPR-pos] and negative [AMT-NPR-neg] cues) to examine which aspects of memory specificity, if any, independently accounted for variance in the outcome measures. Finally, for each outcome measure we repeated this first regression this time with the relevant mood and symptom variables at Baseline entered on a first step, along with any demographic or trauma-related variables that had shown a significant zero-order correlation with the outcome variable. The four AMT variables were entered on a second step to investigate which aspects of memory specificity, if any, accounted for significant variance in the outcome variable after covarying for Baseline mood and other significant zero-order predictors.

For all regressions we verified that the variables were not significantly collinear.

PSS-SR data

The Follow-up PSS-SR data contained one outlier, which was truncated into a value that was one point higher than the second highest value (Tabachnick \& Fidell, 2007). The mean level of posttraumatic stress as indicated by the PSSSR (Range: 0-20) at Follow-up was below previously reported clinical cut-off scores (e.g., cut-off < 14, Coffey, Gudmundsdottir, Beck, Paylo, \& Miller, 2006; see also Brewin, 2005).

\footnotetext{
19 The pattern of results was similar when correlations were computed on the basis of all 54 women who completed the Baseline measurement, rs (52) $>$ I.25I, ps $<.08$.
} 
Table 7.3. Zero-order correlations of Follow-up posttraumatic stress and depressive symptoms (top row), with Baseline depressive and posttraumatic symptoms as well as with prior (pregnancy) trauma, prior therapy, birth weight and measures of autobiographical memory specificity (first column) $(\mathrm{N}=35)$.

\begin{tabular}{llll}
\hline & PSS-SR & IES & BDI-II \\
\hline Raven accuracy & -.29 & $-.48^{\star \star}$ & -.29 \\
BDI-II & $.36^{\star}$ & .12 & $.37^{\star}$ \\
PSS-SR & $.50^{\star *}$ & .23 & .27 \\
IES total & $.45^{\star \star}$ & $.33^{\star}$ & $.46^{\star \star}$ \\
IES avoidance & .31 & $.42^{\star}$ & $.34^{\star}$ \\
IES intrusions & $.46^{\star \star}$ & .19 & $.42^{\star}$ \\
POMS & .29 & .09 & .30 \\
Prior therapy (psychologist or psychiatrist) & -.17 & -.33 & .02 \\
Prior trauma (NLETQ) & .08 & .18 & .19 \\
Prior pregnancy trauma (NLETQ) & .21 & .29 & .20 \\
Birth weight & $-.39^{\star}$ & -.32 & -.29 \\
AMT-S-positive & $-.38^{\star}$ & -.32 & $-.39^{\star}$ \\
AMT-S-negative & -.23 & $-.35^{\star}$ & -.17 \\
AMT-PR-positive & $-.38^{\star}$ & $-.34^{*}$ & $-.57^{\star \star}$ \\
AMT-PR-negative & -.02 & .02 & -.18 \\
AMT-NPR-positive & -.07 & -.04 & .07 \\
AMT-NPR-negative & -.26 & $-.39^{\star}$ & -.15 \\
\hline
\end{tabular}

Note. ${ }^{*}<.05 ;{ }^{*}<.01$; BDI-II $=$ Beck Depression Inventory-II; PSS-SR = Posttraumatic Symptoms Scale Self-Report; IES = Impact of Event Scale; POMS = Profile of Mood States; NLETQ = Negative Life-Events Trauma Questionnaire; AMT-S = specific memories; AMT-S-positive = specific memories to positive cues; AMT-S-negative = specific memories to negative cues; AMT-NPRpositive $=$ specific memories pregnancy-unrelated to positive cues; AMT-NPR-negative $=$ specific memories pregnancy-unrelated to negative cues; AMT-PR-positive = specific memories pregnancyrelated to positive cues; AMT-PR-negative = specific memories pregnancy-related to negative cues.

Table 7.3 presents the zero-order correlations between Baseline and Follow-up measures. As can be seen, BDI-II, PSS-SR and IES scores at Baseline, birth weight, and the total number of specific memories (AMT-S) along with the number of pregnancy-related specific memories (AMT-PR) to positive cues were all significantly correlated with Follow-up PSS-SR. The first regression model, with PSS-SR scores at Follow-up as the dependent variable and the four AMT scores as predictors was significant, $F(4,30)=2.91, p=.04, R^{2}=.28$, indicating that total reduced memory specificity was related to higher subsequent posttraumatic stress. AMT-PR-pos scores were the only significant independent predictor, $\beta=-.63, t=2.89, p=.007$, indicating that reduced specificity for positive pregnancy-related memories was associated with greater later posttraumatic stress. Other $\beta s<1.291$, ts $<1.40$, $p s>.18$.

For the second regression we entered Baseline PSS-SR and BDI-II, along with birth weight on Step 1, and the four AMT variables on Step 2. Step 1 was 
significant, $F(3,30)=7.23, p=.001, R^{2}=.42$. Step 2 accounted for incremental variance in PSS-SR scores, $F_{\text {change }}(7,26)=4.76, p=.001, R_{\text {change }}^{2}=.14$, indicating that reduced total specific memories was associated with later greater posttraumatic stress even after covarying the relevant Baseline measures. As with the first regression, reduced specificity of positive pregnancy-related memories (AMT-PR-pos) was the only significant independent predictor of higher later post-traumatic stress, $\beta=-.46, t=2.31, p=.03$. Other $\beta \mathrm{s}<1.29 \mathrm{I}$, ts $<1.60$, ps $>$.12.

IES data

The mean level of posttraumatic stress as indicated by the IES-total (Range: 034) at Follow-up was also below previously reported clinical cut-off scores (e.g., cut-off < 19, Wohlfarth et al., 2003; see also Brewin, 2005). As can be seen from Table 7.3, Follow-up IES scores correlated with a number of the AMT specificity variables at Baseline, along with the Baseline IES scores and scores on Raven's Matrices, the measure of executive functioning. The regression examining the predictive value of the four AMT variables was significant $F$ (4, $30)=3.00, p=.04, R^{2}=.28$, indicating again that reduced total specific memories was associated with later posttraumatic stress. There were trends for AMT-PR-pos, $\beta=-.40, t=1.87, p=.07$, and AMT-NPR-neg, $\beta=-.36, t=1.74$, $p=.09$, to be independent predictors indicating that reduced specificity of positive pregnancy memories and greater specificity of non-pregnancy-related negative memories tended to predict later, higher levels of posttraumatic stress. Other $\beta s<.12$, ts $<1, p s>.50$. However, for the follow-up regression with Baseline IES-total, BDI-II and Raven's Matrices scores on Step 1 and the AMT variables on Step 2, Step 1 was significant, $F(3,29)=4.67, p<.01$. However, Step 2 did not account for significant incremental variance, $F_{\text {change }}(7,25)<1$, providing no support for the hypothesis that memory specificity would account for variance in Follow-up IES scores once variance accounted for by the other relevant baseline variables was taken into account.

$B D I-I /$ data

The mean level of depressive symptoms as indicated by the BDI-II (Range: 021 ) at Follow-up was in the normal range of ups and downs (BDI-Il scores < 9; e.g., Beck et al., 1996). Table 7.3 shows that Follow-up BDI-II scores correlated 
with a number of Baseline AMT variables as well as Baseline BDI-II and IES scores. The regression model with the four AMT predictors was significant, $F$ (4, 29) $=4.76, p=.004, R^{2}=.40$, indicating once more that reduced total AMT specificity was associated with higher later levels of depression symptoms. Once again, AMT-PR-pos was the only significant independent predictor, $\beta=-$ .83, $t=4.04, p<.001$, though there was a trend in the case of AMT-PR-neg, $\beta$ $=-.33, t=1.84, p=.08$, indicating that reduced specificity in both positive and negative (trend) pregnancy-related memories was associated with higher levels of subsequent depressive symptoms. Other $\beta s<\mid .35 \mathrm{l}$, $t \mathrm{~s}<1.65$, $p s>.12^{20}$.

In the second regression with baseline PSS-SR, IES and BDI-II scores on Step 1 and the AMT variables on Step 2, Step 1 was significant, $F(3,29)=4.17, p=$ $.01, R^{2}=.30$, and Step 2 accounted for significant incremental variance in BDIII, $F_{\text {change }}(7,25)=3.37, p=.01, R_{\text {change }}^{2}=.19$, indicating that reduced total specific memories was associated with later greater depressive symptoms even after covarying the relevant Baseline measures. As with the first regression, reduced specificity of positive pregnancy-related memories (AMT-PR-pos) was the only significant independent predictor of higher later depression scores, $\beta=$ -.65, $t=2.29, p=.03$. Other $\beta \mathrm{s}<1.231$, $t \mathrm{~s}<1^{21}$.

\section{Discussion}

The main aim of this study was to investigate whether autobiographical memory specificity, assessed pre-trauma, would independently predict levels of posttraumatic stress, assessed post-trauma, in a sample of pregnant women admitted to hospital in advance of anticipated late-pregnancy and delivery complications. In line with prior research (e.g., Williams et al., 2007), the data

\footnotetext{
${ }^{20}$ Somatic symptoms (e.g., lack of sleep, loss of concentration) may be related to late pregnancy and postpartum rather than to a mood disturbance. Therefore, we repeated this regression using only the cognitive and affective items of the BDI-II. The model remained significant $F(4,29)=3.13$, $p=.03, R^{2}=.31$, with for AMT-PR-pos as the only significant independent predictor, $\beta=-.72, t=$ 3.26, $p=.003$, with a trend for AMT-NPR-neg, $\beta=-.35, t=1.80, p=.08$.

${ }^{21}$ We again repeated this regression using only the cognitive and affective items of the BDI-II. The pattern of results was the same with the AMT variables on Step 2 accounting for significant incremental variance in BDI-II scores, $F_{\text {change }}(7,25)=2.50, p=.04, R_{\text {change }}^{2}=.16$ and with AMT-PRpos scores as the only significant independent predictor, $\beta=-.75, t=2.37, p=.03$.
} 
supported our first hypothesis that prepartum memory specificity would be negatively related to concurrent symptoms of depression and post-traumatic stress, and positively related to a measure of executive functioning, thus validating the AMT for this sample at the Baseline assessment.

The data also confirmed our second and principal hypothesis, demonstrating for the first time to our knowledge that reduced pre-trauma memory specificity predicted greater, later posttraumatic stress symptoms (on the PSS-SR). Importantly, this effect remained significant after covarying out baseline symptoms. Interestingly, this predictive relationship was primarily accounted for by the number of specific memories to positive cues that were thematically related to the pregnancy. In other words, women who recalled fewer specific pregnancy-related memories to positive cues before their complicated delivery were more likely to have increased levels of posttraumatic stress afterwards. These results extend, to a pre-/post-trauma design, the findings of Harvey et al. (1998) who showed that fewer specific accident-related memories produced on the AMT in the first few weeks after an MVA predicted posttraumatic stress severity six months later. The current data demonstrate this longitudinal relationship after taking into account a range of other variables previously associated with reduced memory specificity, including prior trauma, history of psychopathology and executive functioning (Williams et al., 2007).

Similar analyses in the present study using the IES, as opposed to the PSS-SR as the outcome variable, also revealed that reduced memory specificity predicted higher later levels of posttraumatic stress. However, these findings were no longer significant once the influence of Baseline symptoms was covaried, although the findings were in the expected direction. Finally, we confirmed our third hypothesis that fewer specific memories would independently predict an increase in later depressive symptoms, thus replicating and extending previous longitudinal findings examining the prediction of depressed mood (see Williams et al., 2007).

The data raise a number of interesting questions. First, why did the predictive effects pertain exclusively to reduced pregnancy-related specific memories to positive cues? One possible explanation is that a relative inability to retrieve positive pregnancy-related memories might have hindered the expectancy of or 
belief in a positive outcome to the pregnancy, thus making it more difficult to cope with any complications (van Minnen et al., 2005). Similarly, we know that reduced memory specificity is related to poorer social-problem solving and it is possible that relatively impaired access to positive pregnancy-related personal material may have compromised functional problem-solving around issues relating to difficulties later in the pregnancy and postnatal period (Williams et al., 2007).

A second issue is why we found such clear predictive effects for posttraumatic stress measure done with the PSS-SR, with less convincing effects on the IES. One possibility is that the PSS covers a greater time period (one month) than the IES (one week). Consequently, responses on the PSS at six weeks postpartum would have captured the greater part of the participant's posttraumatic stress response whereas this may not have been the case for the IES. Furthermore, the IES only assesses symptom frequency and not severity and it may be that independent relationships between posttraumatic stress and memory specificity involve severity of symptoms.

The present study was a preliminary investigation and thus is not without its limitations. First, although the study design moved close to a genuine preversus post-trauma methodology, the participants were nevertheless aware that potentially traumatic experiences were imminent as they had recently been hospitalized in anticipation of such eventualities. One could therefore argue that the 'trauma phase' had already started. However, we took care to ask participants at Baseline to generate memories from prior to their hospitalization, and to assess posttraumatic stress at Follow-up for the difficulties and problems that occurred after this Baseline assessment. In other words, memory assessment was carried out before the event that led to the later symptoms of posttraumatic distress that we assessed.

Secondly, we did not assess premorbid psychiatric difficulties, nor posttraumatic stress, using structured clinical interviews, relying instead on self-report questionnaire measures. We were therefore not able to derive formal psychiatric diagnoses such as major depressive disorder or PTSD. That said, the data on memory specificity and posttraumatic stress are strikingly similar for casecontrol studies (e.g., PTSD versus no-PTSD) and correlational studies using 
variation on self-report questionnaire measures (see Moore \& Zoellner, 2007), so this seems less of a concern for the current study. Third, although an objective measure of trauma severity (i.e. birth weight, Engelhard et al., 2002; 2006) was used and most women reported intense fear and helplessness related to the childbirth, we did not directly measure whether the delivery itself was a Criterion A trauma according to the DSM-IV (APA, 1994).

In conclusion, the present study showed that retrieval of fewer specific pregnancy-related autobiographical memories to positive cue words during complicated pregnancy predicted posttraumatic stress symptoms and depressive symptoms six weeks postpartum. This relation remained significant after taking into account variables commonly related to both autobiographical memory specificity and symptoms. The data indicate, for the first time, that memory specificity assessed in advance of a potentially traumatic event, can independently predict posttraumatic stress symptoms surrounding that event at a later juncture. 
General discussion and conclusion 
The research described in the present thesis aimed to explore how the positive relationship between overgeneral memory and intrusions (e.g., Kuyken \& Brewin, 1995) can be accounted for. To start with, the first study explored whether the association between intrusions, avoidance, and overgeneral memory that is generally found in clinical samples could be reproduced in a non-clinical sample. Subsequently, we investigated in five experiments whether retrieval-induced forgetting would be a fruitful experimental analogue to explain the co-occurrence of overgeneral memory and intrusions (e.g., Wessel, Merckelbach, \& Dekkers, 2002).

In the subsequent studies, hypotheses derived from the Carfax model were explored (Williams, 2006; Williams et al., 2007). First, we investigated whether both overgeneral memory and intrusions would be the result of functional avoidance (Williams, 2006). Second, we tested the hypothesis that overgeneral memory and specific intrusions would be the result of two different retrieval routes (e.g., Conway, Singer, \& Tagini, 2004; Williams et al., 2007). One being the result of a hierarchical generative search process (generative retrieval) and the other relying on direct association between the retrieval cue and the memory (direct retrieval). The final study examined whether pre-trauma assessed overgeneral memory would be a predictor to develop later posttraumatic stress symptomatology.

\section{Summary of empirical findings}

Prior research has shown a significant association of overgeneral memory with intrusions and avoidance symptoms in clinical samples of depressed and/or traumatized individuals (e.g., Kuyken \& Brewin, 1995; Schönfeld \& Ehlers, 2006). Other research has suggested that intrusions (Berntsen, 2001), a tendency to avoid unwanted memories (Wenzlaff \& Wegner, 2000), and a relative lack of specificity (Merckelbach, Muris, \& Horselenberg, 1996) also occur in non-clinical samples, although sample sizes in these studies were sometimes relatively small (e.g., Wenzlaff \& Wegner, 2000). Therefore, the first study described in Chapter 2 explored whether the overgeneral memoryintrusion link could be reproduced in a student sample. Whereas we found no significant correlation between overgeneral memory and self-reported intrusions, the results did reveal a positive association between avoidance 
symptoms and categoric memories to negative cues. This association remained significant after controlling for depressive symptoms and recency of negative life events that participants described. Thus, we were able to replicate individual differences on the AMT in a student sample.

The two studies described in Chapter 3 explored whether retrieval-induced forgetting would occur within the domain of autobiographical memories. Usually, overgeneral memory is measured with the AMT using a broad range of retrieval cues. In contrast, the retrieval practice paradigm typically uses relatively narrow categories of retrieval cues (e.g., Anderson, Bjork, \& Bjork, 1994; Barnier, Hung, \& Conway, 2004). Therefore, in the first study, the retrieval practice paradigm was applied to relatively broad categories of positive and negative autobiographical memories. The results, indeed, demonstrate a retrievalinduced forgetting effect, replicating Barnier et al.'s (2004) findings. As the effect was relatively small $(3 \%)$, a second study was performed with only negative autobiographical memories. The data, again, show a retrieval-induced forgetting effect, but this time larger (11\%) than in the first study. The data from this second study further revealed that part of the retrieval-induced forgetting effect was caused by a non-inhibitory phenomenon because some of the unrehearsed memories were reported in the incorrect category at cued recall. This suggests that, these memories have not become less available (e.g., Anderson, 2003), but were recalled in an incorrect context.

The three studies reported in Chapter 4 further investigated the merits of retrieval-induced forgetting within emotionally and negatively valenced slides. The studies were inspired by the attentional narrowing effect (e.g., Reisberg \& Heuer, 2004) and by the fact that intrusions contain perceptual and sensoric details (e.g., Conway et al., 2004). Rather than looking at the effect of repeated retrieval of memories on other memories, here, the effect of some details on unrehearsed details of the same emotional scene was investigated. None of the studies supported the hypothesis that repeated retrieval of central or peripheral details of emotional slides would deteriorate the recall of unpracticed peripheral and central details of that same slide. However, participants who repeatedly practiced central details (study 1 and 3 ) made more commission errors in unpracticed details during free recall. Thus, none of the three experiments 
showed data in support of a retrieval-induced forgetting account within the context of memory for emotionally and negatively valenced slides.

In Chapter 5, we examined in a sample of non-clinical participants whether active thought suppression (e.g., Ehlers \& Clark, 2000; Wenzlaff \& Wegner, 2000) of ones most negative experience would elicit more intrusions about this experience, on the one hand and subsequently, fewer specific memories on an AMT, on the other hand. The negative condition was compared to a condition in which a memory of a neutral experience was suppressed. The results demonstrated that, independent of suppression instruction or valence of the experience, individuals who reported more intrusions about the critical event during the AMT also displayed larger reductions in specific memory retrieval. Furthermore, there was a tendency in the data showing that those individuals, who suppressed thoughts about their most negative event, experienced a less negative mood than those individuals, who did not suppress their negative event (e.g., Williams, Stiles, \& Shapiro, 1999).

Chapter 6 described an experiment that examined whether overgeneral memory and specific intrusions rely on different retrieval processes (e.g., Conway et al., 2004; Williams et al., 2007). The idea was that everyday intrusions rely on direct associations between retrieval cues and specific memories (direct retrieval), whereas memories retrieved on the AMT are the result of an iterative retrieval cycles in a hierarchical system (generative retrieval). Two different versions of the AMT were used to test this hypothesis in a sample of individuals with a history of childhood sexual abuse (CSA) and control participants. The data supported the hypothesis that the CSA participants were more overgeneral on the standard AMT, but that this difference disappeared on the AMT with direct retrieval cues. This effect was stronger for CSA participants with more posttraumatic stress symptoms. Furthermore, remained significant when participants with past or current major depressive disorder were excluded, and when only those participants with corroborated CSA were included.

In Chapter 7, we described a prospective study that examined whether pretrauma reduced memory specificity would predict the development of posttraumatic stress and depressive symptoms after experiencing a life-stress event (e.g., Harvey, Bryant, \& Dang, 1998; Mackinger, Loschin, \& Leibetseder, 
2000; van Minnen, Wessel, Smeenk, \& Verhaak, 2005). To this end, women with pregnancy complications were assessed directly after their hospitalization (prepartum) and six weeks after giving birth. The results revealed that women, who retrieved fewer specific memories with a pregnancy-related content in response to positive cues, reported more posttraumatic stress and depressive symptoms six weeks after giving birth. This effect remained significant after covarying relevant variables that were both related to baseline autobiographical memory specificity and follow-up posttraumatic stress and depressive symptoms.

\section{Underlying mechanism of overgeneral memory and intrusions}

\section{Retrieval-induced forgetting}

What do the present data say about retrieval-induced forgetting as an experimental analogue for the association between overgeneral memory and intrusions? Are there indications that an inhibitory process may be the underlying mechanism? First, the data of Chapter 5 demonstrate retrievalinduced forgetting effects with emotional autobiographical memories. Retrospectively, however, one might question whether we really modeled intrusions vs. overgeneral memory. For example, whereas repeated retrieval within the retrieval practice paradigm was voluntary, intrusive recollections have an involuntary and unwanted character (APA, 1994; Ehlers \& Clark, 2000). Germane to this issue is Williams' (2006) suggestion that retrieval of posttraumatic intrusions is perhaps not the closest analogue that we were trying to model, but rather ruminative processing (e.g., Watkins \& Teasdale, 2001). In contrast to intrusive memories, in rumination, depressed or traumatized individuals voluntarily come up with past experiences trying to explain why they feel terrible (Williams, 2006; see also Speckens, Ehlers, Hackmann, Ruths, \& Clark, 2007 for a comparison of intrusions and rumination within PTSD).

The suggestion of Williams (2006) sounds promising. The voluntary/involuntary issue is no longer an obstacle and it gives new directions to future research. Yet, this suggestion is also not without constraints. The retrieval-induced forgetting effect pertains to memories and is based on the assumption that repeated retrieval of some memories reduces the availability of unrehearsed memories that share similar retrieval cues (e.g., Anderson, 2003; Barnier et al., 
2004; Chapter 3). Rumination, however, is usually not about memories, but about abstract and verbal cognitions (e.g., related to failure or other negative self schemas, Watkins \& Teasdale, 2001; 2004). Even though a recent study (e.g., Storm, Bjork, Bjork, \& Nestojko, 2006) has demonstrated that the act of repeated retrieval with the absence of a retrieval practice effect is sufficient to obtain a retrieval-induced forgetting effect, the question arises whether retrieval practice of abstract and verbal cognitions induces a similar effect. This is an empirical question that remains open until further experimentation.

Second, the studies in Chapter 4 do not extend retrieval-induced forgetting to details of emotionally negative slides. This finding is also not in favor of a retrieval-induced forgetting analogue to explain the co-occurrence of intrusions and overgeneral memory. It should be noted, though, that the idea behind this study was somewhat different. The rationale was based on the attentional narrowing literature (e.g., Reisberg \& Heuer, 2004) and the question of interest was whether the retrieval-induced forgetting effect could be found in other PTSD-like memory phenomena, such as the phenomenon that some psychologists termed psychogenic amnesia (e.g., Holmes et al., 2005). To this end, instead of looking at the effect of repeated retrieval of some memories on other memories, the focus here was on the effect of repeated retrieval of details on unrehearsed details within one scene.

In a typical retrieval-practice paradigm, study trials are presented separately in a series of category-exemplar pairs (e.g., Anderson et al., 1994), whereas, here, presentation of category-exemplars was necessarily simultaneously due to the nature of the material. Furthermore, there was an incidental learning instruction, instead of the typically used intentional instruction. Possibly, forming explicit (episodic) cue-exemplar associations may be crucial to elicit a retrieval-induced forgetting effect (Perfect et al., 2004; Racsmány \& Conway, 2005). Such explicit associations were not formed during the present experiments, which may have prevented a typical retrieval-induced forgetting effect from occurring. The data of Chapter 4, therefore, suggest that retrieval-induced forgetting is difficult to generalize to complex emotionally negative visual material, and to traumatic memories for that matter. 
Even if retrieval-induced forgetting might play a role in the link between intrusions/rumination and overgeneral memory, it seems relatively implausible that an inhibitory mechanism making the unrehearsed memories less available is solely responsible for this link (Anderson, 2003). The retrieval-induced forgetting effect in Chapter 3 was partly due to the fact that some of the unrehearsed memories were actually recalled, but in response to other retrieval cues. This means that participants were still able to retrieve unrehearsed memories, though not in the right context (i.e., these memories have not become less available). The remaining unrehearsed memories that were part of the retrieval-induced forgetting effect might have become less available and inhibition might have played a role. However, this was a small effect and its relevance in real life may be questioned. Furthermore, it should be noted that it is relatively difficult to directly measure inhibition (e.g., independent cueing, Anderson \& Spellman, 1995), specifically in more ecologically valid material in which it is extra complex to control for boundary conditions of retrieval-induced forgetting.

What is more, the findings reported in Chapter 6 are difficult to reconcile with an inhibitory explanation (e.g., Anderson, 2003). Individuals with a history of CSA retrieved considerably more specific memories on the AMT version with direct retrieval cues than on the standard AMT. Furthermore, they were able to retrieve an equal number of specific memories on the direct AMT as compared to the control participants. If an inhibitory mechanism would underlie overgeneral memory, then CSA participants should also have been less specific than the controls on the direct AMT because their memories would have become less available. However, this was not the case: when cued with different - concrete and highly imageable - retrieval cues, individuals with CSA were able to retrieve an equal number of specific autobiographical memories as the control participants.

To sum up, the data of the current thesis have shown that retrieval-induced forgetting is unlikely to be the primary account to explain the association between intrusions and overgeneral memory. Possibly, retrieval-induced forgetting may play a role in the relation between rumination and autobiographical memory retrieval. Future research may reveal whether this is indeed a fruitful suggestion to follow. 


\section{Carfax model}

As described in the introduction, the capture and rumination part of the Carfax model is silent about explaining the co-occurrence of intrusions and overgeneral memory. Furthermore, in the previous section, we already discussed that retrieval-induced forgetting might be an interesting avenue for future research on rumination and overgeneral memory (Williams, 2006). Therefore, the next sections focus on whether the current data may be explained by affect regulation and reduced executive capacity.

\section{Functional avoidance}

Concurring with prior research on avoidance and overgeneral memory (e.g., Hermans, Defranc, Raes, Williams, \& Eelen, 2005), the association between avoidance symptoms and overgeneral memory reported in Chapter 2 concurs with the idea that overgeneral memory retrieval itself may be an avoidant coping style to reduce negative affect related to traumatic events (e.g., Williams et al., 2007). The tentative data in Chapter 5 showing that suppression of ones most negative experience diminished negative mood add to this idea. However, Chapter 5 also demonstrates that both emotionally negative and neutral intrusions - independently of the thought suppression instruction - were related to reduced autobiographical memory specificity. This is in contradiction with the functional avoidance account for two reasons. First, it hypothesizes that only emotionally negative intrusions are related to reduced autobiographical memory specificity, whereas the present data show that intrusions about neutral experiences are also related to reduced memory specificity. Second, and possibly more important, the data in Chapter 5 show an overall negative correlation of intrusions with autobiographical memory specificity, indicating that suppression did not play a role in the relation between intrusions and overgeneral memory.

In addition, the findings reported in Chapter 6 imply that overgeneral memory retrieval as a way to avoid distressing specific autobiographical memories fails to address one of its primary sources: participants displaying an overgeneral memory also show intrusive trauma memories. The data of Chapter 6 suggest that traumatized individuals seem to have an intact direct retrieval route and intrusions are most likely recalled via this way. As a result, overgeneral memory 
retrieval via the generative retrieval route does not prevent intrusions from entering consciousness when they are retrieved via the direct retrieval route. Furthermore, Golden, Dalgleish and Mackintosh (in press) showed that participants with complicated grief retrieved more specific memories on a Biographical Memory Task (BMT) about the deceased person than on a BMT about a loved person that was still alive or on a standard AMT. These data suggest that also during generative retrieval traumatized participants are still deliberately retrieving distressing memories. The combination of these data and those of Chapter 5 and 6 would not entirely reject functional avoidance as an explanation for intrusions and overgeneral memory. It does suggest that it is imperfect.

In summary, functional avoidance may account for the co-occurrence of specific intrusions and overgeneral memory to some extent (see also Williams, 2006). However, traumatized individuals still retrieve - deliberately or involuntarily distressing memories about their traumatic experiences (Golden et al., in press; Chapter 6) and it remains unclear how such a functional avoidant strategy would exactly work. Furthermore, the data of Chapter 5 suggest that avoidance does not underlie the o-occurrence of intrusions and reduced autobiographical memory specificity. Williams (2006) and Raes, Hermans, Williams, and Eelen (2006) argue that an avoidant coping style may be efficient in the short run, but that it becomes dysfunctional in the long run. So far, there is limited evidence for this view (e.g., Raes et al., 2003; 2006). Therefore, at least additional research is recommended that focuses on how exactly functional avoidance in the context of overgeneral memory would work. Nevertheless, other explanations may be equally or more likely to explain the relationship between overgeneral memory and intrusions.

\section{Reduced executive capacity}

There are some indications in the current data that reduced executive capacity may explain the co-occurrence of intrusions and overgeneral autobiographical memory retrieval (Dalgleish et al., 2007; Williams et al., 2007). The findings of Chapter 7 show a positive association between a measure of executive functioning - Raven Progressive Matrices - and specific memory retrieval (e.g., Dalgleish et al., 2007). Although this relationship is purely correlational, it does 
indicate that autobiographical memory retrieval on a standard AMT (e.g., Williams \& Broadbent, 1986) requires a certain amount of cognitive capacity (e.g., Dalgleish et al., 2007; Williams et al., 2007). Approached from a different angle, the findings of Chapter 6 also agree with this view. Whereas this was not directly tested, the fact that CSA individuals were able to retrieve specific memories on an AMT with direct cues, but not on the standard AMT might imply that more cognitive (executive) capacity is needed for the latter. Of course, this interpretation remains open to empirical testing. In agreement with this interpretation, Williams et al. (1999) found that performance on a standard AMT suffered from a dual task, whereas performance on an AMT with more direct cues did not.

Furthermore, while functional avoidance cannot account for the fact that both negative and neutral intrusions about suppressed and non-suppressed critical autobiographical memories are related to reduced memory specificity in Chapter 5, reduced executive capacity may explain this finding (e.g., Dalgleish et al., 2007). Possibly, intrusions during autobiographical memory retrieval on the AMT may have consumed limited working memory capacity that subsequently harmed the retrieval of specific autobiographical memories. Thus, due to the intrusions popping up during the AMT, there might have been not enough executive capacity anymore to search for specific memories. Another possibility might be that there were some participants who had a reduced working memory capacity as a trait and were, therefore, less able to inhibit the involuntary intrusions during autobiographical memory retrieval on the AMT.

These (post-hoc) hypotheses were not empirically tested in the study described in Chapter 5 and are matter for subsequent experiments. Nevertheless, a reduced executive capacity account seems able to explain the current findings that are relatively difficult to unite with functional avoidance. In future, it might be interesting to design experiments that are able to discriminate between reduced executive capacity and functional avoidance as possible underlying mechanisms of overgeneral memory.

\section{Two retrieval routes}

Regardless of which processes underlie overgeneral autobiographical memory retrieval in traumatized or depressed individuals (e.g., functional avoidance, 
reduced executive capacity, rumination), the findings of Chapter 6 show that processes that disrupt generative retrieval seem to have little or no effect on direct retrieval of autobiographical memories. That is to say, CSA individuals who reported intrusions about their traumatic experience and showed reduced memory specificity on a standard AMT, were able to retrieve more specific autobiographical memories on a concrete AMT. This effect was even more pronounced in those participants with CSA who demonstrated more PTSD symptomatology (e.g., intrusions). These results agree with prior experimental work in healthy samples showing that more specific memories were retrieved to highly imageable cue words (Williams et al., 1996; 1999) and with data of Schönfeld and Ehlers (2006) indicating that PTSD patients retrieve more specific autobiographical memories to picture cues than to word cues.

The data described in Chapter 6 further concur with the theoretical accounts of Conway et al. (2004) and Williams et al. (2007) that assume relatively distinct retrieval processes operating under different circumstances. They are also in accordance with other theories about trauma and PTSD that suggest dual processes or representations (e.g., Brewin, Dalgleish, \& Joseph, 1996; Foa \& Kozak, 1986; Janoff-Bulman, 1992; see also Dalgleish, 2004). For example, in their dual representation theory, Brewin et al. (1996) argue that there are two kinds of representations involved in the memory of traumatic experiences. The first type of representations consists of the conscious experience of a traumatic event and is termed verbally accessible memory (VAM). VAMs can be deliberately accessed, can be edited by the individual, and are completely contextualized in the autobiographical memory (e.g., generative retrieval). The second type of representations reflects information that cannot be deliberately retrieved, not edited by the individual, and is not contextualized in the autobiographical memory. This is called situationally accessible memory (SAM; e.g., direct retrieval).

Thus, the "two retrieval route" account seems to offer a good explanation for the co-occurrence of specific intrusions and overgeneral memories in depressed and traumatized samples. That is, higher distress including more frequent intrusions following trauma may result in truncated generative retrieval, for example via affect regulation or reduced executive capacity, and thus in reduced specificity on the standard AMT (e.g., Williams \& Broadbent, 1986). 
However, ironically, the intrusions that are a key source of the distress in the first place remain relatively unaffected by truncated generative autobiographical memory retrieval because they are habitually directly retrieved. In other words, affect regulation or reduced executive capacity in response to trauma-related intrusions and distress seem to influence generative retrieval rather than direct retrieval.

\section{Methodological remarks}

The research presented in the current dissertation gives rise to some methodological implications. Several of them relate to the separate studies and have already been reported in the previous Chapters. Here, a few more general themes are discussed.

\section{The use of analogue samples}

The first topic relates to the samples in which intrusions and overgeneral memory are assessed. Both intrusions and overgeneral memory appear mainly (sub)clinical phenomena (Brewin, 2007; Moore \& Zoellner, 2007; Williams et al., 2007). Accordingly, when the natural occurrence of intrusions (in relation to a particular experience) and overgeneral memories are assessed in a non-clinical sample, a relatively low number of intrusions (e.g., Berntsen, 2001) will be reported and a relatively high number of specific autobiographical memories (e.g., Merckelbach, Muris, \& Horselenberg, 1996). The results described in Chapter 2, indeed, show that non-clinical participants were relatively specific in their autobiographical memory retrieval. Furthermore, although the range of self-reported intrusions was considerable, the mean number of intrusions was relatively low. For these reasons, it may not be surprising that no association between the two phenomena was found. For example, floor and ceiling effects may have hampered the effect. However, the data did extend the previously found association of overgeneral memory and avoidance symptoms in (sub)clinical samples to a non-clinical sample.

Chapter 5 also used a student sample to investigate the (sub)clinical phenomenon of intrusions and overgeneral memory. Here, the aim was to explore the direction of the relationship between intrusions and reduced memory specificity more directly (e.g., which of the two phenomena precedes 
the other). Specifically, we were interested in the effect of experimentally induced intrusions about an aversive life event on subsequent autobiographical memory specificity. For such a research question, a sample in which both phenomena are relatively absent before starting the experiment may be desirable, because it is possible to elicit symptomatology (e.g., intrusions) and examine its effect on other phenomena (e.g., autobiographical memory specificity) while controlling for any other spurious factors.

Somewhat differently, the studies in Chapters 6 and 7 included community samples that were characterized by a (naturally occurring) specific negatively stressful situation or a history of a particular traumatic experience. Such samples can be useful to model traumatic experiences, because traumatic experiences are complicated to elicit or manipulate due to ethical constraints. Here, an evident relationship of self-reported intrusions and avoidance symptoms with reduced memory specificity was obtained in women with pregnancy complications (Chapter 7) and individuals reporting a history of CSA (Chapter 6). In both samples, the number of intrusions was, indeed, higher and fewer specific memories were recalled than in the student samples in Chapters 2 and 5 that reported symptoms with respect to aversive life events. Additionally, Chapter 6 showed that CSA participants with experience-related symptoms were even more overgeneral than those reporting fewer symptoms.

Taken together, subjects like restriction of range and floor or ceiling effects may complicate research when assessing correlations between trauma-related intrusions, avoidance symptoms and autobiographical memory specificity in analogue samples. For this reason (sub)clinical samples may be better to investigate the naturally occurring association between intrusions and overgeneral memory (see also Moore \& Zoellner, 2007). Still, when examining the underlying mechanisms, analogue samples may be preferred to carry out experimental manipulations (e.g., Chapter 5). This has to do with the fact that with such an approach, spurious factors can be better controlled for. Nevertheless, one should be aware that the manipulations employed elicit enough of the modeled symptomatology and that the tasks and measures of interest are sensitive enough to assess differences. 


\section{Manipulation of intrusions}

The central theme of the present thesis was to explore how the association between intrusions and reduced autobiographical memory specificity could be explained. To investigate the link between these phenomena, one approach is to induce intrusions that have the potential to influence autobiographical memory specificity. Intrusions consist of characteristics like involuntariness, trauma-relatedness, self-relevance, negative valence, and sensory richness that preferably should be modeled or approached while inducing them in the lab. To this end, in Chapters 3 and 4, we chose to explore whether retrieval-induced forgetting could account for the co-occurrence of intrusions and overgeneral memory (Wessel et al., 2002). Thus, intrusions were modeled via repeated retrieval of autobiographical memories (Chapter 3 ) and visual details of a scene (Chapter 4) in a retrieval practice paradigm and its effect on (autobiographical) memory specificity was investigated. Whereas interesting results have been obtained with this method, one may question how applicable this method is to model the clinical memory phenomenon of interest. For example, the involuntariness characteristic of intrusions was not taken into account.

In the literature, other methods to elicit intrusions are reported. Among these are aversive trauma films (e.g., Holmes, Brewin, \& Hennesy, 2004) and thought suppression (e.g., Barnier, Levin, \& Maher, 2004; Wegner, 1994). Regarding trauma films, after having seen such a film, participants may be asked to keep a diary for one week to report all intrusive thoughts that they have about the aversive film (e.g., Holmes et al., 2004; Stuart, Holmes, \& Brewin, 2006). Such a method has proven to be good to elicit involuntary intrusive recollections that include sensoric details (e.g., Holmes et al., 2004; Stuart et al., 2006). However, the material is not autobiographical or self-related. Furthermore, successful employment of the diary method highly depends on participants that really keep their diaries with them for a week and note every film-related intrusion that they experience during the day.

In case of the thought suppression paradigm, participants are asked to report intrusive thoughts related to the suppressed event during suppression and shortly thereafter (e.g., Wenzlaff \& Wegner, 2000). The diary method has also been applied in this setting to assess the more long-term of thought 
suppression on intrusive thoughts (Geraerts, Merckelbach, Jelicic, \& Smeets, 2006). An advantage of this method is that it can be applied to a wide range of material, including autobiographical memories. Then again, the success of the thought suppression manipulation depends 1 ) on a well-performed instruction to the participants, and 2) on motivated participants who indeed press a button every time when they experience an intrusion related to the suppressed event.

To our knowledge, so far, both methods have only been used to elicit intrusions, but not to investigate the effect of intrusions on subsequent other memory performance. The study presented in Chapter 5 was, therefore, to our knowledge the first that used thought suppression to elicit intrusions and examine the effect of suppression and intrusions on subsequent autobiographical memory retrieval. Although the effects were relatively small, the data tended to show that intrusions that were reported during the AMT were related to reduced autobiographical memory specificity.

To date, there is not yet an optimal method to induce intrusions and investigate the effect on subsequent (autobiographical) memory performance. In future, the use of thought suppression and aversive trauma films may be a fruitful avenue to pursue research in this domain. Furthermore, in Chapter 5 we only explored the relative direct effects of suppression and intrusions on autobiographical memory specificity. Possibly, long-term effects are larger and more meaningful in the light of ecological validation. Using the diary method to monitor intrusions during the week after the experimental manipulation and subsequently administer another AMT might allow us to examine whether autobiographical memory specificity is affected in the longer term.

\section{Assessment of overgeneral memory: The AMT}

The third methodological topic relates to the assessment of autobiographical memory specificity. So far, the cueing methodology - known as the Autobiographical Memory Test (AMT) in this field of research (Williams \& Broadbent, 1986) - has been primarily used to assess autobiographical memory specificity. In a way, there is a danger for circular reasoning: it seems that we measure overgeneral memory with the AMT and what we measure with the AMT, we term overgeneral memory. Although many studies report correlations between the AMT and a variety of constructs (see Williams et al., 2007), 
relatively little research has looked at the validity and reliability of the AMT as an assessment tool.

Only some studies have measured reduced memory specificity using other tasks than the AMT (Meesters, Merckelbach, Muris, \& Wessel, 2000; Orbach, Lamb, Sternberg, Williams, \& Dawud-Noursi, 2001). However, the AMT was not assessed in parallel in these studies. Therefore, it remains uncertain whether the same construct was measured. Furthermore, with the standard AMT, it is difficult to check whether participants are really recalling an autobiographical memory or whether they understand the task demands and just make up a report that meets the task criteria. Thus, questions arise about what the AMT exactly measures, and whether it is an isolated memory phenomenon or is a more general memory deficit.

Gibbs and Rude (2004) compared the AMT with a short autobiographical essay task and they found a positive relationship between the AMT and the autobiographical essay task. In a similar vein, Raes and his colleagues (2006) investigated whether autobiographical memory retrieval on an AMT would be related to reduced executive capacity (see also Dalgleish et al., 2007) and to source memory (see also Ramponi, Barnard, \& Nimmo-Smith, 2004). Their findings show that autobiographical memory specificity is positively related to measures of executive capacity (e.g., working memory) and to source memory. That is, participants with a more overgeneral retrieval style also had more difficulty with defining the correct source of a particular word that they had learned on a wordlist learning task. This finding indicates that autobiographical memory specificity measured with the AMT is not an isolated memory phenomenon. However, the AMT is not just interchangeable with, for example, any other memory task in explaining variance in clinically relevant depressive and posttraumatic stress symptoms (e.g., Wessel et al., 2002).

There are also differences between experiments in how the AMT is assessed (see van Vreeswijk \& de Wilde, 2004 for a meta analysis). For example, most studies visually and orally presented cue words on the AMT, but several studies used a written format. Similarly, most studies employed 60s as a time limit for participants to come up with a specific autobiographical memory. However, 30s has been used as a time limit in non-clinical participants and in some studies no 
time limit has been reported at all. Nevertheless, van Vreeswijk and de Wilde (2004) noted that these procedural differences do not greatly affect what the AMT measures.

Finally, the data of Chapter 6 deserve some special attention here. In this Chapter, we demonstrated in a sample of individuals with a history of CSA that, reduced memory specificity depends on the type of cues that are presented (see also Williams, Ellis, Tyers, Macleod, \& Rose, 1996; Williams, Healy, \& Ellis, 1999). Both CSA and control participants retrieved more specific autobiographical memories in response to highly imageable and concrete word cues as compared to the more low imageable abstract word cues on the standard AMT (Williams \& Broadbent, 1998). Important to note in this respect is that the results of Chapter 6 were not due to a low discriminating power of AMT consisting of the highly imageable and concrete word cues: participants did not retrieve more specific memories on this AMT just because the task was easier. Thus, in future, care should be taken with respect to which cues are used in an AMT to elicit specific autobiographical memories because it is plausible that different retrieval processes may be measured depending on the type of cue word used.

Altogether, whatever the AMT exactly measures, it appears a cognitive memory and capacity task that does have clinical value (see Moore \& Zoellner, 2007; Williams et al., 2007). Its clinical relevance becomes apparent in studies reporting, for example, that AMT performance is a predictor of poor treatment outcome (e.g., Brittlebank, Scott, Williams, \& Ferrier, 1993). Even though overgeneral memory is not an isolated memory phenomenon (e.g., Dalgleish et al., 2007; Raes et al., 2006; Ramponi et al., 2004) and more studies are recommended on the validity and reliability of the AMT, overgeneral memory is consistently found in clinical and subclinical populations (see Williams et al., 2007). Apart from an important role of reduced executive capacity and functional avoidance, self-relatedness and the goal directing function of specific autobiographical memories (e.g., Conway, 2005; Conway et al., 2004) may help to explain why exactly overgeneral AMT performance has clinical ramifications. 


\section{Directions for future research}

In addition to the above-mentioned suggestions for future research, this section addresses some more ideas for further research on overgeneral memory and its relation to trauma-related symptoms. In their thorough review of overgeneral memory and trauma, Moore and Zoellner (2007) indicate that, so far, most of the research on this relationship has focused on the role of exposure to traumatic events (e.g., Williams, 1996; Williams et al., 1999). Consequently, relatively little attention has been paid to alternative theories and mechanisms (e.g., Berntsen \& Rubin, 2006; Philippot, Schaefer, \& Herbette, 2003) that may be responsible for overgeneral memory (Moore \& Zoellner, 2007). Many studies, including the ones in the present dissertation, imply that mere exposure to traumatic events alone is not enough to develop an overgeneral memory retrieval style (see Moore \& Zoellner, 2007; Williams et al., 2007). Therefore, more research derived from alternative theories should be conducted. Furthermore, more studies are recommended that thoroughly control for possibly relevant confounding variables such as a history of trauma and depression, as well as studies that use standardized diagnostic interviews to assess severity and diagnostic status for both PTSD and depression (Moore \& Zoellner, 2007; see also Williams et al., 2007).

\section{Two retrieval-route account}

Concurring with the two retrieval-route account to explain the co-occurrence of specific intrusions (via a direct retrieval process) and overgeneral memory (via a generative retrieval process; e.g., Conway et al., 2004; Williams et al., 2007), additional studies manipulating cue type and content are recommended. If in traumatized and depressed individuals, the direct retrieval process is still intact, but the generative retrieval route is disturbed, then these individuals should report more specific memories to perceptual cues such as visual images, objects, and odors than to word cues. Some experiments indeed report results in this direction (e.g., Chu \& Downes, 2002; Goddard, Pring, \& Felmingham, 2005).

Goddard et al. (2005) conducted a study in which they asked healthy participants to retrieve specific memories to word cues, visual image cues, and odor cues. They found that participants retrieved significantly fewer specific 
memories to odor cues in comparison to word and visual cues. Furthermore, Chu and Downes (2002) reported that participants retrieved more detailed autobiographical memories in response to the combination of a verbal and odor cue than to a verbal and visual cue. In another study, Schönfeld and Ehlers (2006) reported that visual cues elicited more specific memories than word cues in individuals with PTSD. It would be interesting to examine whether relative to healthy individuals, individuals with depressive and posttraumatic symptomatology and with a formal diagnosis of PTSD would retrieve more specific memories in relation to odor cues and other stimuli with a strong perceptual loading than to word cues on an AMT.

Another line of research that might be interesting is to combine the assessment of the standard and concrete AMTs with other tasks that measure the same constructs. For example, if the standard AMT measures generative retrieval that uses limited cognitive capacity, participants should perform in a similar way on other (memory) tasks that measure conceptual/verbal or generative processing. In a similar way, if the concrete AMT measures direct retrieval that hardly needs cognitive capacity, performance on this task should correlate with other tasks measuring perceptual/sensoric or direct processing. The two retrieval-route account would gain in strength if this would be true for both healthy participants and for participants displaying overgeneral and intrusive memories.

\section{Disintegration or enhanced integration of trauma memories}

As outlined in the introduction, Ehlers and Clark (2000) argue that a traumatic experience leads to schema violation and that this may lead to a lack of cognitive integration between parts of the traumatic memory that are deliberately retrieved and memory parts that are only accessible via concrete (direct) retrieval cues. Consequently, PTSD may develop. Williams et al.'s (2007) account on overgeneral memory concurs with this view (see also Dalgleish, 2004). There is, however, evidence against the view that trauma memories are not very well integrated (e.g., Merckelbach, Dekkers, Wessel, \& Roefs, 2003a,b; Geraerts et al., 2007). For example, Geraerts et al. (2007) demonstrated in a sample of war veterans with and without PTSD that trauma memories were not qualitatively different from neutral memories regarding 
stability over time and sensory qualities. Furthermore, PTSD symptom severity was not associated with dissociative experiences.

These findings may be better accommodated by Berntsen and Rubin (2007), who believe that PTSD develops due to the traumatic memory being extremely well integrated into autobiographical memory. It may even become a dysfunctional reference point into ones life to which many other autobiographical memories have become linked (e.g., vivid landmark, Berntsen, Willert, \& Rubin, 2003). Thus, rather than arguing that schema violation in the context of trauma leads to poor or fragmented memories (e.g., Ehlers \& Clark, 2000), Berntsen and Rubin (2007) argue that there is ample evidence that schema violation enhances memory (e.g., Brown \& Kulik, 1977; Rubin \& Kozin, 1984). Furthermore, they believe that traumatic memories do not qualitatively differ from non-traumatic memories (e.g., Berntsen, 2001; see also Brewin, 2007; Porter \& Birt, 2001; Shobe \& Kihlstrom, 1997 ).

Berntsen and Rubin (2006) developed a questionnaire, the Centrality of Events Scale (CES), that measures the extent to which a traumatic memory forms a reference point into ones life. They found that the CES score was positively related to the severity of posttraumatic stress symptoms, even after controlling for measures of anxiety, depression, dissociation, and self-consciousness (Berntsen \& Rubin, 2007). A question that may arise is whether (hyper)centrality of trauma memories may also lead to an overgeneral autobiographical memory for events that are not related to the traumatic experience.

A first step would be to examine whether overgeneral memory performance on the AMT (Williams \& Broadbent, 1986) would positively correlate with the CES. Furthermore, this account would suggest that traumatized individuals would be able to retrieve specific memories about the trauma, but not about other (i.e., less central, trauma-unrelated) themes (see also Golden et al., in press). Thus, asking participants to retrieve specific memories in relation to their trauma and other themes in their lives should lead to different specificity scores. Somewhat different to Golden et al. (in press), a study by McNally, Litz, Prassas, Shin, \& Weathers (1994) also seems to point in that direction. In this study, Vietnam War veterans with and without PTSD were asked to retrieve memories to cue words from the Vietnam periods and other periods. The authors found that 
regalia wearing veterans with PTSD retrieved more specific autobiographical memories from the Vietnam period than those who did not wear regalia. Thus, those individuals for whom the trauma still hold a prominent place in their lives retrieved more specific trauma-related memories than those individuals for whom this was not the case.

Finally, it might be interesting to investigate what happens with autobiographical memory specificity when healthy participants are first asked to make a negative or positive memory a reference point in their minds. If Berntsen and Rubin's (2006) analysis is correct, participants who engaged in such a manipulation should subsequently become less specific when asked to retrieve non-traumarelated autobiographical memories. This should be true when both the positive and negative experiences that have become a reference point. Taken together, in the light of examining alternative theories besides falsifying already existing theories about overgeneral memory (e.g., Moore \& Zoellner, 2007; Williams et al., 2007), the Berntsen and Rubin account may be an interesting direction to advance future research in the field of autobiographical memory retrieval and psychopathology.

\section{Practical implications}

\section{Clinical practice}

Prior research has demonstrated that overgeneral memory is accompanied by poor problem solving skills and poor articulation of future goals (see Williams et al., 2007). Furthermore, reduced autobiographical memory specificity is a vulnerability factor to develop depressive symptoms and it predicts poor outcome after therapy (see Williams et al., 2007). Whereas Harvey, Bryant, and Dang (1998) found that patients with Acute Stress Disorder (ASD) displaying reduced memory specificity were having more PTSD symptoms six moths later, the data of Chapter 7 demonstrate for the first time that pre-trauma reduced autobiographical memory specificity also predicts the development of posttraumatic stress symptoms in a community sample without a formal diagnosis. A question that arises from these and the prior prospective data is whether overgeneral memory as a predictor at group level has also predictive value at the individual level. Does the AMT have clinical importance to predict whether an individual is at risk to develop posttraumatic and/or depressive 
symptoms in the near future? In future, the assessment of overgeneral memory retrieval may have diagnostic relevance to assess the risk to develop later posttraumatic and depressive symptoms.

Another clinical application related to autobiographical memory specificity is to train depressed and traumatized individuals on the retrieval of specific memories. Therapies like mindfulness based cognitive therapy (Williams, Teasdale, Segal, \& Soulsby, 2000) and Pennebaker's (1995) diary/bibliography therapy have taken the first steps in this direction. Also techniques based on rumination have shown that participants can learn to retrieve more specific autobiographical memories (Watkins, Teasdale, \& Williams, 2000). Besides the research on rumination as a source to develop training for specific memory retrieval, the data in Chapter 6 show that effortful retrieval is impaired whereas direct retrieval remains intact. This finding may inspire other training techniques. Training the generative search process, but also, for example, trying to enhance executive capacity (e.g., Dalgleish et al., 2007; Hertel, 2000), might help these people to become better able to retrieve specific autobiographical memories. This, in turn, may improve their problem solving skills and goal directed behavior.

\section{Forensic setting}

In a forensic setting where traumatized people may be repeatedly interrogated or interviewed about past (traumatic) events the validity and reliability of memory is relevant. People having an overgeneral memory may be more vulnerable to making memory errors, because they are less able to retrieve specific details of memories. The point here is, of course, that vague, overgeneral memories of a defendant may elicit stronger interrogative pressure by the interrogators. Consequently, individuals with an overgeneral memory may be more susceptible to different kinds of memory errors.

Prior research has already demonstrated that memory distortions like source monitoring errors are associated with overgeneral responses on an AMT (Raes et al., 2006; Ramponi et al., 2004). Individuals with an overgeneral memory might also be more susceptible to suggestion (e.g., Gudjonsson \& Clare, 1995) and to false memories (e.g., misinformation effect, Loftus, 2005). So far, no studies have been done on suggestibility and autobiographical memory 
specificity. Furthermore, to our knowledge, only one study has related performance on the AMT to misinformation tasks in a student sample (Buijsrogge, 2007). However, no relationship was found between reduced autobiographical memory specificity and a higher susceptibility to the misinformation effect.

Therefore, more empirical studies are recommended that combine the AMT with measures of suggestibility (e.g., Gudjonsson, 1989), misinformation paradigms (e.g., Loftus, 2005), or with paradigms of false memories (e.g., Deese-Roediger McDermott paradigm, Roediger \& McDermott, 1995). Such experiments may give more insight in how overgeneral memory and susceptibility to memory distortions are related to each other. Nevertheless, we believe that extra care should be taken during the interrogation of individuals with a history of trauma or depression, because their overgeneral memory retrieval style may be easily mistaken for an attempt to hide information. Furthermore, prior research on the credibility of accounts of asylum seekers has shown that refugees with higher levels of posttraumatic stress are more likely to be inconsistent in their account when there is more time in between the interviews. What is more, asylum seekers were more likely to be inconsistent in details that they rated as peripheral than those they rated as central (Herlihy, Scragg, \& Turner, 2002).

The findings reported in Chapters 3 and 4 may also be relevant for the forensic setting. In Chapter 3 , repeated retrieval of a subset of autobiographical memories causes the recall of fewer unrehearsed memories. In a different way, the data in Chapter 4 show that selective retrieval of either central or peripheral details from a negatively valenced visual scene causes more recall errors in unrehearsed related information. This is relevant for interrogation situations in which some information may be repeatedly focused on during a police interview. This focus may subsequently impair memory for other details that later turn out to be important, a phenomenon that was demonstrated in a study by Wright, Loftus, and Hall (2001). As a consequence, eyewitnesses or suspects may have superior retrieval for the material that the police focused on during interrogation. However, other details may have been forgotten in the meanwhile, become distorted, or new details are erroneously added. Policemen and other interrogators should be aware of these processes when they are assessing the reliability and accuracy of eyewitness reports or suspects' statements. 


\section{Closing remarks}

For a long time, research on overgeneral autobiographical memory has mainly focused on its relationship with prior trauma because of the dominating idea that overgeneral autobiographical memory retrieval is a way to avoid negative affect and distress that accompanies retrieval of trauma memories (Williams, 1996; see also Moore \& Zoellner, 2007). To this end, the functional avoidance account (Williams, 1996; Williams et al., 1999) has been the leading theory to explain overgeneral memory retrieval in depressed and traumatized individuals. However, it has become apparent that the presence of prior trauma alone is, in most instances, not enough to account for overgeneral memory retrieval and that the role of trauma-related symptoms is more crucial in this respect (e.g., Moore \& Zoellner, 2007; Williams et al., 2007).

Research from the last 10 years has, indeed, demonstrated the importance of trauma-related symptomatology for overgeneral autobiographical memory retrieval. The data of the present dissertation clearly add to this finding. Additional research has shown that other processes such as rumination (e.g., Watkins \& Teasdale, 2001; 2004) and reduced executive capacity (Dalgleish et al., 2007) can account for overgeneral memory retrieval (see also Williams et al., 2007). Nonetheless, so far, empirical evidence trying to falsify the functional avoidance account, which is still a major theory in this field of research, is limited, the same as is research testing alternative explanations (e.g., Moore \& Zoellner, 2007). The different studies presented in the current dissertation were such an attempt.

The findings and ideas presented in this thesis may inspire future research to design experiments that try to discriminate between different accounts of overgeneral autobiographical memory retrieval. For example, Dalgleish, Rolfe, Golden, Dunn, and Barnard (in press) compared the affect regulation view with the reduced executive capacity account in a sample of individuals with PTSD. They assessed a reversed AMT (i.e., asking participants to retrieve overgeneral instead of specific memories in response to cue words) and associated outcome on the AMT with PTSD symptom severity. Whereas affect regulation would predict a negative association between symptom severity and memory specificity, the reduced executive capacity account would suggest the opposite 
(i.e., symptom severity would be positively related to 'errors' on the AMT). The results supported the affect regulation account. In a similar vein, the functional avoidance account suggests an opposite mechanism why intrusions and overgeneral memory co-occur than does the account of Berntsen and Rubin (2006). Such studies may further help to elucidate the unique role of specific autobiographical memory retrieval in relation to vulnerability and maintenance of emotional disorders. At the end of the day, our autobiographical memory is central to who we are and what we do. Thinking back of that day in Venice... 

Samenvatting 
Stelt u zich het volgende voor: Je denkt terug aan die ene dag in Venetië een aantal jaren geleden. Je was 's ochtends vroeg op het San Marco plein aan het wandelen. Het plein was helemaal bedekt met duiven en verder was er niemand, behalve jij. Je ruikt de geur weer van de verse Italiaanse espresso en je voelt je wederom helemaal gelukkig, net zoals die ochtend. Het was een mooi moment.

Dit is een voorbeeld van een autobiografische herinnering. Het autobiografische geheugen is een onderdeel van het episodische geheugen (Tulving, 2002). Met behulp van het episodische geheugen kunnen wij gebeurtenissen uit het verleden overdenken. We kunnen hierdoor ook mentaal door de tijd reizen en een gebeurtenis uit het verleden herbeleven. Het ophalen van een autobiografische herinnering is daarom meer dan alleen een objectieve beschrijving van feiten (zoals een semantische herinnering, 'Wanneer ben je jarig?' en 'Waar woon je?'). Wanneer je een autobiografische herinnering ophaalt, dan weet je dat het gaat om een persoonlijke gebeurtenis uit je verleden die onder meer bestaat uit je eigen interpretatie, emoties, gedachten, maar ook uit beelden, geluiden of geuren (perceptuele of sensorische details). Alles samengenomen, episodische en zelfrelevante informatie zijn belangrijke onderdelen van een autobiografische herinnering.

Doorgaans zijn er twee manieren waarop we herinneringen ophalen. De eerste is dat je bewust en actief op zoek gaat naar een bepaalde herinnering, bijvoorbeeld je mooiste vakantie herinnering. Deze manier van ophalen heet generative retrieval. Maar soms komt er ineens een herinnering in je op zonder dat je moeite hebt gedaan om deze te herinneren. Dit is dan meestal naar aanleiding van een ophaalaanwijzing in de omgeving, bijvoorbeeld de geur van koffie, een persoon of een kleur. Deze vorm van ophalen wordt direct retrieval genoemd.

$\mathrm{Er}$ is veel onderzoek gedaan naar autobiografisch geheugen, bijvoorbeeld over het opslaan, ophalen en vergeten van persoonlijke gebeurtenissen (bv. Tulving, 2000). Maar ook over de functie van autobiografische herinneringen (bv. Pillemer, 1998; Rubin, 1996) en de relatie met (zelf)ontwikkeling (Conway et al., 2004; Nelson \& Fivush, 2004). Het onderzoek dat is beschreven in dit proefschrift is ingebed in het Self Memory System over autobiografisch 
geheugen (Conway et al., 2004). Dit model verklaart autobiografisch geheugen in relatie tot het zelfconcept en de korte en lange termijn doelen van mensen. Het autobiografische geheugen is mede daarom een belangrijk aspect van het dagelijks functioneren van mensen (Conway et al., 2004). Het helpt ons bijvoorbeeld om nieuwe ervaringen betekenis te geven, om plannen te maken voor de toekomst, en het draagt bij tot onze identiteit en zelfbewustzijn (Conway et al., 2004).

Uit het voorgaande zal het voorstelbaar zijn dat een verstoord autobiografisch geheugen een belangrijke rol kan spelen binnen de psychopathologie. Onderzoek heeft inderdaad aangetoond dat vooral emotionele stoornissen, met name depressie en posttraumatische stress, worden gekenmerkt door systematisch vervormde ophaalpatronen van autobiografische herinneringen (bv. Conway et al., 2004). Zo rapporteren mensen met een depressie en/of een traumatisch verleden veelvuldig specifieke intrusieve en herbelevingen van de traumatische gebeurtenis. Kortweg ook wel intrusies genoemd. Intrusies zijn onvrijwillig en komen voortdurend op in het bewustzijn. Ze geven een gevoel alsof de ingrijpende ervaring weer gebeurt en zijn daarom meestal zeer verontrustend.

Tegelijkertijd zijn dezelfde mensen geneigd om overwegend overalgemene autobiografische herinneringen op te halen, wanneer ze zich doelbewust een persoonlijke gebeurtenis willen herinneren. In tegenstelling tot de specifieke autobiografische herinnering die hierboven cursief staat beschreven, is een overalgemene herinnering meestal een categorie aan persoonlijke gebeurtenissen, zoals 'Telkens als ik op vakantie ga', of een herinnering aan een periode, 'Die vakantie in Italië in de zomer van 2000'. Mensen die geneigd zijn om voornamelijk dit soort overalgemene autobiografische herinneringen op te halen, hebben meer moeite met het oplossen van sociale problemen, blijken gevoeliger om depressieve en posttraumatische stress klachten te ontwikkelen en knappen minder snel op uit therapie (bv. Williams et al., 2007). De samenhang tussen een overalgemeen autobiografisch geheugen en intrusieve herinneringen is inderdaad gevonden in diverse groepen, bijvoorbeeld slachtoffers van brandwonden (Stokes et al., 2004), aanranding (Schönfeld et al., 2007), oorlogstrauma (Wessel et al., 2002), of mensen met depressie (bv. Hermans et al., 2004). 
In dit ophaalpatroon van autobiografische herinneringen lijkt echter een tegenstelling aanwezig. Enerzijds hebben mensen met depressieve en posttraumatische stress klachten last van zeer specifieke traumagerelateerde intrusies die steeds in het bewustzijn opkomen. Maar anderzijds hebben ze moeite om doelbewust specifieke autobiografische herinneringen op te halen. Hoe kan deze ogenschijnlijke tegenstelling worden verklaard? Deze vraag staat centraal in dit proefschrift. In de empirische hoofdstukken wordt ingegaan op een aantal mogelijke verklaringen. Hieronder volgt een beknopt overzicht.

Tot nu toe is in het onderzoek naar overalgemeen geheugen veel aandacht besteed aan de relatie met traumatische ervaringen. Een dominante theorie in dit veld is de functional avoidance hypothese (Williams et al., 2007) die aangeeft dat overalgemene autobiografische herinneringen ontstaan als een soort defensiemechanisme tegen negatieve gevoelens. Het ophalen van specifieke herinneringen haalt te veel negatieve (traumatische) onderwerpen naar boven. Daarom wordt het zoekproces naar specifieke herinneringen iedere keer vervroegd afgebroken en worden alleen maar overalgemene herinneringen gerapporteerd. Vanwege de emotionele winst die dit oplevert, houdt dit proces zichzelf in stand. Mogelijk is deze passieve vermijding van negatieve onderwerpen niet altijd succesvol, waardoor intrusies toch de kans krijgen om in het bewustzijn te komen. Je kunt dit vergelijken met bepaalde gedachten die je actief probeert te vermijden. Dit heeft vaak de averechtse uitwerking dat je die gedachte juist voortdurend in je hoofd hebt. Het ophalen van overalgemene herinneringen is in deze situatie dus meer een disfunctioneel defensie mechanisme.

De link tussen intrusies en overalgemeen geheugen zou ook kunnen worden verklaard door een verminderde capaciteit (reduced cognitive capacity) om cognitieve taken uit te voeren (Dalgleish et al., 2007; Williams et al., 2007). Het ophalen van herinneringen is een cognitieve taak en kost energie. Juist die energie is beperkt bij mensen met depressieve en posttraumatische stress klachten. Door de verminderde cognitieve capaciteit wordt het zoekproces naar specifieke autobiografische herinneringen vervroegd afgebroken omdat er geen energie meer is om verder te zoeken. Het gevolg is dat overalgemene autobiografische herinneringen worden opgehaald. Tegelijkertijd zorgt een 
verminderde cognitieve capaciteit er ook voor dat ongewenste of irrelevante informatie, zoals intrusieve herinneringen, minder goed kan worden genegeerd.

Een geheel andere mogelijke verklaring voor de samenhang tussen overalgemeen geheugen en intrusieve herinneringen komt uit de retrievalinduced forgetting - vergeten door te herinneren - literatuur (Anderson, 2003). Meerdere onderzoeken hebben aangetoond dat het herinneren van bepaalde informatie het vergeten van andere informatie tot gevolg heeft. Het idee hier is dat voortdurende intrusieve herinneringen het ophalen van andere autobiografische herinneringen bemoeilijkt, wat uiteindelijk zou kunnen resulteren in een overalgemeen autobiografisch geheugen (Wessel et al., 2002). In deze verklaring gebruiken intrusies niet alleen de beperkte cognitieve capaciteit (zoals in de verklaring hierboven), maar dragen ze ook bij tot een verminderde beschikbaarheid van andere autobiografische herinneringen. Dit betekent dat deze herinneringen steeds moeilijker op te halen zijn, onafhankelijk van welke ophaalaanwijzing wordt gebruikt.

Een laatste hypothese om de samenhang tussen intrusies en overalgemeen geheugen te verklaren is dat ze beide afhankelijk zijn van verschillende ophaalprocessen. Intrusies worden spontaan opgehaald via een direct retrieval proces en het doelbewust ophalen van autobiografische herinneringen verloopt via een generative retrieval proces. Het idee is dat overalgemene herinneringen ontstaan omdat het generative retrieval proces is verstoord, terwijl het direct retrieval proces nog gewoon intact is. Daardoor blijven intrusies de vrije hand houden om voortdurend in het bewustzijn op te komen.

\section{Overzicht van empirische bevindingen}

Eerder onderzoek bij mensen met een depressie en/of een traumatisch verleden heeft aangetoond dat er een relatie is tussen overalgemeen geheugen enerzijds en intrusies en vermijdingssymptomen anderzijds (bv. Kuyken \& Brewin, 1995; Schönfeld \& Ehlers, 2006). Ander onderzoek suggereert dat intrusies (bv., Berntsen, 2001), de neiging om ongewenste herinneringen te vermijden (Wenzlaff \& Wegner, 1996), en het relatieve onvermogen om specifieke herinneringen op te halen (Merckelbach, Muris, \& Horselenberg, 1996) ook in non-klinische groepen voorkomt. De studie in Hoofdstuk 2 onderzocht daarom of de samenhang van overalgemeen autobiografisch 
geheugen met intrusies en vermijdingssymptomen gerepliceerd kon worden in een groep van studenten. We vonden geen significante correlatie tussen overalgemeen geheugen en zelfgerapporteerde intrusies. Maar de resultaten lieten wel een positieve associatie zien tussen vermijdingssymptomen en overalgemene herinneringen die werden gerapporteerd naar aanleiding van negatieve steekwoorden op de autobiografische geheugentest (Williams \& Broadbent, 1986). Deze associatie bleef significant nadat we hadden gecontroleerd voor depressieve symptomen en de recentheid van de ingrijpende gebeurtenissen die door de proefpersonen werden beschreven. Deze bevindingen repliceren dus de eerder gevonden individuele verschillen op de autobiografische geheugentest in een groep van studenten.

De twee studies die worden beschreven in Hoofdstuk 3 onderzochten of het retrieval-induced forgetting (vergeten door te herinneren) effect ook bij autobiografische herinneringen voorkomt. Meestal wordt overalgemeen geheugen gemeten met de autobiografische geheugentest die doorgaans bestaat uit een brede reeks van ophaalaanwijzingen (hier: steekwoorden). In tegenstelling tot de autobiografische geheugentest, gebruikt het retrieval practice paradigma (ophaal-oefen paradigma) juist een kleine categorie aan ophaalaanwijzingen (bv. Anderson, Bjork, \& Bjork, 1994; Barnier, Hung, \& Conway, 2004). In de eerste studie werd het retrieval practice paradigma toegepast op meer brede categorieën van positieve en negatieve autobiografische herinneringen. De resultaten lieten inderdaad het retrievalinduced forgetting effect zien, net als de eerdere resultaten van Barnier et al. (2004). Maar omdat het effect vrij klein was hebben we een tweede studie gedaan met alleen negatieve autobiografische herinneringen. De data lieten dit keer een groter retrieval-induced forgetting effect zien. De bevindingen toonden verder aan dat het effect hoogstwaarschijnlijk door een non-inhibitoir mechanisme is veroorzaakt omdat sommige van de geoefende herinneringen in de verkeerde categorie waren gerapporteerd (in plaats van helemaal niet gerapporteerd). Dit suggereert dat de herinneringen niet minder beschikbaar zijn geworden (bv. Anderson, 2003), maar in een verkeerde context zijn gerapporteerd.

De drie studies die worden beschreven in Hoofdstuk 4 bestudeerden of retrieval-induced forgetting ook bij emotioneel negatieve foto's voorkomt. Deze 
studies waren geïnspireerd door het aandachtsvernauwingseffect (bv. Reisberg \& Heuer, 2004) en het feit dat intrusies perceptuele en sensorische details bevatten (bv. Conway et al., 2004). We bestudeerden hier niet het effect van herhaald ophalen van complete herinneringen op het ophalen van andere complete herinneringen (zoals in Hoofdstuk 3). Bij deze studies waren we juist geïnteresseerd in het effect van het ophalen van bepaalde details van een herinnering op het ophalen van andere details van diezelfde herinnering. Alledrie de studies toonden echter geen bewijs voor de hypothese dat herhaald herinneren van centrale of perifere details van emotionele foto's het herinneren van ongeoefende perifere en centrale details van diezelfde foto zou verslechteren. Proefpersonen die de centrale details (studies 1en 3) herhaald oefenden, maakten wel meer commissie fouten in de ongeoefende details tijdens de vrije ophaal fase. Dit betekent dat mensen die centrale details herhaald herinnerden, ook meer onjuiste details gingen noemen die ze niet hadden waargenomen. Zodoende toonden alledrie de studies geen bewijs voor een retrieval-induced forgetting verklaring in de context van het herinneren van negatieve emotionele foto's.

Hoofdstuk 5 beschrijft een studie waarin in een groep van niet-klinische studenten werd onderzocht of actieve gedachteonderdrukking (bv. Ehlers \& Clark, 2000; Wenzlaff \& Wegner, 2000) van iemands meest ingrijpende gebeurtenis meer intrusies over die gebeurtenis zou uitlokken, maar tegelijkertijd ook minder specifieke herinneringen op de autobiografische geheugentest. De negatieve conditie werd vergeleken met een conditie waarin een herinnering van een neutrale gebeurtenis werd onderdrukt. De resultaten toonden dat personen die meer intrusies over de gebeurtenis in kwestie rapporteerden ook minder specifieke herinneringen ophaalden. Dit effect was onafhankelijk van de instructie om de herinnering te onderdrukken en onafhankelijk van de valentie van gebeurtenis (negatief of neutraal). Verder was er een trend in de data dat die personen die de herinnering aan hun meest ingrijpende gebeurtenis onderdrukten, ook een minder negatieve stemming hadden dan personen die hun meest ingrijpende gebeurtenis niet onderdrukten (bv. Williams, Stiles, \& Shapiro, 1999).

Hoofdstuk 6 beschrijft een experiment waarin werd onderzocht of overalgemeen geheugen en intrusies afhankelijk zijn van twee verschillende ophaal processen 
(bv. Conway et al., 2004; Williams et al., 2007). De hypothese was dat het herinneren van intrusies gaat via directe associaties tussen ophaalaanwijzingen en specifieke herinneringen (direct retrieval), terwijl herinneringen die op de autobiografische geheugentest worden opgehaald juist het resultaat zijn van een iteratieve ophaalcyclus in een hiërarchisch systeem (generative retrieval). Om deze hypothese te onderzoeken, gebruikten we twee verschillende versies van de autobiografische geheugentest in een groep van mensen met en zonder een verleden van seksueel misbruik. De data ondersteunden onze hypothese dat seksueel misbruikte mensen meer overalgemene herinneringen zouden ophalen op de standaard autobiografische geheugentest (abstracte steekwoorden/ophaalaanwijzingen), maar dat dit verschil verdwenen zou zijn op de autobiografische geheugentest met directe (concrete) ophaalaanwijzingen. Dit effect was sterker voor seksueel misbruikte mensen met posttraumatische stress symptomen. Ook bleef het effect significant wanneer mensen met een depressieve stoornis (in het verleden) uit de analyses werden verwijderd. En wanneer alleen seksueel misbruikte mensen die extern bewijs voor hun misbruik hadden, werden geïncludeerd.

Hoofdstuk 7 beschrijft een prospectieve studie waarin werd onderzocht of overalgemeen autobiografisch geheugen een voorspeller is voor het ontwikkelen van posttraumatisch stress en depressieve symptomen na het beleven van een ingrijpende levensgebeurtenis (bv. Harvey, Bryant, \& Dang, 1998; Mackinger, Loschin, \& Leibetseder, 2000; van Minnen, Wessel, Smeenk, \& Verhaak, 2005). Om dit te onderzoeken werden vrouwen met zwangerschapcomplicaties twee keer getest: 1) direct na hun ziekenhuisopname en 2) zes weken na de bevalling. De data lieten zien dat vrouwen die op de autobiografische geheugentest minder specifieke herinneringen met een zwangerschapsgerelateerde inhoud (naar aanleiding van positieve steekwoorden) ophaalden, ook meer posttraumatische stress en depressieve symptomen rapporteerden zes weken na de bevalling. Dit effect bleef significant nadat de relevante variabelen werden gecovarieerd die zowel gerelateerd waren aan baseline autobiografische geheugen en follow-up posttraumatische stress en depressieve symptomen. 


\section{Conclusies}

De conclusies van het proefschrift worden beschreven en bediscussieerd in Hoofdstuk 8. Daarbij wordt aandacht besteed aan theoretische en methodologische aspecten, vervolgstappen voor nieuw onderzoek, en implicaties voor de klinische en forensische praktijk. De belangrijkste conclusies kunnen als volgt worden samengevat. De bevindingen uit de Hoofdstukken 3 en 4 geven geen ondersteuning voor een retrieval-induced forgetting verklaring voor de samenhang tussen intrusieve en overalgemene autobiografische herinneringen. Hoewel we in de studies in Hoofdstuk 3 vonden dat het ophalen van bepaalde autobiografische herinneringen het vergeten van andere herinneringen tot gevolg had, geven deze data samen met die uit Hoofdstuk 4 meer aanleiding tot andere onderzoekspaden.

De functional avoidance hypothese, daarentegen, kan de samenhang tussen intrusies en overalgemeen geheugen vrij aardig verklaren. Dit blijkt uit de data van de Hoofdstukken 2, 5, en 6 . Maar de bevindingen van Hoofdstuk 5 en 6 tonen tegelijkertijd dat deze verklaring onvolkomen en onbevredigend is. Met name Hoofdstuk 6 laat zien dat getraumatiseerde mensen, zowel onvrijwillig als doelbewust, nog steeds verontrustende herinneringen aan hun trauma ophalen. Deze resultaten kunnen wel verklaard worden door two retrieval routes hypothese, waarbij overalgemeen autobiografisch geheugen ontstaat door verstoorde generative retrieval, terwijl intrusies worden opgehaald via een direct retrieval proces dat nog prima intact is. Tot slot blijkt uit hoofdstuk 7 dat vrouwen die minder specifieke positieve zwangerschapsherinneringen kunnen ophalen meer kans hebben om depressieve en posttraumatische stress klachten te ontwikkelen na de bevalling. 



\section{References}

American Psychiatric Association. (1994). Diagnostic and Statistical Manual of Mental Disorders (4th ed.). Washington D.C.: American Psychiatric Association.

Amir, N., Coles, M. E., Brigidi, B., \& Foa, E. B. (2001). The effect of practice on recall of emotional information in individuals with generalized social phobia. Journal of Abnormal Psychology, 110, 76-82.

Anderson, M. C. (2003). Rethinking interference theory: Executive control and the mechanisms of forgetting. Journal of Memory and Language, 49, 415-445.

Anderson, M. C., Bjork, R. A., \& Bjork, E. L. (1994). Remembering can cause forgetting: Retrieval dynamics in long-term memory. Journal of Experimental Psychology: Learning, Memory, and Cognition, 20, 1063-1087.

Anderson, M. C., \& McCulloch, K. C. (1999). Integration as a general boundary condition on retrieval- induced forgetting. Journal of Experimental Psychology: Learning, Memory, and Cognition, 25, 608-629.

Anderson, M. C., \& Spellman, B. A. (1995). On the status of inhibitory mechanisms in cognition: Memory retrieval as a model case. Psychological Review, 102, 68-100.

Barnhofer, T., de Jong-Meyer, R., Kleinpass, A., \& Nikesch, S. (2002). Specificity of autobiographical memories in depression: An analysis of retrieval processes in a thinkaloud task. British Journal of Clinical Psychology, 41, 411-416.

Barnier, A. J., Hung, L., \& Conway, M. A. (2004). Retrieval-induced forgetting of emotional and unemotional autobiographical memories. Cognition and Emotion, 18, 457-477.

Barnier, A. J., Levin, K., \& Maher, A. (2004). Suppressing thoughts of past events: Are repressive copers good suppressors? Cognition and Emotion, 18, 513-531.

Bäuml, K. (1998). Strong items get suppressed, weak items do not: The role of item strength in output interference. Psychonomic Bulletin and Review, 5, 459-463.

Bäuml, K., \& Kuhbandner, C. (2004). Retrieval-induced forgetting and part-list cuing in associatively structured lists. Memory and Cognition, 31, 1188-1197.

Beck, A. T., Rush, A. J., Shaw, B. F., \& Emery, G. (1979). Cognitive therapy of depression. New York: Wiley.

Beck, A. T., Steer, R. A., \& Brown, G. K. (1996). Manual for the Beck Depression Inventory, 2nd ed. San Antonio: TX: The Psychological Corporation.

Beck, A. T., Ward, C. H., Mendelson, M., Mock, J., \& Erbaugh, J. (1961). An inventory for measuring depression. Archives of General Psychiatry, 4, 53-63.

Berntsen, D. (2001). Involuntary memories of emotional events: Do memories of traumas and extremely happy events differ? Applied Cognitive Psychology, 15, S135-S158. 
Berntsen, D., \& Rubin, D. C. (2006). The centrality of event scale: A measure of integrating a trauma into one's identity and its relation to post-traumatic stress disorder symptoms. Behaviour Research and Therapy, 44, 219-231.

Berntsen, D., \& Rubin, D. C. (2007). When a trauma becomes a key to identity: Enhanced integration of trauma memories predicts posttraumatic stress disorder symptoms. Applied Cognitive Psychology, 21, 417-431.

Berntsen, D., Willert, M., \& Rubin, D. C. (2003). Splintered memories or vivid landmarks? Qualities and organization of traumatic memories with and without PTSD. Applied Cognitive Psychology, 17, 675-693.

Bornstein, B. H., Liebel, L. M., \& Scarberry, N. C. (1998). Repeated testing in eyewitness memory: A means to improve recall of a negative emotional event. Applied Cognitive Psychology, 12, 119-131.

Bouman, T. K., Luteijn, F., Albersnagel, F. A., \& van der Ploeg, F. A. E. (1985). Enige ervaringen met de Beck Depression Inventory [Some experiences with the Beck Depression Inventory]. Gedrag - Tijdschrift voor Psychologie, 13, 13-24.

Brewin, C., Reynolds, M., \& Tata, P. (1999). Autobiographical memory processes and the course of depression. Journal of Abnormal Psychology, 108, 511-517.

Brewin, C. R. (1998). Intrusive autobiographical memories in depression and post-traumatic stress disorder. Applied Cognitive Psychology, 12, 359-370.

Brewin, C. R. (2005). Systematic review of screening instruments for adults at risk of PTSD. Journal of Traumatic Stress, 18, 53-62.

Brewin, C. R. (2007). Autobiographical memory for trauma: Update on four controversies. Memory, 15, 227-248.

Brewin, C. R., Christodoulides, J., \& Hutchinson, G. (1996). Intrusive thoughts and intrusive memories in a nonclinical sample. Cognition and Emotion, 10, 107-112.

Brewin, C. R., Dalgleish, T., \& Joseph, S. (1996). A dual representation theory of posttraumatic stress disorder. Psychological Review, 103, 670-686.

Brewin, C. R., Hunter, E., Carroll, F., \& Tata, P. (1996). Intrusive memories in depression: An index of schema activation? Psychological Medicine, 26, 1271-1276.

Brewin, C. R., Reynolds, M., \& Tata, P. (1999). Autobiographical memory processes and the course of depression. Journal of Abnormal Psychology, 108, 511-517.

Brewin, C. R., Watson, M., McCarthy, S., Hyman, P., \& Dayson, D. (1998). Memory processes and the course of anxiety and depression in cancer patients. Psychological Medicine, 28, 219224.

Brittlebank, A. D., Scott, J., Williams, J. M. G., \& Ferrier, I. N. (1993). Autobiographical memory in depression: State or trait marker? British Journal of Psychiatry, 162, 118-121.

Brom, D., \& Kleber, R. J. (1985). De Schok Verwerkings Lijst [The Impact of Event Scale]. Nederlands Tijdschrift voor de Psychologie, 40, 164-168. 
Brown, R., \& Kulik, J. (1977). Flashbulb memories. Cognition, 5, 73-99.

Buijsrogge, S. (2007). Autobiographical memory specificity, personality characteristics and susceptibility to misinformation. Unpublished Masters thesis, Maastricht University, Maastricht.

Burgess, P. W., \& Shallice, T. (1996). Confabulation and the control of recollection. Memory, 4, 359411.

Burnside, E., Startup, M., Byatt, M., Rollinson, L., \& Hill, J. (2004). The role of overgeneral autobiographical memory in the development of adult depression following childhood trauma. British Journal Clinical Psychology, 43, 365-376.

Bywaters, M., Andrade, J., \& Turpin, G. (2004). Intrusive and non-intrusive memories in a nonclinical sample: The effects of mood and affect on imagery vividness. Memory, 12, 467478.

Cahill, L., Gorski, L., \& Le, K. (2003). Enhanced human memory consolidation with post-learning stress: Interaction with the degree of arousal at encoding. Learning and Memory, 10, 270274.

Cahill, L., \& McGaugh, J. L. (1998). Mechanisms of emotional arousal and lasting declarative memory. Trends in Neuroscience, 21, 294-299.

Camp, G., Pecher, D., \& Schmidt, H. G. (in press). No retrieval-induced forgetting using itemspecific independent cues: Evidence against a general inhibition account. Journal of Experimental Psychology: Learning, Memory, and Cognition.

Candel, I., Merckelbach, H., \& Zandbergen, M. (2003). Boundary distortions for neutral and emotional pictures. Psychonomic Bulletin and Review, 10, 691-695.

Carlier, I. V., Voerman, B. E., \& Gersons, B. P. (2000). Intrusive traumatic recollections and comorbid posttraumatic stress disorder in depressed patients. Psychosomatic Medicine, 62, 26-32.

Chapman, L. J., \& Chapman, J. P. (1973). Problems in the measurement of cognitive deficit. Psychological Bulletin, 79, 380-385.

Chapman, L. J., \& Chapman, J. P. (1978). The measurement of differential deficit. Journal of Psychiatry, 14, 303-311.

Christianson, S., \& Loftus, E. F. (1991). Remembering emotional events: The fate of detailed information. Cognition and Emotion, 5, 81-108.

Christianson, S. A. (1992). Emotional stress and eyewitness memory: A critical review. Psychological Bulletin, 112, 284-309.

Christianson, S. A., \& Loftus, E. F. (1987). Memory for traumatic events. Applied Cognitive Psychology, 1, 225-239.

Chu, S., \& Downes, J. J. (2002). Proust nose best: Odors are better cues of autobiographical memory. Memory and Cognition, 30, 511-518. 
Ciranni, M. A., \& Shimamura, A. P. (1999). Retrieval-induced forgetting in episodic memory. Journal of Experimental Psychology: Learning, Memory, and Cognition, 25, 1403-1414.

Coffey, S. F., Gudmundsdottir, B., Beck, G., Palyo, S. A., \& Miller, L. (2006). Screening for PTSD in motor vehicle accident survivors using the PSS-SR and IES. Journal of Traumatic Stress, 19, 119-128.

Conway, M. A. (2005). Memory and the self. Journal of Memory and Language, 53, 594-628.

Conway, M. A., Anderson, S. J., Larsen, S. F., Donnelly, C. M., McDaniel, M. A., McClelland, A. G. R., et al. (1994). The formation of flashbulb memories. Memory and Cognition, 22, 326343.

Conway, M. A., \& Pleydell-Pearce, C. W. (2000). The construction of autobiographical memories in the self-memory system. Psychological Review, 107, 261-288.

Conway, M. A., Singer, J. A., \& Tagini, A. (2004). The self and autobiographical memory: Correspondence and coherence. Social Cognition, 22, 491-529.

Crane, C., Barnhofer, T., \& Williams, J. M. G. (2007). Cue self-relevance affects autobiographical memory specificity in individuals with a history of major depression. Memory, 15, 312-323.

Dalgleish, T. (2004). Cognitive approaches to Posttraumatic Stress Disorder (PTSD): The evolution of multi-representational theorizing. Psychological Bulletin, 130, 228-260.

Dalgleish, T., Perkins, H., Williams, J. M. G., Golden, A.-M., Barnard, P. J., Au-Yeung, C., et al. (2007). Reduced specificity of autobiographical memory and depression: The role of executive processes. Journal of Experimental Psychology: General, 136, 23-42.

Dalgleish, T., Rolfe, J., Golden, A., Dunn, B. D., \& Barnard, P. J. (in press). Reduced autobiographical memory specificity and posttraumatic stress: Exploring the contributions of impaired executive control and affect regulation. Journal of Abnormal Psychology.

Dalgleish, T., Spinks, H., Yiend, J., \& Kuyken, W. (2001). Autobiographical memory style in seasonal affective disorder and its relationship to future symptom remission. Journal of Abnormal Psychology, 110, 335-340.

Dalgleish, T., Tchanturia, K., Serpell, L., Hems, S., De Silva, P., \& Treasure, J. (2003). Self-reported parental abuse relates to autobiographical memory style in patients with eating disorders. Emotion, 3, 211-222.

Davies, M. I., \& Clark, D. M. (1998). Thought suppression produces a rebound effect with analogue post-traumatic intrusions. Behaviour Research and Therapy, 36, 571-582.

de Decker, A., Hermans, D., Raes, F., \& Eelen, P. (2003). Autobiographical memory specificity and trauma in inpatient adolescents. Journal of Clinical Child and Adolescent Psychology, 32, 22-31.

van der Does, A. J. W. (2002 ). Handleiding bij de Nederlandse versie van Beck Depression Inventory - second edition (BDI - II - NL) [Manual of the Dutch version of the BDI-II). San Antonio: TX / Amsterdam, NL: Harcourt. 
de Groot, M. H. (1991). Psychometrische aspecten van een stemmingsschaal (verkorte POMS). [Psychometric properties of a mood scale (shortened POMS)]. Gedrag en Gezondheid, 20, 46-51.

Ehlers, A., \& Clark, D. (2000). A cognitive model of posttraumatic stress disorder. Behaviour Research and Therapy, 38, 319-345.

Ehlers, A., Clark, D. M., Hackmann, A., McManus, F., \& Fennell, M. (2005). Cognitive therapy for post-traumatic stress disorder: Development and evaluation. Behaviour Research and Therapy, 43, 413-431.

Ehlers, A., Michael, T., Chen, Y. P., Payne, E., \& Shan, S. (2006). Enhanced perceptual priming for neutral stimuli in a traumatic context: A pathway to intrusive memories? Memory, 14, 316328.

Engelhard, I. M., Arntz, A., \& van den Hout, M. A. (in press). Limited specificity of PTSD symptoms: A comparison of patients with PTSD, healthy controls, and patients with other anxiety disorders. British Journal of Clinical Psychology.

Engelhard, I. M., van den Hout, M. A., \& Arntz, A. (2001). Posttraumatic stress disorder after pregnancy loss. General Hospital Psychiatry, 23, 62-66.

Engelhard, I. M., van den Hout, M. A., \& Kindt, M. (2003). The relationship between neuroticism, pre-traumatic stress, and post-traumatic stress: A prospective study. Personality and Individual Differences, 35, 381-388.

Engelhard, I. M., van Rij, M., Boullart, I., Ekhart, T. H., Spaanderman, M. E., van den Hout, M. A., et al. (2002). Posttraumatic stress disorder after pre-eclampsia: An exploratory study. General Hospital Psychiatry, 24, 260-264.

Evans, J., Williams, J. M. G., O'Loughlin, S., \& Howells, K. (1992). Autobiographical memory and problem-solving strategies of parasuicide patients. Psychological Medicine, 22, 399-405.

Finkenauer, C., Luminet, O., Gisle, L., El-Ahmadi, A., van der Linden, M., \& Philippot, P. (1998). Flashbulb memories and the underlying mechanisms of their formation: Toward an emotional-integrative model. Memory and Cognition, 26, 516-531.

Fleiss, J. L. (1986 ). The design and analysis of clinical experiments. New York: Wiley.

Foa, B. E., Riggs, S. D., Dancu, C. V., \& Rotbaum, B. O. (1993). Reliability and validity of a brief instrument for assessing post-traumatic stress disorder. Journal of Traumatic Stress, 6 , 459-473.

Foa, E. B., \& Kozak, M. J. (1986). Emotional processing of fear: Exposure to corrective information. Psychological Bulletin, 99, 20-35.

Frederick, I. O., Williams, M. A., Sales, A. E., Martin, D. P., \& Killien, M. (2007). Pre-pregnancy body mass index, gestational weight gain, and other maternal characteristics in relation to infant birth weight. Maternal and Child Health Journal, DOI: 10.1007/s10995-1000710276-10992. 
Geraerts, E., Kozaric-Kovacic, D., Merckelbach, H., Peraica, T., Jelicic, M., \& Candel, I. (2007). Traumatic memories of war veterans: Not so special after all. Consciousness and Cognition, 16, 170-177.

Geraerts, E., Merckelbach, H., Jelicic, M., \& Smeets, E. (2006). Long term consequences of suppression of intrusive anxious thoughts and repressive coping. Behaviour Research and Therapy, 44, 1451-1460.

Gibbs, B. R., \& Rude, S. S. (2004). Overgeneral autobiographical memory as depression vulnerability. Cognitive Therapy and Research, 28, 511-526.

Goddard, L., Dritschel, B., \& Burton, A. (1996). The role of autobiographical memory in social problem-solving and depression. Journal of Abnormal Psychology, 105, 609-616.

Goddard, L., Dritschel, B., \& Burton, A. (1997). Social problem solving and autobiographical memory in non-clinical depression. British Journal of Clinical Psychology, 36, 449-451.

Goddard, L., Pring, L., \& Felmingham, N. (2005). The effects of cue modality on the quality of personal memories retrieved. Memory, 13, 79-86.

Golden, A., Dalgleish, T., \& Mackintosh, B. (in press). Levels of specificity of autobiographical memories and of biographical memories of the deceased in bereaved individuals with and without complicated grief. Journal of Abnormal Psychology.

Groenestijn, M. A. C., Akkerhuis, G. W., Kupka, R. W., Schneider, N., \& Nolen, W. A. (1999). Gestructureerd klinisch interview voor de vaststelling van DSM-IV As I stoornissen. (Structured clinical interview for DSM-IV axis I disorders). Lisse: Swets \& Zeitlinger B.V.

Gudjonsson, G. H., \& Clare, I. C. H. (1995). The relationship between confabulation and intellectual ability, memory, interrogative suggestibility and acquiescence. Personality and Individual Differences, 19, 333-338.

Hackmann, A., Ehlers, A., Speckens, A., \& Clark, D. M. (2004). Characteristics and content of intrusive memories in PTSD and their changes with treatment. Journal of Traumatic Stress, 17, 231-240.

Halligan, S. L., Clark, D. M., \& Ehlers, A. (2002). Cognitive processing, memory, and the development of PTSD symptoms: Two experimental analogue studies. Journal of Behavior Therapy and Experimental Psychiatry, 33, 73-89.

Harvey, A. G., \& Bryant, R. A. (1998). The effect of attempted thought suppression in acute stress disorder. Behaviour Research and Therapy, 36, 583-590.

Harvey, A. G., Bryant, R. A., \& Dang, S. T. (1998). Autobiographical memory in acute stress disorder. Journal of Consulting and Clinical Psychology, 66, 500-506.

Hauer, B. J. A., Wessel, I., Geraerts, E., Merckelbach, H., \& Dalgleish, T. (in press). Autobiographical memory specificity after manipulating retrieval cues in adults reporting childhood sexual abuse. Journal of Abnormal Psychology.

Hauer, B. J. A., Wessel, I., \& Merckelbach, H. (2006). Intrusions, avoidance and overgeneral memory in a non-clinical sample. Clinical Psychology and Psychotherapy, 13, 264-268. 
Henderson, D., Hargreaves, I., Gregory, S., \& Williams, J. M. G. (2002). Autobiographical memory and emotion in a non-clinical sample of women with and without a reported history of childhood sexual abuse. British Journal of Clinical Psychology, 41, 129-142.

Henkel, L. A. (2004). Erroneous memories arising from repeated attempts to remember. Journal of Memory and Language, 50, 2-46.

Herlihy, J., Scragg, P., \& Turner, S. (2002). Discrepancies in autobiographical memories implications for the assessment of asylum seekers: Repeated interviews study. British Medical Journal, 324, 324-327.

Hermans, D., Defranc, A., Raes, F., Williams, J. M., \& Eelen, P. (2005). Reduced autobiographical memory specificity as an avoidant coping style. British Journal of Clinical Psychology, 44, 583-589.

Hermans, D., van den Broeck, K., Belis, G., Raes, F., Pieters, G., \& Eelen, P. (2004). Trauma and autobiographical memory specificity in depressed inpatients. Behaviour Research and Therapy, 42, 775-789.

Hertel, P. T. (2000). The cognitive-initiative account of depression-related impairments in memory. In D. Medin (Ed.), The psychology of learning and motivation. (Vol. 39, pp. 47-71). New York: Academic Press.

Hertel, P. T., \& Gerstle, M. (2003). Depressive deficits in forgetting. Psychological Science, 14, 573578.

Heuer, F., \& Reisberg, D. (1990). Vivid memories of emotional events: the accuracy of remembered minutiae. Memory and Cognition, 18, 496-506.

Holmes, E. A., Brewin, C. R., \& Hennesy, R. G. (2004). Trauma films, information processing, and intrusive memory development. Journal of Experimental Psychology: General, 133, 3-22.

Holmes, E. A., Brown, R. J., Mansell, W., Fearon, R. P., Hunter, E. C. M., Frasquilho, F., et al. (2005). Are there two qualitatively distinct forms of dissociation? A review and some clinical implications. Clinical Psychology Review, 25, 1-23.

Horowitz, M. J., Wilner, N., \& Alvarez, W. (1979). Impact of Event scale: A measure of subjective stress. Psychosomatic Medicine, 41, 209-218.

Janoff-Bulman, R. (1992). Shattered assumptions: Towards a new psychology of trauma. New York: Free Press.

Jones, B., Heard, H., Startup, M., Swales, M., Williams, J. M. G., \& Jones, R. S. P. (1999). Autobiographical memory and dissociation in borderline personality disorder. Psychological Medicine, 29, 1397-1404.

Kaney, S., Bowen, J. K., \& Bentall, R. P. (1999). Persecutory delusions and autobiographical memory. British Journal of Clinical Psychology, 38, 97-102.

Kangas, M., Henry, J. L., \& Bryant, R. A. (2005). A prospective study of autobiographical memory and posttraumatic stress disorder following cancer. Journal of Consulting and Clinical Psychology, 73, 293-299. 
Kelly, A. E., \& Kahn, J. H. (1994). Effects of suppression of personal intrusive thoughts. Journal of Personality and Social Psychology, 66, 998-1006.

Kremers, I. P., Spinhoven, P., \& van der Does, A. J. W. (2004). Autobiographical memory in depressed and non-depressed patients with borderline personality disorder. British Journal of Clinical Psychology, 43, 17-29.

Kuyken, W., \& Brewin, C. R. (1995). Autobiographical memory functioning in depression and reports of early abuse. Journal of Abnormal Psychology, 104, 585-591.

Kuyken, W., \& Dalgleish, T. (1995). Autobiographical memory and depression. British Journal of Clinical Psychology, 33, 89-92.

Kuyken, W., Howell, R., \& Dalgleish, T. (2006). Overgeneral autobiographical memory in depressed adolescents with, versus without, a reported history of trauma. Journal of Abnormal Psychology, 115, 387-396.

Lang, P. J., Bradley, M. M., \& Cuthbert, B. N. (1998). International affective picture system (IAPS): Technical manual and affective ratings. Centre for research in psychophysiology, University of Florida: Gainesville.

Levy, B. J., \& Anderson, M. C. (2002). Inhibitory processes and the control of memory retrieval. Trends in Cognitive Sciences, 6, 229-305.

Loftus, E. F. (2005). Planting misinformation in the human mind: A 30-year investigation of the malleability of memory. Learning and Memory, 12, 361-366.

Mackinger, H. F., Loschin, G. G., \& Leibetseder, M. M. (2000). Prediction of postnatal affective changes by autobiographical memories. European Psychologist, 5, 52-61.

Mackinger, H. F., Pachinger, M. M., Leibetseder, M. M., \& Fartacek, R. R. (2000). Autobiographical memories in women remitted from major depression. Journal of Abnormal Psychology, 109, 331-334.

MacLeod, C. M., Dodd, M. D., Sheard, E. D., Wilson, D. E., \& Bibi, U. (2003). In opposition to inhibition. In B. R. Ross (Ed.), The psychology of learning and motivation: Advances in research and theory (Vol. 43, pp. 163-214). New York, NY, US: Elsevier Science.

MacLeod, M. D. (2002). Retrieval-induced forgetting in eyewitness memory: Forgetting as a consequence of remembering. Applied Cognitive Psychology, 16, 135-149.

Macrae, C. N., \& MacLeod, M. D. (1999). On recollections lost: When practice makes imperfect. Journal of Personality and Social Psychology, 77, 463-473.

Macrae, C. N., \& Rosevaere, T. A. (2002). I was always on my mind: The self and temporary forgetting. Psychonomic Bulletin and Review, 9, 611-614.

McNair, D. M., Lorr, M., \& Droppleman, L. F. (1992). Profile of Mood States (POMS) Manual. San Diego, CA: EdITS.

McNally, R. J. (2003). Remembering trauma. Cambridge: Belknap Press of Harvard University Press. 
McNally, R. J., Clancy, S. A., Barrett, H. M., \& Parker, H. A. (2004). Inhibiting retrieval of trauma cues in adults reporting histories of childhood sexual abuse. Cognition and Emotion, 18, 479-493.

McNally, R. J., Clancy, S. A., Barrett, H. M., \& Parker, H. A. (2005). Reality monitoring in adults reporting repressed, recovered, or continuous memories of childhood sexual abuse. Journal of Abnormal Psychology, 114, 147-152.

McNally, R. J., Clancy, S. A., Barrett, H. M., Parker, H. A., Ristuccia, C. S., \& Perlman, C. A. (2006). Autobiographical memory specificity in adults reporting repressed, recovered, or continuous memories of childhood sexual abuse. Cognition and Emotion, 3/4, 527-535.

McNally, R. J., Lasko, N. B., Macklin, M. L., \& Pitman, R. K. (1995). Autobiographical memory disturbance in combat-related post-traumatic stress disorder. Behaviour Research and Therapy, 33, 619-630.

McNally, R. J., Litz, B. T., Prassas, A., Shin, L. M. F., \& Weathers, F. W. (1994). Emotional priming of autobiographical memory in post-traumatic stress disorder. Cognition and Emotion, 8, 351-367.

McNally, R. J., Litz, B. T., Prassas, A., Shin, L. N., \& Weathers, F. (1994). Emotional priming and autobiographical memory in posttraumatic stress disorder. Cognition and Emotion, 8, 351368.

Meesters, C., Merckelbach, H. L. G. J., Muris, P., \& Wessel, I. (2000). Autobiographical memory and trauma in adolescents. Journal of Behavior Therapy and Experimental Psychiatry, 31, 29-39.

Merckelbach, H., Dekkers, T., Wessel, I., \& Roefs, A. (2003a). Amnesia, flashbacks, nightmares, and dissociation in aging concentration camp survivors. Behaviour Research and Therapy, 41, 351-360.

Merckelbach, H., Dekkers, T., Wessel, I., \& Roefs, A. (2003b). Dissociative symptoms and amnesia in Dutch concentration camp survivors. Comprehensive Psychiatry, 44, 65-69.

Merckelbach, H., Muris, P., \& Horselenberg, R. (1996). Correlates of overgeneral memories in normal subjects. Behavioural and Cognitive Therapy, 24, 109-115.

Merckelbach, H., Zeles, G., van Bergen, S., \& Giesbrecht, T. (2007). Trait dissociation and commission errors in memory reports of emotional events. American Journal of Psychology, 120, 1-14.

van Minnen, A., Wessel, I., Verhaak, C., \& Smeenk, J. (2005). The relationship between autobiographical memory specificity and depressed mood following a stressful life event: A prospective study. British Journal of Clinical Psychology, 44, 405-415.

Moore, S. A., \& Zoellner, L. A. (2007). Overgeneral autobiographical memory and traumatic events: An evaluative review. Psychological Bulletin, 133, 419-437.

Moors, A., \& de Houwer, J. (2006). Automaticity: A theoretical and conceptual analysis. Psychological Bulletin, 132, 297-326. 
Morgan, H. J., \& Janoff-Bulman, R. (1994). Positive and negative self-complexity: Patterns of adjustment following traumatic versus non-traumatic life experiences. Journal of Social and Clinical Psychology, 13, 63-85.

Neal, L. A., Busuttil, W., Rollins, J., Herepath, R., Strike, P., \& Turnbull, G. (1994). Convergent validity of measures of posttraumatic stress disorder in a mixed military and civilian population. Journal of Traumatic Stress, 7, 447-455.

Nelson, K., \& Fivush, R. (2004). The emergence of autobiographical memory: A social cultural developmental theory. Psychological Review, 111, 486-511.

Nolen-Hoeksema, S. (2000). The role of rumination in depressive and mixed anxiety/depressive symptoms. Journal of Abnormal Psychology, 109, 504-511.

Olde, E., van der Hart, O., Kleber, R., \& van Son, M. (2006). Posttraumatic stress following childbirth: A review. Clinical psychology Review, 26, 1-16.

Orbach, Y., Lamb, M. E., Sternberg, K. J., Williams, J. M. G., \& Dawud-Noursi, S. (2001). The effect of being a victim or a witness of family violence on the retrieval of autobiographical memories. Child Abuse and Neglect, 25, 1427-1437.

Peeters, F. P. M. L., Wessel, I., Merckelbach, H., \& Boon-Vermeeren, B. (2002). Autobiographical memory and the course of depressive disorder. Comprehensive Psychiatry, 43, 344-350.

Pennebaker, J. (Ed.). (1995). Emotion, disclosure and health. Washington DC: American Psychological Association.

Perfect, T., Stark, L.-J., Tree, J. J., Moulin, C. J. A., Ahmed, L., \& Hutter, R. (2004). Transfer appropriate forgetting: The cue-dependent nature of retrieval-induced forgetting. Journal of Memory and Language, 51, 399-417.

Philippot, P., Schaefer, A., \& Herbette, G. (2003). Consequences of specific processing of emotional information: Impact of general versus specific autobiographical memory priming on emotion elicitation. Emotion, 270-283.

Pillemer, D. B. (1998). Momentous events, vivid memories. Cambridge, MA: Harvard University Press.

Porter, S., \& Birt, A. R. (2001). Is traumatic memory special? A comparison of traumatic memory characteristics for other emotional life events. Applied Cognitive Psychology, 15, S101S117.

Quinn, K. A., Hugenberg, K., \& Bodenhausen, G. V. (2004). Functional modularity in stereotype representation. Journal of Experimental Social Psychology, 40, 519-527.

Racsmány, M., \& Conway, M. A. (2006). Episodic inhibition. Journal of Experimental Psychology: Learning, Memory, and Cognition, 32, 44-57.

Raes, F., Hermans, D., de Decker, A., Williams, J. M. G., \& Eelen, P. (2003). Autobiographical memory specificity and affect regulation: An experimental approach. Emotion, 3, 201-206. 
Raes, F., Hermans, D., Williams, J. M., Beyers, W., Brunfaut, E., \& Eelen, P. (2006). Reduced autobiographical memory specificity and rumination in predicting the course of depression. Journal of Abnormal Psychology, 115, 699-704.

Raes, F., Hermans, D., Williams, J. M., Demyttenaere, K., Sabbe, B., Pieters, G., et al. (2005). Reduced specificity of autobiographical memory: A mediator between rumination and ineffective social problem-solving in major depression? Journal of Affective Disorders, 87, 331-335.

Raes, F., Hermans, D., Williams, J. M., \& Eelen, P. (2005). Autobiographical memory specificity and emotional abuse. British Journal of Clinical Psychology, 44, 133-138.

Raes, F., Hermans, D., Williams, J. M. G., Brunfaut, E., Hamelinck, L., \& Eelen, P. (2006). Reduced autobiographical memory specificity and trauma in major depression: On the importance of post-trauma coping versus mere trauma exposure. In F. Columbus (Ed.), Child abuse and its impact. New York: Nova Science Publishers, Inc.

Raes, F., Hermans, D., Williams, J. M. G., Demyttenaere, K., Sabbe, B., Pieters, G., et al. (2006). Is overgeneral autobiographical memory an isolated memory phenomenon in major depression? Memory, 14, 584-594.

Raes, F., Hermans, D., Williams, J. M. G., \& Eelen, P. (2006). Reduced autobiographical memory specificity and affect regulation. Cognition and Emotion, 3/4, 402-429.

Ramponi, C., Barnard, P., \& Nimmo-Smith, I. (2004). Recollection deficits in dysphoric mood: An effect of schematic models and executive mode? Memory, 12, 655-670.

Raven, J. (2000). The Ravens' progressive matrices: Change and stability over culture and time. Cognitive Psychology, 41, 1-48.

Reisberg, D., \& Heuer, F. (2004). Memory for emotional events. In D. Reisberg \& P. T. Hertel (Eds.), Memory and Emotion (pp. 3-41). Oxford, U.K.: Oxford University Press.

Reynolds, M., \& Brewin, C. R. (1999). Intrusive memories in depression and posttraumatic stress disorder. Behaviour Research and Therapy, 37, 201-215.

Riutort, M., Cuervo, C., Danion, J. M., Peretti, C. S., \& Salame, P. (2003). Reduced levels of specific autobiographical memories in schizophrenia. Psychiatry Research, 117, 35-45.

Roediger, H. L., Jacoby, J. D., \& McDermott, K. B. (1996). Misinformation effects in recall: Creating false memories through repeated retrieval. Journal of Memory and Language, 35, 300318.

Roediger, H. L., \& McDermott, K. B. (1995). Creating false memories: Remembering words not presented in lists. Journal of Experimental Psychology: Learning Memory and Cognition, 21, 803-814.

Rubin, D. C. (1996). Remembering our past: Studies in autobiographical memory. New York: Cambridge University Press.

Rubin, D. C., \& Kozin, M. (1984). Vivid memories. Cognition, 16, 81-95. 
Saunders, J., \& MacLeod, M. D. (2002). New evidence on the suggestibility of memory: The role of retrieval-induced forgetting in misinformation effects. Journal of Experimental Psychology: Applied, 8, 127-142.

Saunders, J., \& MacLeod, M. D. (2006). Can inhibition resolve retrieval competition through the control of spreading activation? Memory and Cognition, 34, 307-322.

Scher, C. D., Ingram, R. E., \& Segal, Z. V. (2005). Cognitive reactivity and vulnerability: Empirical evaluation of construct activation and cognitive diatheses in unipolar depression. Clinical Psychology Review, 25, 487-510.

Schönfeld, S., \& Ehlers, A. (2006). Overgeneral memory extends to pictorial retrieval cues and correlates with cognitive features in posttraumatic stress disorder. Emotion, 6, 611-621.

Schönfeld, S., Ehlers, A., Böllinghaus, I., \& Rief, W. (2007). Overgeneral memory and suppression of trauma memories in posttraumatic stress disorder. Memory, 15, 339-352.

Schwartz, B. L., Fisher, R. P., \& Hebert, K. S. (1998). The relation of output order and commission errors in free recall and eyewitness accounts. Memory, 6, 257-275.

Shalev, A. Y., Freedman, S., Peri, T., Brandes, D., Sahar, T., Orr, S. P., et al. (1998). Prospective study of posttraumatic stress disorder and depression following trauma. American Journal of Psychiatry, 155, 630-637.

Shaw III, J. S., Bjork, R. A., \& Handal, A. (1995). Retrieval-induced forgetting in an eyewitnessmemory paradigm. Psychonomic Bulletin and Review, 2, 249-253.

Shipherd, J. C., \& Beck, J. G. (2005). The role of thought suppression in posttraumatic stress disorder. Behavior Therapy, 36, 277-287.

Shobe, K. K., \& Klihlstrom, J. F. (1997). Is traumatic memory special? Current Directions in Psychological Science, 6, 70-74.

Smeets, T., Merckelbach, H., Horselenberg, R., \& Jelicic, M. (2005). Trying to recollect past events: Confidence, beliefs, and memories. Clinical psychology Review, 25, 917-934.

Speckens, A. E. M., Ehlers, A., Hackmann, A., Ruths, F. A., \& Clark, D. M. (2007). Intrusive memories and rumination in patients with post-traumatic stress disorder: A phenomenological comparison. Memory, 15, 249-257.

Spinhoven, P., Bockting, C. L. H., Schene, A. H., Koeter, M. W. J., Wekking, E. M., \& Williams, J. M. G. (2006). Autobiographical memory in the euthymic phase of recurrent depression. Journal of Abnormal Psychology, 115, 590-600.

Steer, P. (2005). The epidemiology of preterm labour. BJOG: An International Journal of Obstetrics and Gynaecology, 112, 1-3.

Stokes, D. J., Dritschel, B. H., \& Bekerian, D. A. (2004). The effect of burn injury on adolescents autobiographical memory. Behaviour Research and Therapy, 42, 1357-1365.

Storm, B. C., Bjork, E. L., Bjork, R. A., \& Nestojko, J. F. (2006). Is retrieval success a necessary condition for retrieval-induced forgetting? Psychonomic Bulletin and Review, 13, 10321027. 
Stuart, A. D. P., Holmes, E. A., \& Brewin, C. R. (2006). The influence of a visuospatial grounding task on intrusive images of a traumatic film. Behaviour Research and Therapy, 44, 611619.

Tabachnick, B. G., \& Fidell, L. S. (2007). Using multivariate statistics. (5th ed.). Boston, MA: Allyn \& Bacon/Pearson Education.

Tulving, E. (2002). Episodic memory and common sense: How far apart? In A. Baddeley, J. P. Aggleton \& M. A. Conway (Eds.), Episodic memory: New directions in research. (pp. 269287). New York: Oxford University Press.

Tulving, E. (2002). Episodic memory: From mind to brain. Annual Review of Psychology, 53, 1-25.

van Vreeswijk, M. F., \& de Wilde, E. J. (2004). Autobiographical memory specificity, psychopathology, depressed mood and the use of the Autobiographical Memory Test: A meta-analysis. Behaviour Research and Therapy, 42, 731-743.

Wald, F. D. M., \& Mellenbergh, G. J. (1992). De verkorte versie van de Nederlandse vertaling van de Profile of Mood Stated (POMS). [The shortened version of the Dutch translation of the Profile of Mood States (POMS)]. Nederlands Tijdschrift voor Psychologie, 45, 86-90.

Watkins, E., \& Teasdale, J. (2001). Rumination and overgeneral memory in depression: Effects of self-focus and analytic thinking. Journal of Abnormal Psychology, 110, 353-357.

Watkins, E., \& Teasdale, J. D. (2004). Adaptive and maladaptive self-focus in depression. Journal of Affective Disorders, 82, 1-8.

Watkins, E., Teasdale, J. D., \& Williams, R. W. (2000). Decentring and distraction reduce overgeneral autobiographical memory in depression. Psychological Medicine, 30, 911920.

Wegner, D. M. (1989). White bears and other unwanted thoughts. New York: Viking.

Wegner, D. M. (1994). Ironic processes of mental control. Psychological Review, 101, 34-52.

Wegner, D. M., Schneider, D. J., Carter, S. I., \& White, L. (1987). Paradoxical effects of thought suppression. Journal of Personality and Social Psychology, 53, 5-13.

Wenzlaff, R. M., \& Wegner, D. M. (2000). Thought suppression. Annual Review of Psychology, 51, 59-91.

Wessel, I., \& Hauer, B. J. A. (2006). Retrieval-induced forgetting of autobiographical memory details. Cognition and Emotion, 3/4, 430-447.

Wessel, I., Meeren, M., Peeters, F., Arntz, A., \& Merckelbach, H. (2001). Correlates of autobiographical memory specificity: The role of depression, anxiety and childhood trauma. Behaviour Research and Therapy, 39, 409-421.

Wessel, I., Merckelbach, H., \& Dekkers, T. (2002). Autobiographical memory specificity, intrusive memory, and general memory skills in Dutch-Indonesian survivors of the World War II era. Journal of Traumatic Stress, 15, 227-234.

Wessel, I., van der Kooy, P., \& Merckelbach, H. (2000). Differential recall of central and peripheral details of emotional slides is not a stable phenomenon. Memory, 8, 95-109. 
Wilhelm, S., McNally, R. J., Baer, L., \& Florin, I. (1997). Autobiographical memory in obsessivecompulsive disorder. British Journal of Clinical Psychology, 36, 21-31.

Willebrand, M., Norlund, F., Kildal, M., Gerdin, B., Ekselius, L., \& Andersson, G. (2002). Cognitive distortions in recovered burn patients: The emotional Stroop task and autobiographical memory test. Burns, 28, 465-471.

Williams, C. C., \& Zacks, R. T. (2001). Is retrieval-induced forgetting an inhibitory process? American Journal of Psychology, 114, 329-354.

Williams, J. M., Barnhofer, T., Crane, C., Herman, D., Raes, F., Watkins, E., \& Dalgleish, T. (2007). Autobiographical memory specificity and emotional disorder. Psychological Bulletin, 133, 122-148.

Williams, J. M. G. (1996). Depression and the specificity of autobiographical memory. In D. C. Rubin (Ed.), Remembering our past: Studies in autobiographical memory (pp. 244-267). New York: Cambridge University Press.

Williams, J. M. G. (2006). Capture, rumination, functional avoidance and executive control (carfax): Three processes that underlie over-general memory. Cognition and Emotion, 3/4, 548568.

Williams, J. M. G., \& Broadbent, K. (1986). Autobiographical memory in suicide attempters. Journal of Abnormal Psychology, 95, 144-149.

Williams, J. M. G., Chan, S., Barnhofer, T., Eade, J., \& Healy, H. (2006). Retrieval of autobiographical memories: The mechanisms and consequences of truncated search. Cognition and Emotion, 3/4, 351-382.

Williams, J. M. G., \& Dritschel, B. (1991). Categoric and extended autobiographical memories. In M. Conway, H. Rubin, W. Spinnler \& W. Wagenaar (Eds.), Theoretical perspectives on autobiographical memory (pp. 391-409). Dordrecht: Kluwer Academic Publishers.

Williams, J. M. G., Ellis, N. C., Tyers, C., MacLeod, A. K., \& Rose, G. (1996). Specificity of autobiographical memory and imageability of the future. Memory and Cognition, 24, 116125.

Williams, J. M. G., Healy, H. H., \& Ellis, N. C. (1999). The effect of imageability and predictability of cues in autobiographical memory. Quarterly Journal of Experimental Psychology, 52A, 555-579.

Williams, J. M. G., \& Scott, J. (1988). Autobiographical memory in depression. Psychological Medicine, 18, 689-695.

Williams, J. M. G., Stiles, W. B., \& Shapiro, D. (1999). Cognitive mechanisms in the avoidance of painful and dangerous thoughts: Elaborating the assimmilation model. Cognitive Therapy and Research, 23, 285-306.

Williams, J. M. G., Teasdale, J. D., Segal, Z. V., \& Soulsby, J. (2000). Mindfulness-based cognitive therapy reduces overgeneral autobiographical memory in formerly depressed patients. Journal of Abnormal Psychology, 109, 150-155. 
Williams, J. M. G., Watts, F. N., MacLeod, C., \& Mathews, A. (1997). Cognitive psychology and emotional disorders (2nd ed.). Chichester: Wiley.

Wohlfarth, T. D., van den Brink, W., Winkel, F. W., \& ter Smitten, M. (2003). Screening for posttraumatic stress disorder: An evaluation of two self-report scales among crime victims. Psychological Assessment, 15, 101-109.

Wright, D. B., Loftus, E. F., \& Hall, M. (2001). Now you see it; now you don't: Inhibiting recall and recognition of scenes. Applied Cognitive Psychology, 15, 471-482

Zacks, R. T., \& Hasher, L. (1994). Directed ignoring: Inhibitory regulation of working memory. In D. Dagenbach \& T. Carr (Eds.), Inhibitory processes in attention, memory, and language (pp. 241-264). San Diego, CA: Academic Press. 



\section{Dankwoord / Acknowledgements}

Het zit erop. Mijn proefschrift is klaar en ik ben er trots op. Toen ik ruim vier jaar geleden aan dit project begon, had ik geen idee van wat er allemaal op mijn pad zou komen. Het is een avontuur geworden met verrassende wendingen, hoogtepunten en dieptepunten, mooie reizen en vriendschap. Of ik het achteraf nog een keer zou doen? Ik had het voor geen goud willen missen. Maar nu is het tijd voor iets nieuws.

Ik heb mijn proefschrift niet alleen geschreven. Daartoe hebben veel mensen met wie ik plezierig heb samengewerkt een bijdrage geleverd. Een aantal van hen wil ik graag persoonlijk bedanken.

Allereerst mijn promotor. Beste Harald, ik heb er bewondering voor hoe je ondanks je drukke agenda op het juiste moment de tijd neemt voor begeleiding. Ik wil je bedanken voor je uitermate snelle en buitengewoon grondige commentaar op de artikelen die ik je stuurde. Met je inspirerende ideeën en pragmatische kijk heb je me regelmatig met een andere invalshoek naar data laten kijken. Bedankt, ik heb daar veel van geleerd. Beste Ineke, copromotor, ik denk dat we elkaar vaker 'op locatie' hebben gezien dan in onze werkkamers in Groningen en Maastricht. Los van het buitenland (bijvoorbeeld in New Orleans, Oxford, St Andrews, of Sydney), hebben we elkaar denk ik vooral in Utrecht ontmoet. Meestal in 'Oudaen' of 'De winkel van Sinkel', waar we dan soms de hele dag experimenten, resultaten of papers bespraken. Ondertussen gingen we van de koffie naar de lunch en van een kop thee over op een borrel. Ik wil je bedanken voor je inspirerende ideeën, scherpe discussies en je grondige feedback. Ik heb daar ontzettend veel van geleerd. Bedankt ook voor je vertrouwen en je support om mijn eigen weg te volgen. Dear Tim, copromotor, it is a great pleasure to work with you. You are a very kind person. I have learned a lot from you about science, academia, and especially about writing. My stay in Cambridge was the highlight of my PhD period. I have had a lovely time, in which I had the opportunity to meet many interesting people. Thank you very much for your hospitality. And I am happy that you will come to Maastricht to attend my public defense. 
De zwangerschapsstudie was nooit van de grond gekomen zonder jou, Iris. Hartelijk bedankt voor de kans die je me hebt gegeven om een klinische studie te doen. Ook Louis Peeters wil ik bedanken voor de samenwerking en de mogelijkheid die hij heeft geboden om zijn patiënten te testen. Beste Annie, er waren weken dat we tevergeefs bijna iedere dag naar het ziekenhuis liepen om te kijken of er weer patiënten waren binnengekomen om te testen. Ik heb er plezier aan beleefd dat we dit samen konden doen. Bedankt voor het testen van de vrouwen en alle administratieve taken om de studie goed te laten verlopen.

Elke wil ik graag bedanken voor onze samenwerking in de misbruikstudie. Het was een omvangrijke klus die mooie data heeft opgeleverd. Saskia, jij bent een buitengewoon fijne collega en inmiddels ook vriendin, ontzettend bedankt voor het doornemen van mijn proefschrift op typo's en andere onregelmatigheden. Ewout, mijn kamergenoot, we hebben vier jaar samen hard gewerkt daar op die kamer. Ik wens je succes met jouw laatste loodjes! Mijn collega's van de Interfacultaire werkgroep Rechtspsychologie (IRP) wil ik graag bedanken voor de interessante bijeenkomsten en de leerzame discussies. Ik zal goede herinneringen houden aan onze Ardennen-uitstapjes, etentjes en de koffiepauzes, waarin het nuttige meestal goed met het aangename werd verenigend. Ook bedank ik mijn collega's van de onderzoeksgroep Clinical Psychological Science voor een stimulerende onderzoeksomgeving. Mijn speciale dank gaat uit naar de ruim 700 proefpersonen die hebben deelgenomen aan de verschillende experimenten en naar de studenten die mij hebben geholpen om hen te testen.

Paranimfen Anne en Marjolijn, ik vind het bijzonder fijn dat jullie tijdens de verdediging naast me staan. Lieve Anne, je was m'n begeleider toen ik in Toronto zat en daarna was ik je student assistent. Nu zijn we collega's, maar bovenal ben je een heel goede vriendin geworden. Ik kijk met ontzettend veel plezier terug op onze ontbijtjes in de Coffeelovers, samen sporten, en de heerlijke en gezellige etentjes. We wonen niet meer bij elkaar om de hoek, maar we houden het er zeker in! Lieve Marjolijn, je bent een bijzondere vriendin. Ons voornemen om elkaar weer meer te zien, is dit laatste jaar wel tot uiting gekomen! lk heb genoten van onze etentjes bij Opazzo - een traditie om voort te zetten. Ik vind het bijzonder hoe we op de juiste momenten bij elkaar weten te zijn. 
Buiten een directe bijdrage aan mijn proefschrift, hebben vrienden en familie voor de nodige ontspanning en support gezorgd. Ex-huisgenootjes Frank, Hiske en Pauline, bedankt voor de eindeloze theesessies, Coffeelovers-middagen en gezellige spelletjesavonden. We wonen niet meer allemaal even dicht bij elkaar, maar ik hoop dat we dit soort dingen zullen blijven doen. Anne, Pascal, Marielle, Jaco, Marjan, Vincent, Marjolijn, Nicolien, Martijn, Ruth, Lodewijk, Saskia, Robert, Tineke, Robbert: bedankt voor de gezellige avondjes en heerlijke etentjes. I also thank my friends from Cambridge (Andrei, Ann-Marie, Dietsje, Eneida, Judith, Lizi, Michael, Marco, and Mihai) for making it a lovely time. Andrei, dear neighbour and 'dodgy' housemate, we had a wonderful time and fun dinner parties that I will not forget. I hope to see you all again!

Ten slotte mijn familie. Lieve ouders, pap en mam, bedankt voor jullie enthousiasme en stimulans om in dit project te stappen. Bedankt voor jullie steun en de kansen die jullie me hebben gegeven om te doen wat ik graag wil doen. Ik ben blij dat jullie deze dag met mij kunnen delen. Lieve Tesselschade, mijn zus, heb je enig idee hoeveel uren wij al aan de telefoon hebben gezeten? Je bent bijzonder en ik vind het knap hoe je je studie zo goed weet te combineren met de twee meisjes. Lieve Aad en Joke, ik vind het fijn dat ik jullie heb leren kennen. Bedankt voor jullie onvoorwaardelijke support. Liefste Nils, we did it! De afgelopen jaren hebben we samen beleefd. We hebben veel afgerond en we zijn aan nieuwe dingen begonnen. Het maakt me gelukkig dat we dit samen delen. Bedankt voor wie je bent en dat je altijd naast me staat. En natuurlijk ook bedankt voor je werk aan de lay-out en de voorkant van mijn proefschrift. We hebben er iets moois van gemaakt!

Beatrijs

Amsterdam, december, 2007 



\section{Curriculum Vitae}

Beatrijs Hauer, was born on March 28, 1980 in Utrecht in The Netherlands. She attended the 'Utrechts Stedelijk Gymnasium' in Utrecht, where she finished her secondary school in 1998. Immediately thereafter, she started her studies in psychology at Maastricht University. During this period, she was actively involved in tutoring fellow students in psychology and statistics and participated in several extra-curricular research projects. In 2002, she conducted a research project under supervision of Prof. dr. C. MacLeod at the University of Toronto in Canada. In 2003, she wrote her Master thesis entitled 'Why do we forget when we remember' under supervision of Dr. I Wessel. Alongside her studies, she continued playing her violin and joined several orchestras, such as 'Het Groot Entree orkest' (concert at the Concertgebouw in Amsterdam), the University of Toronto Chamber String Orchestra (concertmaster), and ARKA symphonic orchestra (board member).

In 2003 she started her PhD research project entitled 'Autobiographical memory retrieval: Overgeneral memory and intrusions' at the department of Clinical Psychological Science at Maastricht University. She conducted part of her research in collaboration with Dr. T. Dalgleish at the Medical Research Counsil, Cognition and Brain sciences Unit in Cambridge in the United Kingdom. She has presented original research and invited papers at international meetings and has been awarded with the 'article prize EPP', a prize for research students attached to the national research school Experimental Psychopathology, for the best article in 2006. Currently, she has an appointment as a research scientist at the department of Consumer Perception and Behaviour of the Unilever Food and Health Research Institute in Vlaardingen. 



\section{Publications}

Hauer, B., Wessel, I., Geraerts, E., Merckelbach, H., \& Dalgleish, T. (in press). Autobiographical memory specificity after manipulating retrieval cues in adults reporting childhood sexual abuse. Journal of Abnormal Psychology.

Dalgleish, T., Hauer, B., \& Kuyken, W. (in press). The mental control of autobiographical recollection in the aftermath of trauma. Current Directions for Psychological Science.

Geraerts, E., Schooler, J., Merckelbach, H., Jelicic, M., Hauer, B., \& Ambadar, Z. (2007). The reality of recovered memories: Corroborating continuous and discontinuous memories of childhood sexual abuse. Psychological Science, 18, 564-568.

Hauer, B., Wessel, I., Merckelbach, H., Roefs, A., \& Dalgleish, T. (2007). Effects of repeated retrieval of central and peripheral details in complex emotional slides. Memory, 15, 435449.

Geraerts, E., Arnold, M. M., Lindsay, D. S., Merckelbach, H., Jelicic, M., \& Hauer, B. (2006). Forgetting of prior remembering in people reporting recovered memories of childhood sexual abuse. Psychological Science, 17, 1002-1008.

Hauer, B., Merckelbach, H., \& Wessel, I. (2006). Intrusions, avoidance and overgeneral memory in a non-clinical sample. Clinical Psychology and Psychotherapy, 13, 264-268.

Hauer, B., \& MacLeod, C.M. (2006). Endogenous versus endogenous attentional cuing effects on memory. Acta Psychologica, 122, 305-320.

Wessel, I., \& Hauer, B. (2006). Retrieval-induced forgetting of autobiographical memory details. Cognition and Emotion, 20, 430-447.

Hauer, B., Wessel, I., \& Merckelbach, H. (2005). Overalgemene herinneringen bij depressie en posttraumatische stress stoornis: De rol van intrusieve herinneringen. Tijdschrift voor Psychiatrie, 5, 299-308.

Merckelbach, H., Hauer, B., \& Rassin, E. (2002). Symptom validity testing of feigned dissociative amnesia: A simulation study. Psychology, Crime \& Law, 8, 311-318. 




